Schmerz 2016 · 30 (Suppl 2):S1-S80

DOI 10.1007/s00482-016-0161-2

Online publiziert: 17. Oktober 2016

○) Springer-Verlag Berlin Heidelberg 2016

CrossMark

\section{Wissenschaftlicher Trager}

Deutsche Schmerzgesellschaft e.V.

Deutsche Migräne- und Kopfschmerzgesellschaft e.V. (DMKG)

\section{In Zusammenarbeit mit}

Deutsche Interdisziplinäre Vereinigung für Schmerztherapie (DIVS)

Deutsche Gesellschaft für Palliativmedizin e. V. (DGP) Deutsche Gesellschaft für Schmerztherapie e.V. (DGS) Deutsche Gesellschaft für Psychologische Schmerztherapie und -forschung (DGPSF)

Berufsverband der Ärzte und Psychologischen Psychotherapeuten in der Schmerz- und Palliativmedizin in Deutschland e.V. (BVSD)

Interdisziplinäre Gesellschaft für orthopädische/unfallchirurgische und allgemeine Schmerztherapie e.V. (IGOST)

\section{Kongressort}

Congress Center Rosengarten

Rosengartenplatz 2

68161 Mannheim

\section{Mitglieder des Programmkomitees}

Prof. Dr. Shahnaz Christina Azad, München

Prof. Dr. Ulrike Bingel, Essen

Prof. Dr. Hans-Raimund Casser, Mainz

PD Dr. Friedrich Ebinger, Paderborn

Dr. Elena Enax-Krumova, Bochum

Prof. Dr. Dr. Stefan Evers, Coppenbrügge

PD Dr. Stefanie Förderreuther, München

PD Dr. Charly Gaul, Königstein

PD Dr. Janne Gierthmühlen, Kiel

Prof. Dr. Winfried Häuser, Saarbrücken

PD Dr. Tim Jürgens, Rostock

PD Dr. Dipl.-Psych. Regine Klinger, Hamburg

Silke Laubscher, Heidelberg

PD Dr. Walter Magerl, Mannheim

Prof. Dr. Christian Maihöfner, Fürth

Dr. Volker Malzacher, Reutlingen

Prof. Dr. Martin Marziniak, Haar

Prof. Dr. Winfried Meissner, Jena

Prof. Dr. Dr. Joachim Nadstawek, Bonn

Dr. Paul Nilges, Mainz

Prof. Dr. Dr. h.c. Jürgen Osterbrink, Salzburg (AT)

Prof. Dr. Frank Petzke, Göttingen

Prof. Dr. Michael Pfingsten, Göttingen

Prof. Dr. Esther Pogatzki-Zahn, Münster

Prof. Dr. Lukas Radbruch, Berlin

Prof. Dr. Hans-Georg Schaible, Jena

Prof. Dr. Michael Schäfer, Berlin

Prof. Dr. Martin Schmelz, Mannheim

Dagmar Seeger, Göttingen

Prof. Dr. Andreas Straube, München

Monika Thomm, Köln

Prof. Dr. Rolf-Detlef Treede, Mannheim

PD Dr. Nurcan Üceyler, Würzburg

\section{Posterkomission}

Prof. Dr. Christian Maihöfner, Fürth

Prof. Dr. A. Ewers, Salzburg (AT)

Prof. Dr. P. Kropp, Rostock

Prof. Dr. A. Leffler, Hannover

PD Dr. Walter Magerl, Mannheim

Prof. Dr. Martin Marziniak, Haar

\section{(Um)Denken erwünscht}

\section{Deutscher Schmerzkongress 2016}

\author{
Liebe Kolleginnen und Kollegen,
}

mit dem Motto "(um)denken erwünscht" möchten wir Sie ganz herzlich zum Deutschen Schmerzkongress 2016 begrüßen. „Denken ist erwünscht" - wie einfach sich das anhört und wie unbequem das an manchen Stellen doch sein kann. „Umdenken ist erwünscht" auch das hören wir in vielen Bereichen unseres Lebens immer dann, wenn eingefahrene Wege uns nicht weiterbringen. Oft ist es selber schwierig, zu erkennen, wann denken und insbesondere umdenken notwendig ist. Hinzu kommt, dass gerade umdenken anstrengend ist, Flexibilität erfordert und in vielen Momenten auch selbstkritisches Hinterfragen des eigenen Tuns notwendig macht. Und so wollen wir uns auf dem diesjährigen Deutschen Schmerzkongress besonderes um solche Aspekte der Schmerzmedizin kümmern, bei denen wir in eine Sackgasse geraten sein könnten, bei denen therapeutische Erfolge ausbleiben oder bei denen wir schlicht an die Grenzen unseres Wissens gekommen sind.

Dabei gehört oft Mut zum Umdenken. Wir kennen nur allzu gut akademische Lehrmeinungen, die niemand wagt anzuzweifeln; wir denken in eingefahrenen Bahnen und können uns nicht vorstellen, dass Dinge auch anders lauf können, oft nach dem Motto, dass nicht sein kann, was nicht sein darf. In mehreren wissenschaftlichen Symposien soll mit Mythen aufgeräumt werden, die inzwischen zu einer Last in der Schmerztherapie geworden sind wie z. B. der universelle und unkritische Einsatz der Opioide. Ebenfalls werden wir in vielen Bereichen der Schmerzmedizin Wege diskutiert, die bisher wenig bekannt oder vielleicht sogar verpönt sind. In der Eröffnungsveranstaltung und im sogenannten Präsidentensymposium wollen wir eingefahrene Pfade verlassen und die Schmerzmedizin einmal von ganz anderen Seiten beleuchten, mehr sei hier nicht verraten.

Neu ist auch die Vernetzung mit anderen Fachdisziplinen, die wir in diesem Jahr mit der Neurologie starten wollen; geplant ist, jedes Jahr ein Fach besonders einzubeziehen und an einem Tag des Kongress (Freitag) Querschnitte hierzu besonders herauszuheben. Und nicht zuletzt sei hier auf die Kongressparty am Freitagabend hingewiesen, die in diesem Jahr im Museum Weltkulturen der Reiss-Engelhorn-Museen stattfinden wird und mit einer (nächtlichen) Museumstour verknüpft werden kann. Melden Sie sich hierzu früh genug an da die Plätze begrenzt sind - es wird ein besonderes, nicht alltägliches Erlebnis werden.

Natülich finden Sie neben den Versuchen des Umdenkens auch traditionelle Angebote in der Präsentation wissenschaftlicher Erkenntnisse, in den Workshops und im Beiprogramm. Zum Umdenken gehört ja auch, dass mit Verstand neue Wege eingeschlagen werden sollen und nicht alles neu erdacht werden muss, nur weil sich manches als falsch herausgestellt hat. Nicht zuletzt gehört hierzu auch die spannende Speed Update-Sitzung, in der Ihnen die aktuellsten Erkenntnisse des letzten Jahres kompakt und praxisrelevant von Experten auf hohem Niveau präsentiert werden.

Wir möchten im Rahmen dieses Kongresses auch den wissenschaftlichen Nachwuchs mit attraktiven Veranstaltungen wie beispielsweise dem Nachwuchssymposium und einem nachfolgenden Get together sowie spannenden Postersitzungen begeistern.

Wir wünschen Ihnen allen einen anregenden Deutschen Schmerzkongress 2016, der Ihnen so viel Spaß bringen möge, wie uns die Vorbereitung gemacht hat.

Es grüßen Sie herzlich

Esther Pogatzki-Zahn

und

Stefan Evers

Kongresspräsident der DMKG 



\title{
Inhaltsverzeichnis
}

\author{
Symposien
}

Donnerstag, 20.10.2016

S11 SY01 - Körperliche Aktivität, Sport und Schmerz: Interaktion von körperlicher Aktivität und Schmerzwahrnehmung

S11 SY02 - Schmerztherapeutische Versorgung von Kindern und Jugendlichen in Deutschland

S11 SY03 - Von der Pathophysiologie zur Diagnostik: Neuroplastizität bei Kopfschmerzerkrankungen

S12 SY04 - Professionell interprofessionell handeln: Wer darf was in der Akutschmerztherapie

S12 SY05 - Schmerzen innerhalb und außerhalb der Röhre - Möglichkeiten der modernen MR-Methodik und Elektrophysiologie für Forschung und Klinik

S13 SY06 - Adipositas - Proinflammatorische Zytokine - Chronischer Schmerz Einflussmöglichkeiten über Vagus- und körperliche Aktivierung SY07 Speed Update

S13 SY08 - Elektrisch evozierte Schmerz-assoziierte Potentiale bei der Diagnostik von Neuropathien und bei der Untersuchung der konditionierten Schmerzmodulation

S14 SY09 - Die altbekannten „transient receptor potential“ (TRP)-Schmerzkanäle - noch Targets für neue Schmerzmedikamente?

S14 SY10 - Kopfschmerzen und Genetik - was wissen wir, was nutzt uns dieses Wissen und was bringt die Zukunft?

S14 SY11 - Stiefkinder der Schmerzversorgung - bisher wenig beachtete Bereiche der Akutschmerztherapie

S15 SY12 - Aktuelle Erkenntnisse zur Pathophysiologie, Diagnostik und Therapie des Gelenkschmerzes (ein Beitrag zum 2016 Global Year Against Pain in the Joints)

S15 SY13 - Der gestresste Rücken: Psychobiologische Zusammenhänge von Stress und Rückenschmerz

\section{Freitag, 21.10.2016}

SY14 - Zeit zum (Um)denken bei älteren Patienten mit Rückenschmerzen SY15 - Genom und Proteom in Schmerzpathophysiologie - Welche Möglichkeiten bieten neue Analysemethoden

S17 SY16 - Update Kopfschmerz

S17 SY17 - Hypnose in der Grundlagenforschung und in der Therapie akuter und chronischer Schmerzen

S17 SY18 - Qualitätssicherung und Zertifizierung in der Multimodalen Schmerztherapie

S18 SY19-Stratifizierung von Patienten mit Nervenschmerzen: kommt die mechanismenbasierte Therapie?

S18 SY20 - Was zählt, ist normales Leben, nicht Kosmetik: Schmerztherapeutische Langzeitverläufe!

S19 SY21 - Müssen wir bei der Zentralen Sensiblisierung umdenken?

S19 SY23 - DMKG meets HNO - relevante Schmerzsyndrome von Hals, Nase und Ohr 
SY24 - Umdenken in der Akutschmerztherapie: Mythen zerplatzen lassen ...?

S20 SY25 - Konzepte, praktische Umsetzung und Evidenzlage zur Edukation von (Kopf)Schmerzpatienten

\section{Samstag, 22.10.2017}

S21 SY26 - Schmerz, Schmerzempfindlichkeit und körpereigene Schmerzhemmung - Messung, Zusammenhänge und klinische Anwendung

S21 SY27 - Das vegetative Nervensystem und Schmerz: Mögliche Zusammenhänge und therapeutische Optionen.

SY28 - Paradigmenwechsel beim Postnukleotomie-Syndrom

SY29 - Welche Rolle spielen Entzündungsvorgänge bei unseren Schmerzpatienten?

S22 SY30 - Umdenken erwünscht - zur Rolle der Psychologie in Pathophysiologie und Therapie der Migräne

SY31 - Multikulturelle Aspekte der Schmerztherapie: (Um)Denken erwünscht!

SY32 - Das Neuropeptid CGRP als Biomarker, Schlüsselmediator und therapeutisches Target inner- und außerhalb der Migräne SY33 - „Schmerz oder kein Schmerz - das ist hier die Frage." Möglichkeiten und Grenzen der Erfassung von akuten und chronischen Schmerzen SY34 - Mechanismen der Schmerzhemmung: neue Aspekte SY35 - Umdenken nötig: der schwierige Tumorschmerzpatient als Herausforderung in der Schmerzmedizin SY36 - Emotion, Motivation und Schmerz - eine (un)heilvolle Allianz? SY37 - Pathophysiologie, Diagnostik und Therapie chronischer Unterbauchschmerzen der Frau - eine interdisziplinäre Perspektive SY38 - Physiotherapie bei Migräne und Kopfschmerzen - Grundlagenforschung, Untersuchungsergebnisse und Behandlungsmöglichkeiten SY39 - Schmerzmanagement auf Intensivstationen nach Operationen Möglichkeiten und Grenzen SY40 - Hyperalgesie und Allodynie: Von der Pathophysiologie zur klinischen Relevanz

\section{Workshops}

\section{Donnerstag, 20.10.2016}

WS01 - „Ich bilde mir den Schmerz doch nicht ein" - Bio-psycho-soziale Zusammenhänge von Schmerz erklären - aber wie? WSO2 - Opioide in der Praxis WS03 - Biofeedbacktherapie bei Kopfschmerzen und Migräne WS04 - Der schwierige Fall - Ein Videoseminar zum praktischen Umgang mit Schmerzpatienten

S29 WS05 - Sonographie - Risikoreduktion für Patient und Therapeut durch sonographisch-gestützte Interventionen: gibt es wirbelsäulennah eine echte Rationale?

S29 WS06 - Akuter Schmerz-Hypnose; chronischer Schmerz-Hypnotherapie - Unterschiede und Ähnlichkeiten in der Behandlung-

S29 WS07 - Die multimodale Diagnostik und Therapie des Chronischen Rückenschmerzes

S30 WS08 - Training interpersoneller Verhaltens- und Steuerungskompetenz CBASP (Cognitive Behavioral Analysis System of Psychotherapy) für Patienten mit chronischem Schmerz

S31 WS09-Schmerzdiagnostik mit Skalen und Fragebögen 
S31 WS10 - Neurologischer Untersuchungskurs für Schmerztherapeuten: Praktische Übungen, Tricks und Syndromlehre

S31 WS12 - Umlenken erwünscht:Typische Fehldiagnosen und Möglichkeiten der interdisziplinären Aufklärung

\section{Freitag, 21.10.2016}

S31 WS13 - Achtsamkeit und Akzeptanz in der Schmerztherapie

S32 WS14 - Neuroorthopädische und funktionelle Untersuchung für Schmerzmediziner

S32 WS15 - Naturheilkunde in der Schmerztherapie

S32 WS16 - Strategien in der postoperativen Schmerztherapie anhand von Fallbeispielen

S32 WS17 - Mindfulness-Based Stress Reduction (MBSR nach Kabat-Zinn) in der Therapie chronischer Schmerzen - eine Einführung mit praktischen Übungen

WS18 - Klinische Differenzierung und gezielte Behandlung des SchulterNacken-Schmerzes Kopfschmerzversorgung in Deutschland - Quo vadis?

S34 WS22 - Stress \& Schmerz: Untersuchung und Biofeedback bei Kopf- und Gesichtsschmerz

S34 WS23 - „Wenn der Kopf zerbricht" - Hypnose und Selbsthypnose bei Kopfschmerzen

S35 WS24 - Screening in der Physiotherapie

S35 WS25 - Diagnostische und therapeutische Lokalanästhesie myofaszialer Triggerpunkte

\section{Samstag, 22.10.2016}

S35 WS26 - Behandlung schmerzbezogener Angst bei Rücken- und Kopfschmerzen

WS27 - Akupunktur für "Ahnungslose"

WS28 - Interprofessionelle Begutachtung schmerzbedingter Funktionsund Leistungseinschränkungen

S37 WS29 - Fahrtauglichkeit/Arbeitsfähigkeit unter Medikamenten - Aktueller Stand

S37 WS30 - Chronische Schmerzen bewältigen oder akzeptieren?

S37 WS31 - Studien lesen und wirklich verstehen

S38 WS32 - Sinnvolle Diagnostik und Therapie bei neuropathischen Schmerzen - ein Fallseminar

S38 WS33 - Biofeedback-Therapie bei chronischen Schmerzen

\section{Poster}

\section{P01 - Akutschmerz/Schmerz nach Operationen}

S38 P01.01 - Schmerzen in der Notfallaufnahme eines Krankenhauses - Inzidenz, Intensität und Lokalisation

S39 P01.02 - Regionalanästhesie der oberen Extremität bei Handphlegmone

S39 P01.03 - Validierung eines Risikoscores zur Vorhersage ausgeprägter postoperativer Schmerzen

S39 P01.04-Schmerzbewältigungsstrategien und postoperative Outcomes nach Hysterektomie 
S40 P01.05 - Beeinflusst das Geschlecht des Untersuchers die Bewertung akuter postoperativer Schmerzen bei Männern und Frauen? Eine prospektive Studie an Patienten nach Sternotomie.

S40 P01.06 - Wie kann die Qualität der postoperativen Analgesie bei Kindern verbessert werden?

P02 - Nicht-pharmakologische Therapieverfahren/ Pharmakologische Therapie des Schmerzes

S41 P02.01 - Einfluss Transkutaner Elektrischer Nervenstimulation (TENS) Auf Parameter Der Quantitativ Sensorischen Testung (QST)

S41 P02.02 - Die Wirkung von transkutaner Vagusnervstimulation (TVNS) auf die Wahrnehmung von wiederholten schmerzhaften Hitze-Reizen - eine randomisierte kontrollierte Crossover-Studie

S41 P02.03 - Feedbacktraining des nozizeptiven Rückziehreflexes bei chronischen Rückenschmerzpatienten: Einfluss auf klinischen Schmerz und Conditioned Pain Modulation

S42 P02.04 - Visuelles Feedback verbessert den Effekt klassischer Massagetherapie bei Patienten mit chronischen Rückenschmerzen

S42 P02.05 - Sensorische Mapping der durch die okzipitale Nervenstimulation (ONS) induzierten räumlichen, kognitiven und affektiven Empfindungen in der Behandlung der chronischen Migräne

\section{P03 - Experimentelle Schmerzmodelle (Mensch)}

S43 P03.01 - Die neuronalen Korrelate der Störfunktion von Schmerz an Gesicht und Hand und der Einfluss schmerzbezogener Angst

S43 P03.02 - Wahrnehmung von wiederholten schmerzhaften Hitze-Reizen durch gesunde Probanden - Vergleich von vier Kombinationen verschiedener Stimulationsparameter

S43 P03.03 - Antizipation vs. Realisierung eines 2-h-Ausdauerlaufes: Was wirkt schmerzhemmender?

S44 P03.04. - Periphere und zentrale Komponenten der veränderten Hitzeschmerzwahrnehmung während repetitiver Laserreizung bei gesunden Probanden

S44 P03.05 - Einfluss einer Habituation auf Schmerzintensität und schmerzevozierte Potentiale (PREP) im Vergleich zu CPM-Effekten

S44 P03.06 - Einfluss von endogenem Testosteron auf sensorische und schmerzhafte Empfindungen, sowie die körpereigene Schmerzhemmung bei Männern

S45 P03.07 - Bedeutung der körpereigenen Schmerzhemmung für Hyperalgesie nach einer Verbrennung 1. Grades bei gesunden Probanden

S45 P03.08 - Endogene Schmerzhemmung durch Schmerzlinderung als Belohnung

S46 P03.09 - Die Rolle des Kleinhirns bei Akquisition, Extinktion und Reinstatement Schmerz-assoziierter Furcht - Eine fMRT-Untersuchung im viszeralen Schmerzmodell

\section{P04 - Experimentelle Schmerzmodelle (Tiermodelle)}

S46 P04.01 - Untersuchung Bewegungs-evozierten Schmerzverhaltens bei Ratten nach einer Inzision

S46 P04.02 - Die Rolle des GABA-Transporters GAT-1 und der Glutamatdecarboxylase GAD 65 und 67 für Hyperalgesie nach Inzisionsverletzung der Ratte

S47 P04.03 - Einfluss niedrigdosierter postnataler Bestrahlung auf die zentralnervöse Prozessierung nozizeptiver Hitzereize bei Mäusen 
S47 P04.04-BOLD-Signal-basierte graphtheoretische Untersuchung der differentiellen Effekte verschiedener Anästhetika und Stimulationsparadigmen auf das laterale und mediale Schmerzsystem in Ratten

S48 P04.05 - Zerebrales Korrelat der spinalen pharmakologischen Modulation des GABAA Rezeptors in einem Tiermodell für postoperative Schmerzen

S48 P04.06 - Geschlechtsspezifische Unterschiede beim Schmerz in Osteoarthrose-Mäusen

S49 P04.07 - Zentralnervöse Effekte der Ablation von TRPV1-exprimierenden nozizeptiven Neuronen via Resiniferatoxin auf die Temperaturprozessierung

S49 P04.08 - Molekulare und funktionelle Veränderungen im nozizeptiven System der Ratte beim Diabetes mellitus - Beteiligung von TRP-Kanälen?

S49 P04.09-Immunohistochemical study of spinal glial cell activation in an animal model of non-specific low back pain

\section{P05 - Kopfschmerz/Pflege/Aus- und Weiterbildung}

S50 P05.01 - Repetitive Magnetstimulation der Nackenmuskulatur (m. trapezius) bei Patienten mit Migräne

S50 P05.02 - DreKiP - ein ambulantes multimodales Therapieprogramm für Kopfschmerzkinder

S50 P05.03 - Gesteigerte Schmerzempfindung am Kopf vor der Kopfschmerzattacke bei Migränepatienten: eine Möglichkeit zur zeitlichen Prognose von Migräneattacken?

S51 P05.04 - Die Medikamentenpause in der Therapie des Medikamentenübergebrauch-Kopfschmerzes (MÜK):Verlauf und intervenierende Variablen

S51 P05.05 - Schmerztherapie bei geriatrischen Patienten - Die Perspektive der Schmerzschwester

S52 P05.06 -Momentaner Inhalt und Umfang des Unterrichts zum Thema Chronischer Schmerz an deutschen Physiotherapieschulen sowie in Bachelor- und Masterstudiengängen.

\section{P06 - Multimodale Therapieverfahren}

S52 P06.01 - Untersuchung einer neuen, praktisch umsetzbaren und synergistischen multimodalen Intervention für die Behandlung chronischer Rückenschmerzen: Eine randomisiert-kontrollierte Multicenter Studie im Rahmen des MiSpEx-Netzwerks

S52 P06.02 - Faktorenstruktur der DASS-21 bei chronischen Schmerzpatienten

S53 P06.03 - Selbsteinschätzung des Einflusses auf den Schmerz nach einer multimodalen Schmerztherapie. 1-Jahres-Verlausbeobachtung.

S53 P06.04 - Vorläufige Ergebnisse einer randomisiert-kontrollierten Studie zur Wirksamkeit der Schmerzprovokation bei Jugendlichen mit chronischen Schmerzen

S53 P06.05 -Qigong bei chronischen Schmerzen

S54 P06.06 - Sensitivität und Spezifität von Screeningfragebogen für Depressivität in der interdisziplinären multimodalen Schmerztherapie

S54 P06.07-Zusammenhang zwischen Einschätzung des Therapieerfolges und Veränderungen in Bezug auf psychische Variablen nach interdisziplinärer multimodaler Schmerztherapie (IMST)

S54 P06.08- "Conditioned pain modulation“ als prognostischer Parameter für die Effektivität der multimodalen Schmerztherapie. 
P06.09 - Vom Chaos zum Licht - Ergebnisse einer qualitativen Einzelfallstudie zur kunst- und psychotherapeutischen Zusammenarbeit in der Multimodalen Schmerztherapie

\section{P07 - (Um)denken erwünscht}

S55 P07.01 - Schmerzintensität in der interdisziplinären multimodalen Schmerztherapie (IMST)- erste Ergebnisse einer quantitativen Untersuchung zu psychometrischen Eigenschaften (VAPAIN)

S56 P07.02 - Basisprogramm Ernährung und Bewegung Grundlegendes und Nachhaltiges zum täglichen Umgang mit chronischem Schmerz

S56 P07.03 - Fehldiagnose CRPS - L-Dopa-responsive schmerzhafte Dystonie nach Sprunggelenksfraktur

S56 P07.04 - Existieren geografische Unterschiede für die Sensitivität bei der Untersuchung thermischer Schwellen zwischen einer arktischen und einer mitteleuropäischen Population?

S57 P07.05 - Sind wir zu spät? - Erfahrungen mit dem stationären interdisziplinären Schmerzassessment

S57 P07.06 - Schmerzassessment in der vollstationären Altenhilfe - S3 Leitlinie

S58 P07.07 - Ein Kunstprojekt über den chronischen Schmerz: Eine qualitative Studie der Erfahrungen von einem Leben mit Schmerz im Zusammenhang mit der Familie von erwachsenen Patienten

\section{P08 - Neuropathischer Schmerz II}

S58 P08.01 - Die Dynamik des somatosensorischen Systems bei Patienten mit postherpetischer Neuralgie

S59 P08.02 - Capsaicin 8\% cutanes Pflaster zur Behandlung von Phantomund Stumpfschmerzen - Auswertung einer retrospektiven Fallserie nach Majoramputation

S59 P08.03 - Topisches Ambroxol zur Therapie des CRPS - Eine neue Option?

S59 P08.04 - Nachweis der positiven Wirkung von nicht-visuellen Effekten einer Lichtexposition auf Somatosensorik und Nozizeption bei CRPS

S60 P08.05 - Reliability of the English version of the painDETECT questionnaire and its pain descriptors

S60 P08.06 - Belastung durch lokalisierte, chronische postoperative neuropathische Schmerzen: Epidemiologische Daten aus einer laufenden multizentrischen Studie

S60 P08.07 - Contralaterally different sensory profiles in patients with distal symmetrical painful polyneuropathy by quantitative sensory testing (QST)

S61 P08.08-1187 Patienten mit Läsionen des Nervensystems mit und ohne paradoxe Hitzeempfindungen im somatosensorisches Profil

S61 P08.09 - Insuffiziente Regeneration der mechanischen Nervenfunktion nach peripheren Nervenverletzungen

S62 P08.10 - Calciumtoxizität in der Genese der Paclitaxel-induzierten Neuropathie

S62 P09.01 - Two loss-of-function mutations of Nav1.8 are linked to gain-ofpain in human patients

S62 P09.02 - Untersuchung der Kleinfasermorphologie beim FibromyalgieSyndrom

S63 P09.03 - Somatosensory profiles differ between patients with non-specific neck arm pain with and without heightened nerve mechanosensitivity

S63 P09.04 - Schmerz-evozierte Potentiale bei unilateralen peripheren Nervenschäden der oberen Extremität 
S64 P09.05 - Einfluss von Oxaliplatin auf spannungsabhängige Calciumkanäle in DRG-Neuronen der Ratte

S64 P09.06 - Neurotoxische und neuroprotektive Mechanismen Cisplatininduzierter schmerzhafter Polyneuropathien

S65 P09.07 - Detektion veränderter zentraler Schmerzverarbeitung bei Radikulopathiepatienten durch reduzierte Laser evozierte Potential Habituation

S65 P09.08 - Validierung eines neuen Thermotestgeräts bei Gesunden und Patienten mit Diabetes mellitus

S66 P09.09 - Contact heat evoked potentials: segmental acquisition from the lower extremities

S66 P09.10 - Eine erhöhte Schmerzintensität ist assoziiert mit geringerer Herzfrequenzvariabilität in der frühen Phase des Komplexen Regionalen Schmerzsyndroms (CRPS)

S66 P09.11 - Modulierbarkeit von Schmerz durch Small-fiber-Matrixstimulation nach Konditionierung durch Capsaicin: Identifizierung einer Sham Frequenz

\section{P10 - Psychologie und Psychotherapie des Schmerzes}

S67 P10.01 - Valenz und Arousal-Werte visueller Stimuli und ihre Rolle in der Linderung von Schmerzen: Was ist die Kraft der Bilder?

S67 P10.02 - Wirksamkeit kognitiver Verhaltenstherapie im Behandlungsschwerpunkt "Chronische Schmerzen" einer Hochschulambulanz

S67 P10.03-Kognitive Leistungsfähigkeit bei chronischen Schmerzpatienten

S68 P10.04-Moduliert psychologischer Stress Placebo- und Nocebo-Effekte auf viszeralen Schmerz und Stuhldrang?

S68 P10.05 - Einfluss von Bindungsverhalten auf chronischen und somatoformen Schmerz

S68 P10.06 - Einmalige Konditionierung macht aus neutralen Adjektiven negative Konotation

S69 P10.07 - Der Einfluss von Persönlichkeitsvariablen auf die individuelle Schmerzempfindlichkeit

\section{P11 - Rückenschmerz und Bewegungsapparat/Schmerz und Alter/Tumorschmerz und Palliativmedizin}

P11.01 - Ergebnisse eines Case-Management Projekts zur Verbesserung der Behandlung von chronischen Rückenschmerzen

S70 P11.03 - Zu alt für eine Therapie?! - Ergebnisse eines altersangepassten 3-wöchigen multimodalen Therapieprogrammes

S70 P11.04 - Schmerzassessment bei Delir: Eine Scopingreview

S71 P11.05-Schmerz-Komorbidität bei Restless-Legs-Syndrom - Argumente für eine frühzeitige Opioidtherapie?

S71 P11.06 - Mobilität und Schmerz: Gibt es Unterschiede bei Bewohnern in Altenhilfeeinrichtungen?

S72 P11.07 - Einfluss von Paracetamol, Metamizol und 4-N-Methylaminoantipyrin auf Pankreaskarzinomzellen in vitro

S72 P12.01 - Störungsspezifische Testdiagnostik auf dem Prüfstand: Vorläufige Ergebnisse eines Screening-Tools für Traumafolgestörungen bei Kindern mit stark beeinträchtigenden chronischen Schmerzen

S72 P12.02 - Wie schwer sind Deine Schmerzen? - Graduierung chronischer Schmerzen als Therapie-Outcome der interdisziplinären Schmerztherapie für Kinder und Jugendliche

S73 P12.03 - Know Body, Know Way, Know Action: Eine edukative Patientenschulung für Kinder und Jugendliche mit chronischen Schmerzen 
S73 P12.04 - Veränderung der Inanspruchnahme und Kosten nach einer intensiven pädiatrischen Schmerztherapie Ergebnisse einer GKV-Routinedatenanalyse

S73 P12.05 - Der pädiatrische Schmerzpatient: Eine Basisdatenerhebung in der Kinder- und Jugendarztpraxis

S74 P12.06 - Mit Spaß lernen. Überprüfung eines Wissensfragebogens zur animierten Psychoedukation über chronische Schmerzen bei Schulkindern

S74 P12.07 - Schmerzreduktion beim Verbandswechsel - Projekt zur Vermittlung von Bewältigungsstrategien für Kinder mit Epidermolysis bullosa 


\section{Donnerstag, 20.10.2016}

\section{Bewegungstherapie und manuelle Medizin}

\section{SY01 - Körperliche Aktivität, Sport und Schmerz: Interaktion von körperlicher Aktivität und Schmerzwahrnehmung}

\author{
J. Tesarz' , M. Hasenbring², C. Puta ${ }^{3}$, W. Eich ${ }^{4}$ \\ 'Universitätsklinik Heidelberg, Sektion Muskuloskelettaler Schmerz, \\ Heidelberg, Deutschland, ${ }^{2}$ Ruhr-Universität Bochum, Abt. für Medizinische \\ Psychologie und Medizinische Soziologie, Bochum, Deutschland, \\ ${ }^{3}$ Friedrich-Schiller-Universität Jena, Lehrstuhl für Sportmedizin, Jena, \\ Deutschland, ${ }^{4}$ Klinik für Allgemeine Innere Medizin und Psychosomatik/ \\ Uniklinik Heidelberg, Sektion Integrierte Psychosomatik (Schwerpunkt \\ Bewegungssystem), Heidelberg, Deutschland
}

Körperliche Aktivität wird in zahlreichen Leitlinien zur Behandlung chronischer Schmerzen empfohlen. Positive Effekte sind nachgewiesen, allerdings von moderater Effektstärke. Zudem berichten viele Sportler bei hohem Aktivitätsniveau über chronische Schmerzen. Inzwischen weiß man, dass körperliche Aktivität über multiple Faktoren mit dem Schmerzsystem interagiert, die zugrundeliegenden Mechanismen sind hierbei jedoch unvollständig verstanden. J. Tesarz geht zunächst anhand einer online-basierten Umfrage auf den Zusammenhang zwischen Schmerzhäufigkeit und -charakteristik bei Personen mit unterschiedlichem Aktivitätslevel ein. Ferner werden die Daten einer Metaanalyse sowie eigener Untersuchungen präsentiert, die den Zusammenhang körperlicher Aktivität mit psychophysiologischen Parametern aufzeigen und auf langfristige Veränderungen im Schmerzempfinden bei Sportlern hinweisen. Sportler verfügen dabei über eine höhere Schmerztoleranz als Kontrollpersonen, während Unterschiede in den Schmerzschwellen eher gering und heterogen sind. Darüber hinaus lassen sich Veränderungen in der endogenen Schmerzmodulation nachweisen. C. Puta erweitert die Perspektive des Vortrages von J. Tesarz um den Einfluss visueller Bewegungswahrnehmung und -imagination auf das Schmerzempfinden. Es werden experimentelle Daten präsentiert, die zeigen, dass: a) die Einschätzung sensomotorischer Merkmale visuell präsentierter Bewegungen spezifisch für die betroffene Region verändert ist; b) akuter Rückenschmerz, im Gegensatz zu chronischem Rückenschmerz oder gesunden Kontrollpersonen, mit der korrekten Einschätzung visuell präsentierter Bewegungen interferiert; c) die erwartete Intensität der Schmerzen bei vorgestellter Bewegungsausführung abhängig von der Art und Geschwindigkeit der präsentierten Bewegung ist. Die Ergebnisse werden mechanismenbasiert, modelbasiert sowie im Kontext der Empfehlungen zu körperlicher Aktivität bei Behandlung chronischer Schmerzen diskutiert. M. Hasenbring stellt Daten aus dem Forschungsverbund MiSpEx (National Research Network for Medicine in Spine Exercise) zum chronischen Rückenschmerz (CRS im Leistungssport (LS) und in der Allgemeinbevölkerung (AB) vor. Bei 267 Personen (64\% LS), die wegen CRS in physiotherapeutischer Behandlung waren, wurden Trainingsfrequenz, Schmerz, Beeinträchtigung und Schmerzverarbeitung untersucht. Erste Ergebnisse: (1) die Beziehung zwischen körperlicher Aktivität und Schmerzintensität folgte einer U-Funktion, (2) LS unterschieden sich in Schmerzintensität nicht von $\mathrm{AB}$, zeigten jedoch eine tendenziell geringere Beeinträchtigung und (3) maladaptive Muster der Schmerzwahrnehmung gingen mit höherer Schmerzintensität und Beeinträchtigung bei $\mathrm{AB}$ einher, bei LS lediglich mit Beeinträchtigung im Alltag. Die Befunde weisen auf unterschiedliche Mechanismen der Schmerzwahrnehmung aber auf ähnliche Mediatoren der im Alltag wahrgenommenen Beeinträchtigung hin.

\section{Schmerz bei Kindern und im Alter}

\section{SY02 - Schmerztherapeutische Versorgung von Kindern und Jugendlichen in Deutschland}

F. Ebinger', N. Draheim² ${ }^{2}$ M. Hartwig ${ }^{3}$, M. Blankenburg ${ }^{4}$

${ }^{1}$ St. Vincenz Krankenhaus Paderborn, Klinik für Kinder- und Jugendmedizin, Paderborn, Deutschland, ${ }^{2}$ Deutsches Zentrum für Kinder- und Jugendrheumatologie, Garmisch-Partenkirchen, Deutschland, ${ }^{3}$ Zentrum für Kinderschmerztherapie, Hamburg, Deutschland, ${ }^{4}$ Klinikum Stuttgart, Pädiatrie 1, Stuttgart, Deutschland

Kinder und Jugendliche leiden in zunehmender Häufigkeit unter chronischen oder chronisch-rezidivierenden Schmerzen. Angesichts dieses zunehmenden Bedarfs ist die ambulante und stationäre schmerztherapeutische Versorgungssituation für Kinder und Jugendliche in Deutschland unzureichend. Betroffene, deren Eltern aber auch betreuende Ärzte suchen oft lange nach entsprechenden Einrichtungen. Die bestehenden Angebote haben zudem sehr unterschiedliche Strukturen und inhaltliche Standards. Der Arbeitskreis Schmerz bei Kindern und Jugendlichen der Deutschen Schmerzgesellschaft hat deswegen eine Bestandsaufnahme der bestehenden Einrichtungen mit schmerztherapeutischen Angeboten für Kinder und Jugendliche begonnen. Dabei wurde das therapeutische Angebot aber auch besondere Indikationen einzelner Zentren erfragt. Ziel ist es, eine pädiatrisch-schmerztherapeutische Versorgungslandkarte zu erstellen, die sowohl Patienten als auch Ärzten und Behandlern als Orientierungshilfe dient. Das Ergebnis dieser Erhebung wird im ersten Vortrag dargestellt. Im zweiten und drittem Vortrag der Sitzung werden am Beispiel des Zentrums für Kinderschmerztherapie und Palliativmedizin in Hamburg bzw. der Station für pädiatrische Psychosomatik und Schmerztherapie am Olgahospital in Stuttgart Aufbau und Struktur eines ambulanten und eines stationären Versorgungsangebotes für Kinder und Jugendliche dargestellt. Dabei geht es um strukturelle Voraussetzungen, um sinnvolle Konzepte, aber auch um eventuelle Probleme beim Aufbau.

1. Dr. Nicole Draheim; Garmisch-Partenkirchen: Versorgungslandkarte „Schmerztherapeutische Versorgung von Kindern und Jugendlichen in Deutschland“

2. Dr. Maite Hartwig; Hamburg: Aufbau und Struktur einer ambulanten schmerztherapeutischen Praxis für Kinder und Jugendliche

3. PD Dr. Markus Blankenburg; Stuttgart: Aufbau und Struktur einer stationären schmerztherapeutischen Einrichtung für Kinder und Jugendliche

\section{Kopfschmerz}

\section{SY03 - Von der Pathophysiologie zur Diagnostik: Neuroplastizität bei Kopfschmerzerkrankungen}

T. Sprenger', L. Neeb ${ }^{2}$, D. Holle-Lee ${ }^{3}$

'DKD Helios Klinik Wiesbaden, Fachbereich Neurologie, Wiesbaden, Deutschland, ${ }^{2}$ Klinik und Hochschulambulanz für Neurologie, Kopfschmerzzentrum Charité, Berlin, Deutschland, ${ }^{3}$ Universitätsklinikum Essen (AöR), Neurologische Klinik, Westdeutsches Kopfschmerzzentrum, Essen, Deutschland

Vortragstitel:

1. Klinische Aspekte zur Bildgebung bei Kopfschmerzen. - Till Sprenger, Wiesbaden

2. fMRT und Co: Wie kann uns die Bildgebung zu einem besseren Verständnis der Pathophysiologie verhelfen? - Lars Neeb, Berlin

3. Blinkreflex und Co: Mechanismen der Kopfschmerzchronifizierung. Dagny Holle-Lee, Essen

Kopfschmerzen können zu einem erheblichen individuellen „Burden of disease“ führen und durch wiederholte ärztliche Vorstellungen und Durchführung vieler diagnostischer Verfahren hohe gesundheitsmedizinische Kosten verursachen. Oftmals wird mehrfach eine Bildgebung 
von Kopf und HWS durchgeführt, wofür häufig keine Indikation besteht. Durch irrelevante Zufallsbefunde können wiederum weitere Kosten und Belastungen für die Patienten entstehen. Die zugrunde liegende Pathophysiologie der Entstehung und Chronifizierung von Kopfschmerzen ist bislang weitestgehend unbekannt.

Ein erster Schwerpunkt dieses Symposiums soll es sein, die Indikation für Bildgebung bei Kopfschmerzen zu beleuchten und häufige (Zufalls-)Befunde sowie deren Wertigkeit zu diskutieren. Vor allem bei Patienten mit Migräne mit Aura zeigen sich häufig T2 Hyperintensitäten im Marklager, die zu Unsicherheiten bzgl. des weiteren Procederes führen.

Der zweite Schwerpunkt des Symposiums befasst sich mit neuroplastischen Veränderungen bei verschiedenen (chronischen) Kopfschmerzerkrankungen, die eine wichtige Rolle in deren Pathophysiologie spielen und möglichweise auch an Chronifizierungsprozessen beteiligt sind. Zudem werden pathophysiologische Grundlagen bzgl. elektrophysiologische Veränderungen sowie Alterationen in Bildgebungsstudien beleuchtet. Mögliche zukünftige Interventionsmöglichkeiten zur Verhinderung der Chronifizierung von Kopfschmerzen werden aufgezeigt.

Aufgrund des breit angelegten Themenbereiches richtet sich das Symposium sowohl an den Kliniker, als auch an Wissenschaftler, die sich näher mit der Diagnostik und Pathophysiologie von chronischen Kopfschmerzen beschäftigen.

Herr Prof. Sprenger wird Diagnosealgorithmen vorstellen, die helfen sollen Patienten zu identifizieren, die eine Bildgebung benötigen. Ferner werden häufige Zufallsbefunde besprochen und ein rationales Procedere diskutiert. Er wird dabei Bezug nehmen auf neueste klinische Studien. Dr. Neeb wird die neusten Bildgebungsstudien zu strukturellen und funktionellen Veränderungen bei Kopfschmerzen vorstellen und mögliche Folgen bzw. Ursachen der Chronifizierung analysieren. Frau PD Holle-Lee wird anhand tierexperimenteller und humaner Studiendaten elektrophysiologische Grundlagen der Kopfschmerzchronifizierung beleuchten und mögliche daraus resultierende therapeutische Interventionsmöglichkeiten aufzeigen.

\section{Schmerz nach Operationen}

\section{SY04 - Professionell interprofessionell handeln: Wer darf was in der Akutschmerztherapie}

V. Deichert ${ }^{1}$, A. Moroder ${ }^{2}$, J. Erlenwein ${ }^{3}$

'Universitätsklinikum Münster, Klinik für Anästhesiologie, Intensivmedizin und Schmerztherapie, Münster, Deutschland, ${ }^{2}$ Rechtsanwaltsgesellschaft Ehlers, Ehlers \& Partner, München, Deutschland, ${ }^{3}$ Universitätsmedizin Göttingen, Klinik für Anästhesiologie, GF Schmerzmedizin, Göttingen, Deutschland

Die Akutschmerztherapie ist eine interprofessionelle und interdisziplinäre Aufgabe! Eine gute und vertrauensvolle Zusammenarbeit der Akteure ist Voraussetzung einer effektiven und ausreichenden Schmerztherapie. Jedoch ergeben sich aus dem Spannungsfeld zahlreicher Schnittstellen und Interaktionen zwischen medizinischen Fachabteilungen, aber auch zwischen ärztlichen und pflegerischen Mitarbeitern, Fragen nach Verantwortung und Haftung sowie dem rechtlichen Rahmen zur Delegation ärztlicher Tätigkeiten. Diese Fragen gewinnen aktuell an Brisanz, da vor dem Hintergrund des wachsenden ökonomischen Drucks in vielen Kliniken, besonders im Akutschmerzdienst, ärztliche Aufgaben zunehmend durch Pflegende übernommen werden. Grundsätzlich gilt in Deutschland der Arztvorbehalt, was die Abgrenzung von professionsspezifischen Aufgaben in diesem Team notwendig macht. Daraus ergeben sich auch Implikationen an die Organisation der Schmerztherapie im Krankenhaus. Diese fließen derzeit zur Sicherung der Versorgungsqualität in der spezialisierten Akutschmerztherapie auf Arbeitskreisebene in die Erarbeitung von Empfehlungen zu personellen, qualifikatorischen und organisatorischen Anforderungen an Akutschmerzdienste.
Frau Professor Pogatzki-Zahn stellt die derzeitige Praxis der interdisziplinären Zusammenarbeit, sowie die Interaktion zwischen Ärzten und Pflegenden in der Akutschmerztherapie vor. Dabei stellt sie zum einen die Problematik hinsichtlich Verantwortung und Haftung, die sich z. B. aus der Nutzung von Behandlungsstandards auf der Normalstation ergibt, dar; zum anderen leitet sie Fragen zur rechtlichen Einordnung der interdisziplinären und interprofessionellen Zusammenarbeit in der Akutschmerztherapie und zu Voraussetzungen der Delegation ärztlicher Tätigkeiten an Pflegende im Akutschmerzdienst ab. Frau Dr. Moroder wird in ihrem Vortrag aus medizinjuristischer Sicht einen Überblick zu den rechtlichen Rahmenbedingungen und zu Haftungsfragen in diesem Kontext geben. Herr Dr. Erlenwein stellt die sich aus diesem rechtlichen Rahmen ergebenden Anforderungen an die Organisation der Akutschmerztherapie und Modelle zu deren Finanzierung dar. Als Grundlage dienen die sich derzeit in Erarbeitung befindlichen Empfehlungen für Akutschmerzdienste, welche entgegen ökonomischer Prämissen die Behandlungsqualität in der spezialisierten Versorgung durch Akutschmerzdienste sicherstellen sollen.

\section{Diagnostisches Procedere}

\section{SY05 - Schmerzen innerhalb und außerhalb der Röhre - Möglichkeiten der modernen MR-Methodik und Elektrophysiologie für Forschung und Klinik}

\section{Segelcke' ${ }^{1}$, T. Schmidt-Wilcke ${ }^{2}$, W. Mager ${ }^{3}$}

${ }^{1}$ Universitätsklinikum Münster, Klinik für Anästhesiologie, operative Intensivmedizin und Schmerztherapie, Münster, Deutschland, ${ }^{2}$ Berufsgenossenschaftliches Universitätsklinikum Bergmannsheil GmbH, Klinik für Neurologie, Bochum, Deutschland, ${ }^{3}$ Medizinische Fakultät Mannheim, Lehrstuhl für Neurophysiologie, CBTM, Mannheim, Deutschland

1. Schmerz im Nagerhirn - Welche Erkenntnisse gibt es aus der Grundlagenforschung? - Daniel Segelcke, Münster

2. Neues zur Hirnfunktion und Neurochemie des chronischen Schmerzes - Tobias Schmidt-Wilcke, Bochum

3. Stand der Technik nozizeptiv evozierter Potentiale - Walter Magerl, Mannheim

Durch die funktionelle Bildgebung des Gehirns konnten in den letzten Jahren neue Einblicke in die zentrale Verarbeitung der Schmerzwahrnehmung gewonnen werden. Dies hat unser Verständnis der Schmerzchronifizierung erheblich erweitert. Neuere Techniken schließen in diesem Zusammenhang die funktionelle Kernspintomographie im Ruhezustand (d. h. die Darstellung von funktionellen Gehirnkonnektivitäten), die Spektroskopie (d.h. Darstellung der Neurochemie, z. B. im GABA- oder opioidergen System) sowie die Bildgebung im Tiermodell ein. Auch die Auswirkung einer medikamentösen Beeinflussung und der damit verbundenen Änderung der Schmerzwahrnehmung sind Teil der aktuellen Forschung. Aufgrund der Vielzahl an Daten aus diesen Untersuchungen ergibt sich jedoch auch die Frage, welche Hirnareale überhaupt wichtig für die untersuchten Schmerzprozesse sind? Und sind dieselben Areale relevant für akute und chronische Schmerzen? Daher ist ein kritischer Umgang mit Informationen in diesem Bereich besonders essentiell für eine korrekte Interpretation der Ergebnisse. Dieses Symposium soll schwerpunktmäBig einen Einblick in den Bereich der tier- und humanexperimentellen Forschung geben und aufzeigen welche neuen Erkenntnisse die funktionelle Bildgebung und die Spektroskopie unter zu Hilfenahme moderner Analyseverfahren bereitstellt und wie mit diesen Daten kritisch umgegangen werden soll. Aufgrund des breiten Themenspektrums richtet sich das Symposium sowohl an Kliniker, als auch an Grundlagenwissenschaftler, die sich mit den Möglichkeiten der modernen Bildgebung und Technik evozierter Potentiale kritisch auseinandersetzten möchten. Herr Dr. Daniel Segelcke gibt in seinem Vortrag einen Überblick über aktuelle Erkenntnisse der funktionalen Bildgebung aus der tierexperimentellen Grundlagenforschung und zeigt welche Chancen diese Methoden für die Schmerzforschung bietet. Herr Prof. Dr. Tobias Schmidt-Wilcke legt 
den Schwerpunkt seines Vortrages auf humane Studien, die zum einen die funktionelle Konnektivität verschiedener schmerzverarbeitender Hirnregionen darstellt und zum anderen eine mögliche Korrelation zwischen lokalen GABA Konzentrationen mit der individuellen Schmerzschwelle aufzeigt. Der dritte Vortrag (Magerl, Mannheim) gibt einen Überblick über die derzeit verfügbaren Methoden zur Prüfung der nozizeptiven Bahnen mittels evozierter Potentiale. Es wird ein Überblick gegeben über verschiedene Methoden zur differenzierten Auslösung schmerzbezogener Aktivierung durch natürliche (Hitze, Kälte, mechanisch, chemisch) und elektrische Stimulation und damit dem Monitoring funktionell verschiedener Subgruppen nozizeptiver Afferenzen zur differenzierten Darstellung von A-Delta (1.Schmerz) und C-Faser-bezogener Aktivierung (2.Schmerz) durch verschiedene Typen von späten und ultraspäten evozierten Potentialen (LEPs, CHEPs, CCEPs, PEPs, PREPs ...) zur Darstellung der Integrität, Beeinträchtigung oder Überempfindlichkeit der nozizeptiven Projektionsbahnen.

\section{Bewegungstherapie und manuelle Medizin}

\section{SY06 - Adipositas - Proinflammatorische Zytokine - Chronischer Schmerz Einflussmöglichkeiten über Vagus- und körperliche Aktivierung}

\section{Moser' ${ }^{1}$, B. Schultes ${ }^{2}$, U. Hamberger ${ }^{3}$}

${ }^{1}$ Medizinische Universität Graz, Physiologie, Graz, Österreich, ${ }^{2}$ eSwiss Medical \& Surgical Center, St. Gallen, Schweiz, ${ }^{3}$ Klinikum Landsberg, Schmerzzentrum, Landsberg, Deutschland

1. Adipositas und Chronischer Schmerz - Die Rolle proinflammatorischer Zytokine; Bernd Schultes, St. Gallen $(\mathrm{CH})$

2. Vagus Aktivierung und Makrophagenaktivität; Maximilian Moser, Graz (A)

3. Einflussmöglichkeiten körperlicher Aktivierung; Ulrich Hamberger, Landsberg am Lech

Menschen mit Adipositas weisen häufig chronische Schmerzen auf. Dabei spielen nicht nur die mechanische Belastung, sondern auch hormonelle und inflammatorische Signale eine entscheidende Rolle. Die aus dem Bereich des viszeralen Fettgewebes frei gesetzen proinflammatorischen $\mathrm{Zy}$ tokine können hierbei einen stark Schmerzmodulierenden- bzw. verstärkenden Einfluss ausüben.

Bei der Regulation der daraus resultierenden „silent inflammation“ wird dem Vagus eine zentrale entzündungs- und schmerzreduzierende Funktion zugesprochen. Afferente Anteile des Vagus besitzen Rezeptoren für entzündungsfördernde Botenstoffe. Efferente Anteile können direkt über die Ausschüttung von Acetylcholin die Makrophagenaktivität im Entzündungsbereich vermindern.

Körperliche Aktivierung kann sowohl direkt den Abbau viszeralen Fettgewebes bewirken, als auch den Vagus über Steigerung der parasympathischen Aktivität beeinflussen. Muskelaktivität wirkt zusätzlich durch die Freisetzung von Myokinen, proinflammatorischen Zytokinen entgegen. Herr Professor Schultes wird zunächst die Zusammenhänge zwischen Adipositas, viszeralem Fettgewebe, proinflammatorischen Zytokinen und chronischen, insbesondere muskuloskelettalen Schmerzen erläutern. Die Entzündungs - und somit Schmerzreduzierende Funktion einer Vagus Aktivierung wird im anschliessenden Teil vorgestellt. Herr Professor Moser geht hierbei auch auf die diagnostische Möglichkeit der Messung der parasympathischen Aktivität durch die Herzrhythmusflexibiltät ein. Zum Abschluss wird U. Hamberger das Potential körperlicher Aktivierung, sowohl in Anbetracht viszeraler Fettansammlung, als auch hinsichtlich der Beeinflussung des autonomen Nervensystems und der Reduzierung proinflammatorischer Zytokine, vorstellen. Insbesonders wird hierbei auf die Bedeutung von Ausdauer- und Kraft- orientierten Trainingsformen eingegangen.

\section{Experimentelle Modelle und Pathophysiologie}

\section{SY08 - Elektrisch evozierte Schmerz-assoziierte Potentiale bei der Diagnostik von Neuropathien und bei der Untersuchung der konditionierten Schmerzmodulation}

\section{E. Enax-Krumova' ${ }^{1}$ O. Höffken ${ }^{1}$, N. Üceyler ${ }^{2}$}

'BG Universitätsklinikum Bergmannsheil, Neurologische Klinik, Bochum, Deutschland, ${ }^{2}$ Universitätsklinikum Würzburg, Neurologie, Würzburg, Deutschland

1. Die konditionierte Schmerzmodulation - methodische Aspekte und klinische Relevanz (Dr. med. E. Enax-Krumova, Bochum)

2. Ein neues Modell zur Untersuchung der konditionierten Schmerzmodulation mittels elektrisch evozierter Schmerz-assoziierter Potenziale (PD Dr. med. O. Höffken, Bochum)

3. Elektrisch evozierte Schmerz-assoziierte Potenziale - klinische Relevanz in der Diagnostik von Neuropathien (PD Dr. med. N. Üçeyler, Würzburg)

Die konditionierte Schmerzmodulation (conditioned pain modulation, CPM) ist eine Methode zur experimentellen Erfassung der endogenen Schmerzmodulation. Dabei wird der Effekt eines noxischen konditionierenden Reizes auf die Intensität eines vorher applizierten noxischen Testreizes untersucht. Eine erste Untersuchung an Patienten mit schmerzhafter diabetischer Polyneuropathie zeigte, dass der Therapieeffekt nach Duloxetin-Gabe vom Zustand der endogenen Schmerzmodulation vor Therapiebeginn (erfasst mittels CPM) abhängt. Diese Ergebnisse unterstreichen die klinische Relevanz der Methode. In Anbetracht der Vielfalt an beschriebenen Untersuchungsprotokollen mit verschiedenen Test- und konditionierenden Stimuli stellt sich die Frage, welches am besten für den klinischen Einsatz hinsichtlich Validität, Reliabilität und Praktikabilität geeignet ist. Ein optimales CPM-Messprotokoll sollte zudem einfach umsetzbar im klinischen Alltag sein sowie auch möglichst auch objektive Parameter, zusätzlich zu den Angaben der Schmerzintensität, erfassen. Elektrisch evozierte Schmerz-assoziierte Potenziale (pain-related evoked potentials, PREP) bieten eine Möglichkeit die Konduktivität A-delta vermittelter Nervenfaserbahnen zu untersuchen. Der dabei applizierte Stromimpuls hat eine definierte Stärke und die verursachte subjektive Schmerzhaftigkeit kann auf einer numerischen Ratingskala individuell angegeben werden. Das Reizantwortpotenzial kann zudem objektiv gemessen werden. Korrelationen mit anderen Methoden, die z. B. die Morphologie (intraepidermale Nervenfaserdichte) oder die Funktion (thermische Wahrnehmungsschwellen) der kleinen Nervenfasern untersuchen, geben Hinweise darauf, dass PREP von diagnostischer Relevanz sein könnte.

Frau Dr. Enax-Krumova stellt in ihrem Vortrag die aktuellen Forschungsergebnisse über das CPM-Untersuchungsparadigma, methodische Besonderheiten sowie die klinische Relevanz der CPM-Erfassung. Herr Priv.Doz. Dr. Höffken wird Daten über die Reliabilität der PREP vorstellen sowie ein neues Protokoll zur Untersuchung der CPM mittels PREP präsentieren.Frau Priv.-Doz. Dr. Üçeyler wird schließlich die aktuelle Studienlage zusammenfassen, welche Relevanz PREP für den klinischen Alltag in der Diagnostik von Neuropathien haben. 


\section{Translationale Schmerzforschung}

\section{SY09 - Die altbekannten „transient receptor potential“ (TRP)- Schmerzkanäle - noch Targets für neue Schmerzmedikamente?}

H. Rittner', B. Oehler ${ }^{2}$, M. Schmidt ${ }^{3}$

${ }^{1}$ Klinik und Poliklinik für Anästhesiologie, Zentrum für Operative Medizin, Universitätsklinikum Würzburg, Würzburg, Deutschland, ${ }^{2}$ Universitätsklinikum Würzburg, Klinik und Poliklinik für Anästhesiologie, Würzburg, Deutschland, ${ }^{3}$ Max Planck Institute of Experimental Medicine, Göttingen, Deutschland

1. Neue Botenstoffe für TRP-Kanäle auf Nozizeptoren - mehr als Capsaicin, Beatrice Oehler, Würzburg

2. Interaktionspartner von TRP-Kanälen - anders als Capsaicin, Manuela Schmidt, Göttingen

3. Aktueller Stand: TRP Kanalblocker zur Analgesie bei Patienten - gegen Capsaicin, Heike Rittner, Würzburg

Vor knapp 20 Jahren wurde von D. Julius der Capsaicinrezeptor (transient receptor potential vanilloid 1 - TRPV1) als einer der ersten Schmerzrezeptoren entdeckt. Bis zu diesem Zeitpunkt war die Vorstellung von Schmerzentstehung und molekularen Mechanismen eher rudimentär. Ebenso ist TRP ankyrin-1 (TRPA1) seit 2003 als Kältesensor und Rezeptor für irritierende Substanzen aus der Arbeitsgruppe von A. Patapoutian bekannt. Zwischen der Erstbeschreibung und heute liegen viele Publikationen und klinische Studien, allerdings ist der große Durchbruch als Target von Schmerzmedikamenten bisher ausgeblieben. Diese Sitzung soll im ersten Teil neueste Erkenntnisse zu diesen beiden Schmerzrezeptoren von der „bench zur bedsite“ aufzeigen. Im zweiten Teil sollen Hindernisse und neue Chancen auf dem Weg zur Entwicklung von neuen Schmerzmedikamenten, die TRP Kanäle als Target haben, analysiert werden.

Im ersten Vortrag von B. Oehler wird eine neue Gruppe von Botenstoffen aus der Membran, die durch reaktive Sauerstoffspezies entstehen [2], vorgestellt. Diese werden bei Entzündungen und bei Arthritis freigesetzt und aktivieren TRPA1. Durch Abfangen dieser Botenstoffe oder mit TRP-Blocker können Entzündungsschmerzen gelindert werden [4]. Der Vortrag von $M$. Schmidt wird neueste Grundlagenforschung zu spezifischen Interaktionspartnern von TRPA1 und TRPV1 Kanälen vorstellen [1]. Derartige Interaktionspartner modulieren die Aktivität der TRP Kanäle und könnten daher alternative Angriffspunkte für die Schmerztherapie darstellen. Im dritten Abschnitt werden von H. Rittner die klinischen Studien zu TRP Blockern beleuchtet [3]

Dieses Symposium soll Nachwuchswissenschaftlern die Gelegenheit geben, Ihre spannenden Forschungsergebnisse einem breiten Publikum zu vorzustellen. Zielgruppen des Symposiums sind sowohl interessierte Kliniker als auch Grundlagenwissenschaftler, denn klinische und grundlagenwissenschaftliche Studien werden von klinischen Wissenschaftlern diskutiert.

\section{Literatur}

1. Avenali, L.et al. (2014). Annexin A2 regulates TRPA1-dependent nociception. J Neurosci 34, 14506-14516.

2. Hackel, D., et al. (2013). The connection of monocytes and reactive oxygen species in pain. PLoS One 8 , e63564.

3. Harteneck, C., et al. (2011). Synthetic modulators of TRP channel activity. Adv Exp Med Biol 704, 87-106.

4. Oehler, B., et al. (2015) ApoA-I-mimetisches Peptid und E06 mAb als neue Targets in OxPAPC-induzierter Hyperalgesie in Ratten. Anästh Intensivmed 56, 340-390

\section{Epidemiologie und Genetik}

\section{SY10 - Kopfschmerzen und Genetik - was wissen wir, was nutzt uns dieses Wissen und was bringt die Zukunft?}

R. Malik', E. Auffenberg', U. Hedrich², T. Freilinger ${ }^{3}$

'Institut für Schlaganfall- und Demenzforschung, München, Deutschland,

${ }^{2}$ Hertie-Institut für Klinische Hirnforschung, Tübingen, Deutschland, ${ }^{3}$ Universitätsklinikum Tübingen, Abteilung Neurologie mit Schwerpunkt Epileptologie, Tübingen, Deutschland

1. Migräne: häufige und ganz seltene Formen und was wir zu ihrer Genetik wissen und (noch) nicht wissen. Tobias Freilinger, Tübingen

2. Mehr als Migräne: welche Rolle spielen genetische Risikofaktoren für häufige Komorbiditäten der Migräne? Rainer Malik, München

3. Genetische Tiermodelle für Migräne - Stand der Forschung und eigene Daten. Eva Auffenberg und Ulrike Hedrich, München \& Tübingen Das immer wieder diskutierte Thema „Kopfschmerzen und Genetik“ hat gerade aktuell eine hohe Relevanz, haben genetische Befunde doch ein großes Potential als mögliche „Biomarker“. Gerade was die Migräne betrifft, hat unser Verständnis der genetischen Basis in den letzten wenigen Jahren sprunghaft zugenommen. Mit diesem zunehmenden Wissen Schritt zu halten und die immer neuen Befunde kritisch einzuordnen, ist gerade für den Nicht-Spezialisten schwierig. Das Symposium möchte daher für Kliniker, aber auch (Kopf-) Schmerz-Forscher einen breiten aktuellen Überblick zu ,Kopfschmerzen und Genetik' geben. In einem ersten Schwerpunkt soll verständlich gemacht werden, was wir im Moment über die Genetik der Migräne wissen; es wird um sehr seltene Formen wie die hemiplegische Migräne gehen, aber auch um die häufigen Formen, wie wir sie in der täglichen klinischen Praxis sehen. Neben einer Diskussion pathophysiologischer Implikationen soll die Relevanz der Befunde für Diagnose, Therapie und Prognose beleuchtet und ein Ausblick auf zukünftige Entwicklungen (Stichwort: personalisierte Medizin; Genetik anderer primärer Kopfschmerzerkrankungen) gewagt werden. Anknüpfend hieran wird ein zweites Referat beleuchten, wie die Genetik sich häufigen und klinisch relevanten Komorbiditäten der Migräne näher kann. Ein letzter Schwerpunkt wird sich dem Thema aus pathophysiologisch-funktionellem Blickwinkel nähern und genetisch definierte Migräne-Tiermodelle diskutieren. Herr PD Dr. Freilinger beschäftigt sich mit Klinik und Genetik paroxysmaler neurologischer Erkankungen; er wird den derzeitigen Wissensstand der Migräne-Genetik in verständlicher Form aufbereiten. Anhand einer eigenen Pilotstudie wird er u. a. aufzeigen, wie genetische Befunde in neue translationale Therapieansätze münden können. Herr Dr. Rainer Malik hat selbst entscheidende Beiträge zur Entschlüsselung der genetischen Basis häufiger neurologischer Erkrankungen wie Migräne oder Schlaganfall geliefert; er wird der Frage nachgehen, inwieweit die Genetik häufige Komorbiditäten der Migräne erklären kann, mit einem Fokus auf das brisante Thema Migräne und Schlaganfall. Frau Dr. Eva Auffenberg und Frau Dr. Ulrike Hedrich beschäftigen sich in tierexperimentellen Studien mit der corticalen Erregbarkeit bei Migräne (in Abgrenzung zur Epilepsie) und werden neben einem Überblick zu genetischen Tiermodellen erste eigene Daten vorstellen.

\section{Varia}

\section{SY11 - Stiefkinder der Schmerzversorgung - bisher wenig beachtete Bereiche der Akutschmerztherapie}

C. Schwarze', J. Erlenwein'2 , J. Filitz ${ }^{3}$

'Uniklinikum Münster, Klinik für Anästhesiologie und postoperative Intensivmedizin, Münster, Deutschland, ${ }^{2}$ Universitätsmedizin Göttingen, Klinik für Anästhesiologie, GF Schmerzmedizin, Göttingen, Deutschland, ${ }^{3}$ Medizinische Hochschule Hannover, Klinik für Anästhesiologie und Intensivmedizin, Hannover, Deutschland 
1. „Schmerz nach ambulanten Operationen“ - Herr Christian Schwarze

2. „Schmerz bei nicht-operativen Patienten im Krankenhaus“ - Dr. med. Joachim Erlenwein

3. „Procedure Related Pain“ - Dr. med. Jörg Filitz

Der Fokus von Initiativen zur Verbesserung der Qualität der Schmerztherapie lag bisher vorrangig auf der Behandlung postoperativer Schmerzen im Krankenhaus. Jedoch gibt es in der Patientenversorgung auch andere Bereiche, in denen Patienten teils erheblich Schmerzen erleiden, die bisher in der Betrachtung wenig Beachtung gefunden haben. Gesundheitspolitisch angetrieben stieg beispielsweise in den letzten Jahren der Anteil ambulanter Operationen stark an, zu denen jedoch bisher Daten zur Qualität der Schmerztherapie fehlen. Wie unter anderem durch das Projekt „Schmerzfreies Krankenhaus" nachgewiesen wurde, gibt es deutliche Hinweise, dass gerade auch in nicht-operativen Fachbereichen Defizite in der Schmerzversorgung bestehen. Ein Großteil ambulanter und stationärer Patienten erlebt Schmerzen im Rahmen von Untersuchungen, Punktionen, Verbandswechseln oder Wundreinigungen. Während es für Kinder inzwischen einige verbreitete Ansätze, wie beispielsweise das EMLA-Pflaster vor Punktionen, Ablenkung- oder Suggestionstechniken gibt, existieren für diese teils sehr starken Schmerzen bei Erwachsenen meist keine spezifischen Konzepte. Herausforderungen ergeben sich oft auch dadurch, dass diese Prozeduren in der Regel nicht in Überwachungsbereichen erfolgen, sondern auf Normalstationen, oder in der Praxis und Ambulanz. Herr Schwarze wird seinen Schwerpunkt auf aktuelle Versorgungsstudien zur Effektivität der Schmerztherapie nach ambulanten Operationen legen. Dabei gibt er einen Überblick über mehrere operative Fachdisziplinen, besonders schmerzhafte Eingriffe und deren Schmerzverläufe, auch zu dem Zeitpunkt, wenn kein Fachpersonal mehr zu erreichen ist - nämlich zuhause. Dabei präsentiert er mögliche Risikofaktoren für langanhaltende Schmerzen nach ambulanten Operationen und deren Auswirkungen auf die Lebensqualität. Herr Erlenwein wird unteranderem anhand von Zertifizierungsdaten darstellen, für welche Patienten konservativer Abteilungen im Krankenhaus sich derzeit besondere Versorgungsprobleme ergeben Auch wird er erste Daten aus der Entwicklung eines Tools für nicht-operative Patienten präsentieren, welches analog zu QUIPS (Qualitätsverbesserung in der postoperativen Schmerztherapie) die Bewertung der Qualität der Schmerztherapie aus Patientenperspektive ermöglicht. Herr Filitz wird die Problematik von eingriffsspezifischen Schmerzen z. B. im Rahmen von Untersuchungen, Punktionen, Verbandswechseln oder Wundreinigungen darstellen. Insbesondre gilt es hier einfache Konzepte und Techniken zu diskutieren, mit denen der nicht auf Anästhesie-spezialisierte Arzt in seinem Arbeitsumfeld die Schmerzen seiner Patienten reduzieren kann. Gerade hinsichtlich von Untersuchungen gilt es, diese von einer Analgosedierung abzugrenzen.

\section{Muskuloskelettale Schmerzen}

\section{SY12 - Aktuelle Erkenntnisse zur Pathophysiologie, Diagnostik und Therapie des Gelenkschmerzes (ein Beitrag zum 2016 Global Year Against Pain in the Joints)}

H. Schaible ${ }^{1}$, H. Locher ${ }^{2}$, H. Casser ${ }^{3}$

'Universitätsklinikum Jena, Institut für Physiologie 1/Neurophysiologie, Jena, Deutschland, ${ }^{2}$ Zentrum für Schmertherapie und Manuelle Medizin, Tettnang, Deutschland, ${ }^{3}$ DRK Schmerz-Zentrum Mainz, Mainz, Deutschland

Vorsitz: Hans-Georg Schaible, Jena

1. Erkenntnisse zu den Zusammenhängen von Inflammation und Nozizeption beim Gelenkschmerz Hans-Georg Schaible, Jena

2. Richtungsweisende anamnestische und klinische Befunde einschließlich Bildgebung bei Gelenkschmerzen und Abgrenzung vom myofasziellen Schmerz Hermann Locher, Tettnang

3. Evidenzbasierte Behandlung des Gelenkschmerzes aus schmerztherapeutischer Sicht, Hans-Raimund Casser, Mainz
Muskuloskelettale Schmerzen zählen zu den häufigsten chronischen Schmerzen. Besonders betroffen ist der Rücken, aber auch periphere Gelenke (hier ist die Arthrose am häufigsten). In jüngster Zeit gibt es neue Impulse in Forschung und Klinik, die mittelfristig hoffentlich zu besseren therapeutischen Ergebnissen führen werden. Erstens ändert sich die Vorstellung über die Pathophysiologie der „,degenerativen“ Erkrankungen des muskuloskelettalen Systems. Zweitens finden neurophysiologische Konzepte der Schmerzentstehung Eingang in die klinische Diagnostik und Praxis. Drittens werden in diesem Bereich schmerztherapeutische Konzepte angewandt, die das bio-psycho-soziale Schmerzmodell berücksichtigen.

Viele Erkrankungen des muskuloskelettalen Systems, z. B. die Arthrose, wurden lange Zeit als „degenerativ“ angesehen, wobei mechanischen Faktoren die wichtigste pathogenetische Rolle zugeschrieben wurde. In den letzten Jahren wird immer deutlicher, dass bei solchen Erkrankungen auch entzündliche Vorgänge und metabolische Faktoren (z.B. das metabolische Syndrom) wesentliche pathogenetische Mechanismen sind. Im ersten Beitrag (Schaible) werden aktuelle Befunde dargestellt, die eine wichtige Rolle entzündlicher und metabolischer Faktoren bei der Entstehung der Schmerzen bei „degenerativen“ muskuloskelettalen Erkrankungen, speziell der Arthrose, zeigen.

Die Diagnostik von Schmerzen in muskuloskelettalen System hat die Aufgabe, Schmerzen hinsichtlich der verursachenden Strukturen und der daran beteiligten neurophysiologischen Schmerzmechanismen zu identifizieren. Der zweite Beitrag (Locher) wird anhand typischer Beispiele aus der Praxis zeigen, in welcher Weise der klinische Befund, der durch gezielte manuelle Untersuchung erhoben werden kann, neben der Bildgebung Rückschlüsse auf den Schmerzort und die neurophysiologischen Schmerzmechanismen geben kann.

Der dritte Beitrag (Casser) wird die evidenzbasierten Behandlungsmöglichkeiten besonders bei Arthrosen im Laufe der Erkrankung darstellen. Da in näherer Zukunft mit einer kausalen Therapie der Arthrose aufgrund ihrer multifaktoriellen Genese nicht zu rechnen ist, ist eine strukturierte Vorgehensweise aus schmerztherapeutischer Sicht (dem bio-psycho-sozialen Modell folgend) erforderlich. Es wird ferner herausgestellt werden, in welche Richtung die schmerztherapeutischen Konzepte weiterentwickelt werden müssen.

\section{Translationale Schmerzforschung}

\section{SY13 - Der gestresste Rücken: Psychobiologische Zusammenhänge von Stress und Rückenschmerz}

S. Mense', J. Tesarz ${ }^{2}$, M. Noll-Hussong ${ }^{3}$, W. Eich ${ }^{4}$

'Universität Heidelberg, Mediz. Fakultät Mannheim, CBTM, Neuroanatomie, Mannheim, Deutschland, ${ }^{2}$ Universitätsklinik Heidelberg, Sektion Muskuloskelettaler Schmerz, Heidelberg, Deutschland, ${ }^{3}$ Universitätsklinikum Ulm, Klinik und Poliklinik für Psychosomatische Medizin und Psychotherapie, Ulm, Deutschland, ${ }^{4}$ Klinik für Allgemeine Innere Medizin und Psychosomatik/Uniklinik Heidelberg, Sektion Integrierte Psychosomatik (Schwerpunkt Bewegungssystem), Heidelberg, Deutschland

Stress hat einen starken Einfluss auf die biologischen und psychosozialen Ebenen der Schmerzwahrnehmung. So ist bekannt, dass Distress die Entwicklung von nicht-spezifischem Rückenschmerz fördern und seine Intensität verstärken kann. Über welche Mechanismen Stress sich auf die Entstehung von Rückenschmerzen auswirkt, ist weitgehend unklar. Inhalt dieses Symposiums soll eine differenzierte Darstellung über das aktuelle Wissen um mögliche Zusammenhänge zwischen Stress und chronischem Schmerzerleben, insbesondere chronischen Rückenschmerzen, sein. Im Vortrag von S. Mense soll zunächst auf den Einfluss von wiederholten Stresserfahrungen auf die spinale Prozessierung von nozizeptiven Informationen eingegangen werden. Hierfür wurde tierexperimentell der Einfluss von Stress auf sensorische Neurone im Rückenmark von Ratten untersucht. Die Nager wurden hierfür zunächst wiederholt einem Immo- 
bilisationsstress unterworfen. Anschließend wurden die Entladungen von Hinterhornneuronen im lumbalen Rückenmark mit Mikroelektroden registriert. Die Neurone der gestressten Tiere wiesen gesteigerte Antworten bei Reizung der Rückenweichteile und eine verstärkte Ruheaktivität auf. Die Steigerung der neuronalen Antworten auf mechanische Reize könnte die stressbedingten stärkeren Rückenschmerzen mit erklären, die gesteigerte Ruheaktivität die Entstehung von Spontanschmerzen. Im Vortrag von J. Tesarz soll daran anknüpfend auf entsprechende psychophysiologische Veränderungen bei Patienten mit nicht-spezifischem Rückenschmerz und frühen Stresserfahrungen eingegangen werden. Es soll dargestellt werden, dass bei chronischen Rückenschmerzpatienten unterschiedliche Arten frühkindlicher Stresserfahrungen mit spezifischen Veränderungen im somatosensorischen System einhergehen. So geht in Hinsicht auf verschiedene Unterarten früherer Stressbelastungen emotionaler Missbrauch mit einer gesteigerten spinalen Sensibilisierung einher, während sexueller Missbrauch mit einer erhöhten Berührungsempfindlichkeit assoziiert ist. Diese Ergebnisse legen nahe, dass frühere Stresserfahrungen mit nachhaltigen Veränderungen in der somatosensorischen Funktion assoziiert sind. Abschließend sollen im Vortrag von M. Noll-Hussong im Überblick Bildgebungsbefunde zum Zusammenhang zwischen traumatisierenden Stresserfahrungen und der Ausbildung chronischer Schmerzen bei Patienten mit multisomatoformen Schmerzstörung dargestellt werden. So wurde z.B. mittels eines Empathie-Paradigmas mit Hilfe der fMRI die zentralnervöse Schmerzverarbeitung bei traumatisierten im Vergleich zu nicht traumatisierten Patienten mit einer chronischen Schmerzstörung untersucht. Hierbei zeigte sich eine veränderte neuronale Prozessierung von empathisch wahrgenommenem Schmerz zwischen missbrauchten und nicht missbrauchten Patienten u. a. in limbischen Arealen. Das Symposium schließt mit einer Diskussion über mögliche Implikationen für die Behandlung.

Freitag, 21.10.2016

\section{Schmerz bei Kindern und im Alter}

\section{SY14 - Zeit zum (Um)denken bei älteren Patienten mit Rückenschmerzen}

\section{P. Nilges' ${ }^{1}$ M. Laekeman ${ }^{2}$, D. Seeger ${ }^{3}$}

'Johannes Gutenberg-Universität, Psychologisches Institut, Mainz, Deutschland, ${ }^{2}$ Universität Witten/Herdecke (PhD Studentin), Fakultät für Gesundheit, Witten, Deutschland, ${ }^{3}$ Universitätsmedizin Göttingen; Georg-August-Universität, Schmerzklinik/BE Physiotherapie, Göttingen, Deutschland

1. „Das können Sie in Ihrem Alter vergessen“: Einfluss von BehandlerEinstellungen auf Schmerzen älterer Patienten. Paul Nilges, Mainz

2. Einstellungen junger Therapeuten in Bezug auf ältere Patienten. Marjan Laekeman, Bochum; Katrin Kuss, Marburg

3. Bewegen zum Erhalt der Bewegungssicherheit. Dagmar Seeger, Göttingen

Chronische Rückenschmerzen führen bei älteren Menschen oft zu Funktionseinbußen bis hin zum Verlust von Autonomie und Lebensqualität. Daher nehmen aktivierende Maßnahmen eine wichtige Rolle für das Schmerzmanagement dieser Population ein. Studien an jüngeren Patienten (18-65 J.) verdeutlichen die Bedeutung von Behandler-Einstellungen für eine leitliniengerechte aktivierende Therapie. Bewegungsängstliche Einstellungen des Therapeuten bezüglich Aktivitäten von Rückenschmerzpatienten können die Bewegungsängste des Patienten verstärken [1], für ältere Patienten wurde dies allerdings bisher kaum untersucht [2]. P. Nilges verdeutlicht an Hand einer Literaturübersicht den Einfluss von BehandlerEinstellungen auf Einstellungen und Verhalten, Befinden und Aktivitätsniveau bei älteren Menschen mit Rückenschmerzen. Konzepte, bei denen Degeneration als „typischer" und unvermeidbar mit dem Alterungsprozess verbundener Vorgang verstanden wird, führen vor allem bei älteren
Patienten zu Resignation und ungünstigen Bewältigungsstrategien. Übertriebene "Aktivierung" und „Zweckoptimismus" als Gegenposition werden der Situation und den Zielen älterer Patienten ebenso wenig gerecht. Bestehende Grenzen respektvoll zu explorieren und gemeinsam realistische Ziele und Schritte zu entwickeln, sind eine besondere Herausforderung für diese Patientengruppe. M. Laekeman und K. Kuss untersuchten die Einstellung von Studierenden der Gesundheitsberufe bezüglich Rückenschmerzen speziell bei älteren Menschen. In einer Querschnittstudie wurden die Studierenden ( $n=34)$ befragt, um ihre Aktivitätsempfehlungen für jüngere vs. ältere Patienten zu evaluieren. Es wurden zwei identische Fallvignetten benutzt, wobei für ein Teil der Gruppe das Alter der Patientin mit 40 Jahren angegeben wurde, bei dem anderen Teil mit $70 \mathrm{~J}$. Die Studierenden gaben Empfehlungen bzgl. Arbeit, Training, Alltagsaktivitäten und Bettruhe ab. Es gab eine Tendenz zur weniger angemessenen Empfehlungen bei älteren Menschen und $50 \%$ der Studierenden hielt weitere medizinische Diagnostik (z. B. MRT) für angebracht. D. Seeger beschreibt ihre Beobachtung einer Gruppe von älteren Schmerzpatienten, die regelmäßig aktiv blieben und somit sekundärpräventiv profitieren konnten. Dabei wird der aktuelle Gesundheitszustand in Bezug auf die vorangegangenen Diagnosen und den aktuellen Leistungsstand gesetzt. Kleine Videosequenzen geben einen Einblick in die Bewegungssicherheit einzelner, zwischen 70 und 80-jährigen Teilnehmer einer Trainingsgruppe.

\section{Literatur}

1. Darlow B, Fullen BM, Dean S, Hurley DA, Baxter GD, Dowell A (2012) The association between health care professional attitudes and beliefs and the attitudes and beliefs, clinical management, and outcomes of patients with low back pain: a systematic review. Eur J Pain 16(1):3-17.

2. Laekeman M, Leonhardt C (2015) Einstellungen und Überzeugungen von Behandlern bei älteren Patienten mit Rückenschmerzen. Eine Analyse der Erhebungsinstrumente und Forschungslücken. Schmerz 29(4):362-70.

\section{Epidemiologie und Genetik}

\section{SY15 - Genom und Proteom in Schmerzpathophysiologie - Welche Möglichkeiten bieten neue Analysemethoden}

\section{Segelcke ${ }^{1}$, M. Schmidt ${ }^{2}$, E. Niederberger ${ }^{3}$, E. Pogatzki-Zahn ${ }^{1}$}

'Universitätsklinikum Münster, Klinik für Anästhesiologie, postoperative Intensivmedizin und Schmerztherapie, Münster, Deutschland, ${ }^{2}$ Max Planck Institute of Experimental Medicine, Göttingen, Deutschland, ${ }^{3}$ pharmazentrum frankfurt/ZAFES, Institut für Klinische Pharmakologie, Frankfurt, Deutschland

1. Epigenetik in der Schmerzforschung-Welche Erkenntnisse gibt es aus der Grundlagenforschung? - Ellen Niederberger, Frankfurt

2. Epigenetik und Schmerz: Gibt es eine klinische Relevanz? - Daniel Segelcke, Münster

3. Neue Tools in der Schmerzforschung-Welche Chancen bietet uns Proteomics? - Manuela Schmidt, Göttingen

Die Therapie chronischer Schmerzen ist immer noch nicht zufriedenstellend möglich. Interessant sind in diesem Zusammenhang u. a. interindividuelle Unterschiede im Auftreten von Schmerzen und der Chronifizierung von Schmerz, so wie eine deutliche unterschiedliche interindividuelle Effektivität therapeutischer Substanzen, auch bei gleichen Schmerzentitäten. Teilweise erklärbar ist dies möglicherweise durch die erhebliche Variationsbreite des menschlichen Erbgutes, welches in der modernen Schmerztherapie bisher weitestgehend unberücksichtigt bleibt. Durch beträchtliche Fortschritte im Bereich von Genom- und Proteom-Analysen können Prozesse, welche zu einer Schmerzentstehung und -chronifizierung beitragen, im großen Maßstab untersucht werden. Hierbei konnte in den letzten Jahren gezeigt werden, dass epigenetische Prozesse, wie DNA-Methylierung oder Histonmodifikation, und die daraus resultierenden Veränderungen im Proteom für das Schmerzgeschehen eine wichtige Rolle spielen. Untersuchungen auf diesem Gebiet ermöglichen das Identifizieren neuer Angriffspunkte für moderne Therapieansätze in der Schmerzforschung. 
In diesem Symposium sollen Schwerpunktmäßig Grundlagen zur Genom- und Proteomforschung im Zusammenhang mit Schmerz ebenso wie ganz aktuelle Erkenntnisse aus dieser Forschung im thematischen $\mathrm{Zu}$ sammenhang vermittelt werden. Es soll die Frage diskutiert werden, welche klinische Relevanz epigenetische Modifikationen haben und inwieweit die moderne Proteomforschung für neue Therapieansätze nutzbar gemacht werden kann. Aufgrund des breit angelegten Themenbereiches richtet sich das Symposium sowohl an Kliniker, als auch an Grundlagenwissenschaftler, die sich näher mit der Rolle von genomischen und proteomischen Analysen und derer Möglichkeiten für die moderne Schmerzforschung beschäftigen wollen.

Frau PD Dr. Ellen Niederberger wird einen Überblick über Grundlagen der Epigenetik geben und ihren Schwerpunkt vor allem auf die Forschungsergebnisse aus tierexperimentellen Arbeiten legen und aufzeigen, welche epigenetischen Modifikationen für die Schmerzpathophysiologie eine Rolle spielen könnten. Herr Dr. Daniel Segelcke wird in seinem Vortrag aktuelle Ergebnisse aus der tierexperimentellen Grundlagenforschung dahingehen analysieren, welche Relevanz diese für den klinischen Alltag haben. Er wird diskutieren, inwiefern die erlangten Erkenntnisse auf dem Gebiet der Epigenetik zu neuen Therapieansätzen führen können. Der Vortrag von Frau Dr. Manuela Schmidt wird sich mit modernen Proteom-Analysen in der Schmerzpathophysiologie beschäftigen und aufzeigen, welche Chancen diese Methoden für die Schmerzforschung bietet.

\section{Neurologie}

\section{SY16 - Update Kopfschmerz}

\section{S. Förderreuther', U. Reuter ${ }^{2}$, D. Holle-Lee}

${ }^{1}$ Neurologische Klinik, LMU, Konsildienst, München, Deutschland, ${ }^{2}$ Charité Universitätsmedizin Berlin, Neurologische Klinik und Poliklinik, Berlin, Deutschland, ${ }^{3}$ Universitätsklinikum Essen (AöR), Neurologische Klinik/ Westdeutsches Kopfschmerzzentrum, Essen, Deutschland

Durch das bessere Verständnis der Pathophysiologie primärer Kopfschmerzerkrankungen, insbesondere der Migräne und des Cluster Kopfschmerzes wurden in der Vergangenheit bereits gezielt neue Substanzklassen wie beispielsweise die Triptane entwickelt und mit Erfolg in die Kopfschmerztherapie eingeführt. Andere vielversprechende Therapieansätze wie z. B. die Entwicklung von Substanz P Antagonisten waren dagegen nicht erfolgreich, ohne dass man dies mit den etablierten pathophysiologischen Kopfschmerzmodellen erklären könnte. An Beispiel der Migräne mit ihren vielen klinischen Facetten kann man erahnen, wie komplex und vielschichtig deren Pathophysiolgie ist: Verschiedene neuronale Systeme zeigen eine Dysfunktion. Es gibt hierfür unterschiedliche genetische Prädispositionen und bildgebende Daten, die belegen, dass ein Migräne-Gehirn strukturell und funktionell verändert ist. In der Summe resultiert hieraus eine generalisierte neuronalen Hyperexcitbilität, die letztlich zu einer neuro-vaskulären Entzündung führt.

Bei der Entwicklung neuer Therapieansätze stehen derzeit bei Migräne und Clusterkopfschmerz CGRP und das parasympathische Nervensystem (N. vagus, Ggl. sphenopalatinum) im Fokus. Sie stellen Bausteine in der Kopfschmerzpathophysiologie dar, die durch Antikörper bzw. Stimulationsverfahren beeinflusst werden können. Sie haben so bereits ihren Weg aus der Grundlagenforschung in die klinische Kopfschmerztherapie genommen. In dem Symposium soll die Brücke von den aktuellen Erkenntnissen der Kopfschmerzpathophysiologie hin zu den neuen Therapieformen geschlagen werden. Auch wenn Migräne und Clusterkopfschmerz einen Schwerpunkt bilden werden, so sollen auch die pathophysiologischen Mechanismen, die zu Spannungskopfschmerzen und Schmerzmittel-induzierten Kopfschmerzen führen, vorgestellt werden, um auch hier mögliche Therapieoptionen zu diskutieren. Für die Kliniker besonders interessant: die neuesten Daten ud Erfahrungen zu den CGRP(-Rezeptor)Antikörpern und zu den diversen heute bereits für die Patienten zugänglichen Stimulationsverfahren.

\section{Schmerz nach Operationen}

\section{SY17 - Hypnose in der Grundlagenforschung und in der Therapie akuter und chronischer Schmerzen}

\section{Faymonville' ${ }^{1}$ E. Hansen², W. Häuser ${ }^{3}$}

'Universitätskrankenhaus Lüttich, Schmerzklinik, Lüttich, Belgien, ${ }^{2}$ Universitätsklinikum Regensburg, Klinik für Anästhesiologie, Regensburg, Deutschland, ${ }^{3}$ Klinikum Saarbrücken, Klinik Innere Medizin 1, Saarbrücken, Deutschland

Hypnose ist das älteste nicht-medikamentöse Heilverfahren zur Behandlung akuter und chronischer Schmerzen. In dem Symposium sollen sowohl theoretisches als auch praktisches Wissen vermittelt werden. Ein Schwerpunkt des Symposiums ist die Darstellung aktueller Studienergebnisse der experimentellen Hypnose in der Grundlagenforschung akuter und chronischer Schmerzen. Weitere Schwerpunkte sind die Zusammenfassung aktueller systematischer Übersichtsarbeiten mit Meta-analysen zur Wirksamkeit und Sicherheit von Hypnose in der Schmerzmedizin und Beispiele für die Verwendung hypnotischer Techniken im medizinischen Alltag. Frau Professor Faymonville hat seit 1992 in der Universitätsklinik in Lüttich (Belgien) die neue Anästhesietechnik „Hypnosedation“ bei mehr als 8000 chirurgischen Patienten mit Erfolg angewandt. Diese Technik kombiniert Hypnose mit einer sehr leichten Wachsedierung und Lokalanästhesie. In retrospektiven und prospektiven klinischen Studien wurde festgestellt, dass die Hypnosetechnik den Komfort der Patienten während und nach der Chirurgie erhöht. Neurophysiologische Studien mit PET Scanner Technik und funktionelle MRI ermöglichen es uns jetzt, die Schmerzmodulation durch Hypnose besser zu verstehen.

Herr Professor Hansen wird aktuelle systematische Übersichtsarbeiten mit Meta-analysen zur Wirksamkeit der prä-, intra-, und postoperativen Hypnose bei operativen Eingriffen darstellen. Die Möglichkeiten von Hypnose bei Wachkraniotomien werden an Hand eines Videos vorgestellt.

Herr PD Dr. Häuser wird aktuelle systematische Übersichtsarbeiten mit Meta-analysen zur Wirksamkeit der Hypnose bei chronischen Schmerzsyndromen (z. B. Reizdarmsyndrom, Fibromyalgiesyndrom; Kopfschmerzen) vorstellen und ihren Stellenwert innerhalb eines multimodalen Therapiekonzeptes diskutieren. Die Bauchhypnose bei Reizdarmsyndrom wird an Hand eines Fallbeispieles vorgestellt.

Herr Professor Hansen und Herr PD Dr. Häuser werden die Chancen und Risiken der Kommunikation von Ärzten und Pflegepersonal (sogenannte Wachsuggestionen) mit Patienten in medizinischen Alltag (z. B. Aufklärungsgespräch; Vorbereitung auf und Durchführung von medizinischen Eingriffen wie Blutentnahmen, Endoskopien und Operationen) darstellen und die Verbindungen zur Placebo- und Noceboforschung diskutieren.

\section{Varia}

\section{SY18 - Qualitätssicherung und Zertifizierung in der Multimodalen Schmerztherapie}

M. Pfingsten', U. Marschall' ${ }^{2}$ R. Sabatowski ${ }^{3}$, B. Nagel ${ }^{4}$

'Universitätsmedizin Göttingen, Schmerzklinik, Göttingen, Deutschland, 'BARMER GEK, Kompetenzzentrum Medizin, Wuppertal, Deutschland, "UniversitätsSchmerzCentrum, Universitätsklinikum "Carl Gustav Carus", Dresden, Deutschland, ${ }^{4}$ DRK Schmerz-Zentrum Mainz, Tagesklinik für interdisziplinäre Schmerztherapie, Mainz, Deutschland

1. Entwicklung der MMST aus Kostenträger-Sicht (Häufigkeiten - Pat.Merkmale - Effizienz); U. Marschall

2. Besonderheiten und Probleme der MMST in der Krankenhausbehandlung (OPS); R. Sabatowski

3. Definition von Qualitätsmerkmalen der MMST im kurativen Bereich; B. Nagel

Multimodale Schmerztherapie wird in D - insbesondere im Rahmen von Kollektivverträgen (OPS) zunehmend häufiger angewendet. So stieg die Zahl 
der „multimodal“ arbeitenden Einrichtungen laut Barmer GEK Krankenhausreport 2015 von 2006 bis 2014 um 368\%. Die Zahl der Einrichtungen, die im Jahr 2013 den OPS-Code 8-918 (stationäre multimodale Schmerztherapie abrechneten lag bei 427. Die Effizienz dieser zunehmend gegenüber den Krankenkassen geltend gemachten Behandlung wird in letzter Zeit nur noch dann sichtbar, wenn besondere Pat.-Merkmale wie z. B. die hohe KoMorbiditätslast berücksichtigt werden. Bei der Betrachtung der verfügbaren Zahlen fällt auf, dass die Behandlungszahlen einer großen Variabilität unterliegen und es ist z. B. für die auffallend hohe Zahl an Krankenhäusern, die nur vereinzelt den OPS-Code 8-918 zur Abrechnung bringen, kaum vorstellbar, dass die von der Adhoc-Kommission „Multimodale Schmerztherapie“ geforderten strukturellen und auch inhaltlichen Vorgaben, die weitaus präziser sind als vom OPS-Code vorgegeben, auch nur annähernd erfüllt sind. Auch die Verweildauer, wie sie im Bericht der Barmer GEK dargestellt wird, zeigt einer möglicherweise problematischen Tendenz auf, die wiederum den Empfehlungen zur Therapieintensität von $>100$ Stunden widerspricht.

Diese Problematik und mögliche Auswege hieraus werden in dem Symposium aus verschiedenen Perspektiven diskutiert. So werden die aktuellen Krankenkassendaten und die daraus gefolgerten Schlüsse der Barmer GEK vorgestellt (Marschall). Grundlegende Probleme erwachsen möglicherweise im Kontext der Rahmenbedingungen, die aus den abgeschlossenen Verträgen (z. B. Kollektivvertrag vs. Selektivvertrag) resultieren, vor allem wenn die Durchführungen der multimodalen Schmerztherapie den grundlegenden Prinzipien z. T. sogar widersprechen (Sabatowski). Um die Qualität multimodaler Schmerztherapie zu sichern, hat eine Kommission der Dt. Schmerzgesellschaft im Jahr 2016 nachprüfbare Qualitätskriterien für die Struktur-, Prozess und Ergebnisqualität definiert, die aktuell als Grundlage für eine zukünftige Zertifizierung multimodaler Schmerztherapie dienen können (Pfingsten).

\section{Neurologie}

\section{SY19 - Stratifizierung von Patienten mit Nervenschmerzen: kommt die mechanismenbasierte Therapie?}

\section{W. Magerl', R. Baron'², C. Maihöfner ${ }^{3}$}

'Medizinische Fakultät Mannheim, Lehrstuhl für Neurophysiologie, CBTM, Mannheim, Deutschland, ${ }^{2}$ Klinik für Neurologie, Universitätsklinikum Schleswig-Holstein, Campus Kiel, Sektion Neurologische Schmerzforschung und -therapie, Kiel, Deutschland, ${ }^{3}$ Klinikum Fürth, Chefarzt der Neurologischen Klinik, Fürth, Deutschland

1. Ralf Baron (Kiel) - Clusteranalyse von Netzwerkdaten neuropathischer Schmerzpatienten (DFNS und IMI-Neuropain)

2. Walter Magerl (Mannheim) - Biomarker und Stratifizierungsstrategien

3. Christian Maihöfner (Fürth) - Responder-Muster der Behandlung (QUEPP, ELEVATE und PEPPERs)

Die Therapie neuropathischer Schmerzen hat sich in der Vergangenheit als notorisch problematisch erwiesen und der Behandlungserfolg mit jeglicher Klasse von Pharmaka als limitiert. Das vorgeschlagene Symposium zielt auf die Darstellung von Faktoren, die die Grundlage dieser begrenzten Wirksamkeit sind. Es hat zum Ziel darzustellen, dass die Heterogenität von Patienten mit neuropathischem Schmerz ihren Hintergrund hat im variablen Beitrag unterschiedlicher Pathomechanismen des neuropathischen Schmerzes, die unterschiedliche Strategien der Behandlung erfordern. Die einzelnen Beiträge des Symposiums sollen zeigen, dass es gelingt mit Hilfe einer im vergangenen Jahrzehnt aus dem Deutschen Forschungsnetz neuropathischer Schmerz (DFNS) entwickelten Methodik der standardisierten Erhebung sensorischer Veränderungen (Quantitative Sensorische Testung QST) unterschiedliche Typen von Patienten durch Clusteranalyse zu identifizieren, denen tentativ wiederum unterschiedliche Mechanismen der Pathologie zugeordnet werden können (Vortrag Ralf Baron / Kiel). Diese Stratifikation konnte in großen Patientenkohorten $(n>1000)$ als verlässlich identifiziert werden und hat sich in ersten Behandlungsstudien bereits als rationale Grundlage zur Vorhersage eines differentiellen Behandlungs- erfolgs bewährt (Demant et al. PAIN 2014). Sie ist von der European Medicines Agency (EMA) für pharmakologische Studien als explorative Strategie zur Klassifizierung von Patienten akzeptiert (EMA/CHMP/970057/2011). Im zweiten Vortrag (Walter Magerl / Mannheim) soll auf die Identifizierung biologischer Marker (Signalmoleküle, Rezeptoren, genetische Marker) eingegangen werden (Hayes et al. Current Opinion Pharmacol 2014, Yaksh et al. F1000 Reports 2015) und demonstriert werden, dass sich Kandidaten aus dem Repertoire der verfügbaren Biomarker mit der Stratifizierungsstrategie des DFNS in Übereinstimmung bringen lassen. Es soll dabei insbesondere anhand einiger Beispiele auf genetische Marker eingegangen werden (z.B. Tegeder et. al. Nature Med 2006, Black et al. Ann Neurol 2008, Binder et al. PLoS ONE 2011, Faber et al. Neurology 2012, Latremoliere et al. Neuron 2015).

Im dritten Vortrag (Christian Maihöfner / Fürth) soll gezeigt werde, dass bei der Behandlung des neuropathischen Schmerzes Responderprofile identifiziert werden können. Es sollen dabei Daten zu Behandlungen mit peripheren (hochdosiertes Capsaicin) und zentralnervösen Angriffspunkten (Pregabalin) aus den klinischen Studienprogrammen QUEPP, ELEVATE und PEPPERS dargestellt werden. Es soll dabei gezeigt werden, dass auch Responder und Non-Responder sich auf der Grundlage der Stratifierung des neuropathischen Schmerzes verstehen lassen (Martini et al. Eur J Pain 2013, Maihöfner und Heskamp Curr Med Res Opin 2013, Eur J Pain 2014, Höper et al. Curr Med Res Opin 2014).

\section{Literatur}

1. Binder A, May D, Baron R, Maier C, Tölle TR, Treede RD, Berthele A, Faltraco F, Flor H, Gierthmühlen J, Haenisch S, Huge V, Magerl W, Maihöfner C, Richter H, Rolke R, Scherens A, Uçeyler N, Ufer M, Wasner G, Zhu J, Cascorbi I (2011) Transient receptor potential channel polymorphisms are associated with the somatosensory function in neuropathic pain patients. PLoS One 6:e17387.

2. Black JA, Nikolajsen L, Kroner K, Jensen TS, Waxman SG (2008) Multiple sodium channel isoforms and mitogen-activated protein kinases are present in painful human neuromas. Ann Neurol 64:644-653.

3. Demant DT, Lund K, Vollert J, Maier C, Segerdahl M, Finnerup NB, Jensen TS, Sindrup SH (2014) The effect of oxcarbazepine in peripheral neuropathic pain depends on pain phenotype: a randomised, double-blind, placebo-controlled phenotypestratified study. Pain 155:2263-2273.

4. Faber CG, Hoeijmakers JG, Ahn HS, Cheng X, Han C, Choi JS, Estacion M, Lauria G, Vanhoutte EK, Gerrits MM, Dib-Hajj S, Drenth JP, Waxman SG, Merkies IS (2012) Gain of function Nav1.7 mutations in idiopathic small fiber neuropathy. Ann Neurol 71:26-39.

5. Hayes AG, Arendt-Nielsen L, Tate S (2014) Multiple mechanisms have been tested in pain--how can we improve the chances of success? Curr Opin Pharmacol. 14:11-17.

6. Höper J, Helfert S, Heskamp ML, Maihöfner CG, Baron R (2014) High concentration capsaicin for treatment of peripheral neuropathic pain: effect on somatosensory symptoms and identification of treatment responders. Curr Med Res Opin. 30:565-574.

7. Latremoliere A, Latini A, Andrews N, Cronin SJ, Fujita M, Gorska K, Hovius R, Romero C, Chuaiphichai S, Painter M, Miracca G, Babaniyi O, Remor AP, Duong K, Riva P, Barrett LB, Ferreirós N, Naylor A, Penninger JM, Tegeder I, Zhong J, Blagg J, Channon KM, Johnsson K, Costigan M, Woolf CJ (2015) Reduction of Neuropathic and Inflammatory Pain through Inhibition of the Tetrahydrobiopterin Pathway. Neuron 86:1393-1406.

8. Maihofner C, Heskamp ML (2013) Prospective, non-interventional study on the tolerability and analgesic effectiveness over 12 weeks after a single application of capsaicin 8\% cutaneous patch in 1044 patients with peripheral neuropathic pain: first results of the QUEPP study. Curr Med Res Opin 29:673-683.

9. Maihöfner CG, Heskamp ML (2014) Treatment of peripheral neuropathic pain by topical capsaicin: Impact of pre-existing pain in the QUEPP-study. Eur J Pain 18:671-679.

10. Martini CH, Yassen A, Krebs-Brown A, Passier P, Stoker M, Olofsen E, Dahan A (2013) A novel approach to identify responder subgroups and predictors of response to low- and high-dose capsaicin patches in postherpetic neuralgia. Eur J Pain. 17:1491-1501.

11. Tegeder I, Costigan M, Griffin RS, Abele A, Belfer I, Schmidt H, Ehnert C, Nejim J, Marian C, Scholz J, Wu T, Allchorne A, Diatchenko L, Binshtok AM, Goldman D, Adolph J, Sama S, Atlas SJ, Carlezon WA, Parsegian A, Lötsch J, Fillingim RB, Maixner W, Geisslinger G, Max MB, Woolf CJ (2006) GTP cyclohydrolase and tetrahydrobiopterin regulate pain sensitivity and persistence. Nat Med 12:1269-1277.

12. Yaksh TL, Woller SA, Ramachandran R, Sorkin LS (2015) The search for novel analgesics: targets and mechanisms. F1000Prime Rep 7:56. 


\section{Individualisierte Schmerztherapie}

\section{SY20 - Was zählt, ist normales Leben, nicht Kosmetik: Schmerztherapeutische Langzeitverläufe!}

\section{Maier ${ }^{1}$, A. Diezemann'2 J. Wager ${ }^{3}$}

'Berufsgenossenschaftliches Universitätsklinikum Bergmannsheil GmbH, Bochum, Abteilung für Schmerzmedizin, Bochum, Deutschland, ${ }^{2} \mathrm{DRK}-$ Schmerz-Zentrum Mainz, Tagesklinik für interdisziplinäre Schmerztherapie, Mainz, Deutschland, ${ }^{3}$ Vestische Kinder- und Jugendklinik Datteln, Deutsches Kinderschmerzzentrum, Datteln, Deutschland

1. Prototypische Langzeitverläufe invasiver Schmerztherapie, Prof. Dr. Christoph Maier, Bochum

2. Langzeitergebnisse nach multimodaler teilstationärer Schmerztherapie im Erwachsenenalter, Dr. Dipl.-Psych. Anke Diezemann, Mainz

3. Langzeitergebnisse nach multimodaler Schmerztherapie im Kindesund Jungendalter, Dr. rer.-nat. Dipl.-Pych. J. Wager, Datteln

In klinischen Studien und Vorträgen werden zumeist die kurzfristigen Ergebnisse einer Schmerztherapie untersucht und berichtet. Die Untersuchung der Langzeitverläufe ist mit logistischen Schwierigkeiten behaftet und kostspielig. Es besteht zudem für den Forscher und Kliniker die Gefahr, dass sich Kurzzeiteffekte nicht reproduzieren lassen. Aus Patientensicht jedoch und im Interesse der Gesellschaft ist nicht (nur) die kurzzeitige Reduktion der Schmerzwerte, sondern die langfristig stabile $\mathrm{Zu}$ nahme an Funktion bei akzeptablem Schmerzniveau im Anschluss an eine Schmerztherapie relevant.

Christoph Maier fokussiert in seinem Vortrag prototypische Langzeitverläufe nach invasiver Schmerztherapie chronischer Schmerzen. Es werden Kasuistiken präsentiert typischer Langzeitverläufe nach Spinal Cord Stimulation (SCS) bei CRPS sowie der intrathekalen Opioid- und Ziconitidtherapie bei Tumor- und Nicht-Tumorschmerzen. Anke Diezemann beschäftigt sich mit den Langzeitergebnissen nach multimodaler (teilstationärer) Schmerzbehandlung Erwachsener. Sie wird die unterschiedlichen am Schmerzsyndrom orientierten Behandlungsschwerpunkte bei Patienten-Gruppen mit Nackenschmerz, Rückenschmerz, Multilokulären Beschwerden und Kopfschmerzen darstellen. Die Ergebnisse nach 12 Monaten hinsichtlich schmerzbezogener Daten, Lebensqualität, Zufriedenheit mit der Therapie und auch Inanspruchnahme des Gesundheitswesens in Anhängigkeit vom Schmerzsyndrom, Chronifizierungsstadium und bestehenden Komorbiditäten werden beschrieben und diskutiert. In dem abschließenden Vortrag widmet sich Julia Wager der langfristigen Wirksamkeit schmerztherapeutischer Verfahren bei chronisch schmerzkranken Kindern und Jugendlichen. Eine Literaturübersicht und ein vier Jahres Follow-up einer stationären Schmerztherapie werden berichtet. Hierbe werden nicht nur Wirksamkeitsdaten auf den Ebenen Schmerz, emotionale Beeinträchtigung und Alltagsfunktion betrachtet, sondern auch ökonomische Aspekte sowie die Interaktion zwischen erfolgreicher Schmerztherapie und Ökonomie.

\section{Neurologie}

\section{SY21 - Müssen wir bei der Zentralen Sensiblisierung umdenken?}

\author{
J. Sandkühler', M. Finnern'², S. Rehm ${ }^{3}$
}

'Zentrum für Hirnforschung, Medizinische Universität Wien, Wien, Österreich, ${ }^{2}$ Central Institute of Mental Health, University of Heidelberg, Mannheim, Department of Cognitive and Clinical Neuroscience, Mannheim, Deutschland, ${ }^{3}$ Klinik für Neurologie, UK-SH, Campus Kiel, Sektion für Neurologische Schmerzforschung und Therapie, Kiel, Deutschland
Translationale Schmerzforschung (Topic)

1. Was versteht man unter „Zentraler Sensiblisierung“? (Prof. Dr. J. Sandkühler, Wien)

2. Was messen unsere globalen Chronizitätsindizes? (M. Finnern, Mannheim)

3. Was führt beim Menschen zu zentralen Sensiblisierungsvorgängen? (Dr. S. Rehm, Kiel)

Der Begriff „Zentrale Sensibilisierung“ wird in der Schmerzmedizin und der Schmerzforschung häufig gebraucht, allerdings in unterschiedlichen, sich teils widersprechenden Definitionen [1]. Laut IASP ist damit eine Übererregbarkeit von nozizeptiven Neuronen im zentralen Nervensystem gemeint. Andere Autoren verwenden ihn als Sammelbegriff für alle zentralnervösen Mechanismen der Hyperalgesie und Allodynie. Im ersten Teil des Symposiums sollen zunächst die unterschiedlichen Konzepte zur "Zentralen Sensibilisierung" vorgestellt werden und deren Bedeutung in der experimentellen Schmerzforschung kritisch diskutiert werden. Der zweite Teil wird sich mit Untersuchungen zur Messung veränderter Schmerzsensibilität mit psychophysikalischen Methoden und deren Beeinflussungsfaktoren sowie deren Zusammenhang mit globalen Chronizitätsindizes befassen.

Abschließend soll diskutiert werden welche Implikationen sich aus dem derzeitigen Wissensstand für zukünftige Forschungsprojekte ergeben. Aufgrund der Möglichkeit diese Thematik aus unterschiedlichsten Perspektiven (physiologisch, klinisch und psychologisch) beleuchten zu können, richtet sich dieses Symposium sowohl an Wissenschaftler als auch an klinisch tätige Schmerztherapeuten.

Herr Prof. Sandkühler wird zunächst mit einer kritischen Einleitung in das Thema beginnen und dabei einerseits aktuelle tierexperimentelle Daten vorstellen, andererseits auch den Bogen zur translationalen Forschung schlagen und herausarbeiten welche Sensibilisierungsvorgänge eine besondere klinische Relevanz haben. Im zweiten Vortrag wird Frau Finnern Ergebnisse zum Thema veränderte Schmerzsensibilität und Dimensionsstruktur globaler Chronizitätsindizes vorstellen. Dabei wurden die Relation von Dauer und Schwere der Schmerzsymptomatik, die Dimensionalität der Chronizitätsscores und deren Zusammenhänge mit einer veränderten Schmerzverarbeitung untersucht $[2,3]$. Besonders interessant ist die Frage inwieweit unsere derzeit eingesetzten Chronizitätsscores tatsächlich klinisch relevante zentrale Umbauvorgänge erfassen können. Im dritten Vortrag wird Frau Dr. Rehm aktuelle Daten von Untersuchungen mittels quantitativer sensorischer Testung an Patienten mit chronischen Schmerzsyndromen (Osteoarthrose, Fibromyalgiesyndrom und Rückenschmerzen) darstellen. Dabei soll gezeigt werden, welche Paramater (z. B. Schmerzintensität, Schmerzdauer, psychiatrische Komorbiditäten) bei unseren Patienten Einfluss auf die klinisch messbaren Parameter der zentralen Sensibilisierung nehmen könnten.

\section{Literatur}

1. siehe Physiological Reviews 89 (2009) 707 und Texbook of Pain, 2013, Kapitel 6.

2. Finnern M, Hölzl R, Kleinböhl D, Benrath J (2015) Complexities of pain chronicity: Coding intensity and duration of musculoskeletal pain on Axis IV of the IASP Taxonomy. Schmerz 29(Suppl. 2):1-S87. doi: 10.1007/s00482-015-0067-4

3. Kleinbohl D, Hölzl R, Moltner A, Rommel C, Weber C, Osswald PM (1999). Psychophysical measures of sensitization to tonic heat discriminate chronic pain patients. Pain 81:35-43. 


\section{Neurologie}

\section{SY23 - DMKG meets HNO - relevante Schmerzsyndrome von Hals, Nase und Ohr}

\section{K. Meßlinger ${ }^{1}$, K. Rak², T. Jürgens ${ }^{3}$}

'Universität Erlangen-Nürnberg, Institut für Physiologie \& Pathophysiologie, Erlangen, Deutschland, ${ }^{2}$ Universitätsklinikum Würzburg, Klinik und Poliklinik für Hals-, Nasen- und Ohrenkrankheiten, Würzburg, Deutschland ${ }^{3}$ Universitätsmedizin Rostock, Klinik und Poliklinik für Neurologie, Rostock, Deutschland

1. Vortrag: Prof. Dr. Karl Messlinger (Universität Erlangen): „Warum es weh tut - Schmerzphysiologie von Hals, Nase und Ohr"

2. Vortrag: Dr. Kristen Rak (Universität Würzburg): „Relevante Schmerzsyndrome in der HNO"

3. Vortrag: PD Dr. Tim Jürgens (Rostock): „Neurologische Schmerzsyndrome von Hals, Nase und Ohren" (Chair)

Ziel dieses Symposiums ist es, für den Schmerztherapeuten relevante Erkrankungen im Bereich von Hals, Nase und Ohr sowohl aus Sicht des HNO-Arztes als auch des Neurologen vorzustellen und die entsprechenden anatomisch-physiologischen Grundlagen zu diskutieren.

Der erste Vortrag gibt eine Übersicht über die anatomischen und physiologischen Besonderheiten der Schmerzentstehung und -wahrnehmung von Hals, Nase und Ohr. Daneben werden schwerpunktmäßig eigene Daten zur nozizeptiven Innervation von Nasenschleimhaut und den Sinus vorgestellt.

Im zweiten Vortrag werden typische Schmerzsyndrome aus dem Alltag des HNO-Arztes präsentiert, die für den klinisch tätigen Schmerztherapeuten von Bedeutung sind. Hier wird ein weiter Bogen über entzündliche Erkrankungen (wie Sinusitis, Otitis und Parotitis), Tumoren bis hin zu rheumatologisch-autoimmunen Erkrankungen gespannt und ein Überblick über die aktuellen Therapieansätze gegeben.

Im letzten Vortrag wird auf wichtige neurologische Schmerzsyndrome mit Lokalisation in Hals, Nase und eingangen und Schwierigkeiten in deren Diagnostik und Therapie beleuchtet. Kontroverse Konzepte wie Kopfschmerzen durch „nasal contact points“ werden kritisch diskutiert.

\section{Schmerz nach Operationen}

\section{SY24 - Umdenken in der Akutschmerztherapie: Mythen zerplatzen lassen ... ?}

\section{F. Petzke', E. Pogatzki-Zahn², W. Meißner ${ }^{3}$}

'Georg-August-Universität Göttingen, ZARI, Schmerz-Tagesklinik und -Ambulanz, Göttingen, Deutschland, ${ }^{2}$ Universitätsklinikum Münster, Klinik für Anästhesiologie, postoperative Intensivmedizin und Schmerztherapie, Münster, Deutschland, ${ }^{3}$ Universitätsklinikum Jena, Abteilung $\mathrm{f}$. Palliativmedizin, Klinik f. Anästhesiologie u. Intensivtherapie, Jena, Deutschland

In der Akutschmerztherapie sind einige Vorgehensweisen so etabliert, dass man überhaupt nicht auf die Idee kommen würde, sie wirklich zu hinterfragen.

Opioide - Freund oder Feind: (Frank Petzke, Göttingen)

Opioide gelten als Grundpfeiler der pharmakologischen Akutschmerztherapie. Sie scheinen dann besonders ,effektiv“ zu sein, wenn vor allem eine möglichst ausgeprägte Reduktion der Schmerzintensität primäres Therapieziel ist. Dabei werden häufig die opioidinduzierten Probleme (PONV, Sedierung, GI-Effekte) ausgeblendet, die einer frühzeitigen postoperativen Erholung durchaus entgegenstehen können. Darüber hinaus mehren sich Hinweise, dass hochdosierte Opioide nicht nur akute Schmerzen verschlechtern, sondern auch chronische Schmerzen begünstigen könnten. Sollten wir nun auf intra- und postoperativ auf Opioide eher verzichten und welche alternativen Substanzen und Methoden können wir dann einsetzen?
QST perioperativ: Toll oder toy? (Esther Pogatzki-Zahn, Münster)

Es wäre klinisch hilfreich, wenn das Risiko für starke postoperative Akutschmerzen und Chronifizierung schon vor der Operation vorhersagbar wäre. Eine Methode, die zur Vorhersagbarkeit stark propagiert und in vielen klinischen Studien evaluiert worden ist, ist die QST. Der Einsatz von QST prä- und/oder früh postoperativ konnte - zumindest für einige QSTParameter - auch wirklich in einigen Studien zu einer relativ guten Vorhersagbarkeit beitragen. Allerdings sind die vielen negativen Studienergebnisse, die Variabilität des Erfolges (gerade für den chronischen postoperativen Schmerz) doch insgesamt eher ernüchternd. In diesem Beitrag sollen $\mathrm{Zu}$ sammenhänge zwischen QST-Befunden perioperativ und postoperativen akuten und chronischen Schmerzen kritisch beleuchtet, Gründe für eine geringe Prädiktion der meisten Parameter dargestellt und eine mögliche Bedeutung von QST in der Akutschmerztherapie diskutiert werden.

Schmerz als 5th Vitalzeichen: Alles ein großer Irrtum? (Winfried Meissner, Jena)

Eine möglichst häufige Messung der Schmerzintensität gilt als eine der wichtigsten Qualitätsindikatoren und Empfehlungen in Leitlinien und klinischen Handlungsempfehlungen weltweit. Es mehren sich jedoch die Hinweise, dass diese Empfehlung sowohl als Prozess als auch inhaltlich problematisch ist. Einerseits scheint eine häufige Schmerzmessung per se die Qualität nicht zu verbessern und hat möglicherweise sogar „Nebenwirkungen “, andererseits stellt sich die Frage, ob eine niedrige Schmerzintensität ein sinnvolles klinisches Behandlungsziel ist. In der chronischen Schmerztherapie deutet sich seit längerem eine Abkehr von diesem Outcome-Kriterium ab. Welche Perspektiven und Alternativen bieten sich in der Akutsachmerztherapie an? Eine an Funktionsparametern und Patientenpräferenzen orientierte Schmerztherapie würde ein Integral über Effektivität, aber auch unerwünschte Therapieeffekte darstellen und damit auch eine mögliche Übertherapie vermeiden.

\section{Neurologie}

\section{SY25 - Konzepte, praktische Umsetzung und Evidenzlage zur Edukation von (Kopf)Schmerzpatienten}

H. Nobis', M. von Wachter' ${ }^{2}$, C. Gaul ${ }^{3}$

'MEDIAN-Klinik am Burggraben, Orthopädische Psychosomatik/ Interdisziplinäre Schmerztherapie, Bad Salzuflen, Deutschland, ${ }^{2}$ OstalbKlinikum Aalen, Klinik für Psychosomatik, Aalen, Deutschland, ${ }^{3}$ Migräneund Kopfschmerzklinik, Königstein im Taunus, Deutschland

1. Dipl.-Psych. H-G Nobis: Edukation von Kopfschmerzpatienten - eine pädagogische Herausforderung?

2. Dr.med. M von Wachter: Interventionen und Instrumente der Edukation bei Kopfschmerz

3. Priv.-Doz. Dr. med. C Gaul: Was ist gesichert? - Evidenzlage der Edukation von Kopfschmerzpatienten

$93 \%$ der Patienten äußern den Wunsch nach umfassender u. verständlicher Information, jedoch kommen weniger als 30\% der Ärzte diesem Wunsch nach. Die IASP betont das ethische Recht der Patienten auf Information, im $\$ 2$ des Patientenrechtegesetzes wird der Behandler ,verpflichtet ....in verständlicher Weise ...sämtliche für die Behandlung wesentlichen Umstände zu erläutern". Edukation ist nicht nur Wunsch und Recht der Patienten, sondern ein wesentlicher Bestandteil einer erfolgreichen Schmerztherapie, da es so gelingen kann, Patienten zur Therapie zu motivieren und Compliance u. Adhärenz zu verbessern. „Vorurteile“ des Patienten, Schmerz sei ein lokales Geschehen, Schmerz weise immer auf einen körperlichen Defekt hin u. Skepsis gegenüber psycho-sozialen Mitwirkungsfaktoren können schnell zu Kommunikationsproblemen führen. Auf Therapeutenseite sind neben dem Verständnis des Bio-PsychoSozialen Schmerzmodells ein kopfschmerzspezifisches Krankheitsmodell zur Pathogenese u. Therapie, sowie grundlegende pädagogisch-methodische Kenntnisse u. Fertigkeiten erforderlich. Edukation kann helfen, die komplexen Zusammenhänge zwischen seelischen, körperlichen u. sozia- 
len Faktoren bei Kopfschmerz zu verstehen, Selbstwirksamkeit fördern u. passiv-regressiven Versorgungstendenzen entgegenwirken und schafft die Voraussetzung für eine erfolgreiche interdisziplinäre multimodale Therapie von Kopfschmerzerkrankungen. Durch eine umfassende Edukation kann das Einnahmeverhalten der Akutmedikation verbessert werden $u$. die Rezidivrate des Kopfschmerz durch Medikamentenübergebrauch reduziert werden.

Hans-Günter Nobis fokussiert auf die pädagogische Herausforderung einer patientengerechten Edukation unter kommunikativen Aspekten von Kopfschmerzpatienten. M von Wachter stellt typische Interventionstechniken (Schmerzbewältigungstraining, Umgang mit der Medikation, kognitive Umstrukturierung, Pausenmanagement u. a.) dar. Die Instrumente müssen an die Besonderheiten der gestörten Habituation von Migränepatienten und ihre dysfunktionalen Durchhaltestrategien angepasst werden. C. Gaulstellt Daten zur Wirksamkeit unterschiedlicher Edukationsstrategien und Ergebnisse multimodaler Therapieprogramme, bei denen Patientenedukation ein wesentlicher Bestandteil ist) vor.

\section{Literatur}

1. Nobis H, Pielsticker A (2013) Ärztliche Edukation und Kommunikation in der primären Schmerzbehandlung Klinische Relevanz und pädagogische Herausforderung. Schmerz 27:317-324.

2. Gaul C (2014) Kopfschmerzen - Patientenschulung als Baustein multimodaler Therapieprogramme. Was ist gesichert? Bundesgesundheitsbl Gesundheitsforsch Gesundheitsschutz 57:961-966.

3. von Wachter M, \& Hendrischke (2016) A Psychoedukation bei chronischen Schmerzen - Manual und Materialien. Springer-Verlag, Heidelberg

Samstag, 22.10.2017

\section{Individualisierte Schmerztherapie}

\section{SY26 - Schmerz, Schmerzempfindlichkeit und körpereigene Schmerzhemmung - Messung, Zusammenhänge und klinische Anwendung}

\section{R. Baron', R. Ruscheweyh' ${ }^{2}$,E. Pogatzki-Zahn ${ }^{3}$}

${ }^{1}$ Klinik für Neurologie, Universitätsklinikum Schleswig-Holstein, Campus Kiel, Sektion Neurologische Schmerzforschung und -therapie, Kiel, Deutschland, ${ }^{2}$ Klinikum der Universität München, Klinik und Poliklinik für Neurologie, München, Deutschland, ${ }^{3}$ Universitätsklinikum Münster, Klinik für Anästhesiologie, postoperative Intensivmedizin und Schmerztherapie, Münster, Deutschland

In den letzten Jahren wurden zahlreiche Methoden zur Messung von sensorischen Funktionen, Schmerzempfindlichkeit und Schmerzverarbeitung im zentralen Nervensystem entwickelt und an verschiedenen klinischen Anwendungen getestet.

Im Rahmen des Deutschen Forschungsverbundes Neuropathischer Schmerz (DFNS) wurde die standardisierte Quantitative Sensorische Testung (QST) entwickelt. Ziel war die Klassifizierung neuropathischer Schmerzpatienten anhand ihres sensorischen Profils und darauf basierend die Entwicklung einer mechanismenbasierten Therapie. In der Tat wurde kürzlich gezeigt, dass Oxcarbazepin bei Postzosterneuralgie besser bei Patienten mit „irritablem Nozizptor“-Profil wirkt als bei denen mit „Deafferenzierungs“-Profil. Auch Fragebögen wie z. B. der PainDetect erstellen sensorische Profile neuropathischer Schmerzpatienten. Außerdem erlauben sie ein schnelles und effektives Screening für das Vorliegen neuropathischer Schmerzkomponenten in der Praxis, z. B. bei Rückenschmerzpatienten.

Für die Untersuchung der individuell sehr unterschiedlichen Schmerzempfindlichkeit stehen die experimentelle Schmerzmessung sowie der „Pain Sensitivity Questionnaire“ (PSQ) zur Verfügung. Die präoperativ gemessene Schmerzempfindlichkeit kann die Schmerzhaftigkeit medizinischer Eingriffe vorhersagen und könnte daher bei der Planung einer optimalen postoperativen Schmerztherapie helfen. Bei Patienten mit unspe- zifischen chronischen Schmerzen findet sich eine generalisierte Erhöhung der Schmerzempfindlichkeit, was als Zeichen einer zentralen Sensibilisierung angesehen wird. Für neuropathische Schmerzen ist das weniger gut erwiesen und wird aktuell innerhalb des DFNS mithilfe des PSQ untersucht. Dabei interessiert insbesondere, ob Patienten mit nicht schmerzhaften Neuropathien ebenfalls eine erhöhte Schmerzempfindlichkeit haben. Mithilfe des PSQ wird außerdem untersucht, ob Depressionen unabhängig von gleichzeitig bestehenden Schmerzerkrankungen mit einer erhöhten Schmerzempfindlichkeit assoziiert sind.

Auch die endogene Schmerzhemmung ist individuell sehr unterschiedlich, und chronische Schmerzen sind mit einer reduzierten Aktivierbarkeit der endogenen Schmerzhemmung assoziiert. In den letzten Jahren ist insbesondere die absteigende Schmerzhemmung in den Fokus gerückt, die durch die Ausschüttung von Noradrenalin und Serotonin im Rückenmark schmerzhemmend wirkt. Diese kann beim Menschen nur indirekt gemessen werden, z. B. durch Verwendung des Conditioned Pain Modulation (CPM)-Tests oder der sogenannten Offset-Analgesie. Mithilfe dieser Methoden konnte z. B. gezeigt werden, dass schmerzmodulierend wirksame Antidepressiva mit Noradrenalin-/Serotonin-wiederaufnehmender Wirkung in der Tat bei den Patienten am wirksamsten sind, die eine schlecht funktionierende absteigende Schmerzhemmung haben. Intensiv untersucht wird außerdem, ob eine reduzierte absteigende Schmerzhemmung eine Prädisposition für die Entwicklung eines chronischen Schmerzes darstellt.

\section{Experimentelle Modelle und Pathophysiologie}

\section{SY27 - Das vegetative Nervensystem und Schmerz: Mögliche Zusammenhänge und therapeutische Optionen.}

\section{H. Schaible', F. Birklein ${ }^{2}$, A. Straube ${ }^{3}$}

${ }^{1}$ Friedrich-Schiller-Universität Jena, Institut für Physiologie I/Lehrstuhl für Neurophysiologie, Jena, Deutschland, ${ }^{2}$ Universitätsmedizin der Johannes Gutenberg-Universität Mainz, Klinik für Neurologie, Mainz, Deutschland, ${ }^{3}$ Klinikum Großhadern, Neurologische Klinik und Poliklinik, München, Deutschland

Das vegetative Nervensystem ist in die Regulation vieler Vorgänge des Körpers eingebunden. Dieses reicht von der Nahrungsaufnahme, Blutdruck-Kontrolle und Schlafregulation bis zur Beeinflussung von Entzündungsreaktionen und immunologischer Reaktionen.

In Bezug auf Schmerzerkrankungen ist es um das vegetative Nervensystem in den letzten Jahren eher ruhiger geworden, nachdem die Bedeutung von Eingriffen am Sympathikus im Rahmen therapeutischer Konzepte zur Behandlung des CRPS abgenommen hat. Andererseits finden sich zunehmend Befunde, die einen Einfluss autonomer Nervenfasern auf Entzündung und immunologische Vorgänge zeigen. Somit können sympathische und parasympatische Fasern - direkt oder indirekt - auch für die Schmerzentstehung von Bedeutung sein. Interessanterweise wird die Stimulation des Parasympathikus als therapeutische Option bei verschiedenen Kopfschmerz-Syndromen beschrieben.

Welche Wirkungen und Wirkungsmechanismen sind nun genau für den Sympathikus und Parasympathikus gezeigt worden? Wie gut lassen sich sympathische und parasympathische Wirkungen definieren? (Diese Frage stellt sich besonders dann, wenn Nerven wie der Vagusnerv elektrisch gereizt werden, denn viele Nerven enthalten sowohl efferente autonome als auch afferente sensorische Fasern). Lassen sich zentrale Effekte von peripheren abgrenzen? Welche therapeutischen Optionen wurden bisher durch Studien belegt und worauf beruhen diese Wirkungen? Diesen Fragen soll im Symposium nachgegangen werden.

H.-G. Schaible (Jena): Rolle des Parasympathikus bei Schmerzerkrankungen?

F. Briklein (Mainz): Rolle sympathischer Aktivität bei somatischen Schmerzen?

A. Straube (München): Bedeutung von Sympathikus und Parasympathikus bei primären Kopfschmerzen 


\section{Muskuloskelettale Schmerzen}

\section{SY28 - Paradigmenwechsel beim Postnukleotomie-Syndrom}

\section{Rauschmann', H. Casser ${ }^{2}$, M. Hüppe}

'Orthopädische Universitätsklinik Friedrichsheim gGmbH, Wirbelsäulenorthopädie, Frankfurt, Deutschland, ${ }^{2}$ DRK SchmerzZentrum Mainz, Mainz, Deutschland, ${ }^{3}$ Universität zu Lübeck, Klinik für Anästhesiologie, Lübeck, Deutschland

1. Neue Aspekte zum Postnukleotomie-Syndrom aus Sicht des Operateurs, M. Rauschmann

2. Multimodales interdisziplinäres Therapieprogramm beim postoperativen Rückenschmerz, H.-R.Casser

3. Psychologische Risikofaktoren für postoperativen Schmerz und chronische Schmerzentwicklung, M. Hüppe

Als Postnukleotomie-Syndrom (Synonyme Postdiskotomie-Syndrom, Postdiskektomie-Syndrom, englisch Failed back Surgery Syndrome (FBSS)) bezeichnete man früher alle anhaltenden starken Beschwerden nach der Operation einer lumbalen Bandscheibe, die durch Segmentinstabilität oder Verwachsungen im Wirbelkanal auftreten (Krämer 1966). Aufgrund der multifaktoriellen Genese des Syndroms ist diese Definition heute für den chronischen postoperativen Schmerz nicht mehr relevant.

Im ersten Beitrag stellt Herr Rauschmann eine neue und zutreffendere Definition, gerade für die Schmerztherapie vor, den postoperativen chronischen Rückenschmerz, der folgendermaßen definiert ist:

- Postoperativer anhaltender Rücken und/oder Beinschmerz für mehr als 6 Monate

- Kein klares chirurgisches Korrelat, das den klinischen Symptomen entspricht

- Keine interdisziplinäre Übereinkunft bzgl. einer chirurgischen Indikation

Falls keine morphologisch klar identifizierbaren Probleme im Indexsegement (monosegmentale Pathologie!) mit eindeutigem klinischen Korrelat vorliegen, ist zunächst ein interdisziplinäres Assessment indiziert (Casser et al. 2014) unter Einbeziehung der chirurgischen Kollegen. Gründe für einen interdisziplinären Therapieansatz ist die Prozesseffizienz (Hart, 2013), die ökonomische Effizienz (Meints et al. 2013), die klinische Effektivität (Carrea et al. 2006) und der Nachweis der verbesserten Betreuung durch einen interdisziplinären Ansatz (Tronnier 2015). Die Ausgestaltung eines entsprechenden multimodalen Behandlungskonzeptes wird Thema des zweiten Beitrages von Herrn Casser sein.

Frau Klinger (Beitrag 3) geht der Frage nach, ob sich ein Postnukleotomie-Syndrom verhindern lässt. Psychologische Risikofaktoren für postoperative Schmerzen nach Wirbelsäulen-Operationen zeigen nach gegenwärtigem Forschungsstand drei Gruppen von Risikofaktoren: Negative psychische Faktoren, eine vorbestehende Schmerzchronifizierung und psychische Störungen. Im Falle elektiver Bandscheiben-Operationen bzw. auch anderer Wirbelsäulen-Operationen sollten diese Faktoren deshalb präoperativ erfasst und bei der OP-Indikation berücksichtigt werden. Ggf. ist zunächst ein multimodales Behandlungsprogramm vorzuziehen oder es kann vor dem chirurgischen Eingriff eine Schmerzpsychotherapie erwogen werden.

Im engen Dialog zwischen Operateur und Schmerztherapeuten sollte der Begriff „Postnukleotomie-Syndrom“ oder ähnliche Begriffe zugunsten einer konkreten Beschreibung der Problematik verlassen werden und der multifaktoriellen Genese auch therapeutisch Rechnung getragen werden.

\section{Translationale Schmerzforschung}

\section{SY29 - Welche Rolle spielen Entzündungsvorgänge bei unseren Schmerzpatienten?}

J. Gierthmühlen', M. Sisignano², V. Tronnier ${ }^{3}$

'Universitätsklinikum Schleswig-Holstein, Campus Kiel Klinik für Neurologie, Kiel, Deutschland, ${ }^{2}$ Institut für Klinische Pharmakologie, Klinikum der Goethe-Universität Frankfurt am Main, pharmazentrum frankfurt/ZAFES, Frankfurt am Main, Deutschland, ${ }^{3}$ Neurochirurgische Universitätsklinik, Lübeck, Deutschland

In diesem Symposium soll die Bedeutung der Entzündung in der Pathogenese der Schmerzentstehung, -unterhaltung und -chronifizierung betrachtet sowie die Möglichkeit dieses Ansatzes bei der Therapie akuter und chronischer Schmerzen diskutiert werden.

Im ersten Teil des Symposiums wird Frau PD Dr. Gierthmühlen („Die Rolle von Entzündungsprozessen bei der Schmerzpathogenese - Ein Überblick“) einen Überblick über die Rolle der Entzündung in der Pathogenese von Schmerzen aus tier-, humanexperimenteller und klinischer Sicht anhand aktueller Literatur geben. Hierbei soll insbesondere die Funktion von Zytokinen bei der Entstehung verschiedener klinisch sich äußernder somatosensorischer Symptome und sich daraus ergebender Therapiemöglichkeiten im Sinne einer mechanismen-basierten Therapie dargestellt werden.

Im zweiten Vortrag von Herrn Dr. Sisignano („Cortison und Co- Entzündungshemmer in der Schmerztherapie“) soll die orale Pharmakotherapie der Schmerzen in Bezug auf einen antientzündlichen Therapieansatz näher dargestellt werden. Cortison und nichtsteroidale Entzündungshemmer sind die weltweit meistverwendeten Schmerzmedikamente, doch ihre Wirkung ist limitiert und aufgrund ihrer Wirkmechanismen ist ihre Langzeitnutzung mit teils erheblichen Nebenwirkungen verbunden. Es sollen in diesem Vortrag unter anderem die folgenden Fragen diskutiert werden: Welchen Effekt haben diese Substanzen bei verschiedenen chronischen Schmerzformen? Sind sie für die Therapie einer neurogenen Entzündung geeignet? Und welche neuen Entzündungsmechanismen sind aus der jüngeren Forschung bekannt, die möglicherweise im Rahmen einer neuen Schmerztherapie nutzbar gemacht und künftig als Alternative zu Cortison und Co-Entzündungshemmer eingesetzt werden können?

Der dritte Vortrag von Herrn Prof. Tronnier („Neuromodulation chronischer Schmerzen. Spielen Entzündungsmediatoren eine Rolle?") fasst die pathophysiologischen Grundlagen in Bezug auf Entzündungsmechanismen und ihre potentielle Rolle für die Neurostimulationstherapie zusammen. Neurostimulationstechniken wie die epidurale Rückenmarkstimulation sind typischerweise nicht erfolgreich bei inflammatorischen Schmerzen. Auf der anderen Seite werden Schmerzen, die durch neurogene Entzündungsmechanismen entstehen, wie das Failed Back Surgery Syndrom oder das Komplexe Regionale Schmerzsyndrom therapeutisch gut beeinflusst. Neuere Stimulationstechniken, wie die Vagusnervstimulation werden nicht nur zur Schmerztherapie, sondern auch zur Therapie entzündlicher Erkrankungen wie Asthma experimentell eingesetzt. Insgesamt sollen Möglichkeiten der antientzündlichen interventionellen Therapie diskutiert werden.

Aufgrund des breit angelegten Themenbereiches richtet sich das Symposium damit sowohl an Kliniker und Pharmakologen, die Anregungen für therapeutische Optionen bekommen als auch an Wissenschaftler, die sich näher mit der Rolle der Entzündung innerhalb der Schmerzpathophysiologie beschäftigen möchten. 


\section{Kopfschmerz}

\section{SY30 - Umdenken erwünscht - zur Rolle der Psychologie in Pathophysiologie und Therapie der Migräne}

\section{P. Kropp ' , R. Klinger' ${ }^{2}$, T.Dresler ${ }^{3}$, B. Meyer' ${ }^{1}$, S. Christiansen} 'Institut für Medizinische Psychologie und Medizinische Soziologie, Rostock, Deutschland, ${ }^{2}$ Universitätsklinikum Hamburg-Eppendorf (UKE), Klinik und Poliklinik für Anästhesiologie, Bereich Schmerzmedizin und Schmerzpsychologie, Hamburg, Deutschland, ${ }^{3}$ Klinik für Psychiatrie und Psychotherapie, Psychophysiologie und Optische Bildgebung, Tübingen, Deutschland, ${ }^{4}$ Universität Hamburg, Fakultät EPB, Psychotherapeutische Hochschulambulanz Verhaltenstherapie, Hamburg, Deutschland

Bereits seit längerer Zeit ist bekannt, dass neben physiologischen auch psychologische Faktoren bei der Genese und in der Behandlung der Migräne beteiligt sind. Dabei ist erstaunlich, in welchem Ausmaß psychologische Faktoren jeweils wirksam werden. So bieten sich neben speziellen diagnostischen Möglichkeiten aus der Psychophysiologie interessante Behandlungsoptionen an, die mittlerweile auch in den Leitlinien Eingang gefunden haben.

Migränepatienten weisen eine verminderte kortikale Habituation auf, was oft in Zusammenhang mit vermehrter Stressbelastung interpretiert wird und als Migräneauslöser angesehen werden kann. Kann im Umkehrschluss ein Habituationstraining die Häufigkeit von Migräneanfällen reduzieren? Entspannung und Meditation können das Auslösen von Migräneanfällen verhindern. Hingegen können Erwartungseffekte selbst, wie die Angst vor dem nächsten Migräneanfall, diesen auslösen. Wie lassen sich diese Erwartungseffekte erkennen und so modifizieren, dass sie zur Reduktion der Migränehäufigkeit führen? Für die Behandlung ist alleine die Vermittlung dieser Informationen an die betroffenen Patienten, die unter Migräne leiden, ein erster relevanter therapeutischer Schritt. Er ist Voraussetzung zum Umdenken von einem rein somatischen zu einem bio-psycho-sozialen Krankheitsmodell. Die auf diesem Modell basierende Behandlung stellt die Selbstwirksamkeit der Patienten in den Vordergrund. Wie können Patienten mit Migräne lernen, diese selbst etwas gegen die Kopfschmerzen zu tun? Welchen Beitrag leistet ein kopfschmerzspezifisches Gruppenbehandlungsprogramm zur Kopfschmerzbewältigung im ambulanten Setting? Es wird gezeigt, dass eine spezifische psychologische Behandlung in der Lage ist, medikamentöse Behandlungseffekte zu ergänzen und zu optimieren. Es werden die langfristig signifikanten Veränderungen in der Kopfschmerzsymptomatik anhand der Follow-up Daten bis zu einem Jahr dargestellt ( $p<.001$ für durchschnittliche Kopfschmerzen, -frequenz, -beeinträchtigungen, Depressionen, Coping und Katastrophisierung). Umdenken erwünscht - diese vorgestellten Konzepte bieten eine Vielzah an psychologischen Behandlungsverfahren. Im Symposium werden diese Konzepte beim Auslösen und bei der Behandlung der Migräne anhand aktueller Studien vorgestellt und bewertet.

\section{Varia}

\section{SY31 - Multikulturelle Aspekte der Schmerztherapie: (Um)Denken erwünscht!}

S. Azad ' , U. Stamer' ${ }^{2}$, A. Kopp ${ }^{3}$

'Klinikum der Universität München, Schmerzambulanz und Tagesklinik, Anaesthesiologie, München, Deutschland, ${ }^{2}$ Inselspital Universität Bern, Klinik für Anästhesiologie und Schmerztherapie, Bern, Schweiz, ${ }^{3}$ Klinik für Anaesthesiologie und operative Intensivmedizin, Berlin, Deutschland

1. Schmerztherapie im Ausland- wo steht sie? Shahnaz Christina Azad, München

2. Süditaliener, Syrer, Indianer und Deutsche - One size fits all? Ulrike Stamer, Bern

3 Schmerztherapie ausländischer Patienten: eine Frage der Kultur- oder Soziosensibilität? Andreas Kopf, Berlin
Die schmerztherapeutische Versorgung ausländischer Patienten, insbesondere aus fernen Kulturkreisen gewinnt in Deutschland zunehmend an Bedeutung. Nicht nur aufgrund von Sprachbarrieren stellt sie häufig eine große Herausforderung dar. Die Kommunikation mit Patienten „fremder Kulturen“ über Schmerzen kann an eigenen und patientenseitigen Stereotypen oder Befangenheiten scheitern.

In Stellungnahmen zur Behandlung von Patienten mit Migrationshintergrund wird daher immer wieder eine „Kultursensibilität“ gefordert, um die Bedürfnisse der Patienten richtig einschätzen zu können - eine Forderung, die aufgrund der heterogenen Migrantenpopulation unbewältigbar erscheint. Für die Art der Kommunikation von Schmerzen und die Bereitschaft, Hilfe zu suchen und anzunehmen, spielen Erziehung, Kultur und soziales Umfeld eine entscheidende Rolle (Schmerzäußerung eines tapferen Indianers sozial nicht erwünscht).

Aber auch andere Faktoren wie die Bewertung und Versorgung von Beschwerden durch das Gesundheitssystem im Heimatland spielen eine wichtige Rolle, insbesondere hinsichtlich der Erwartungen der Patienten. Nicht zuletzt beeinflusst auch die ethnische Zugehörigkeit eines einzelnen Patienten Effektivität und Nebenwirkungen einer Pharmakotherapie. So können genetische Varianten, die z. B. spezifisch für Bevölkerungsgruppen aus bestimmten geographischen Regionen der Erde sind, Pharmakokinetik und Pharmakodynamik von Medikamenten/Analgetika verändern. Dies kann einerseits zur mangelnden Wirkung (Fehlinterpretation als mangelnde Compliance), aber auch zu vermehrten Nebenwirkungen von Medikamenten führen.

Frau Prof. Azad wird ihren Schwerpunkt vor allem auf den Stellenwert der Schmerztherapie im Ausland im Vergleich zu Deutschland legen, der letztlich auch die Erwartungen ausländischer Patienten maßgeblich beeinflusst. Frau Prof. Stamer wird auf die unterschiedliche Wirksamkeit von Medikamenten abhängig von der Ethnizität eines Patienten eingehen.

Herr Dr. Kopf stellt in seinem Vortrag - auch aufgrund der aktuellen Herausforderungen - Überlegungen vor, wie eine „kultursensible“ Kommunikation über Schmerzen und Schmerzbehandlungen gelingen könnte.

\section{Neue medikamentöse Verfahren in der Migränetherapie}

\section{SY32 - Das Neuropeptid CGRP als Biomarker, Schlüsselmediator und therapeutisches Target inner- und außerhalb der Migräne}

\section{Neeb' ${ }^{1}$ U. Reuter ${ }^{2}$, K. Meßlinger ${ }^{3}$}

${ }^{1}$ Charité Universitätsmedizin Berlin, Neurologische Klinik und Hochschulambulanz, Berlin, Deutschland, ${ }^{2}$ Charité Universitätsmedizin Berlin, Neurologische Klinik und Poliklinik, Berlin, Deutschland, ${ }^{3}$ Universität Erlangen-Nürnberg, Institut für Physiologie \& Pathophysiologie, Erlangen, Deutschland

1. CGRP als therapeutisches Target und Biomarker in der Migräne- $\mathrm{Ge}$ schichte und neueste Entwicklungen, Uwe Reuter, Berlin

2. CGRP und seine Funktion außerhalb der Migräne, Lars Neeb, Berlin

3. CGRP und seine Rezeptoren im trigeminovaskulären System. Wieso ist die Blockade des CGRP-Systems antinozizeptiv?, Karl Messlinger, Erlangen

Das Neuropeptid Calcitonin Gene-related Peptide (CGRP) gilt seit den frühen Arbeiten Ende der 80iger und Beginn der 90iger Jahre als der Schlüsselmediator in der Migräne. Die Entwicklung von den tierexperimentellen, zellkulturellen und klinischen Beobachtungen hin zur Definition von neuen, direkt in den Migräne-Mechanismus eingreifenden Substanzklassen wie den CGRP-Antagonisten und CGRP-Antikörpern ist das Ergebnis einer erfolgreichen Translation von Forschung zur Klinik. Insbesondere die Ergebnisse der neueren klinischen Studien zu den CGRP-Antikörpern lassen nicht nur auf eine baldige Erweiterung der Therapiemöglichkeiten der Migräne hoffen, sondern werfen auch neue Fragen zur Pathophysiologie der Migräne, dem Wirkort dieser Substanzklassen und potentiellen Interaktionen außerhalb des trigeminovaskulären Systems auf. 
Im ersten Teil dieses Symposium wird Herr PD Dr. Reuter einen Überblick über die geschichtliche Entwicklung von der Entdeckung von CGRP in seiner Funktion als Vasodilatator bis hin zum therapeutischen Target in der Migräne geben, die neuesten klinischen Ergebnisse zur Effizienz der CGRP- und CGRP-Rezeptor-Antikörper in der Migränetherapie zusammenfassen und einen Blick auf mögliche zukünftige Entwicklungen werfen.

Die bisherigen klinischen Daten zu den CGRP-Antikörpern zeigen eine geringe Nebenwirkungsrate auf. Dennoch ist CGRP ein Neuropeptid, das nicht nur innerhalb des trigeminovaskulären Systems und in der Migräne eine wichtige Rolle spielt, sondern auch außerhalb hiervon in anderen Organsystemen als Mediator und Signaltransmitter agiert. Im zweiten Teil des Symposiums wird von Herrn Dr. Lars Neebanhand der aktuellen Studienlage aufgezeigt werden, welche weiteren Funktionen CGRP im menschlichen Körper übernimmt, bei welchen anderen Pathologien CGRP beteiligt ist und welche potentiellen Interaktionen in diesen Systemen durch eine CGRP-Blockade denkbar sein könnten.

Auf der Grundlage von experimentellen Daten wird Herr Prof. Karl Messlinger im letzten Teil des Symposiums die möglichen Wirkorte von CGRP im trigeminovaskulären System erläutern. Da für CGRP unmittelbare nozizeptive Wirkungen nur im ZNS bekannt sind, CGRP-Antagonisten und Antikörper andererseits kaum die Blut-Hirnschranke überwinden, werden alternative Erklärungsmöglichkeiten für die antinozizeptive Wirkung einer Blockade des CGRP Systems in der Migräne erörtert.

\section{Varia}

\section{SY33 - „Schmerz oder kein Schmerz - das ist hier die Frage." Möglichkeiten und Grenzen der Erfassung von akuten und chronischen Schmerzen}

\section{U. Bingel', J. Kappesser ${ }^{2}$, U. Kaiser ${ }^{3}$}

'Uniklinik Essen, Neurologie, Essen, Deutschland, ${ }^{2}$ Institut für Klinische Psychologie und Psychotherapie, Gießen, Deutschland, ${ }^{3}$ Universitätsklinikum Dresden, UniversitätsSchmerzCentrum, Dresden, Deutschland

Schmerz als multidimensionales Geschehen ist zugleich eine individuelle Empfindung, die durch kognitive, behaviorale, motivationale und affektive Aspekte des Patienten ebenso beeinflusst wird wie durch den kulturellen, interpersonellen und situativen Kontext. Das stellt die Patienten selbst als auch ihre Beobachter bei der Beurteilung von Schmerzen vor eine große Herausforderung. Die Schmerzerfahrung qualitativ in Worte zu fassen oder auf einer Skala zu quantifizieren, wird von Patienten häufig als schwierig erlebt. Gleichzeitig sind der verbale und non-verbale Ausdruck einer Schmerzerfahrung für Beobachter wichtige Hinweise für die Schmerzbeurteilung. Eine Identifizierung von Parametern zur Erfassung von Schmerz könnte sowohl in Forschung und Therapieevaluation nutzbar sein. Im Rahmen des Symposiums sollen aktuelle Befunde zur Schmerzselbstbeurteilung sowie zur Schmerzfremdbeurteilung akuter und chronischer Schmerzen vorgestellt sowie die Komplexität und Problemfelder der jeweiligen Beurteilungsformen dargestellt werden. Implikationen für die klinische und wissenschaftliche Arbeit werden diskutiert.

Bildgebende Verfahren haben gezeigt, dass zentrale Aktivitäten bei einer Schmerzerfahrung sichtbar sind. Ulrike Bingel beleuchtet in ihrem Vortrag, inwieweit es mit modernsten bildgebenden und elektrophysiologischen Methoden möglich ist, das individuelle Schmerzerleben zu objektivieren und möglicherweise zu quantifizieren. Limitationen und Fallstricke werden diskutiert. Es werden verschiedene multivariate - sog. „brainreading"- Verfahren vorgestellt, die zu Ziel haben, aus der Hirnaktivität die Existenz und Intensität einer akuten Schmerzerfahrung abzuleiten. Der Beitrag von Judith Kappesser wird experimentelle Befunde zum Einfluss von Selbstbericht, Mimik und organischen Ursachen vorstellen und auf theoretische Hintergründe eingehen. Ergebnisse zeigten, dass verschiedene Beurteilergruppen (z.B. Pflegekräfte mit unterschiedlicher Berufs- erfahrung) durch unterschiedliche Gewichtung dieser Indikatoren zu jeweils anderen Ergebnissen gelangen. Die Bedeutung dieser Befunde für Schmerzbeurteilungen in der Klinik und die Forschung soll kritisch erörtert werden.

Ergänzend stellt Ulrike Kaiser in ihrem Beitrag Ergebnisse einer umfassenden, multizentrischen Validierung üblicher Messinstrumente für Schmerzintensität an einer Population chronischer Schmerzpatienten in multimodalen Einrichtungen dar. Untersucht wurden Änderungssensitivität, Reliabilität sowie verschiedene Aspekte der Validität. Befragungen an Patienten ergaben, dass die Interpretierbarkeit dieser Skalen bei chronischen Schmerzen eingeschränkt ist. Die Ergebnisse werden im aktuellen Kontext der internationalen Diskussion beleuchtet und eingeordnet. Es werden Implikationen zum Einsatz in Effektivitätsstudien abgeleitet.

\section{Neuropathischer Schmerz}

\section{SY34 - Mechanismen der Schmerzhemmung: neue Aspekte \\ C. Maihöfner', F. Birklein ${ }^{2}$, M. Schmelz ${ }^{3}$, T. Schlereth ${ }^{2}$}

'Klinikum Fürth, Chefarzt der Neurologischen Klinik, Fürth, Deutschland, ${ }^{2}$ Universitätsmedizin der Johannes Gutenberg-Universität Mainz, Klinik für Neurologie, Mainz, Deutschland, ${ }^{3}$ Inst. Anästhesiologie Mannheim, Univ. Heidelberg, Experimentelle Schmerzforschung, Mannheim, Deutschland

Titel der drei Beiträge mit Referenten (in alphabetischer Reihenfolge): F. Birklein (Mainz): Fehlgeleitete Schmerzhemmung bei Patienten mit chronischen Schmerzen

C. Maihöfner (Fürth): Neurophysiologische Aspekte der Schmerzhemmung: was ist wichtig für die Therapie?

M. Schmelz (Mannheim): Schneller oder langsamer: Schmerzhemmung und Schmerzverstärkung durch Modulation der Entladungsfrequenz von Nozizeptoren

Inhaltsbeschreibung:

Endogene Mechanismen der Schmerzhemmung bilden die Grundlage für eine erstaunliche Modulation der Verarbeitung nozizeptiver Reize. In den letzten Jahren haben sich hier eine Reihe von neuen Erkenntnissen in der Grundlagenforschung ergeben, die bereits erste Ansätze für neue Therapieverfahren absehen lassen.

Im ersten Vortrag (C. Maihöfner) wird gezeigt, welche neurophysiologischen Mechanismen der Schmerzhemmung beim Menschen eine Rolle spielen. Dies hat bereits heutzutage Implikationen für die Entwicklung von Therapiestrategien. Dazu zählen u.a. pharmakologische Verfahren, stimulative Methoden sowie auch einzelne Elemente der multimodalen Schmerztherapie. Die funktionelle Bildgebung ermöglicht dabei erstmals das „Sichtbarmachen“"von therapeutischen Effekten im menschlichen Gehirn.

Der zweite Beitrag (M. Schmelz) fokussiert auf die Modulation der Entladungsfrequenz von verschiedenen Klassen von Nozizeptoren und die dadurch veränderte Schmerzverarbeitung. Während bestimmte Nozizeptorenklassen auch bei sehr niedriger Entladungsfrequenz $(<1 \mathrm{~Hz})$ eine zentrale Sensibilisierung auslösen können, fehlt diese nach Entladung polymodaler Nozizeptoren auch bei hohen Frequenzen, zumindest bei gesunden Probanden. Die sich daraus ergebenden Möglichkeiten und Grenzen von Humanmodellen zur Schmerzhemmung für die Pathophysiologie von chronischem Schmerz werden aufgezeigt.

Im dritten Vortrag (F. Birklein) wird schließlich darauf eingegangen werden, inwiefern die endogene Schmerzkontrolle bei chronischen Schmerzerkrankungen gestört ist. Es werden dazu aktuelle Befunde bei schmerzhaften Polyneuropathien, CRPS und Fibromyalgie diskutiert. Außerdem wird versucht, Argumente zu finden, dass die insuffiziente Schmerzhemmung nicht nur eine Folge chronischer Schmerzen sondern auch ursächlich für die Schmerzen sein kann.

Der Zuhörer kann in diesem Symposium damit die grundlegenden Mechanismen der Schmerzhemmung kennen lernen. Es werden darüber hinaus erste therapeutische Möglichkeiten aufgezeigt, die auf diesen neuen Erkenntnissen basieren. 


\section{Tumorschmerz}

\section{SY35 - Umdenken nötig: der schwierige Tumorschmerzpatient als Herausforderung in der Schmerzmedizin}

\section{Schenk' S. Wirz², K. Kieseritzky³, N. Kohnen ${ }^{4}$}

${ }^{1}$ Gemeinschaftskrankenhaus Havelhöhe gGmbH, Anästhesie, Schmerztherapie, Palliativmedizin, Berlin, Deutschland, ${ }^{2}$ CURA - kath. Krankenhaus im Siebengebirge, Bad Honnef, Anästhesie, Intensivmedizin, Schmerzmedizin/Palliativmedizin, Bad Honnef, Deutschland, ${ }^{3}$ St. JosephStift Bremen, Psychologischer Dienst, Bremen, Deutschland, ${ }^{4}$ Inst. f. Geschichte der Medizin, Uni Düsseldorf, Köln, Deutschland

Das Missverständnis - Tumorschmerz bei „Flüchtlingen“. Wie geht der Schmerzmediziner mit kulturell bedingten Missverständnissen um? PD Dr. Kohnen, Köln

Die Verweigerung - wenn die Tumorschmerztherapie nicht zum eigenen Weltbild passt - Strategien der Patientenführung

Dr. Schenk, Berlin

Der Verdränger - was steht dahinter, wenn der Patient alle medizinischen Maßnahmen ablehnt?

Dipl. psych. Kieseritzky, Hamburg

Auch bei kompliziertem Tumorschmerz, z. B. mit inflammatorischer oder neuropathischer Komponente, kann man bei guter Kenntnis mit einer pathophysiologisch basierten Pharmakotherapie zumeist ein zufriedenstellendes Behandlungsergebnis erreichen. Dieses Symposium beschäftigt sich mit drei Patientengruppen, bei denen ein zufriedenstellendes therapeutisches Ergebnis aufgrund von Kommunikationsproblemen oder Missverständnissen nicht oder nur schwer zu erreichen ist. Ziel ist es, danach einen professionelleren und dann auch effektiveren Umgang mit den beschriebenen Patientengruppen zu erreichen.

Missverständnisse entstehen oft beim Umgang mit Menschen aus anderen Kulturkreisen. Das Thema ist praktisch sehr relevant hochaktuell, da im Jahre 2015 über eine Million Menschen aus dem orientalischen und afrikanischen Kulturkreis zu uns gekommen sind. Der Vortrag soll Hilfestellung leisten, das Schmerzverständnis von Menschen aus diesen Kulturkreisen besser zu verstehen.

Verweigerung angebotener sinnvoller therapeutischer Leistungen, also auch schmerztherapeutischer Leistungen entsteht oft dann, wenn dieses Angebot nicht zum eigenen Weltbild passt (,ich will keine Chemie ..."). Grund ist eine erlebte Gefährdung der inneren Kohärenz und Identität sowohl beim Patienten, als auch beim Behandler. Diese Situation hat das Potenzial, zu einer Konfliktsituation zwischen Patienten und Arzt zu führen. Verdrängung einer zu Recht als elementar bedrohlich empfundenen Krebserkrankung kann auch zu Problemen bei der Tumortherapie und auch bei der Schmerzbehandlung führen. Dieser Vortrag soll Hilfe beim Umgang mit Patienten geben, die sich in dieser schwierigen Lebenssituation befinden.

Bei den Referenten handelt sich um bekannte Kollegen, die sich mit den jeweiligen Themen tiefgehend beschäftigt haben und dazu häufig auf Symposia verschiedener Fachrichtungen referieren. Privatdozent Dr. Kohnen ist ausgewiesener Experte auf dem Gebiet interkultureller Aspekte der Medizin und hat mehrere Publikationen zum Thema Schmerzerleben in unterschiedlichen Kulturkreisen veröffentlicht. Dr. Schenk ist Schmerz- und Palliativmediziner mit profunder praktischer und wissenschaftlicher Erfahrung. Als eines schmerzmedizinischen Zentrums hat er mit „Der Verweigerungshaltung" von Tumorschmerzpatienten täglich zu tun. Dipl. psych. Kieseritzkiy ist als Verfasserin und Koautorin verschiedener Buchpublikationen zum Thema Psychoonkologie bekannt und wird die Hintergründe verdrängenden Schmerzverhaltens erläutern.

\section{Psychologische Verfahren}

\section{SY36 - Emotion, Motivation und Schmerz - eine (un)heilvolle Allianz?}

\section{Hermann', A. Rusu' ${ }^{2}$ R. Majeed ${ }^{3}$, S. Becker ${ }^{4}$}

'Justus-Liebig-Universität Gießen, Abteilung Klinische Psychologie\& Psychotherapie, Gießen, Deutschland, ${ }^{2}$ Ruhr-Universität Bochum, Abteilung für Medizinische Psychologie \& Medizinische Soziologie, Bochum, Deutschland, ${ }^{3}$ Justus-Liebig Universität Gießen, Abteilung für Klinische Psychologie und Psychotherapie, Giessen, Deutschland, ${ }^{4}$ Universität Mannheim, Otto-Selz-Institut für Angewandte Psychologie, Mannheim, Deutschland

Schmerz hat intrinsisch eine emotionale und motivationale Komponente. Der enge Zusammenhang zwischen Schmerzerleben und emotionalem Befinden ist klinisch von großer Bedeutung. Neben Angst sind insbesondere depressive Verstimmungen zu nennen, die durch Schmerz induziert werden können, aber umgekehrt Schmerz verstärken. Klinisch zeigt sich dies beispielsweise in der empirisch gut belegten hohen Komorbidität von Schmerz und Depression. Die zugrundeliegenden Mechanismen sind allerdings weniger gut untersucht, trotz klarer Implikationen für die Behandlung. Im Symposium sollen zwei Mechanismen beleuchtet werden: Zum einen sind dies kognitive Verzerrungen und deren Rolle bei der Wechselwirkung von Schmerz und depressiver Stimmung. Zum anderen werden (mal-)adaptive Emotionsregulationsstrategien im Umgang einerseits mit Schmerz und andererseits mit negativer Stimmung als möglicher mediierender Mechanismus vorgestellt. Motivationale Aspekte tragen entscheidend zur Aufrechterhaltung von Schmerz und damit verbundenen Funktionsbeeinträchtigungen bei. Speziell die Aussicht auf Belohnung ist ein schmerzhemmender Faktor, Beeinträchtigungen bei Entscheidungsprozessen und Lernen bei chronischem Schmerz lassen sich vermutlich auf Defizite in der Verarbeitung von Belohnungsreizen zurückführen und sind deshalb unmittelbar klinisch relevant.

Im ersten Beitrag „Kognitive Verzerrungen und Depressivität bei chronischem Schmerz" (Rusu) liegt der Fokus auf kognitiven Mechanismen und speziell kognitiven Verzerrungen, die zur Aufrechterhaltung und Verstärkung einerseits von Depressivität und andererseits von schmerzbedingter Funktionsbeeinträchtigung beitragen. Basierend auf theoretischen Modellen werden empirische Befunde vorgestellt und Handlungsempfehlungen für die klinische Praxis abgeleitet.

Im zweiten Beitrag „(Mal)adaptive Emotionsregulationsstrategien im Umgang mit Schmerz und negativer Stimmung bei chronischem Schmerz" (Majeed \& Hermann) wird ausgehend von theoretischen Überlegungen die transdiagnostische Relevanz von Emotionsregulation im Kontext von Schmerz aufgezeigt. Empirische Befunde zum Vergleich zwischen verschiedenen adaptiven Emotionsregulationsstrategien im Umgang mit Schmerz werden im Überblick zusammengefasst und eigene neue Arbeiten bei Patienten mit chronischen Schmerzen speziell zur Rumination als einer besonders für die Aufrechterhaltung von depressiver Verstimmung, aber möglicherweise auch von Schmerz bedeutsamen Emotionsregulationsstrategie werden vorgestellt.

Im dritten Beitrag „Schmerz und Belohnung - eine komplexe Wechselwirkung" (Becker) steht die schmerzhemmende Wirkung der Aktivierung des Belohnungssystems im Vordergrund. Auf Grundlage von neurobiologischen, aber auch Verhaltensdaten werden die Mechanismen (z.B. Transmitter, neuronale Netzwerke) des inhibitorischen Einflusses von Belohnung auf das Schmerzerleben vorgestellt und die klinische Bedeutung anhand von Patientendaten aufgezeigt. 


\section{Varia}

\section{SY37 - Pathophysiologie, Diagnostik und Therapie chronischer Unterbauchschmerzen der Frau - eine interdisziplinäre Perspektive}

\section{S. Elsenbruch', U. Wesselmann ${ }^{2}$, C. Kolb ${ }^{3}$, F. Siedentopf $f^{4}$}

'Institut für Medizinische Psychologie, Verhaltensimmunbiologie, Essen, Deutschland, ${ }^{2}$ University of Alabama at Birmingham, Department of Anesthesiology and Perioperative Medicine, Birmingham, USA, 3Psychosomatischen Fachklinik, Bad Dürkheim, Bad Dürkheim, Deutschland, ${ }^{4}$ Martin-Luther-Krankenhaus, Klinik f. Gynäkologie und Geburtshilfe, Berlin, Deutschland

Hintergrund \& Ziele: Chronische Unterbauchschmerzen sind immer noch ein Stiefkind der Schmerzmedizin, obwohl sie für verschiedene medizinische Fachgebiete klinisch hoch relevant sind. Den deskriptiven Diagnosen "chronische Unterbauchschmerzen der Frau, Reizdarmsyndrom, chronisches Beckenbodenschmerzsyndrom, chronisches Harnblasenschmerzsyndrom" liegen klinisch heterogene Krankheitsbilder zu Grunde. Allen gemeinsam ist ein komplexes Zusammenwirken biologischer und psychosozialer Faktoren bei der Entstehung, Chronifizierung und Aufrechterhaltung der Symptomatik. Die Notwendigkeit einer interdisziplinären Diagnostik und multimodalen Therapie wird in Leitlinien und Übersichtsartikeln meist gefordert, in der klinischen Routineversorgung jedoch häufig nicht umgesetzt. In dem gemeinsamen Symposium der Arbeitskreise „Schmerz und Bewegung“ und „Viszerale Schmerzen“ werden neue Erkenntnisse zum chronischen Unterbauchschmerzen der Frau in Hinblick auf Pathophysiologie, Diagnostik und Therapie aus einer interdisziplinären Perspektive vorgestellt. Neuen Erkenntnissen aus der translationalen Schmerzforschung folgt die Vorstellung aktueller deutscher und internationaler Leitlinien in ihrer Bedeutung für Schmerzmediziner. Abschließend werden Möglichkeiten physiotherapeutischer Diagnostik und Therapie vorgestellt, die für viele Ärztinnen und Ärzte terra incognita ist.

\section{Vortragstitel \& Referenten:}

1. Urogenitale Schmerzsyndrome der Frau - von der Grundlagenforschung in die Schmerztherapeutische Praxis. Frau Prof. Wesselmann, Professor of Anesthesiology, Neurology and Psychology, Division of Pain Medicine, University of Alabama at Birmingham, U.S. A., wird aktuelle Daten der translationalen Schmerzforschung zu peripheren und zentralen Mechanismen der Chronifizierung urogenitaler Schmerzsyndrome vorstellen. Eine kritische Analyse dieser Forschungsergebnisse ist ein erster Schritt, um die klinischen Forschungsmodelle zu verbessern und individuelle Therapieentscheidungen bei der Behandlung zu optimieren.

2. Aktualisierte S2k - Leitlinie zum chronischen Unterbauchschmerz der Frau - was ist für Schmerzmediziner relevant? Frau Priv.-Doz. Dr. Siedentopf, Fachärztin für Gynäkologie und Geburtshilfe, Martin-LutherKrankenhaus Berlin, wird die aktualisierte S2-Leitlinie zum chronischen Unterbauchschmerz der Frau vorstellen und darlegen, welche diagnostischen und therapeutischen Maßnahmen für Nicht-Frauenärzte von Bedeutung sind.

3. Physiotherapeutische Diagnostik und Therapie bei chronischen Beckenschmerzen. Frau Kolb, leitende Physiotherapeutin in der Psychosomatischen Fachklinik Bad Dürkheim mit Schwerpunkt sozial-medizinische Funktionsdiagnostik und Behandlung von Schmerz- und Angst/ Panikpatienten, wird physiotherapeutische Untersuchungstechniken der Beckenbodenfunktion und -kraft sowie Behandlungstechniken wie viszerale Mobilisation, Triggerpunktbehandlung und Beckenbodentraining darstellen.

\section{Kopfschmerz}

\section{SY38 - Physiotherapie bei Migräne und Kopfschmerzen - Grundlagenforschung, Untersuchungsergebnisse und Behandlungsmöglichkeiten}

\section{Schüler ${ }^{1}$ K. Lüdtke ${ }^{2}$, B. Schäfer ${ }^{3}$}

${ }^{1}$ Klinik für Nephrologie und Hypertensiologie, Erlangen, Deutschland, ${ }^{2}$ Universitätsklinikum Hamburg Eppendorf, Institut für Systemische Neurowissenschaften, Hamburg, Deutschland, ${ }^{3}$ Migräne- und Kopfschmerzklinik Königstein, Physiotherapie, Mainz, Deutschland

Markus Schüler: Bei der Entstehung und Aufrechterhaltung primärer und sekundärer Kopfschmerzen spielt die trigeminale Innervation der Meningen eine entscheidende Rolle. Neben zentralen Mechanismen und nozizeptiven Vorgängen in den Hirnhäuten belegen klinische und experimentelle Beobachtungen aber auch einen erheblichen Einfluss peripherer extrakranialer Strukturen als möglichen Entstehungsort von Kopfschmerzen. Anatomische Studien an trigeminalen und zervikalen Afferenzen identifizierten die zugehörigen Ursprungsneurone und die peripheren Innervationsgebiete. Funktionelle Untersuchungen belegen die Existenz einer direkten peripheren Verbindung zwischen dem intra- und dem extrakranialen Kompartiment über kollaterale trigeminale Afferenzen sowie eine gesteigerte Empfindlichkeit zervikaler Strukturen im Einflussbereich des N. occipitalis nach meningealer Reizung. Die Ergebnisse verdeutlichen den Einfluss pathologischer Vorgänge in perikranialen Geweben auf die Pathogenese von Kopfschmerzen und zeigen, warum die Beeinflussung dieser Strukturen bei der Therapie von Kopfschmerzen hilfreich sein kann. Kerstin Lüdtke: Eine Vielzahl von Publikationen beschreibt einen Zusammenhang von Nackenschmerzen und primären Kopfschmerzarten, aber ist der Schmerz im Nacken nur ein Symptom oder gibt es tatsächlich Veränderungen im Bereich der Halswirbelsäule, die typisch für bestimmte Kopfschmerzarten sind? In einer Diagnostikstudie $(n=150)$ wurden Patienten mit Migräne und Spannungskopfschmerzen und gesunde Kontrollpatienten von einem verblindeten Physiotherapeuten auf muskuloskeletale Befunde untersucht. Dabei zeigten 9 von 11 Tests einen signifikanten Unterschied zwischen Kopfschmerzpatienten und Kontrollpersonen. Und was passiert, wenn diese Veränderungen physiotherapeutisch behandelt werden? Erste Pilotdaten $(n=12)$ zeigen, dass sich nur eine geringe Reduktion von Kopfschmerztagen pro Monat erzielen lässt, Patienten aber global eine deutliche Verbesserung der Kopfschmerzen angeben, die sich vor allem darin äußert, dass beginnende Kopfschmerzen seltener zu einer behandlungsbedürftigen Attacke werden.

Benjamin Schäfer: Die physiotherapeutische Behandlung von Patienten mit Migräne- und Kopfschmerzen im Rahmen der Multimodalen Schmerztherapie setzt ein umfassendes Assessment voraus. In der Therapie gilt es die Ursache der muskuloskeletalen Befunde aufzuspüren und wenn möglich, den Betroffenen so zu instruieren, dass er die Symptomatik eigenständig beeinflussen kann. Dies kann durch edukationsbasierte Übungstherapie (z. B. Antagonistentraining zum Ausgleich muskulärer Dysbalancen) erreicht werden. Unterstützend können Manuelle Therapie oder Triggerpunkttherapie angewandt werden. Neben den individuellen Aspekten sollte ein adäquates Ausdauertraining (z. B. Graded Activity oder Intervalltraining) und ein Entspannungsverfahren angeleitet werden. Mit diesem vorwiegend aktiven Konzept soll die Ursache einer hohen muskulären Anspannung als beitragender Faktor der Migräne/Kopfschmerzen nachhaltig verbessert werden. 


\section{Schmerz nach Operation}

\section{SY39 - Schmerzmanagement auf Intensivstationen nach Operationen - Möglichkeiten und Grenzen}

\author{
N. Nestler', I. Gnass', J. Freyhoff 2 \\ 'Paracelsus Medizinische Privatuniversität, Institut für Pflegewissenschaft \\ und -praxis, Salzburg, Österreich, ${ }^{2}$ Marienkrankenhaus Schwerte gem. \\ $\mathrm{GmbH}$, Abteilung für Anästhesiologie und Intensivmedizin, Schwerte, \\ Deutschland
}

Schmerzerfassung auf der Intensivstation- was passiert tatsächlich Irmela Gnass, Salzburg

Schmerztherapeutische Konzepte - zwischen Anspruch und Wirklichkeit Dr. Jörg Freyhoff, Schwerte

Schmerzmanagement auf der Intensivstation - nachhaltig sichern Nadja Nestler, Salzburg

Patienten werden nach großen Operationen oder Traumata auf Intensivstationen behandelt und oftmals steht das Überleben dieser Patienten im Zentrum der Bemühungen aller Professionen. Seit einigen Jahren ist nun aber auch das Schmerzmanagement deutlich in den Fokus gerückt, da Erkenntnisse um das Schmerzerleben dieser Patientengruppen vorhanden sind. Die besonderen Probleme dieser Patienten machen dabei ein hochdifferenziertes Vorgehen notwendig. Das Symposium wird die Möglichkeiten und Herausforderungen für ein berufsgruppenübergreifendes Schmerzmanagement aus unterschiedlichen Perspektiven darstellen.

Die Schmerzerfassung als ersten Schritt des Schmerzmanagements bedarf einer differenzierten Betrachtung. Frau Gnass wird in ihrem Vortrag die Möglichkeiten der Schmerzerfassung bei wachen, ansprechbaren Patienten wie auch Patienten, die aufgrund von bewusstseins- und/oder kognitiven Einschränkungen einer Fremdeinschätzung bedürfen, vorstellen. Die Ergebnisse von Beobachtungen auf Intensivstationen und Interviews mit pflegerischen und ärztlichen Mitarbeitern geben zudem Aufschluss über den gegenwärtigen Einsatz von Skalen, die Umsetzung von Aufwach- oder Spontanatemversuchen sowie zur Kommunikation und Dokumentation von Schmerz. Die hieraus abgeleiteten Interventionen und deren Konsequenzen für die Schmerztherapie bzw. deren Einfluss auf die schmerztherapeutische Ergebnisqualität werden diskutiert.

Die gültige aktualisierten Leitlinie „Analgesie, Sedierung und Delirmanagement in der Intensivmedizin" (2015) zeigt Möglichkeiten einer adaptierten medikamentösen Schmerztherapie. Diese Therapieoptionen und ihre Umsetzung in neuen patientenadaptierter Schmerzkonzepte werden von Herrn Dr. Freyhoff dargestellt. Damit werden Wege für eine suffiziente Schmerztherapie erörtert, die die Rate an postoperativen Komplikationen reduzieren.

Um die eingeführten Konzepte für ein berufsgruppenübergreifendes Schmerzmanagement auf der Intensivstation nachhaltig zu sichern, bedarf es auch hier eines systematischen Schmerzmanagements. Unabhängig, ob eine externe Qualitätsprüfung durch eine Zertifizierung angestrebt wird oder die Klinik „nur“ das Schmerzmanagement für ihre Patienten langfristig sichern will, bedarf es systematischer qualitätssichernder Strukturen und Prozesse, um dies sicher zu stellen. Frau Nestler wird Möglichkeiten solcher Rahmenbedingungen und Inhalte und daraus resultierende Ergebnisse präsentieren.

\section{Translationale Schmerzforschung}

\section{SY40 - Hyperalgesie und Allodynie: Von der Pathophysiologie zur klinischen Relevanz}

\section{Pereira' ', J. Gierthmühlen², E. Enax-Krumova ${ }^{3}$}

'Universitätsklinikum Münster, Klinik für Anästhesiologie, operative

Intensivmedizin und Schmerztherapie, Münster, Deutschland,

2Universitätsklinikum Schleswig-Holstein, Campus Kiel Klinik für Neurologie, Kiel, Deutschland, ${ }^{3}$ BG Universitätsklinikum Bergmannsheil, Neurologische Klinik, Bochum, Deutschland

Titel der Vorträge:

1. Hyperalgesie in humanen experimentellen Schmerzmodellen - alle(s) gleich?, M. Pereira, Münster

2. Dynamische und statische mechanische Hyperalgesie bei Patienten mit neuropathischen Schmerzen - alles Zufall?, J. Gierthmühlen, Kiel

3. Hyperalgesieerfassung im Konzept der mechanismen-basierten Therapie - alles klar?, Elena Enax-Krumova, Bochum

Sensorische Funktionssteigerungen wie Hyperalgesie und Allodynie spiegeln eine erhöhte Reaktivität des zentralen Nervensystems (e.g. Sensitisierungsprozesse im peripheren und zentralen Nervensystem) wider und sind damit eng verbunden mit verschiedensten klinischen relevanten Schmerzen völlig unterschiedlicher Genese. Allerdings sind Auftreten, Verteilungsmuster und Bedeutung von Hyperalgesie und Allodynie für Schmerzen unterschiedlicher Pathogenese sehr unterschiedlich und sogar innerhalb gleicher Krankheitsbilder oft sehr uneinheitlich. Ziel des Symposiums soll es, einen translationalen Überblick über Erkenntnisse zu Hyperalgesie und Allodynie aus den letzten Jahren zu geben ihre Bedeutung für pathophysiologische Zusammenhänge, Diagnostik und Therapie von (nicht nur neuropathischen) Schmerzen zu geben.

Vortrag 1.: Humane Schmerzmodelle können wichtige Hinweise zu pathophysiologischen Fragestellungen bezüglich Schmerz unterschiedlicher Genese geben. Im ersten Vortrag sollen neue Erkenntnisse zu pathophysiologischen Zusammenhänge der Phänomene Hyperalgesie und Allodynie dargestellt, das unterschiedliche Auftreten von Hyperalgesie und Allodynie sowie deren Modifizierbarkeit in verschiedenen experimentellen Schmerzmodellen verglichen und eine mögliche Bedeutung für bestimmte Schmerzprozesse herausgearbeitet werden.

Vortrag 2: Patienten mit neuropathischen Schmerzen weisen z. T. ein sehr unterschiedliches Muster verschiedener Funktionssteigerungen auf das z. T. auch innerhalb eines Krankheitsbildes stark variiert. Im zweiten Vortrag wird diskutiert, ob das Konzept der verschiedenen Arten der Funktionssteigerungen, e.g.g mechanischen Hyperalgesie/Allodynie (statisch oder dynamisch, spitze oder stumpfe mechanische Hyperalgesie/Allodynie) sich auf die Klinik bei Patienten mit neuropathischen Schmerzen übertragen lässt und ob spezifische Formen der mechanischen Hyperalgesie/Allodynie mit bestimmten Krankheitsbildern oder auch untereinander assoziiert sind.

Vortrag 3: Die Therapie neuropathischer Schmerzen ist gekennzeichnet durch eine hohe Number-needed-to-treat-Rate (im Durchschnitt 1,9-10,6). Die Unterschiede der Behandelbarkeit von Patienten werden durch verschiedene zugrundeliegenden Mechanismen erklärt. Erste Studien geben nun Hinweise für die Abhängigkeit der Therapieerfolgsrate von einer nachweisbaren sensorischen Funktionssteigerung (z. B. Hyperalgesie bestimmter Modalität). Frau Dr. Enax-Krumova wird in ihrem Vortrag zusammenfassen, welche Relevanz der Nachweis einer thermischen oder mechanischen Hyperalgesie für den klinischen Alltag hinsichtlich der Auswahl eines geeigneten Analgetikums bei Patienten mit neuropathischen Schmerzen hat. 


\section{Workshops}

\section{Donnerstag, 20.10.2016}

\section{WS01 - „Ich bilde mir den Schmerz doch nicht ein“ - Bio-psycho- soziale Zusammenhänge von Schmerz erklären - aber wie?}

\section{H. Nobis ${ }^{1}$}

'MEDIAN-Klinik am Burggraben, Orthopädische Psychosomatik/

Interdisziplinäre Schmerztherapie, Bad Salzuflen, Deutschland

Hintergrund der Thematik: Wer die Arbeit mit chronisch Schmerzkranken kennt, weiß um die besondere Herausforderung sowohl im Einzelkontakt als auch in der Gruppenarbeit, besonders dann, wenn bio-psycho-soziale Zusammenhänge angesprochen werden. „Vorurteile" auf Seiten des Patienten, Schmerz sei ein lokales Geschehen, Schmerz weise immer auf einen körperlichen Defekt und ihre Skepsis bis Ablehnung gegenüber psycho-sozialen Mitwirkungsfaktoren führen, wenn psycho-soziale Wirkfaktoren angesprochen werden, schnell zu Kommunikationsproblemen, wenn nicht gar zum Abbruch der Behandlung. Der dann oft vom Schmerzpatienten unter heftiger Empörung geäußerte Vorwurf lautet: „Ich bilde mir den Schmerz doch nicht ein“.

Mehrere Autoren [3, 6]. konnten in Studien nachweisen, dass jeder Mensch in der Lage ist, Zusammenhänge der Schmerzphysiologie zu verstehen und dass Kenntnisse bio-psycho-sozialer Zusammenhänge dazu führen, dass die Schmerzen als weniger bedrohlich wahrgenommen werden und sich der Umgang mit Schmerzen verbessert.

Zur Bestätigungen der Bedeutung von Information und Edukation in einer multimodalen Schmerztherapie sei auf eine Veröffentlichung der "American Geriatrics Society" (AGS) hingewiesen. So steht in den evidence geprüften Leitlinien („General Principles“):,Patient education programs are integral components of the management of persistent pain syndromes". (JAGS, 50, 2002). Basierend auf einem Review von 24 randomisierten, kontrollierten Studien [2] konnte gezeigt werden, dass bei Patienten mit (sub)akutem Rückenschmerz bereits eine 2,5-stündige Edukation ausreicht, um die Chancen auf eine Rückkehr an den Arbeitsplatz zu verbessern.

Die IASP unterstrich die grundlegende Bedeutung von Informationen für Schmerzkranke auf ihrem Welt-Schmerzkongress 2010 mit der „Declaration of Montreal“, wo sie im Artikel 2 das weltweite Recht auf Informationen über Diagnose und Behandlungsmöglichkeiten proklamierten (www.iasppain.org). Zeitgleich wurde in Deutschland die „Nationale Versorgungsleitlinie Kreuzschmerz" veröffentlicht (www.versorgungsleitline.de), die eine adäquate, individuelle Information und Beratung bei akutem/subakutem und Schulungsmaßnahmen bei chronischem Kreuzschmerz ausdrücklich empfiehlt und diese Maßnahmen als Bestandteile einer Regelversorgung ausdrücklich einfordert.

Es wurden eine Reihe von standardisierten Behandlungsmanuale veröffentlicht (u. a. Pfingsten, Basler, Kröner-Herwig, Egle, Flor), die bei näherer Betrachtung und je nach therapeutischer Fachrichtung unterschiedliche Aspekte einer Informationsvermittlung hervorheben und in Studien positive Behandlungseffekte erzielten.

In einem multimodalen Behandlungskonzept wird daher der Schmerzedukation eine Schlüsselrolle zugestanden.

Die dafür notwendigen Arbeitsweisen haben wir in unserer Primär-Ausbildung nicht vermittelt bekommen. Das Begreifbarmachen eines „biopsycho-sozialen“ Schmerzverständnisses kann auch an einer „pädagogisch" unzureichenden Vermittlung scheitern [7]. Deshalb ist es wichtig $[4], \ldots$, dass Erklärungen für die Patienten verständlich sind und möglichst viele ihrer alltäglichen Erfahrungen aufgreifen."

Lebensnähe und Plausibilität von Erklärungen (Nobis 2012) führen zu einer Hinterfragung des eigenen Schmerzkonzepts und schaffen damit erst die Motivation und Compliance für ein interdisziplinär-multimodal ausgerichtetes Therapiekonzept.

Lernziele: Den Schmerz und besonders den chronischen Schmerz als bio-psycho-soziales Phänomen für den Patienten „begreifbar“ machen,
Zusammenhänge „auf Höhe des Patienten“ erklären zu können heißt, Schmerzedukation auch als eine „pädagogische“ Herausforderung anzuerkennen und mit Prinzipien der Pädagogik zu gestalten.

Inhaltlicher Ablauf: Die Bedeutung der (Schmerz)-Edukation wird zunächst anhand von klinischen Erfahrungen und wissenschaftlichen Daten untermauert. Auszüge aus der sich in der Praxis bewährten Erklärungsmodellen zum Thema „Der bio-psycho-soziale Schmerz" und „Wie wird aus Schmerz - chronischer Schmerz?" des Bad Salzufler Schmerzwerkstatt werden vorgestellt und deren Umsetzung in ein Einzel- oder Gruppensetting diskutiert.

Teilnehmerkreis: Pflegekräfte, Ärzte, Psychologen und Physiotherapeuten

Literatur

1. American Geriatrics Society (2002) The Management of Persistent Pain in Older Persons. JAGS 50:205-224

2. Engers $A J$ et al (2011) Individual patient education for low back pain. The Cochrane Collaboration. The Cochrane Library 2011(2). John Wiley \& Sons, Hoboken, NJ, Cochrane Database Syst Rev. CD004057. doi: 10.1002/14651858.CD004057.pub3

3. Butler DS, Moseley GL (2005) Schmerzen verstehen. Springer, Heidelberg

4. Hildebrandt J, Pfingsten M et al (2003) Das Manual (GRIP). congress compact, Berlin

5. Kröner-Herwig B et al (2011) Spezielle Schmerzpsychotherapie. Springer, Heidelberg

6. Moseley GL (2004) Evidence for a direct relationship between cognitive and physical change during an education intervention in people with chronic low back pain. Euro J Pain 8:39-45

7. Nobis HG (2013) "Ich bilde mir den Schmerz doch nicht ein" - Woran Edukationskonzepte scheitern können. In: Arbeitskreis Klinische Psychologie in der Rehabilitation BDP (Hrsg.) (Selbst-)Konzepte bei veränderten Lebensbedingungen. Deutscher Psychologen Verlag, Berlin

8. Nobis HG, Pielsticker A (2013) Ärztliche Edukation und Kommunikation in der primären Schmerzbehandlung. Schmerz 27:317-324

9. Nobis HG, Pielsticker A (2016) Information und Edukation des Patienten. In: Casser HR, Hasenbring M, Becker A, Baron R (Hrsg.) Rückenschmerzen und Nackenschmerzen. Springer, Heidelberg

10. Nobis HG et al (2016) Schmerz - eine Herausforderung. Informationen für Betroffene und Angehörige. Springer, Heidelberg

\section{WS02 - Opioide in der Praxis}

M. Gleim, S. Schulzeck

Universitätsklinikum Schleswig-Holstein, Campus Kiel, Klinik für

Anästhesiologie u. operative Intensivmedizin, Kiel, Deutschland

Langzeit-Opioid behandelte Patienten begegnen uns zunehmend häufig auch außerhalb der speziellen schmerztherapeutischen Versorgung. Besonders im akut medizinischen Bereichen wie dem ambulanten Bereitschaftsdienst, der Notaufnahme oder der perioperativen Schmerztherapie stellen sich hierbei häufig Probleme.

Im Workshop werden grundlegende Probleme chronisch Opioid behandelter Schmerzpatienten bearbeitet. Spezielle Komplikationen der OpioidLangzeittherapie, deren pathophysiologische und psychosoziale Grundlagen und Lösungsmöglichkeiten werden anhand Erfahrungen aus dem eigenen Arbeitsbereich und exemplarischer Fälle dargestellt. Das „Wie“ und „Für" und „Wider" der möglichen Lösungsansätze in diesen Situationen wird mit den Teilnehmern diskutiert. Ein Schwerpunkt behandelt die Besonderheiten in der perioperativen Versorgung von Patienten unter einer chronischen Opioidtherapie und die hierbei nicht seltenen Probleme in der Zusammenarbeit mit Nicht-Schmerzspezialisten: Nicht be- oder erkannte Opioid-Vortherapie, die Probleme einer Absetzsymptomatik oder einer mangelnden Effektivität der sonst wirksamen Akutschmerztherapie erfordern spezielles Vorgehen und erhöhen den Aufwand der Versorgung dieser Patienten oft beträchtlich.

Die Teilnehmer des Workshops erfahren von den Besonderheiten und Folgen einer Opioid Langzeittherapie in der allgemeinen medizinischen Versorgung. Neben schmerztherapeutischen werden auch Kosten- und Vergütungsrelevante Aspekte dargestellt. 


\section{WS03 - Biofeedbacktherapie bei Kopfschmerzen und Migräne}

\section{P. Kropp, B. Meyer}

Institut für Medizinische Psychologie und Medizinische Soziologie, Rostock, Deutschland

Den aktuellen Leitlinien der Deutschen Migräne- und Kopfschmerzgesellschaft zufolge gelten psychologische Verfahren, die der Verhaltenstherapie entstammen, als evidenzbasiert und als sehr effektiv bei der Behandlung primärer Kopfschmerzen, wie Migräne und Kopfschmerz vom Spannungstyp. Psychologische Verfahren sind wirksam und können unter bestimmten Voraussetzungen als Alternative zu medikamentösen Therapie eingesetzt werden. Neben Entspannungsverfahren, operanten und kognitiv/verhaltenstherapeutischen Behandlungsansätzen hat sich seit vielen Jahren die Biofeedbacktherapie als verhaltenstherapeutische Maßnahme zur Behandlung von Kopfschmerzen und Migräne etabliert. Das Prinzip dieser Behandlung ist einfach: grundsätzlich können alle autonom oder zentral ablaufende Körperfunktionen über Biofeedback beeinflusst werden. Sie müssen nur bewusst wahrgenommen werden. Dadurch lassen sich diese Funktionen willentlich in die gewünschte Richtung beeinflussen. So kann bei der Migräne zumindest eine autonome Ruhigstellung erreicht werden, die ihrerseits die Wahrscheinlichkeit beispielsweise für einen Migräneanfall reduziert. Aber auch spezifischer wirkende Verfahren können bei der Behandlung von Kopfschmerzen eingesetzt werden, so beispielsweise ein Ansatz von Habituationseffekten.

Im Workshop werden in einem kurzen Vortrag die Grundsätze der Biofeedbacktherapie vorgestellt. Es folgen Fallbeispiele bei der Anwendung im Bereich chronischer Schmerzzustände, hier speziell bei der Migräne und beim Kopfschmerz vom Spannungstyp. Abgerundet wird das Seminar mit ausführlichen praktischen Übungen der Teilnehmer an verschiedenen, zur Verfügung gestellten Biofeedbackgeräten. Diese Gerätekunde bezieht auch neueste App's von Smartphones mit ein. Dabei werden Fallstricke der Geräte und bei der Behandlung ausführlich erläutert. Außerdem wird auf abrechnungstechnische Besonderheiten dieser Behandlungsmethode und auf mögliche Kontraindikationen eingegangen.

\section{WS04 - Der schwierige Fall - Ein Videoseminar zum praktischen Umgang mit Schmerzpatienten \\ C. Lahmann}

Klinikum Rechts der Isar, Klinik und Poliklinik für Psychosomatische Medizin und Psychotherapie, München, Deutschland

Chronische Schmerzpatienten gelten im klinischen Alltag oft als schwierige Patienten. Bei genauer Betrachtung stellt sich meist die Interaktion zwischen Ärzten und Patienten mit chronischen Schmerzen als wesentliches Problem dar. Die grundlegenden Schwierigkeiten gehen im Wesentlichen auf drei Aspekte zurück: Das Drängen der Patienten auf weitere somatische Diagnostik und Therapie mit konsekutiv deutlich appellativem Verhalten, die Befürchtung der Ärzte, vielleicht doch eine verborgende Krankheit zu übersehen oder übersehen zu haben sowie drittens die Diskrepanz in den jeweiligen Ursachenüberzeugungen. Patienten mit chronischen Schmerzbeschwerden sprechen gleichsam eine „Sprache der Schmerzen“; die Symptomklagen des Patienten sollten vom Therapeuten aktiv entgegengenommen und durch Nachfragen und Anregungen strukturiert werden. Die teils sehr ausführlichen Schilderungen der Schmerzen sollten dabei nicht als Widerstand, sondern unvermeidbares Charakteristikum dieser Patientengruppe verstanden werden. Aus dieser aktiv-stützenden Haltung entspringt insbesondere bei Patienten mit einer psychischen Komorbidiät die Form der tangentialen Gesprächsführung. Dies bedeutet, dass Bereiche des intrapsychischen Erlebens eher beiläufig angesprochen werden und die Patienten nicht vorschnell mit der Aussage konfrontiert werden, die Beschwerden seien teilweise oder gänzlich psychisch oder psychosomatisch zu erklären. Stattdessen kann ein positives Erklärungsmodell angeboten werden, z. B. mit Information über psychophysiologische Zusammenhänge wie veränderten Körperreaktionen bei Aufregung oder Stress.
Um eine konfrontative, psychische Aspekte zu sehr fokussierende Gesprächsführung zu vermeiden, bieten sich auch Verweise auf andere Patienten an: „Bei vielen meiner Kopfschmerz-Patienten ist es so, dass ...“. Einen ähnlichen Effekt haben sogenannte „Ich Botschaften“, z. B. die Formulierung: „Ich habe den Eindruck, dass die hartnäckigen und langanhaltenden Schmerzen Ihnen auch seelisch ziemlich zusetzen." Hier hat der Patient die Möglichkeit, diesem Eindruck zuzustimmen, aber auch ohne großes konfrontatives Moment zu verneinen.

Dies bedingt eine Anpassung der Gesprächsführung - insbesondere in der Frühphase der Arbeit mit dem Patienten - um die fast regelhaft zu erwartenden interaktionellen Schwierigkeiten zu minimieren. Hierzu ist eine zielgerichtete, rationale Kommunikationsstrategie hilfreich, die lehr- und lernbar ist und im Rahmen des Seminars mit Hilfe von Videosequenzen vermittelt wird.

\section{WS05 - Sonographie - Risikoreduktion für Patient und Therapeut durch sonographisch-gestützte Interventionen: gibt es wirbelsäulennah eine echte Rationale?}

\section{R. Likar', F. Reisig' ${ }^{2}$, T. Wilhelm ${ }^{3}$}

${ }^{1}$ Klinikum Klagenfurt, Zentrum für Interdisziplinäre Schmerztherapie und Palliativmedizin, Klagenfurt, Österreich, ${ }^{2}$ BG Unfallklinik Murnau, Anästhesie, Intensiv- und Schmerzmedizin, HBO Druckkammerzentrum, Murnau, Deutschland, ${ }^{3}$ Berufsgenossenschaftliche Unfallklinik Murnau, Abteilung für Schmerzmedizin, Murnau, Deutschland

Der Einsatz der Sonographie bei peripheren Blockaden ist im Bereich der perioperativen Akutschmerztherapie zum „common sense“ geworden. Es gibt nun eine Vielzahl von evidenzbasierten Daten, dass sich durch den Einsatz der Sonographie eine Verbesserung der Blockadequalität, eine verkürzte Anschlagszeit, weniger häufig akzidentielle Gefäßpunktionen, weniger Punktionsversuche und zufriedenere Patienten haben realisieren lassen [1]. Bezüglich der Inzidenz von Nervenschäden zeigt sich hier noch ein uneinheitliches Bild [2,3]. Eine Untersuchung an Auszubildenden zeigt, dass bei sonographisch-gestützten Blockaden zu Beginn Nadelkontrolle und Sondenführung oft ungenügend sind. Erst bei einer Durchführungshäufigkeit von mehr als 50 Blockaden ist die Koordination ausreichend [4]. In der Diagnostik und Therapie chronischer Schmerzen findet nun die Sonographie häufigeren Einsatz bei wirbelsäulennahe Blockaden, die lange Zeit die Domäne der Fluoroskopie und der Computertomographie waren [5]. Ob sich analog zu den peripheren Nervenblockaden ernsthafte Komplikationen wie Pneumothorax oder intravasale Injektionen vermeiden lassen, ist nicht systematisch erfasst. Sicher besteht ein erhebliches Einsparungspotential an Strahlenbelastung: zum einen für den Patienten, insbesondre bei Blockaden im Bereich von strahlensensiblen Organen wie Struma und kleines Becken, als auch für den durchführenden Arzt. Im Rahmen eines Kurzvortages sollen die klinischen Einsatzmöglichkeiten der Sonographie in der schmerzmedizinischen Diagnostik erarbeitet werden, mit einer kurzen Darstellung der aktuellen Studienlage und einer Einführung in die relevante Sonoanatomie. Im Anschluss daran kann an drei Arbeitsplätzen das theoretisch Erarbeitete unter Anleitung für je 30' in Form eines Live-Schalls am Probanden angewendet werden.

\section{Literatur}

1. Liu SS, Ngeow JE, Yadeau JT (2009) Ultrasound-guided regional anesthesia and analgesia: a qualitative systematic review. Reg Anesth Pain Med. 34(1):47-59

2. Seidel R, Natge U, Schulz J (2013) Distal sciatic nerve blocks: Randomized comparison of nerve stimulation and ultrasound guided intraepineural block. Anaesthesist 62:183-189

3. Vassiliou T, Eider J, Nimphius W, Wiesmann T, de Andres J, Müller HH, Wulf H, Steinfeldt T (2012) Dual guidance improves needle tip placement for peripheral nerve blocks in a porcine model. Acta Anaesthesiol Scand 56:1156-1162

4. Sites BD, Spence BC, Gallagher JD, Wiley CW, Bertrand ML, Blike GT. (2007) Characterizing novice behavior associated with learning ultrasound-guided peripheral regional anesthesia. Reg Anesth Pain Med. 32:107-115

5. Narouze S, Peng PW (2010) Ultrasound-guided interventional procedures in pain medicine: a review of anatomy, sonoanatomy, and procedures. Part II: axial structures. Reg Anesth Pain Med 35:386-396 


\section{WS06 - Akuter Schmerz-Hypnose; chronischer Schmerz- Hypnotherapie - Unterschiede und Ähnlichkeiten in der Behandlung-}

\section{Anne M. Lang}

AML Institut Systeme und Mllton Erickon Institut Bonn, Bonn, Deutschland

Sowohl beim akuten als auch beim chronischen Schmerz helfen hypnosuggestive Angebote. Wir haben es aber mit zwei verschiedenen Kontexten zu tun:

a) Beim akuten Schmerz geht es darum, eine aktuell begrenzte Situation gut zu über(be)stehen und sogar von dem Zustand in Trance zu profitieren. Hypnose hat hier die Aufgabe, den Patienten über Fokussierungen, Dissoziationen, Assoziationen, sprachliche und metaphorisch analoge Angebote in einen Modus zu führen, indem sich die Situation und das Schmerzempfinden psychisch-körperlich-mental-sozial anders gestaltet.

b) Bei einem chronischen Schmerz befinden wir uns im Setting einer Psychotherapie. Hier hilft Hypnotherapie dem Patienten für sich Möglichkeiten zu finden, sich wieder breiter fokussieren zu können, sich mental anders auszurichten und in eine persönliche Entwicklung zu gehen.

c) Interessant ist dann, wie sich die Anwendung von Hypnose, einmal als Situations-Hypnose- einmal als Hypno-Psychotherapie kontextspezifisch gestaltet.

So geht es in der MEG- Weiterbildung für „Medizinische Hypnose“ darum, wie Behandlungs- und Eingriffs-Situationen begleitet und erleichtert werden können. Der Kontext kann sehr gravierend sein, beinhaltet aber einen eher kurzen intensiv hypnotischen Kontakt.

Die MEG-Weiterbildung für „Klinische Hypnose/Hypnotherapie“ beinhaltet das Vorgehen, Hypnotherapie als Psychotherapie z. B. von Schmerzpatienten zu nutzen. Hier greifen die psychotherapeutischen Prinzipien des Einrichtens eines therapeutischen Systems und der Arbeit damit.

c) Das Erlernen der Selbsthypnose kann dann kontextübergreifend interessant sein.

Ein Beispiel aus dem ersten Setting und eines aus dem zweiten Setting werden das veranschaulichen.

Die Workshop Leiterin ist Lehrende für „Medizinische Hypnose“ und „Klinische Hypnose“ der Milton Erickson Gesellschaft MEG.

\section{WS07 - Die multimodale Diagnostik und Therapie des Chronischen Rückenschmerzes}

\section{B. Maurus ${ }^{1}$, J. Mallwitz², M. Richter ${ }^{3}$}

${ }^{1}$ Rückenzentrum Am Michel Hamburg, Tagesklinik für akute und chronische Schmerzpatienten, Hamburg, Deutschland, ${ }^{2}$ Rückenzentrum Am Michel, Praxis für Orthopädie, Hamburg, Deutschland, ${ }^{3}$ Rückenzentrum Am Michel, Praxis für Physiotherapie, Hamburg, Deutschland

Beim chronischen Schmerz handelt es sich um ein multidimensionales Phänomen mit biologisch-somatischen, kognitiv-emotionalen und behavioralen Aspekten. Im Rahmen einer multimodalen interdisziplinären Diagnostik werden auf dieser Grundlage für Patienten entsprechende befundgerechte Therapieprogramme erstellt. Nur eine gemeinsame Berücksichtigung aller Dimensionen führt zu einer angemessenen Erfassung und Behandlung des Schmerzproblems. Monodisziplinäre Diagnostik- und Therapiesettings werden den komplexen Befundmustern von Patienten mit chronischen Rückenschmerzen oft nicht gerecht (siehe Teil 1 des Workshops). Stattdessen sollte gemäß der multidimensionalen Symptomatik im Rahmen der Therapie interdisziplinär, multimodal und befundgerecht vorgegangen werden. Im Vordergrund stehen sowohl die Festlegung des schmerzverursachenden Gewebes (myofaszial, neuromeningial, artikulär), als auch die Differenzierung der schmerzerhaltenden psychosozialen Faktoren, wie z. B. typisches Angst- Vermeidungsverhalten, individuelle Bewältigungsstrategien, Motivation und Wille zur Veränderung sowie die kritische Bewertung der bildgebenden Befunde. Indizierte passive Therapieformen können aktive Therapien unterstützen, sind jedoch monomodal angewandt ggf. sogar kontraproduktiv (siehe Teil 2 des Workshops).
Die Interdisziplinäre Multimodale Schmerztherapie (MMST) bezeichnet die gleichzeitige, inhaltlich, zeitlich und in der Vorgehensweise aufeinander abgestimmte umfassende Behandlung von Patienten mit chronischen Schmerzsyndromen, in die verschiedene somatische, körperlich übende, psychologisch übende und psychotherapeutische Verfahren nach vorgegebenem Behandlungsplan mit identischen, unter den Therapeuten abgesprochenen Therapiezielen, eingebunden sind.

Die gemeinsamen Behandlungsziele lauten:

- Stärkung der funktionellen Leistungsfähigkeit und alltagstaugliche Rückenbelastbarkeit

- Wiederherstellung der Arbeitsfähigkeit und soziale Reintegration

- Verminderung der Inanspruchnahme unnützer therapeutischer und diagnostischer Maßnahmen (Bildgebung, Spritze, Operation, etc.)

- Vermittlung eines auf Schmerzkontrolle gerichteten Krankheitsmodells

- ggf. Linderung der Schmerzen

- Ablegen der Krankenrolle

- Verbesserung der Eigenmotivation

- Herstellen vermehrter Autonomie

- Verbesserung von Lebensqualität und Leistungsfähigkeit

- Reduktion von Bewegungsangst

- Veränderung psychologischer Risikofaktoren wie z.B. Schon-/Durchhalteverhalten und inadäquater Umgang mit Schmerzen

Das therapeutische Team besteht aus Ärzten bzw. Schmerztherapeuten einer oder mehrerer Fachrichtungen, Psychologen bzw. Psychotherapeuten mit Schwerpunkt Verhaltenstherapie und Schmerzpsychotherapie, Physiotherapeuten und Sporttherapeuten. Die Behandlung wird in Kleingruppen von maximal acht Patienten durchgeführt. Das Programm erstreckt sich über einen Zeitraum von mehreren Wochen.

Der Workshop besteht aus zwei Teilen (Teil 1 Diagnostik, Teil 2 Therapie). Es werden die unterschiedlichen Behandlungsbausteine einer ambulanten Intensivtherapie von den beteiligten Disziplinen ausführlich und sehr praktisch anschaulich unter Verwendung von Fallbeispielen und mit Demonstration von Behandlungselementen dargestellt.

\section{Literatur}

1. Arnold B, Brinkschmidt, T et al (2014) Multimodale Schmerztherapie für die Behandlung chronischer Schmerzsyndrome: Ein Konsensuspapier der Ad-hocKommission Multimodale interdisziplinäre Schmerztherapie der Deutschen Schmerzgesellschaft zu den Behandlungsinhalten. Schmerz 28(5):459-472

2. Casser HR, Arnold B, Brinkschmidt T et al (2013) Interdisziplinäres Assessment für die multimodale Schmerztherapie: Indikationen und Leistungsumfang. Schmerz 27(4):363-370

3. Arnold B, Brinkschmidt T, Casser HR et al (2009) Multimodale Schmerztherapie Konzepte und Indikationen. Schmerz 23:112-120

4. Lang E, Eisele R, Kastner S, Liebig K, Martus P, Neundörfer B (2000) Ergebnisqualität der ambulanten Versorgung von Patienten mit chronischen Rückenschmerzen. Schmerz 14:146-159

5. Nagel B, Pfingsten M, Brinkschmidt T et al (2012) Struktur- und Prozessqualität multimodaler Schmerztherapie - Ergebnisse einer Befragung von schmerztherapeutischen Einrichtungen. Schmerz 26(6):661-669

6. http://www.leitlinien.de/nvl/kreuzschmerz. Zugegriffen: 14.12.2015

\section{WS08 - Training interpersoneller Verhaltens- und} Steuerungskompetenz CBASP (Cognitive Behavioral Analysis System of Psychotherapy) für Patienten mit chronischem Schmerz

\section{l. Liwowsky}

Schmerzzentrum am Starnberger See, Psychologie, Tutzing, Deutschland

Ein beträchtlicher Teil chronischer Schmerzpatienten weist eine frühe Traumatisierung auf [1]. Diese frühen Missbrauchserlebnisse werden als Risikofaktor für die Entstehung und Aufrechterhaltung von chronischen Schmerzen diskutiert [2]. Die Komorbidität von chronischem Schmerz mit Depression und auch chronischer Depression ist hoch [3]. Langjährig chronifizierte Schmerzpatienten zeigen oft eine veränderte Interaktionsgestaltung. Ähnlich wie chronisch depressive Patienten sind sie sich der Effekte des eigenen Verhaltens auf das Gegenüber nicht bewusst und erleben sich als interpersonell unwirksam. Die Unfähigkeit interpersonelle Probleme zu lösen und zielführend zu handeln, führt zu starker psychi- 
scher Belastung und oft zu Resignation. CBASP ist ein von Prof. James McCullough [4] entwickeltes Behandlungsverfahren. Das zugrundeliegende Ätiologiemodell fokussiert auf einen Zusammenhang zwischen frühen Missbrauchserfahrungen und einer hieraus resultierenden Störung der Person-Umweltinteraktion. Es kommt zu Defiziten der kognitiven, emotionalen und sozialen Fertigkeiten, so dass Entwicklungsaufgaben und Lebensbelastungen nicht adäquat bewältigt werden können. CBASP fokussiert einerseits auf die Vermittlung der Situationsanalyse und ein sich daran anschließendes Verhaltenstraining, in welchem das Zielverhalten in Rollenspielen eingeübt wird. Die Situationsanalyse fördert die mentale Fähigkeit formal-operatorisch zu denken und zu handeln und hilft dem Patienten eine kausale Beziehung zwischen seinen Verhaltens- und Denkmustern und den Konsequenzen seines Verhaltens herzustellen. Wichtiges Element ist hierbei die Einordnung des eigenen Verhaltens im Kiesler-Kreis-Modell. In seinen Einzelschritten stärkt dieses Training darüber hinaus die perzeptiven und interpretativen Leistungen, die Fähigkeit zur Reflexion des eigenen verbalen und nonverbalen Verhaltens, sowie den Erwerb von Steuerungskompetenz durch die Kontrastierung des habituellen dysfunktionalen Verhaltens gegen das gewünschte Zielverhalten. Ein zweiter Schwerpunkt der CBASP-Therapie liegt auf der adaptierten Rolle des Therapeuten. Um korrigierende Beziehungserfahrungen zu fördern, werden frühe Prägungen erhoben und sich daraus ergebende Übertragungshypothesen formuliert. Der Therapeut bringt sich auf disziplinierte, verantwortungsvolle Weise persönlich (Disciplined Personal Involvement) in die therapeutische Beziehung ein, um so die Patienten zu befähigen, zwischen alten Beziehungserwartungen und dem Verhalten des Therapeuten bzw. anderer Personen zu unterscheiden (Interpersonal Discrimination Exercise). Im Workshop werden alle wesentlichen CBASP Elemente vorgestellt. Der Fokus liegt dabei auf dem Training der Verhaltens- und Steuerungskompetenz mit Hilfe des Kiesler-Kreis-Modells und den speziellen Situationsanalysen.

Referentin: Dr. rer. med., Dr. phil., Dipl. Psych., Dipl. Soz. Päd. Iris Liwowsky Leitende Psychologin im multimodalen Schmerzzentrum am Starnberger See. Psychologische Psychotherapeutin, Schmerzpsychotherapeutin, Pessotherapeutin, Supervisorin und Dozentin an der Ludwig-MaximilianUniversität und an verschiedenen Ausbildungsinstituten. Weiterbildungen zum Thema CBASP in Virginia (J. McCullough), Freiburg (E. Schramm) und München (E. Brakemeier). Mitglied im CBASP Netzwerk. Zentrum für Anästhesiologie, Intensivmedizin, Schmerztherapie \& Palliativmedizin Benedictus Krankenhaus Tutzing GmbH \& Co. KG Bahnhofstraße 5 82327 Tutzing Tel.: +49 8158-23714 Fax.: +49 8158-23711 i.liwowsky@ krankenhaus-tutzing.de Es bestehen keine Interessenskonflikte.

\section{Literatur}

1. Leisner S, Gerhardt A, Tesarz J, Janke S, Seidler GH, Eich W (2014) Frühe Missbrauchserlebnisse bei chronischem Kreuzschmerz. Direkte und mediierte Effekte früher Missbrauchserlebnisse auf verschiedene Schmerzdimensionen chronischer nichtspezifischer Kreuzschmerzen. Schmerz 28(6): 600-606

2. Lampe A, Doering S, Rumpold G, Solder E, et al (2003). Chronic pain syndromes and their relation to childhood abuse and stressful life events. Journal of Psychosomatic Research 54(4):361-367

3. Schmahl C, Bär KJ (2011) Psychopathologie und Schmerz. In: Kröner-Herwig B. Frettlöh J, Klinger R, Nilges P (Hrsg) Schmerzpsychotherapie, S. 145-152. Springer, Berlin

4. McCullough JP (2000) Treatment for Chronic Depression. Cognitive Behavioral Analysis System of Psychotherapy. Guilford Press, New York

\section{WS09 - Schmerzdiagnostik mit Skalen und Fragebögen}

P. Nilges

Johannes Gutenberg-Universität, Psychologisches Institut, Mainz, Deutschland

Zur Anwendung von Fragebögen und Skalen in der Schmerzdiagnostik bemerkt Williams:

„Die Verwendung zuverlässiger, valider und sinnvoller Verfahren ist keineswegs schwieriger als die Anwendung uninterpretierbarer oder ungeeigneter Methoden" [3].

Die Erfassung von Schmerzmerkmalen wie Intensität, Dauer, Maximum, Minimum und Qualität ist inzwischen weitgehend diagnostischer Standard. Die verwendeten Skalenformen, -formate und Instruktionen variieren dagegen noch immer erheblich.

Themen des Praktikerseminars sind Grundlagen, Auswahl und Anwendung der Verfahren im klinischen Alltag. Kriterien für „gute“ und „schlechte" Verfahren werden diskutiert.

Besprochen und praxisnah vermittelt werden die derzeit üblicherweise verwendeten Verfahren zur Schmerzmessung (VAS, NRS, Schmerztagebücher, Fragebögen zur Schmerzqualität), Verfahren zur Bestimmung der Chronifizierung (MPSS, Graduierung nach von Korrff) sowie bereichsspezifische Instrumente zur Erfassung psychischer Belastungen (depressive Symptome, Angst, Stress).

Die Auswertung und Interpretation werden praxisgerecht erarbeitet.

Dabei werden häufige Fehlerquellen, Probleme (z. B. Auswertung bei fehlenden Werten) und Entscheidungen für oder gegen bestimmte Formate sowie die Anwendungsmöglichkeiten und -grenzen beim Einsatz von Fragebögen bei Patienten mit körperlichen Beschwerden erläutert.

Vorgestellt werden die Änderungen im Schmerzfragebogen der Deutschen Schmerzgesellschaft und die neu eingeführten bzw. erweiterten Verfahren. Mit 21 Items ist die Depressions-, Angst- und Stress-Skala (DASS [1]) ein reliables, valides und gleichzeitig ökonomischer Fragebogen für Patienten mit chronischen Schmerzen.

Besonderen Stellenwert hat in diesem Praktikerseminar das Gespräch mit Patienten: Bei der Einführung der Verfahren, der Beantwortung von Fragen und Zweifeln und bei der Vermittlung der Ergebnisse.

\section{Literatur}

1. Lovibond PF, Lovibond SH (1995) The structure of negative emotional states: comparison of the Depression Anxiety Stress Scales (DASS) with the Beck Depression and Anxiety Inventories. Behavioral Research Therapy 33:335-343

2. Nilges $P$ (2013). Klinische Schmerzmessung. In: Baron R, Koppert W, Strumpf M, Willweber-Strumpf A (Eds.). Praktische Schmerzmedizin, S 79-85. Springer, Heidelberg

3. Williams AC (1995). Pain measurement in chronic pain management. Pain Reviews 2:39-63

\section{WS10 - Neurologischer Untersuchungskurs für Schmerztherapeuten: Praktische Übungen, Tricks und Syndromlehre}

\section{S. Förderreuther}

Neurologische Klinik, LMU, Konsildienst, München, Deutschland

Neurologie gilt als kompliziert und die Untersuchungstechnik als aufwendig. Wie verschafft man sich als Nicht-Neurologe einen Überblick über den neurologischen Status? Wie wird aus dem Befund erst ein Syndrom und dann sogar eine Diagnose? Welche Befunde sollten zu eingehenden neurologischen Diagnostik Anlass geben?

Im Kurs wird die neurologische Untersuchung praktisch geübt. Dabei werden nicht nur die Grundlagen der neurologischen Untersuchungstechnik vermittelt, sondern auch die neurologische Syndromlehre unter besonderer Berücksichtigung algesiologischer Krankheitsbilder. An Fallbeispielen werden interaktiv typische Befundkonstellationen und Fallstricke bei Kopfschmerzerkrankungen, zentralen und peripheren neuropathischen Schmerzsyndromen und neurologische Nebenwirkungen der Therapie vorgestellt. Die Befunde werden unter Berücksichtigung der neurologischtopischen Diagnostik besprochen. 


\section{WS12 - Umlenken erwünscht: Typische Fehldiagnosen und Möglichkeiten der interdisziplinären Aufklärung}

\section{Gaubitz' , M. Schilgen ${ }^{2}$, A. Frese ${ }^{3}$}

${ }^{1}$ Akademie für manuelle Medizin an der WWU Münster, Interdisziplinäre Diagnostik und Therapie, Münster, Deutschland, ${ }^{2}$ Akademie für Manuelle Medizin an der Westfälischen Wilhelms Universität Münster, Münster, Deutschland, ${ }^{3}$ Universitätsklinikum Münster, Neurologie, Münster, Deutschland

Hinter häufig diagnostizierten Schmerzsyndromen verbergen sich manchmal seltene, teils akut behandlungsbedürftige Entitäten. Die interdisziplinäre Untersuchung und Beratung beschleunigt oder ermöglicht erst die richtige Diagnose.

Im Symposium sollen seltene und wichtige Differentialdiagnosen häufiger Schmerzbilder mit den Gliederungspunkten Fallvorstellung, typische Symptome, red flags und Therapieckpunkte vorgestellt werden. Nach derzeitigem Stand sind folgende Fälle vorgesehen:

- Schulter-Arm-Schmerz mit V.a. Bandscheibenvorfall: Endgültige Diagnose: Neuralgische Schulteramyotrophie

- Fingergelenkschmerzen mit V.a. Fingergelenkspolyarthrose: Endgültige Diagnose: Psoriasisarthritis

- Schulter-Arm-Syndrom bei Überlastung: Endgültige Diagnose: Polymyalgia rheumatica

- Schulter-Nacken-Schmerz: Endgültige Diagnose: Dissektion der Halsarterien

- Schulterhartspann mit pulssynchronem Ohrgeräusch: Endgültige Diagnose: Polymyalgia rheumatika mit zerebraler Vaskulitis und A.basilaris-Stenose

Vorgestellt werden die Fallberichte und Krankheitsvignetten aus der täglichen interdisziplinären Arbeit von den Mitgliedern der Praxisgemeinschaft „Interdisplinäre Diagnostik und Therapie“ in der Akademie für Manuelle Medizin Münster an der WWU Münster:

- Prof. Dr. Markus Schilgen, Orthopäde, Spezielle Schmerztherapie, Manuelle Medizin

- Priv-Doz. Dr. Achim Frese, Neurologe, Spezielle Schmerztherapie

- Prof. Dr. Markus Gaubitz, Internist, Rheumatologe

Freitag, 21.10.2016

\section{WS13 - Achtsamkeit und Akzeptanz in der Schmerztherapie \\ J. Korb \\ DRK Schmerz-Zentrum, Tagesklinik, Mainz, Deutschland}

Kognitiv-verhaltenstherapeutische Verfahren mit dem Ziel, die Selbstwirksamkeit des Patienten zu erhöhen, haben sich als sehr effizient erwiesen. Andererseits zeigt sich in vielen Studien, dass auch eine Haltung der Schmerzakzeptanz mit verringerter körperlicher und psychischer Beeinträchtigung einhergeht. In diesem Zusammenhang wird oft eingewandt, dass wiederholte Versuche der Schmerzbeeinflussung und Kontrolle die Aufmerksamkeit des Patienten verstärkt auf die Schmerzthematik lenken und damit wesentliche Energie von anderen wichtigen Lebensbereichen abziehen können.

Doch wie lässt sich die protektive Wirkung der Schmerzakzeptanz therapeutisch vermitteln, ohne dabei Widerstände beim Patienten auszulösen („Ich will nicht lernen, mit den Schmerzen zu leben, ich will ohne Schmerzen leben!')? Bereits 1985 hat Jon Kabat-Zinn seine Therapie der „mindfulness-based stress reduction" (MBSR) auf chronische Schmerzpatienten angewandt. Ein jüngerer Ansatz kommt aus der Richtung der Akzeptanzund Commitment-Therapie (Hayes et al. 2004), der inzwischen auch für die Behandlung chronischer Schmerzpatienten konkretisiert wurde (McCracken 2005; Dahl et al. 2005).

Vor allem dieser neuere Therapieansatz soll kurz vorgestellt werden. Anhand praktischer Beispiele werden Interventionen zur Förderung einer
Haltung der Akzeptanz und Übungen zur Achtsamkeit gezeigt, jedoch auch Schwierigkeiten und Grenzen der Verfahren diskutiert werden.

\section{Literatur}

1. Dahl J, Wilson KG, Luciano C (2005) Acceptance and commitment therapy for chronic pain. Context Press

2. Hayes SC, Strohsahl KD, Wilson KG (2004) Akzeptanz und commitment therapie. Cip-Medien

3. Kabat-Zinn (1985) The clinical use of mindfulness meditation for the self-regulation of chronic pain. Journal of Behavioral Medicine 8:163-190

4. McCracken LM (2005) Contextual-behavioral therapy for chronic pain. Intl Assn for the Study of Pain

\section{WS14 - Neuroorthopädische und funktionelle Untersuchung für Schmerzmediziner}

\section{K. Niemier ${ }^{1}$}

${ }^{1}$ Klinik für Manuelle Therapie, Hamm, Deutschland

Die Untersuchung von Patienten mit Rückenschmerzen dient verschiedenen Zielen. Sie stellt Kontakt mit dem Patienten her (Beziehungsarbeit), sie gibt Information zu degenerativen Veränderungen der Wirbelsäule und Gelenken und ist somit Voraussetzung für eine gezielte apparative Diagnostik, sie gibt Informationen über den Funktionszustand der Muskulatur, Gelenke und anderer Gewebe und ist damit Grundlage für die Durchführung funktioneller Behandlungen (z. B. Manueller Medizin) bzw. der Verschreibung von Physiotherapie und sie kann Diskrepanzen zwischen Schmerzerleben und körperlichen Befund deutlich machen als Grundlage für die Erarbeitung eines ganzheitlichen Krankheitsverständnisses. Im Therapieverlauf können Erfolge und Misserfolge anhand der Veränderung oder Nichtveränderung insbesondere der funktionellen Befunde besser beurteilt und die Therapie angepasst werden.

In diesem Praktikerseminar soll eine Basisuntersuchung mit einem Schwerpunkt auf die funktionellen Befunde vorgestellt werden. Primäre Funktionsstörungen wie die mangelnde Stabilisation der Wirbelsäule und der Gelenke, Koordinationsstörungen und die konstitutionelle Hypermobilität werden systematisch gesucht um Rezidive von sekundären (schmerzhaften) Funktionsstörungen wie z. B. Blockierungen und Triggerpunkten zu verhindern. Weiterhin soll der Zusammenhang zur funktionellen Behandlung und der Verschreibung von Physiotherapie erläutert werden.

\section{WS15 - Naturheilkunde in der Schmerztherapie}

\section{Werner}

Kliniken Essen-Mitte, Klinik für Naturheilkunde und Integrative Medizin; Kliniken Essen Mitte, Medizinische Fakultät, Universität Duisburg-Essen, Essen, Deutschland

Naturheilkundliche Therapieverfahren werden zunehmend von Schmerzpatienten nachgefragt. Die Kenntnis von sinnvoll angewendeten Verfahren kann die Patientenbindung verbessern und bei therapierefraktären Verläufen neue Optionen bieten. Auch können Nebenwirkungen oder das Vorliegen von schweren Begleiterkrankungen konsequente Umsetzung der konventionellen Verfahren beeinträchtigen. Insgesamt sind naturheilkundliche Therapien bei chronischen Schmerzen wirksame, meist kostengünstige und nebenwirkungsarme Therapieoptionen. Sie können oft schnell und effektiv in den Praxisalltag integriert werden. In vielen Fällen kann durch die Kombination mit nicht-medikamentösen naturheilkundlichen Verfahren eine Schmerzmittelreduktion oder ein komplettes Absetzen der Schmerzmedikation bei gesteigerter Lebensqualität erreicht werden.

In diesem Workshop werden wesentlichen Behandlungsmodule für eine erfolgreiche integrative, multimodale Schmerztherapie vorgestellt. Weiterhin werden ausleitende Verfahren (Blutegeltherapie, Schröpfen und Co.) dargestellt. Im letzten Teil des Workshops werden Selbsthilfestrategien für Schmerzpatienten praktisch präsentiert, die sich lohnen, und die die Patienten nachhaltig nutzen. 
WS16 - Strategien in der postoperativen Schmerztherapie anhand von Fallbeispielen

\section{K. Ferlemann}

Universitätsmedizin Göttingen, Schmerzklinik, Göttingen, Deutschland

In diesem Workshop sollen beispielhafte Kasuistiken interessanter postoperativer Problemfälle vorgestellt werden. Die Fälle sind real und resultieren aus der konsiliarischen Betreuung einer schmerztherapeutischen Einrichtung in einem großen Universitätsklinikum.

Thematisch handelt es sich um Patienten mit vorbestehendem Opiatabusus, junge Menschen mit langer Krankenhauskarriere, Patienten mit postoperativer Opiatgewöhnung, multimorbide Patienten und CRPS Patienten.

Die Fälle werden ca. 5 min in ihren Spezifika vorgestellt, danach interaktiv mit den Teilnehmern potentielle Lösungswege erarbeitet und zum Schluss die durch uns gewählte Vorgehensweise mit dem erzielten Resultat vorgestellt.

\section{WS17 - Mindfulness-Based Stress Reduction (MBSR nach Kabat- Zinn) in der Therapie chronischer Schmerzen - eine Einführung mit praktischen Übungen}

H. Lucius $^{1}$

'Schmerzentrum NORD am HELIOS-Klinikum Schleswig, Schmerzambulanz der Fachklinik, Schleswig, Deutschland

1979 gründete der Amerikaner Jon Kabat-Zinn die Stress Reduction Clinic, an der er in den folgenden Jahren das Programm der MindfulnessBased Stress Reduction/MBSR (zu dt. achtsamkeitsbasierte Stressreduktion) entwickelte und an seine Schüler/Innen weitergab. Das Training erfolgte zunächst mit chronisch Schmerzkranken und Krebspatienten. Im Laufe der Zeit wurde die "Methode“ dann an viele Praktizierende in Form eines standardisierten Acht-Wochen-Kurses weltweit weitergegeben. Seit mehr als 10 Jahren wird MBSR u. a. in Form von 8-Wochenkursen und Jahrestrainings an verschiedenen Orten in Deutschland angeboten, zunehmend auch in Unternehmen, Behörden und Schulen. Ein Forschungsschwerpunkt besteht in Freiburg, ein weiteres Zentrum befindet sich im Klinikum Essen Mitte in der Klinik für Naturheilkunde und Integrative Medizin. Hinzu kommen viele Einrichtungen, in denen auch auf dem Gebiet der spirituellen Wissenschaften über MBSR geforscht wird. Das Interesse der Neurobiologie, insbesondere auch mittels bildgebender Verfahren Meditation und MBSR zu erforschen, ist hoch. Z. B. laufen am Bender Institute of Neuroimaging in Gießen ständig Untersuchungen zu Fragen der Meditation. In der Laienpresse und der öffentlichen Diskussion nimmt das Thema Achtsamkeit (z. B. am Arbeitsplatz) einen immer breiteren Raum ein, nicht unbedingt zum Vorteil von MBSR

Zur Effektivität in der Schmerztherapie existieren inzwischen zahlreiche (auch kontrollierte) Studien über verschiedene Störungen vor wie dem Fibromyalgiesyndrom (Schmidt,S., et al; PAIN 152/2011), chronische Kopf-, und Rückenschmerzen und Migräne (Simshäuser, K.), Daten aus der Onkologie und der Palliativmedizin liegen ebenfalls vor. Die Effektstärke nach Cohen ist in einer älteren Metaanalyse zu MBSR von Grossmann et al. (2004) mit 0,5 erfasst, eine neuere Studie von Kohls et al (2011) zeigte eine solche von 0,6. Untersuchungen zur Auswirkung des Trainings in Unternehmen oder Schulen sind nur spärlich vorhanden. An der LMU München und im Institut für spirituelle Wissenschaft in Coburg wird über diese Themen geforscht.

Was Achtsamkeit nicht ist: Achtsamkeitsübungen nach MBSR sind nicht etwa ein "neues Konzept", es handelt sich nicht um eine Therapie im engeren Sinne, es ist frei von keine religiös-spiritueller Anbindung und MBSR hat nicht primär etwas mit der Lehre des Buddhismus zu tun. Achtsamkeit ist auch eigentlich keine Methode.

Was Achtsamkeit ist: Achtsamkeit ist aber eine (geistige) Einstellung und Haltung, in der man sich um ein breites und gleichmütiges An- und Wahrnehmen aller sich einstellenden Phänomene bemüht. Dies bedeutet, alle entstehenden und vergehenden Erscheinungen (im Geist) mit einer gelassenen Akzeptanz zu „betrachten“, ohne sie verändern, beeinflussen oder auch loslassen zu wollen - es gibt im Rahmen dieses Prozesses kein richtig oder falsch. Regelmäßiges Üben ermöglicht eine offene Präsenz und im Optimalfall ein beständiges Aufmerksamsein nach dem Grundsatz: Ein Buddha ist ein Mensch, der 24 Stunden am Tag in Achtsamkeit lebt (Tich Nhat Hanh).

Ergänzend sei erwähnt, dass Achtsamkeit und Akzeptanz im Rahmen der sog. Mind-Body-Medicine, der Dialektisch-Behavioralen Therapie (DBT) eine wichtige Rolle spielen. In Ergänzung zu MBSR entstand die Mindfulness-Based Cognitive Therapy (MBCT) nach Teasdale et al. als Methode zur Unterstützung der therapeutischen Arbeit. Außerdem sind Bestandteile der Acceptance- and Commitment-Therapy (ACT) nach Hayes et al. Teil psychotherapeutischer Angebote. Eine Fülle von MindfulnessBased ... .Prozeduren wird angeboten zur Behandlung von Suchterkrankungen, Essstörungen, $\mathrm{AD}(\mathrm{H}) \mathrm{S}$ und anderen. Die formale Praxis des Trainings besteht aus:

1. Dem Body Scan, einer achtsamen Körperwahrnehmung

2. Einer Sitzmeditation (gern durch achtsames Gehen ergänzt)

3. Achtsamen Körperübungen, die meist der Tradition des Yoga entstammen.

Eine wichtige Rolle spielt die Alltagspraxis (achtsames Essen, Haare waschen), achtsame Kommunikation wird geübt und immer wieder sind Gedanken und Gefühle wahrzunehmen ohne diese zu bewerten.

Der Workshop wird in die Thematik der Meditation im Rahmen von MBSR anhand eines Vortrags mit Übungen einführen. Der Nutzen eines solchen kurzen „Trainings“ besteht für die Teilnehmer/Innen im Kennenlernen einer effektiven Methode zur Geistesschulung in der eigenen Burnout- und Stressprophylaxe - Voraussetzung für erfolgreiche Beziehungen und ein tragfähiges Arzt-Patienten-Bündnis (Schulz-von Thun, Watzlawik) in der Therapie chronischer Schmerzen. In diesem Sinne lässt sich auch eine achtsame Unternehmenskultur entwickeln und fördern. Darüber hinaus wird sich die eigene Lebensqualität bei regelmäßiger Übung deutlich steigern.

„Die Schönheit der meditativen Arbeit liegt darin, dass wir auf die Praxis selbst vertrauen können, um uns aus dem Schlamassel zu führen. Sie hält uns auf dem Pfad, selbst in den dunkelsten Momenten - sie stellt sich selbst unseren fürchterlichsten Geisteszuständen und äußerlichen Bedingungen. Sie erinnert uns an unsere Möglichkeiten." (Jon Kabat-Zinn)

\section{Literatur}

Kabat-Zinn J (1998) Im Alltag Ruhe finden. Herder Verlag Freiburg, Freiburg Kabat-Zinn J (2006) Gesund durch Meditation. Fischer Verlag. Frankfurt/Main Ott U (2010) Meditation für Skeptiker. Verlag O.W. Barth, München

Santorelli S (2006) Zerbrochen und doch ganz. Die heilende Kraft der Achtsamkeit. Arbor Verlag Freiamt, Freiburg

Malinowski P (2010) Flourishing. Welches Glück hätten Sie gern? Irisana Verlag, Haldenwang

Burch V (2009) Gut leben trotz Schmerz und Krankheit. Goldmann/Arkane Verlag, München

Esch T (2013) Die Neurobiologie des Glücks. Thieme Verlag, Stuttgart

Song $Y$ et al (2014) Mindfulness intervention in the management of chronic pain and psychological comorbidity: A meta-analysis. Nursing Sciences

Grossmann P et al (2004) Mindfulness-based stress reduction and health benefits: A meta-analysis. JPsychos Res 57:35-43

\section{WS18 - Klinische Differenzierung und gezielte Behandlung des Schulter-Nacken-Schmerzes}

H. Casser', M. Graf ${ }^{2}$

'DRK Schmerz-Zentrum Mainz, Mainz, Deutschland, ${ }^{2}$ Praxis, Trier, Deutschland

Themen: Differentialdiagnostik des Schulter- Nackenschmerzes (Demonstration eines klinischen Untersuchungs-Algorithmus) Hans-Raimund Casser (Mainz) Befundorientierte therapeutische Maßnahmen (mit Demonstration) Michael Graf (Trier) Inhaltsbeschreibung: Schulter-Nacken-Schmerzen stellen eine differentialdiagnostische Herausforderung dar. Spezielle Erkrankung des Schultergelenkes wie das Subacromial-Syndrom als Ausdruck einer Bursitis subacromialis, einer Rotatorenmanschetten- oder Bizepssehnenruptur oder einer Tendinosis calcarea, aber 
auch Beschwerden aufgrund einer Omarthrose oder einer rheumatischen Synovitis des scapulothorakalen Gelenkes bis hin zur Acromioclavikulargelenksarthrose lösen chrarakteristische Beschwerden bzw. Funktionsstörungen auf, die in erster Linie durch die klinische Untersuchung zu differenzieren sind. Davon abzugrenzen sind die neuralgische Schulteramyotrophie, die Capsulitis adhaesiva („Frozen Shoulder“), Radikulopathien der unteren Halswirbelsäule (C5-8) und das Quadrantensyndrom als Ausdruck einer vegetativen Schmerzsymptomatik bis hin zu myofasciellen Schmerzsyndromen der Schulter- Nackenmuskulatur mit Triggerpunkten vorzugsweise im Trapezius, Sternocleidomastoideus oder den Scaleni, die ebenfalls eine Domäne der klinischen Untersuchung, orthopädisch-manualdiagnostisch sowie neurologisch-elektrophysiologisch, sind. Differentialdiagnostisch müssen auch Schulterschmerzen neurologischer Genese berücksichtigt werden, die zur Desintegration der Schulter-Nacken-ArmRegion führen. Die Läsion nozizeptiver Fasern dagegen kann ein neuropathisches Schmerzsyndrom auslösen. Häufig liegen nozizeptive und neuropathische Schmerzkomponenten gemeinsam vor. Einen weiteren Aspekt stellen sympathisch unterhaltene Schmerzen dar. Im Rahmen chronischer Beschwerdebilder spielen psychische Faktoren bei Entstehung und Unterhaltung der Schulter-Nacken-Schmerzen eine zunehmende Rolle. Sie müssen frühzeitig erfasst und ggf. schmerzpsychologisch in einem multimodalen interdisziplinären Therapieprogramm ausreichend berücksichtigt werden. Unter Einbeziehung orthopädischer, manualdiagnostischer, neurologischer und schmerzpsychologischer Aspekte soll ein praxisorientiertes Konzept aufgezeigt werden, um relevante anamnestische und klinische Zeichen einschließlich bildgebender Befunde zu erkennen, kompetent $\mathrm{zu}$ interpretieren und für das therapeutische Vorgehen entsprechend zu verwerten.

\section{Kopfschmerzversorgung in Deutschland - Quo vadis?}

\section{U. Meier' ${ }^{1}$ A. Straube ${ }^{2}$, M. Marziniak ${ }^{3}$}

'Praxisklinik Grevenbroich, Grevenbroich, Deutschland, ${ }^{2}$ Klinikum Großhadern, Neurologische Klinik und Poliklinik, München, Deutschland, ${ }^{3}$ Isar-Amper-Klinikum, Klinik für Neurologie, Haar, Deutschland

U. Meier: Die Rolle des Schmerzes und des Kopfschmerzes in der ambulanten Versorgung: Versorgungsstrukturen aus der Sicht der Berufsverbände und Erfahrungen in der Integrierten Versorgung Kopfschmerz Nordrhein

A. Straube: Die Integrierte Versorgung am Beispiel des oberbayrischen Kopfschmerzzentrums

M. Marziniak: Aktuelle Versorgung von Patienten mit Kopfschmerzen und Vorstellung der Zertifizierung von Kopfschmerzzentren

Das Symposium möchte einen Überblick geben über die aktuelle Kopfschmerzversorgung in Deutschland und die weiteren Möglichkeiten der Verbesserung diskutieren. Insbesondere soll ein Einblick in zwei Bereiche der integrierten Versorgung Kopfschmerz gegeben werden.

Herr Dr. Meier wird als 2. Vorsitzender des BDN und zuständiger Referatsleiter des BVDNs: Schmerz in der Neurologie sowie Mitglied im Beirat Integrierte Versorgung Kopfschmerz Nordrhein einen Überblick geben über die Versorgung und die Aufgaben der Versorgung von Schmerzpatienten unter besonderer Berücksichtigung von Kopfschmerzen und seine Erfahrungen aus der integrierten Versorgung Kopfschmerz Nordrhein einfließen lassen.

Herr Prof. Straube wird die Möglichkeiten der Verbesserung der Versorgung durch die integrierte Versorgung Kopfschmerz (IGV) des oberbayrischen Kopfschmerzzentrums vorstellen. Ein wesentlicher Baustein bei der IGV ist, dass genug Zeit für eine Psycho-Edukation eingeräumt werden kann, dieses kann gerade durch eine 5-tägigen Tag-Klinik Aufenthalt noch intensiviert werden und führt zu einer auch nach $1 \mathrm{Jahr}$ noch nachweisbaren signifikanten Verbesserung der Schmerzbelastung.

Herr Prof. Marziniak wird die aktuelle Versorgungslage der Patienten mit Kopfschmerzen in Deutschland vorstellen und auch die hohe Selbstmedikationsrate bei Kopfschmerzen diskutieren. Im zweiten Teil des Vortrags ist die Vorstellung der Zertifizierung der Kopfschmerzzentren der DMKG geplant (Marziniak et al., 2014).

\section{WS20 - Schmerz bei älteren Menschen mit kognitiven Einschränkungen}

\section{Schuler', M. Laekeman²}

'Diakonissenkrankenhaus, Akutgeriatrie, Mannheim, Deutschland, ${ }^{2}$ Universität Witten/Herdecke, Fakultät für Gesundheit, Bochum, Deutschland

Das Thema „Schmerz bei älteren Menschen“ gewinnt in den letzten Jahren mehr und mehr an Bedeutung. Akute und chronische Erkrankungen wie auch der Schmerz selbst können zu kognitiven Beeinträchtigungen (Verschlechterung einer bekannten dementiellen Erkrankung oder zu einem Delir) führen. Für diese Probleme ist das Alter ein nicht zu beeinflussender Risikofaktor. Andere Risikofaktoren lassen sich jedoch beeinflussen, sofern sie erkannt werden. Hierzu zählen u. a. der Schmerz selbst und die Schmerztherapie. Da medikamentöse Schmerztherapie zum einen zur Schmerzlinderung und Besserung der kognitiven Funktionen führen, allerdings auch das Gegenteil bewirken kann, sollen in diesem Zusammenhang auch physiotherapeutische Möglichkeiten der Schmerzbehandlung thematisiert werden. Bei diesen Menschen ist das Schmerzmanagement vom Erkennen eines Schmerzproblems, über den Umgang mit Patient und seinen Schmerzen bis hin zur Umsetzung spezifischer Behandlungen eine Herausforderung.

Im Workshop werden zwei Bereiche angesprochen:

1) Prävention und Management von kognitiver Beeinträchtigung: Schmerz, Analgetika, Ortswechsel, fehlende Hörgeräte sind nur einige Risiken, die zur kognitiven Verschlechterung beitragen können. Welche Lösungen für diese Probleme möglich sind und auf welchem wissenschaftlichen Fundament diese beruhen, wird dargestellt. (Schuler M.)

2) Besonderheiten der Schmerzphysiotherapie bei Menschen mit Demenz:

Schmerzphysiotherapie bei diesen Menschen verlangt eine person-zentrierte Arbeitsweise. Das Einbinden der Angehörige und den Einsatz von innovativen Ideen sind hilfreiche Ressourcen. Trotzdem stehen auch Physiotherapeuten öfters vor Grenzen, die im Workshop diskutiert werden. (Laekeman M.)

\section{WS21 - Lokaltherapie neuropathischer Schmerzen}

\section{F. Birklein', C. Maihöfner' ${ }^{2}$, G. Wasner ${ }^{3}$}

'Universitätsmedizin der Johannes Gutenberg-Universität Mainz, Klinik für Neurologie, Mainz, Deutschland, ${ }^{2}$ Klinikum Fürth, Chefarzt der Neurologischen Klinik, Fürth, Deutschland, ${ }^{3}$ Neurologische Praxis Kiel, Kiel, Deutschland

Botulinum Toxin bekommt in der Schmerztherapie einen zunehmend höheren Stellenwert. Nachdem BoNT für die Therapie der chronischen Migräne offiziell zugelassen wurde, fand BoNT nach der Publikation von 3 positiven Studien bei fokal neuropathischen Schmerzen und diabetischer Neuropathie in der Zwischenzeit auch Erwähnung in Leitlinien zur Behandlung neuropathischer Schmerzen. Der Vortrag wird Therapiestudien zusammenfassen und neueste Daten zur Wirkweise von BoNT auf periphere Nozizeptoren referieren. (Birklein) Die Defunktionalisierung von TRPV1-positiven C-Fasern durch eine topische Applikation mit hochdosiertem Capsaicin ist ein zunehmend relevantes Therapieelement bei neuropathischen Schmerzen. In dem Vortrag soll auf die aktuelle Studienlage zu diesem Thema eingegangen werden, insbesondere werden Prädiktoren für das Ansprechen vorgestellt und kritisch hinterfragt. Ebenfalls werden aktuelle Konzepte und Studien bezüglich einer Kombinationstherapie (topische und systemische Therapie) vorgestellt. Schließlich wird auf pathophysiologische Grundlagen der Wirkweise dieser Therapieform eingegangen. (Maihöfner) Lokalanästhetika (LA) blockieren Na+ Kanäle am Ort ihrer Applikation. Lidocainhaltige Matrixpflaster sind in der Therapie der postherpetischen Neuralgie etabliert. in jüngsten Untersuchungen konnte aber auch gezeigt werden, dass lokal applizierte Lokalanästhetika bei vermeintlich generalisierten Schmerzerkrankungen mit Zeichen von zentraler Schmerzverstärkung (Postherniotomie; Phantomschmerz) die 
Symptome teilweise zum Verschwinden bringen. Der Vortrag wird neue Erkenntnisse der Therapie neuropathischer Schmerzen durch Applikation von LA erklären. (Wasner)

\section{WS22 - Stress \& Schmerz: Untersuchung und Biofeedback bei Kopf- und Gesichtsschmerz}

\section{Ettlin', N. Lukic', B. Steiger \\ 'Universität Zürich, Klinik für Kaufunktionsstörungen, abnehmbare Prothetik, Alters- und Behindertenzahnmedizin, Zürich, Schweiz, 2Universität Zürich, Zentrum für Zahnmedizin/Schmerzsprechstunde, Zürich, Schweiz}

Stressbelastungen können zu (unbewusster) Erhöhung des Muskeltonus der Kaumuskulatur im Sinne von Zähnepressen/-knirschen führen. Im Fachjargon wird dafür der Begriff „Parafunktion“ bzw. „Bruxismus“ verwendet. Im Zeitverlauf können bei Betroffenen Kopf-, Gesichts- und Kieferschmerzen resultieren. Diese Symptome können umgekehrt als Stressbelastung erlebt werden und zu Verunsicherung und erhöhter Anspannung führen. Insbesondere das bedrohliche Erleben von Beschwerden führt oft zu einer Fokussierung auf zuvor unwesentliche Merkmale (z. B. Zahnstellung) im Sinne einer überhöhten Körperempfindung (sog. „Hypervigilanz"). Damit gehen angstbesetze Gedanken und Befürchtungen einher. Z. B. werden Schmerzen als Zeichen einer bösartigen Erkrankung oder harmlose Kiefergelenkgeräusche als bedrohliche Gelenkschädigung interpretiert. Diese Symptomatik wird verstärkt, wenn von therapeutischer Seite ein inadäquates Krankheitsmodell vermittelt wird oder gar unnötige, vermeintlich therapeutische Eingriffe vorgeschlagen werden. Anhand von Videobeispielen aus der Klinik werden biopsychosoziale Konzepte vorgestellt. Im praktischen Teil üben Teilnehmende die klinische Untersuchung und erlernen die Anwendung von Biofeedback. Der Workshop gliedert sich in drei Teile: 1. Kopf-, Gesichts- und Kieferschmerz: Überblick zur multifaktioriellen Ätiologie und vielfältigen Symptomatik (Dominik Ettlin/Beat Steiger) 2. Praktische Übungen zur Untersuchung des Kiefergelenks und der Kaumuskulatur (Nenad Lukic/Dominik Ettlin) 3. Biofeedback: Angewandte Psychophysiologie im interdisziplinären Behandlungskonzept von Kopf-, Gesichts- und Kieferschmerzen. (Beat Steiger)

\section{WS23 - „Wenn der Kopf zerbricht" - Hypnose und Selbsthypnose bei Kopfschmerzen}

\section{A. Pielsticker \\ 'Institut für Schmerztherapie München, München, Deutschland}

Kopfschmerzen bereiten häufig Kopfzerbrechen. Gewohnte Verhaltensmuster wie das perfekte Funktionieren im Alltag können nicht mehr aufrechterhalten werden. Der während einer Migräneattacke erlebte Verlust von Kontrolle über den eigenen Körper macht hilflos und deprimiert. Hier stoßen auch bewährte Schmerzbewältigungsverfahren an ihre Grenzen Mit der Anwendung klinischer Hypnose kann auf das Leiden und die emotionalen Aspekte bei Kopfschmerzen besonders gut eingegangen werden. Hypnose kann sowohl während akuter Kopfschmerzphasen als auch in kopfschmerzfreien Intervallen zur Prophylaxe eingesetzt werden. Bei akuten Kopfschmerzen ist die Hypnose indiziert, wenn Analgetika unwirksam bleiben oder die Gefahr eines Schmerzmittelübergebrauchs besteht. In der akuten Kopfschmerzphase werden Verfahren angewandt, die eine Schmerzlinderung bewirken. Im Rahmen der Prophylaxe von erneuten Kopfschmerzattacken bei Migräne oder bei einer bereits bestehenden Chronifizierung der Kopfschmerzen können insbesondere Verfahren angewandt werden, mit denen mehr Schmerzakzeptanz und Zuversicht erreicht werden können. In der Folge entwickelt sich in der Regel eine reduzierte Grundspannung, die weniger anfällig für die Entwicklung neuer Kopfschmerzphasen macht (Pielsticker 2013).

Die Wirksamkeit klinischer Hypnose bei Kopfschmerzen konnte inzwischen gut belegt werden. Hammond (2007) kam zu dem Ergebnis, dass Hypnose im Vergleich zu Biofeedbackverfahren und konventionel- len medizinischen Behandlungen gleichwertig oder sogar überlegen ist. In vergleichenden Therapiestudien konnten durch Selbsthypnose Verringerungen der Schmerzstärke und des Medikamentenkonsums sowie Verbesserungen im Krankheitsverhalten und der Anwendung von Coping-Strategien belegt werden (Spinhoven u. ter Kuile 2000). Für die Aufrechterhaltung des Therapieerfolges scheint die Kontrollattribution (Attribution auf selbst durchgeführte Maßnahmen) eine entscheidende Bedeutung zu haben.

Auf der Basis der wissenschaftlichen Befunde werden im Rahmen des Workshops die Möglichkeiten der Hypnose in der Behandlung von Kopfschmerzen aufgezeigt und durch Einzelfalldarstellungen und eine Demonstration anschaulich präsentiert. Die vorgestellten Anwendungsbereiche beziehen sich auf die Symptomkontrolle (Veränderung der Schmerzempfindung) und den Angstabbau bei wiederkehrenden Schmerzen (Utilisieren von Prodromen bei Migräne). An praktischen Beispielen werden weiterführende Strategien zur Selbsthypnose aufgezeigt (Alman 2011).

\section{WS24 - Screening in der Physiotherapie \\ K. Lüdtke}

Universitätsklinikum Hamburg Eppendorf, Institut für Systemische Neurowissenschaften, Hamburg, Deutschland

Erkennen von Red Flags - eine Verantwortung von Physiotherapeuten? Referentin: Dr. Kerstin Lüdtke Im Verlauf physiotherapeutischer Behandlungen äußern Patienten oft Symptome, die während der ärztlichen Untersuchung keine Erwähnung fanden. Symptome können sich im Behandlungsverlauf verändern oder neu hinzukommen. Weisen diese veränderten Symptome möglicherweise auf eine bedrohliche Erkrankung hin? Welche Möglichkeiten hat der Therapeut, einen "Screeningprozess“ durchzuführen um zu entscheiden, ob das vorliegende Problem physiotherapeutisch zu behandeln ist? In kritischen Situationen ist es essentiell, effiziente und bewusste Entscheidungen zu fällen um zeitnah eine gezielte ärztliche Diagnostik einzuleiten. Ein strukturiertes Screening-Prozedere wird zur Komplementierung der physiotherapeutischen Befunderhebung vorgestellt. Die Anwendung des Prozederes wird anhand von Fallbeispielen aus der klinischen Praxis in der Gruppe geübt und diskutiert.

\section{WS25 - Diagnostische und therapeutische Lokalanästhesie myofaszialer Triggerpunkte}

U. Kern

Institut für Schmerzmedizin/Schmerzpraxis Wiesbaden, Wiesbaden, Deutschland

Myofasziale Schmerzsyndrome - gekennzeichnet durch Triggerpunkte werden in den vergangenen Jahren zunehmend erforscht und beachtet. Sie spielen im schmerztherapeutischen, klinischen Alltag eine ausgesprochen große Rolle.

Muskuläre Triggerpunkte (TP) sind überempfindliche, umschrieben tastbare Muskelareale, die typischerweise auf Kompression ausstrahlende Schmerzen in neurologisch ,nicht erklärbare' Gebiete und autonome Reaktionen erzeugen. Pathophysiologisch sind an diesen Übertragungsschmerzen offenbar Sensibilisierungen von Hinterhornneuronen mit entfernter gelegenen, rezeptiven Feldern beteiligt.

Die Palpation eines solchen Triggerpunktes führt beim Patienten zum sog. „Jump sign“, einem unwillkürlichen Wegzucken des Patienten. Klinik und Pathophysiologie von TP werden besprochen, ihre Identifikation erläutert und in Videobeispielen demonstriert. Die diagnostische und therapeutische Lokalanästhesie von Triggerpunkten ist zentraler Gegenstand dieses Workshops, periphere Nervenblockaden zur Abgrenzung neuropathischer Schmerzgenesen werden angesprochen. 


\section{Samstag, 22.10.2016}

\section{WS26 - Behandlung schmerzbezogener Angst bei Rücken- und Kopfschmerzen}

E. Hans, E. Liesering-Latta

DRK Schmerz-Zentrum Mainz, Tagesklinik, Mainz, Deutschland

Angst vor Schmerzen, körperlicher Aktivität und Schädigung bzw. Beeinträchtigung treten bei Schmerzpatienten häufig auf. Das Erleben von Angst spielt eine bedeutsame Rolle bei der schmerzbedingten Belastung, Behinderung und Schmerzchronifizierung. In diesem Zusammenhang haben sich v.a. der Denkstil des Katastrophisierens und das Angst-Vermeidungs-Verhalten (fear-avoidance) als wichtige schmerzpsychotherapeutische Ansatzpunkte erwiesen (Holroyd et al., 2007; Vlaeyen \& Linton, 2000).

Überzeugungen über den Zusammenhang zwischen körperlicher Aktivität und Rückenschmerzen, sog. Angst-Vermeidungs-Einstellungen (fear-avoidance-beliefs), führen zur angstmotivierten Vermeidung eines normalen Bewegungsverhaltens und stellen somit einen wesentlichen Risikofaktor für die Chronifizierung von Rückenschmerzen dar. Patienten mit hohen fear-avoidance-beliefs weisen eine stärkere schmerzbedingte funktionelle Beeinträchtigung auf. Die Reduzierung von fear-avoidancebeliefs ist ein guter Prädiktor für den Therapieerfolg (Leeuw et al., 2007). Bei Kopfschmerzen können Erwartungsängste bezogen auf den Schmerz, die einhergehenden Funktionseinbußen und die vermuteten Auslöser (Trigger) eine bedeutsame Rolle spielen. katastrophisierende Bewertungen können über die hierdurch ausgelöste Angst, einhergehende körperliche Stressreaktion und Aufmerksamkeitsfokussierung zu ungünstigen Verhaltensweisen (z.B. Vermeidung von Aktivitäten, zu frühe Medikamenteneinnahme) und im Sinne einer selbsterfüllenden Prophezeiung schließlich zur tatsächlichen Auslösung von Kopfschmerzen führen. Die oftmals noch propagierte Empfehlung einer generellen Vermeidung potentieller Trigger kann die Lebensqualität und den Handlungsspielraum der Betroffenen stark einschränken sowie Erwartungsängste verstärken und aufrechterhalten (Martin, 2010).

Bei der Behandlung von Schmerzpatienten ist daher die Exploration und hinreichende Berücksichtigung schmerzbezogener Ängste unerlässlich. Nach einem kurzen theoretischen Überblick und der Vorstellung verschiedener Methoden zur Erfassung von schmerzbezogenen Ängsten liegt der Schwerpunkt des Workshops auf der praxisnahen Vermittlung kognitiv-verhaltenstherapeutisch orientierter Interventionen bei Rückenund Kopfschmerzen. Anhand konkreter Fallbeispiele sollen das praktische Vorgehen veranschaulicht und Besonderheiten wie auch Schwierigkeiten bei der Durchführung diskutiert werden.

\section{Literatur}

Holroyd KA, Drew JB, Cottrell CK, Romanek KM, Heh V (2007) Impaired functioning and quality of life in severe migraine: the role of catastrophizing and associated symptoms. Cephalalgia 27(10):1156-1165.

Leeuw M, Goossens ME, Linton SJ, Crombez G, Boersma K, Vlaeyen JW (2007) The

fear-avoidance model of musculoskeletal pain: current state of scientific evidence. J Behav Med 30(1):77-94.

Martin PR (2010) Managing headache triggers: think,coping' not, avoidance'. Cephalalgia 30(5):634-637.

Vlaeyen JW, Linton SJ (2000) Fear-avoidance and its consequences in chronic musculoskeletal pain: a state of the art. Pain 85(3):317-332.

\section{WS27 - Akupunktur für „Ahnungslose“}

\section{Irnich}

Klinik für Anaesthesiologie, Interdisziplinäre Schmerzambulanz, München, Deutschland

Die Akupunktur besitzt aufgrund nachgewiesener Effektivität [1-6] einen festen Stellenwert in der Behandlung chronischer Schmerzen. Ca. 30.000 Ärzte wenden Akupunktur zumindest gelegentlich an. Die gesetzliche Krankenversicherung übernimmt bei zwei Diagnosen die Kosten.
Im ersten Teil gibt der Workshop einen Überblick über Geschichte und traditionelle Systeme der Akupunktur und stellt die grundlegenden Denkund Therapieansätze inklusive ihrer modernen Erweiterungen (Triggerpunkt-Akupunktur, Mikrosystem-Akupunktur) dar. Im Hauptteil werden dann pragmatische, symptombezogene Therapiekonzepte bei verschiedenen Schmerzerkrankungen vorgestellt, demonstriert und geübt. Vorkenntnisse sind nicht notwendig, aber auch nicht hinderlich, denn aktuelle Entwicklungen werden integriert.

\section{Literatur}

1. Vickers AJ, Cronin AM, Maschino AC, Lewith G, MacPherson H, Foster NE et al (2012) Acupuncture for chronic pain: individual patient data meta-analysis. Archives of internal medicine 22;172(19):1444-1453.

2. Trinh K, Graham N, Irnich D, Cameron ID, Forget M (2016) Acupuncture for neck disorders. Cochrane Database Syst Rev (5):CD004870. doi: 10.1002/14651858. CD004870

3. Linde $K$, Allais G, Brinkhaus B, Fei Y, Mehring M, Vertosick EA, Vickers A, White AR (2016) Acupuncture for the prevention of episodic migraine. Cochrane Database Syst Rev (6):CD001218. doi: 10.1002/14651858.CD001218

4. Linde K, Allais G, Brinkhaus B, Fei Y, Mehring M, Shin BC, Vickers A, White AR (2016) Acupuncture for the prevention of tension-type headache. Cochrane Database Syst Rev 4:CD007587. doi: 10.1002/14651858.CD007587

5. Vickers AJ, Linde K (2014) Acupuncture for chronic pain. JAMA 311(9):955-956

6. Berman BM, Langevin HM, Witt CM, Dubner R (2010) Acupuncture for chronic low back pain. N Engl J Med 363(5)

\section{WS28 - Interprofessionelle Begutachtung schmerzbedingter Funktions- und Leistungseinschränkungen}

R. Dohrenbusch', T. Grömer

${ }^{1}$ Institut für Psychologie, Abteilung für Methodenlehre, Diagnostik und Evaluation, Bonn, Deutschland, ${ }^{2}$ Universität Erlangen-Nürnberg, Facharzt für Psychiatrie und Psychotherapie, Bamberg, Deutschland

Chronische Schmerzen sind neben psychischen Störungen ein häufiger Grund für eine geminderte Funktions-, Arbeits-, Erwerbs- und Berufsfähigkeit. Psychischen Störungen vergleichbar gelten chronische Schmerzen als multipel determiniert, d.h. sowohl die Erscheinungsform chronischer Schmerzen, als auch deren Verlauf und noch stärker deren Auswirkungen auf das Funktions- und Leistungsniveau der Betroffenen werden als abhängig von vielfältigen körperlichen, psychischen, sozialen und sonstigen personalen und kontextuellen Bedingungen angesehen.

Aufgabe medizinischer und psychologischer Gutachter im Sozial- und Zivilrecht ist es, in einem gegebenen Rechtskontext Fragen entweder zur Kausalität oder zu Art und Ausmaß schmerzbedingter Funktions- und Leistungsbeeinträchtigungen zu beantworten. Die Tätigkeit erfordert Kenntnisse der relevanten rechtlichen Bedingungen und Bewertungsgrundlagen sowie medizinische Kenntnisse zu Art und Ursachen schmerzverursachender Erkrankungen und Störungen. Die Kenntnis psychologischer Theorien und Methoden zwecks Unterscheidung gestörter und nicht gestörter körperlicher und psychosozialer Prozesse sowie zur Beschwerdenvalidierung sind ebenfalls erforderlich. Vor diesem Hintergrund steht die Begutachtung schmerzbedingter Krankheitsfolgen für eine interprofessionelle Zusammenarbeit von Juristen, Ärzten und Psychologen. Ausgangspunkt der gutachterlichen Bewertung schmerzbedingter Funktionseinschränkungen ist die medizinische bzw. körperliche Untersuchung in Verbindung mit Labordiagnostik, körperlicher Funktionsdiagnostik und bildgebender Diagnostik. Teilweise unabhängig von der Art der Störung oder Erkrankung und auch unabhängig von der Schmerzätiologie können die Auswirkungen von Schmerzen auf das Funktions- und Leistungsniveau variieren. Eine gesonderte Analyse und Bewertung des allgemeinen und berufsbezogenen Funktions- und Leistungsniveaus ist daher bei Fragen zu schmerzbedingter Arbeitsunfähigkeit oder fraglicher Berufs- oder Erwerbsminderung in aller Regel geboten. Das Untersuchungsprocedere sieht zumeist einen kontrollierten und aufeinander abgestimmten Einsatz verschiedener Untersuchungszugänge vor, darunter körperliche Untersuchung, Labordiagnostik, Diagnostik mit bildgebenden Verfahren, Exploration, Analyse von Fremdberichten, Verhaltensbeobachtung, normierte Fragebogen, körperliche und kognitive Funktions- und 
Leistungstests sowie Validierungsverfahren/Beschwerdenvalidierungstests. Dabei erlauben psychodiagnostische Testverfahren eine zufallskontrollierte wahrscheinlichkeitsbasierte Schätzung der Merkmalsausprägung mittels wiederholter Messungen. Generell sollten der Interpretation von Beobachtungen, Angaben und Testergebnissen in der Einzelfalldiagnostik geeignete Maßnahmen zur Validierung der individuellen Beobachtungen und Messwerte vorausgehen. Ohne die Kontrolle der motivationalen Voraussetzungen, unter denen klinische oder andere psychologische Befunde erhoben wurden, bleibt die Gültigkeit individueller Angaben und Testergebnisse unbestimmt.

Unabhängig von der zugrundeliegenden Erkrankung bzw. Diagnose sprechen die folgenden gutachterlichen Fragestellungen für den expliziten Einbezug psychologischer bzw. psychodiagnostischer Expertise in die gutachtliche Bewertung schmerzbedingter Funktionsbeeinträchtigungen: Wie gültig (valide) sind individuelle Selbstberichte zu Schmerzen und komorbiden psychischen Störungen?

Wie valide sind die Angaben und Testergebnisse zu kognitiven und emotional-motivationalen Funktions- und Leistungsbeeinträchtigungen

Wie wirken sich einerseits krankheitswertige und andererseits ungestörte, normale (körperliche und psychische) Prozesse auf die Schmerz- und Krankheitsverarbeitung des Probanden aus?

Welche Bedeutung kommt motivationalen Komponenten des Erlebens, Verhaltens und Funktionsniveaus zu, die weitgehend unabhängig von Schmerzen oder bestehenden Erkrankungen/psychischen Störungen sind? Welchen Vorhersagewert haben die in der Begutachtungssituation erhobenen Informationen und Kennwerte für das Verhalten der Betroffenen auBerhalb der Untersuchungssituation in ihrem Alltag und am Arbeitsplatz?

\section{Leitlinien zur Begutachtung schmerzbedingter Funktionseinschränkungen}

Aktuell orientiert sich die Begutachtung von Personen mit chronischen Schmerzen in Deutschland an mehreren konsensusbasierten Leitlinien. Dies sind die Ärztliche Leitlinie für die Begutachtung von Menschen mit chronischen Schmerzen der Deutschen Gesellschaft für Neurologie (DGN) und der Deutschen Gesellschaft für Neurowissenschaftliche Begutachtung (DGNB), die Leitlinie zur Begutachtung von idiopathischen und symptomatischen Kopfschmerzen der Deutschen Migräne- und Kopfschmerzgesellschaft (DMKG) und die Leitlinie zur Begutachtung bei psychischen und psychosomatischen Erkrankungen der Deutschen Gesellschaft für Psychosomatische Medizin und Ärztliche Psychotherapie (DGPM) und dem Deutschen Kollegium für Psychosomatische Medizin (DKPM). Dabei handelt es sich um Orientierungshilfen, die aber nicht rechtsverbindlich sind, d.h. jeder Gutachter kann begründet von ihnen abweichen.

Ein Vergleich der Leitlinien zeigt, dass sie sich nicht nur auf unterschiedliche Störungs- bzw. Krankheitsbilder beziehen, sondern auch innerhalb des gutachterlichen Vorgehens und der Gewichtung von Krankheitsfolgemerkmalen unterschiedliche Akzente setzen. So macht die Leitlinie der DGN, anders als die zur Begutachtung psychischer und psychosomatischer Erkrankungen, eine ätiologisch orientierte Klassifikation chronischer Schmerzen zum Ausgangspunkt der Bewertung schmerzbedingter Leistungseinschränkungen. Diese Empfehlung spiegelt eine verstärkte Ausrichtung neurologischer Begutachtung an möglichen Krankheitsursachen und somit auch an Begutachtungsfragen aus der gesetzlichen Unfallversicherung und dem sozialen Entschädigungsrecht wider. Differenzialdiagnostische bzw. klassifikatorische Unterscheidungen unterschiedlicher Krankheitsbilder bilden auch Schwerpunkte der Leitlinie zur Begutachtung von Kopfschmerzen. Für die Begutachtung chronisch schmerzbedingter Funktionseinschränkungen, wie sie im Schwerbehindertenrecht, in der Rentenversicherung und in der privaten Berufsunfähigkeitsversicherung häufig sind, liegt der Schwerpunkt deutlicher auf der gesonderten Bewertung von Funktions- und Leistungsmerkmalen auch unabhängig von differentialdiagnostischen und nosologischen Zuschreibungen. Hier gibt die Leitlinie der DGPM und DKPM zur Begutachtung psychischer und psychosomatischer Erkrankungen den differenziertesten Bezugsrahmen.
Übereinstimmend legen alle Leitlinien Wert darauf, dass Symptomatik (zeitliche Merkmale, Qualität, Intensität, Auslösebedingungen, Krankheitsverhalten und dessen biopsychosoziale Bedingungen, Art und Wirksamkeit bisheriger Therapie) und Funktionsniveau (Funktions- und Leistungseinschränkungen, Bewältigungsressourcen) umfassend exploriert werden. In der Leitlinie der DGN werden insgesamt über 50 teilweise komplexe Themen genannt, die es zu explorieren gelte, darunter die Symptomatik inklusive Begleitkomplikationen (Komorbidität), deren mutmaßliche Ätiologie, Therapierbarkeit, Funktionalität und Kompensierbarkeit in einem biographischen, sozialen und leistungsbezogenen/beruflichen Kontext.

Zur Funktionsbeschreibung wird in allen Leitlinien auf die Systematik der internationalen Klassifikation der Funktionsfähigkeit, Behinderung und Gesundheit der WHO (ICF) oder deren Abkömmling (Mini-ICF; [3]) in der Leitlinie der DGPM/DKPM) verwiesen. In der Untersuchungspraxis von Personen mit chronischen Schmerzen werden meist zwei sich ergänzende Bewertungslinien verfolgt: Zum einen die Bewertung körperlicher Funktionseinschränkungen von der speziellen Interaktion zwischen Schmerzlokalisation, lokaler Intensität und schmerzbedingter (lokaler) Kraft- und Bewegungseinschränkung, zum anderen unabhängig von dieser Wechselwirkung die dauerhaften Auswirkungen chronischer oder chronisch-rezidivierender Schmerzen auf Parameter der allgemeinen kognitiven und körperlichen Leistungsfähigkeit sowie auf Merkmale der emotionalen Stabilität und psychosozialen Belastbarkeit. Die zuletzt genannte Analyseebene löst sich von einer an der Schmerzlokalisation orientierten Funktionsbewertung.

Nur orientierende Hinweise liefern die schmerzbezogenen Leitlinien dazu, wie Gutachter mit der Flut erhobener Informationen praktisch umgehen sollen. Dabei birgt eine vorwiegend explorative Erhebung einer so großen Zahl von Detailinformationen zu Beschwerden, Persönlichkeitsmerkmalen, Kontextbedingungen, biographischen Einflüssen, Krankheits- und Bewältigungsverhalten, motivationalen Bedingungen, Funktionalitäten, sozialen Bedingungen, Leistungsanforderungen usw. in Verbindung mit freier Beobachtung das Risiko einer uneinheitlichen Verarbeitung und interindividuell unterschiedlichen gutachterlichen Gewichtung der erhobenen Informationen. Diese Probleme können aber durch den Einbezug psychologischer Theorien und Modelle v. a. zu Schmerz-/Krankheitsbewältigung und Funktionsbeschreibung in die Begutachtung und durch die Verwendung standardisierter psychodiagnostischer Verfahren entschärft werden.

Gleiches gilt für Maßnahmen zur Beschwerdenvalidierung. Validierungsfragen behandeln die Leitlinien der DGN und der DGPM/DKPM am ausführlichsten, die DGN-Leitlinie unter der Bezeichnung „Konsistenzprüfung“, die DGPM/DKPM-Leitlinie unter „Tendenziösen Haltungen“. Beide Leilinien listen v. a. eine Vielzahl von Vergleichen zur Konsistenzprüfung auf, nehmen aber keine Gewichtung verschiedener Validierungsmethoden vor. Konsens besteht insofern, als die Übereinstimmung in Bezug auf denselben Sachverhalt (die gleiche Aussage oder Beobachtung, das Testergebnis zur gleichen Fähigkeit) als Maß für die Gültigkeit des Sachverhalts interpretiert wird. Die praktische Umsetzung der Empfehlungen ist aber auch hier dadurch eingeschränkt, dass die Vielzahl der geforderten Vergleiche ohne psychologische Theorien und Methoden dem Sachverständigen nur eingeschränkt praktische Handlungsempfehlungen hinterlässt. Zugleich werden Beschwerdenvalidierungstests zumindest in der Leitlinie zur Schmerzbegutachtung eher zurückhaltend bzw. kritisch bewertet. Psychologische Validierungstests und Kontrollskalen zum Antwortverhalten prüfen die Gültigkeit psychischer Eigenschaften (darunter auch Schmerzen und Auswirkungen von Schmerzen auf das kognitive Funktionsniveau) auf der Grundlage kalkulierter Wahrscheinlichkeiten und wiederholter Messungen zufallskritisch. Insofern gelten die formulierten Vorbehalte hier ausgerechnet denjenigen Verfahren, die unter Berücksichtigung testpsychologischer (wahrscheinlichkeitsbasierter) Prinzipien zur Kontrolle motivationaler Voraussetzungen entwickelt wurden. Für eine pauschal kritische Bewertung psychologischer Testverfahren bei psychodiagnostischen Fragestellungen gibt es keinen evidenzbasierten Hintergrund. In einzelnen Fällen kann eine schwerwiegende Psychopathologie 
wie eine starke Konzentrationsstörung oder deutliche Verlangsamung die Ergebnisse beeinträchtigen, dies bleibt davon unberührt.

Ein weiterer Schwerpunkt der Begutachtung von Personen mit chronischen Schmerzen liegt auf der Analyse der Art, Qualität und Wirksamkeit von Maßnahmen zur Bewältigung und Therapie von Schmerzen und Beeinträchtigungen. Hier zeigen die schmerzbezogenen Leitlinien der DGN und der DMKG, noch stärker aber die Leitlinie zur Begutachtung psychischer und psychosomatischer Erkrankungen der DGPM/DKPM, eine explizite Ausrichtung der Bewertung auch an bewusstseinsnahen und der willentlichen Kontrolle unterliegenden Einflüssen. Die Bereitschaft, auch willentliche (nicht krankhafte oder krankheitswertige) psychische Prozesse in die gutachtliche Bewertung schmerz- und psychisch bedingter Krankheitsfolgen einzubeziehen, hat in den letzten Jahren zugenommen. Sie spiegelt auch die wissenschaftliche Daten- und Kenntnislage wider. Daher sollten willentliche (normalpsychische, verhaltens- und kontextbezogene) Einflüsse auch in angemessener Gewichtung neben Krankheitsoder Störungsmerkmalen in die Prognose der zu erwartenden Funktionsentwicklung einbezogen werden. Außerdem kommt der Analyse des Bewältigungsverhaltens und der Nutzung analgetischer Medikation Bedeutung für die prognostische Bewertung zu. Damit verbunden sind Fragen der Medikamentencompliance, die z. B. über die Bestimmung von $\mathrm{Me}-$ dikamentenspiegeln objektivierbar ist.

Ergänzende Hinweise und Analysen zur gutachtlichen Bewertung von Schmerzbewältigungsprozessen sowie schmerzbedingter Funktions- und Leistungsbeeinträchtigungen liefern die zu den Leitlinien herausgegebenen Kommentare (vgl. [5, 2, 1, 4]).

Schlussfolgerungen: Der Vergleich der Leitlinien macht deutlich, dass die rechtliche Bewertung chronisch schmerzbedingter Krankheitsfolgen gleichermaßen juristische, medizinische und psychologische Kenntnisse und Kompetenzen erfordert. Der medizinische Schwerpunkt liegt auf der Analyse krankheitsbezogener Merkmale und Alltagsbeeinträchtigungen sowie auf ätiologischen und klinisch-funktionellen Aspekten. Der Bedarf an psychodiagnostischer Expertise ergibt sich u. a. aus der Vielzahl zu berücksichtigender biopsychosozialer (krankheitswertiger und nicht krankheitswertiger) Faktoren, deren Erhebung an Selbstauskünfte der Betroffenen sowie an Beobachtungen und nicht an „objektive Befunde“ gebunden sind. Juristische Kenntnisse sind erforderlich, um einschätzen zu können, in welchen rechtlichen Bewertungskontext die Ergebnisse der Begutachtung letztlich gestellt werden. Die Begutachtung von Personen mit chronischen Schmerzen ist daher ein interprofessionelles Arbeitsgebiet, in dem neben der reinen Bewertung des medizinischen Sachverhaltes die psychologisch/psychiatrische Beschreibung und Bewertung der Auswirkungen von Schmerzen auf das Funktions- und Leistungsniveau der Betroffenen sowie die Validierung der Befunde wesentliche Elemente darstellen.

\section{Literatur}

1. Dohrenbusch R (2014). Ärztliche Begutachtung von Menschen mit chronischen Schmerzen - Positionen und Kommentare. JC Schmerzmedizin 3:218-222

2. Evers S, May A, Heuft G, Husstedt IW, Keidel M, Malzacher V, Straube A, Widder B (2010) Die Begutachtung von idiopathischen und symptomatischen Kopfschmerzen. Nervenheilkunde 4:229-241

3. Linden M, Baron S, Muschalla B (2009) Das Mini-ICRF-Rating für psychische Störungen (Mini-ICF-APP). Hogrefe \& Huber, Bern

4. Schneider W, Dohrenbusch R, Freyberger HJ, Henningsen $P$, Irle H, Köllner V, Widder $B$ (2016) Begutachtung bei psychischen und psychosomatischen Erkrankungen. Autorisierte Leitlinien und Kommentare. Hogrefe, Göttingen

5. Widder B, Dertwinkel R, Egle UT, Foerster K, Schiltenwolf M (2007) Leitlinie für die Begutachtung von Schmerzen. Psychotherapeut 52:334-346

\section{WS29 - Fahrtauglichkeit/Arbeitsfähigkeit unter Medikamenten - Aktueller Stand}

\section{Pappenhoff' C. Sauer ${ }^{2}$}

'BG Klinikum Duisburg, Klinik für Schmerzmedizin, Duisburg, Deutschland, ${ }^{2} \mathrm{BG}$ Klinikum Hamburg, Anästhesie, Intensiv-, Rettung- und Schmerzmedizin, Lübeck, Deutschland

Zur Einschätzung von Fahrtauglichkeit und evtl. auch der Arbeitsfähigkeit unter Medikamenten existieren weder Leitlinien noch aktuelle konkrete
Handlungsanweisungen zu einem abgestuften Vorgehen. Aktuelle Empfehlungen, z. B. auch in LONTS oder der Leitlineie zur Fahrbegutachtung beziehen sich immer auf Einzelwirkstoffe. In der Realität werden Schmerzpatienten jedoch verschiedene Wirkstoffgruppen verordnet, wobei jede für sich schon eine Einschränkung bedeuten kann

Der Arbeitskreis Beruf und Schmerz der Deutschen Schmerzgesellschaft beschäftigt sich intensiv mit der Fragestellung, wie in diesen komplexen Situationen verfahren werden kann und was dies für die Arbeitsfähigkeit bedeuten kann. Dabei ist Fahrtauglichkeit nicht gleichzusetzen mit Arbeitsfähigkeit. Um diese Unterschiede herauszuarbeiten, setzt sich der Arbeitskreis Beruf und Schmerz aus Arbeits- und Schmerzmedizinern zusammen.

\section{WS30 - Chronische Schmerzen bewältigen oder akzeptieren?}

\section{B. Kappis', M. von Wachter ${ }^{2}$}

'Universitätsmedizin der Johannes Gutenberg-Universität, Klinik für Anästhesiologie/Schmerzambulanz, Mainz, Deutschland, ${ }^{2}$ Ostalb-Klinikum Aalen, Klinik für Psychosomatik, Aalen, Deutschland

Da sich in der Versorgung chronischer Schmerzpatienten einseitig somatisch orientierte Behandlungsansätze als unzureichend erwiesen haben, sind bei der Therapie entsprechend den bio-psycho-sozialen Krankheitsanteilen interdisziplinäre Behandlungsansätze gefragt. Neben der medikamentösen Schmerztherapie und körperlicher Aktivierung spielt dabei die Psychotherapie eine entscheidende Rolle.

Zwei Ansätze erscheinen dabei widersprüchlich: Einerseits gegen die Schmerzen ankämpfen, sie kontrollieren, ignorieren oder bewältigen und andererseits auf sie zugehen, die Schmerzen akzeptieren. Wann macht was Sinn? Die beiden Ansätze werden dargestellt, entsprechende Erklärungsmodelle für Patienten gezeigt und strategische Unterschiede im therapeutischen Vorgehen unter Berücksichtigung verschiedener Störungsbilder wie chronisches Schmerzsyndrom nach Gewebsschädigung, funktionelle Schmerzerkrankungen und Fibromyalgiesyndrom werden herausgearbeitet.

Der Schwerpunkt dieses Workshops liegt auf der Demonstration beispielhafter Interventionen. Aktive Teilnahme erwünscht!

\section{WS31 - Studien lesen und wirklich verstehen}

\section{T. Dresler', K. Lüdtke ${ }^{2}$}

${ }^{1}$ Klinik für Psychiatrie und Psychotherapie, Psychophysiologie und Optische Bildgebung, Tübingen, Deutschland, ${ }^{2}$ Universitätsklinikum Hamburg Eppendorf, Institut für Systemische Neurowissenschaften, Hamburg, Deutschland

Die Publikation von Forschungsergebnissen in internationalen Fachzeitschriften und auf Kongressen zählt zu den wichtigsten Aufgaben von Wissenschaftlern. Leser wissenschaftlicher Studien, insbesondere Leser mit einem vorwiegend klinischen Hintergrund, „überspringen“ dabei oft den Methodikteil. Die Ergebnisse und Schlussfolgerungen einer Studie können jedoch abhängig von den gewählten Methoden sehr unterschiedlich ausfallen. Für die Beurteilung der Glaubwürdigkeit und Güte von Studienergebnissen und der daraus gezogenen Schlussfolgerungen sind gute methodische Kenntnisse demnach entscheidend. Auch ein Basiswissen für die Anwendung der häufigsten statistischen Methoden trägt zu der sicheren Einschätzung der Zuverlässigkeit von Studienergebnissen bei.

In diesem fallbasierten Workshop werden häufig auftretende methodische und statistische Mängel (z. B. Design, Stichprobengröße, Datenauswertung, Ergebnisdarstellung) beleuchtet. Praktische Werkzeuge zur Beurteilung der methodischen Qualität von Studien und Übersichtsarbeiten werden vorgestellt. Anhand von kurzen Beispielen können Teilnehmer ihre eigenen Kenntnisse überprüfen und im Plenum diskutieren. Dieser Kurs richtet sich insbesondere an Kliniker, Studierende und Doktoranden. 
WS32 - Sinnvolle Diagnostik und Therapie bei neuropathischen Schmerzen - ein Fallseminar

\section{J. Hellriegel, S. Rehm}

Klinik für Neurologie, Universitätsklinikum Schleswig-Holstein, Sektion Neurologische Schmerzforschung und -therapie, Kiel, Deutschland

Neuropathische Schmerzen unterscheiden sich ätiologisch und symptomatisch von chronischen Schmerzen, bei denen das Nervensystem intakt ist. Auch die Therapie neuropathischer Schmerzen unterscheidet sich deutlich von der Therapie nozizeptiver Schmerzen - dies macht eine klinische Differenzierung zwischen diesen beiden Schmerzformen so wichtig! Anhand von Beispielen soll in einem interaktiven Fallseminar die klinische Manifestation neuropathischer Schmerzsyndrome vorgestellt und die über die körperliche Untersuchung und Anamnese hinausgehenden apparativen Untersuchungstechniken erläutert werden. Neurophysiologische Untersuchungsverfahren ermöglichen eine Funktionsdiagnostik des peripheren und zentralen Nervensystems und bildgebende Verfahren können Läsionen im schmerzverarbeitenden System aufzeigen. Deren Chancen aber auch Fallstricke sollen ebenso diskutiert werden wie eine mögliche durch intensive apparative Diagnostik entstehende iatrogene Chronifizierung oder eine Verunsicherung von Patienten durch vermeintlich auffällige aber irrelevante Befunde. Als weiteren Schwerpunkt werden in diesem Seminar die aktuellen Therapiealgorithmen für neuropathische Schmerzen vorgestellt und Therapiekonzepte für beispielhafte Patienten entwickelt. Dabei soll insbesondere auch auf die Möglichkeiten der Kombinationstherapie und auf mögliche Fehlerquellen bei der Therapieplanung dieser häufig hoch chronifizierten Patienten eingegangen werden. Durch die Möglichkeit der Workshopteilnehmer eigene Fallbeispiele im Vorfeld an die Referenten zu übermitteln, soll eine hohe Praxisrelevanz für die Teilnehmer erreicht werden.

\section{WS33 - Biofeedback-Therapie bei chronischen Schmerzen}

\section{A. Diezemann \\ 'DRK-Schmerz-Zentrum Mainz, Tagesklinik für interdisziplinäre \\ Schmerztherapie, Mainz, Deutschland}

Biofeedback stellt in der Schmerztherapie ein etabliertes Verfahren dar. Durch die systematische Rückmeldung messbarer körperlicher Signale können Patienten mit chronischen Schmerzen unterschiedlich profitieren: Zusammenhänge zwischen seelischen mit körperlichen Prozessen können dargestellt werden, was dem Patienten das Verständnis für ein bio-psychosoziales Modell erleichtert. Darüber hinaus kann der Patient lernen, spezifische physiologische Veränderungen hervorzurufen. Dies fördert die Entwicklung einer Selbstwirksamkeitserwartung, welche einen wesentlichen Aspekt einer günstigen Schmerzbewältigung darstellt.

Das Seminar bietet einen Überblick über die Biofeedbackanwendung in der Schmerztherapie, die vermuteten Wirkmechanismen und die Wirksamkeit bei verschiedenen Schmerzbildern. Die unterschiedlichen Methoden werden mit einem Mehrkanalgerät und tragbaren Ein-Kanalgeräten demonstriert. Hierbei werden Aspekte der Diagnostik, Modellvermittlung, der Einsatz von Biofeedback bei Rücken- und Nackenschmerzen, Kopfschmerz und allgemeine Entspannungstechniken mit Hilfe von Biofeedback vorgestellt.

\section{Literatur}

1. Martin A, RiefW W (Hrsg) (2008).Wie wirksam ist Biofeedback? Hans Huber, Bern

2. Rief, W, Birbaumer, N (Hrsg) (2010) Biofeedback: Grundlagen, Indikationen, Kommunikation, Vorgehen. Schattauer, Stuttgart

\section{P01 - Akutschmerz/Schmerz nach Operationen}

\author{
P01.01 - Schmerzen in der Notfallaufnahme eines Krankenhauses \\ - Inzidenz, Intensität und Lokalisation \\ P. Ristau', S. Pfeuffer ${ }^{2}$ \\ 'Evangelische Hochschule Darmstadt, Fachbereich Pflege- \\ und Gesundheitswissenschaften, Weiterstadt, Deutschland, \\ ${ }^{2}$ Evangelische Hochschule Darmstadt, Fachbereich Pflege- und \\ Gesundheitswissenschaften, Darmstadt, Deutschland
}

Einleitung, Ausgangslage: Schmerz in der Notaufnahme ist ein weit verbreitetes, aber interdisziplinär wenig erforschtes Phänomen.

Fragestellungen: In der Zentralen Notaufnahme (ZNA) eines Krankenhauses der Grund- und Regelversorgung in Hessen sollen zunächst die folgenden Fragestellungen untersucht werden: Wie hoch ist der Anteil derer Patienten, die während einer Behandlung in der Zentralen Notfallaufnahme über Schmerzen berichten? Handelt es sich dabei um akute oder chronische Schmerzen? Gibt es Unterschiede zwischen den behandelnden Fachabteilungen bezüglich der Inzidenz, Intensität und Lokalisation der Schmerzen?

Methodik, Population und Stichprobe: Bei dieser Untersuchung handelt es sich um eine papiergebundene, quantitative Befragung. Die Population bilden die in der ZNA behandelten Patienten, die Stichprobe umfasst die im 14tägigen Untersuchungszeitraum dort behandelten Patienten. Ein Informed Consent ist Voraussetzung zur Befragung.

Ethische Überlegungen: In die Untersuchung werden lediglich Patienten der somatischen Fachabteilungen einbezogen. Ausgeschlossen sind Patienten, die auf die Palliativ- oder Intensivstation aufgenommen wurden, dement oder desorientiert oder nicht der deutschen Sprache mächtig sind. Datenerhebung: Das Austeilen der Fragebögen erfolgt an ambulante Patienten unmittelbar nach Abschluss deren Behandlung noch vor Ausgabe des Entlassungsbriefes, bei stationären am Tag nach der Aufnahme. Dazu werden die Patienten persönlich aufgesucht. Durch externe Forscher wird soziale Erwünschtheit reduziert.

Ergebnisse: Insgesamt konnten im Untersuchungszeitraum 155 Fragebögen ausgegeben werden (Rücklauf 64,52\%, $n=100 ; 44 \%$ männlich; $45 \%$ weiblich; mittleres Alter 54,1 Jahre). $69 \%$ der Patienten gaben Schmerzen an, davon wiederum gaben $79,7 \%$ akute und $15,9 \%$ chronische Schmerzen an. 85,5\% nannten Schmerzen als Hauptgrund für ihre Vorstellung. Der mittlere Schmerzwert vor einer analgetischen Behandlung betrug über alle Abteilungen 5,9 $(n=50)$ auf einer zehnstufigen Numerischen Analogskala. (Innere Medizin 5,8, $n=28$; Allgemeinchirurgie 7,0, $n=4$; Unfallchirurgie $6,2, n=12)$. In der Inneren Medizin klagte ein Großteil über Schmerzen im Abdomen (45,7\%, $n=16)$, in mehreren Körperbereichen $(17,1 \%, n=6)$ oder in der Brust $(14,3 \%, n=5)$. In der Allgemeinchirurgie klagten alle Patienten über abdominelle Schmerzen $(100 \%, n=4)$, in der Unfallchirurgie überwiegend über Schmerzen in den Extremitäten $(45,5 \%, n=5)$ sowie in der Brust $(18,2 \%, n=2)$.

Schlussfolgerungen und Empfehlungen: Schmerzen stellen eine Hauptursache für Patienten dar, die ZNA eines Krankenhauses aufzusuchen. Oftmals sind diese interventionspflichtig. Die Lokalität unterscheidet sich zwischen den Fachabteilungen. Besonders in der ZNA bedarf es daher eines interdisziplinären Schmerzkonzeptes.

\section{P01.02 - Regionalanästhesie der oberen Extremität bei Handphlegmone}

M. Dillinger ${ }^{1}$, M. Huber ${ }^{2}$, N. Lindenberg ${ }^{3}$, C. Wiese ${ }^{4}$, C. Lassen ${ }^{3}$ ${ }^{1}$ Klinikum Garmisch-Partenkirchen, Anästhesie und Operative Intensivmedizin, Garmisch-Partenkirchen, Deutschland, ${ }^{2}$ Universitätsklinikum Regensburg, Klinik und Poliklinik für Unfallchirurgie, Regensburg, Deutschland, ${ }^{3}$ Universitätsklinikum Regensburg, Klinik für Anästhesiologie, Regensburg, Deutschland, ${ }^{4}$ Herzogin Elisabeth Hospital Braunschweig, Klinik für Anästhesiologie und Intensivmedizin, Braunschweig, Deutschland 
Hintergrund: Die kontinuierliche Regionalanästhesie (RA) der oberen Extremität ist bei Operationen an Hand/Unterarm ein etabliertes Narkoseverfahren. Während eine Infektion im Zugangsbereich der Nervenblockade eine anerkannte Kontraindikation darstellt, ist unklar inwieweit eine zugangsferne Infektion zu berücksichtigen ist. In dieser retrospektiven Untersuchung sollte an einem Kollektiv von Patienten mit Handphlegmone der Einfluss der kontinuierlichen RA auf das weitere Outcome im Vergleich zu einer Allgemeinanästhesie beurteilt werden. Ebenso sollte untersucht werden, ob bei einer kontinuierlichen RA bei zugangsferner Infektion ein erhöhtes Infektionsrisiko des Katheters im Vergleich zu einem gematchten Kontrollkollektiv bei aseptischer Operation besteht.

Methodik: Es wurden alle Patienten untersucht, die an unserer Klinik im Zeitraum vom 1.1.2007 bis 31.5.2013 wegen einer Handphlegmone operiert wurden. Hierfür wurden demographische, prozedurale und postoperative Informationen erhoben. Zum Vergleich des Infektionsrisikos zwischen Patienten mit Handphlegmone und aseptischer Operation wurde aus einem Kollektiv von Patienten mit elektiver Operation an der Hand und kontinuierlicher RA eine Kontrollgruppe gebildet, die nach Alter, Geschlecht und ASA-Kategorie gematcht wurde.

Ergebnisse: Von insgesamt 141 Patienten, die wegen einer Handphlegmone operiert wurden bekamen 54 Patienten eine kontinuierliche Regionalanästhesie (KRA), 57 eine Allgemeinanästhesie (AA) und 30 eine singleshot Regionalanästhesie. Die Gruppen KRA und AA unterschieden sich nicht signifikant in den demographischen Parametern und prozeduralen Parametern. Als Zeichen der Infektionsschwere war der CRP Wert in der Gruppe AA gegenüber der Gruppe KRA signifikant erhöht (69,2 mg/l vs. $35,2 \mathrm{mg} / \mathrm{l}, p=0,008)$. Postoperativ zeigte sich in der Gruppe AA eine verlängerte Dauer der Antibiotikagabe (11,1 Tage vs. 7,4 Tage, $p=0,04)$ und einen Trend zur längeren Krankenhausverweildauer (16,8 Tage vs. 12,0, $p=0,069)$. Im Vergleich der Gruppe KRA zur Kontrollgruppe mit Regionalanästhesie bei aseptischer Operation zeigte sich bei gleicher Liegedauer des Katheters (4,0 Tage) kein erhöhtes Risiko für eine Infektion an der Einstichstelle des Katheters.

Diskussion: In unserem untersuchten Patientenkollektiv zeigt sich kein erhöhtes Risiko für Patienten mit kontinuierlicher Regionalanästhesie bei einer Handphlegmone. Im Gegenteil zeigt sich eine kürzere Notwendigkeit der Antibiotikagabe und ein deutlicher Trend zur verkürzten Krankenhausverweildauer. Ob dies ursächlich auf die Regionalanästhesie zurückzuführen ist, kann durch diese retrospektive Untersuchung nicht geklärt werden. Unserer Meinung nach ist die grundsätzliche Wahl eines Regionalanästhesieverfahrens bei einstichferner Infektion gerechtfertigt. Neben der Präferenz des Patienten könnte auch der womöglich positive Einfluss der Regionalanästhesie auf den Verlauf der Infektion in der Entscheidungsfindung berücksichtigt werden.

\section{P01.03 - Validierung eines Risikoscores zur Vorhersage ausgeprägter postoperativer Schmerzen}

C. Vahldieck', M. Lindig' ', C. Biet' ' C.Nau' ${ }^{1}$, M. Hüppe ${ }^{1}$

'Universität zu Lübeck, Klinik für Anästhesiologie und Intensivmedizin, Lübeck, Deutschland

Einleitung und Fragestellung: Präoperative Schmerzerwartung und schon vor einer Operation bestehende Schmerzen, die von Patienten als beeinträchtigend erlebt werden (dysfunktionale vorbestehende Schmerzen), sind unabhängige Risikofaktoren für ausgeprägte postoperative Schmerzen [1]. Unsere Untersuchung soll zeigen, ob ein aus diesen Variablen gebildeter Risikoscore für die Vorhersage ausgeprägter postoperativer Schmerzen geeignet ist.

Methodik:ach positivem Votum der lokalen Ethikkommission nahmen an der Untersuchung 501 Patienten im Alter zwischen 18 bis 94 Jahren teil, die sich einem elektiven Eingriff in chirurgischen Abteilungen des UKSH Campus Lübeck unterzogen.

Präoperativ wurde von den Patienten ein Schmerz-Risikofragebogen ausgefüllt, der aus 5 Items besteht: die ersten drei Items betreffen präoperative Schmerzen, deren Intensität und schmerzbedingte Beeinträchtigungen (Beeinträchtigungen mit NRS $>3$ sind dysfunktionale Schmerzen). Die bei- den letzten Items beziehen sich auf die postoperative Schmerzerwartung und deren Intensität (11-stufige NRS).

Ein hoher Risikoscore war festgelegt als „präoperativ vorbestehende schmerzbedingte Beeinträchtigung mit NRS $>3$ “" (entspricht dysfunktionalem vorbestehenden Schmerz) verbunden mit „Schmerzerwartung NRS $>4$ “ für postoperativen Schmerz.

Am Tag nach der Operation fand eine Bestimmung der mittleren postoperativen Schmerzintensität mit Hilfe einer 11-stufigen Numerischen Rating-Skala (NRS mit $0=$ kein Schmerz und $10=$ stärkster vorstellbarer Schmerz) statt. Als hoher postoperativer Schmerz wurde eine Schmerzstärke von NRS > 4 definiert. Zusätzlich erhobene Variablen waren Art, Dauer und Anästhesieform des Eingriffs, eine Schweregradeinteilung nach ISOS (International Surgical Outcomes), sowie soziodemographische Patientenmerkmale. Die Datenerhebung war in das Klinikprojekt QUIPS eingebunden.

Ergebnisse: Patienten mit hoher Schmerzerwartung berichteten höhere postoperative Schmerzen $(p<0,001)$ und Patienten mit dysfunktionalem vorbestehenden Schmerz höhere als Patienten ohne oder mit funktionalem vorbestehendem Schmerz $(p<0,05)$. Patienten mit hoher Schmerzerwartung verbunden mit dysfunktionalem vorbestehenden Schmerz wiesen am häufigsten ausgeprägte postoperative Schmerzen auf. Die Konfigurationsfrequenzanalyse ergab für diese Kombination in Verbindung mit hohem postoperativem Schmerz einen signifikanten Typen $(p<0,001)$.

Diskussion und Schlussfolgerung: Auf Patienten mit hoher Schmerzerwartung verbunden mit dysfunktionalem präoperativen Schmerz sollte postoperativ besonders geachtet werden. Diese Patienten sind mit dem Schmerz-Risikoscore im klinischen Alltag leicht und ökonomisch zu identifizieren.

\section{Literatur}

1. Clasen K (2015) Geschlechtsunterschiede in psychologischen Risikofaktoren für postoperative Schmerzen. Dissertation, Universität zu Lübeck

\section{P01.04 - Schmerzbewältigungsstrategien und postoperative Outcomes nach Hysterektomie}

J. Scheel', M. Strupf', R. Sitt/2, M. Beckmann ${ }^{3}$, F. Thiel ${ }^{3}$, N. Grießinger ${ }^{2}$, S. Lautenbacher $^{7}$

'Otto-Friedrich-Universität Bamberg, Physiologische Psychologie, Bamberg, Deutschland, ${ }^{2}$ Universitätsklinik Erlangen, Schmerzzentrum, Erlangen, Deutschland, ${ }^{3}$ Universitätsklinik Erlangen, Frauenklinik, Erlangen, Deutschland

Hintergrund: Durch schmerzbezogene Emotionen und Kognitionen (z. B. Schmerzangst, -katastrophisieren und -hypervigilanz) können postoperative Outcomes wie Schmerzintensität und schmerzbezogene Beeinträchtigung vorgesagt werden. In Bezug auf Schmerzbewältigungsstrategien ist die Studienlage hierzu heterogen. Ziel der vorliegenden Studie ist daher die Überprüfung postoperativer Schmerzbewältigungsstrategien hinsichtlich (1) ihrer zeitlichen Stabilität, (2) ihrer Interkorrelationen, (3) ihres Zusammenhangs mit präoperativen schmerzbezogenen Emotionen und Kognitionen und (4) ihres Zusammenhangs mit postoperativen Outcomes. Die Ergebnisse könnten zur Weiterentwicklung psychologischer Prophylaxekonzepte herangezogen werden, deren Effekte bisher positiv, aber eher gering sind.

Methoden: Bei 61 Hysterektomie-Patientinnen (45,9 $\pm 4,5$ Jahre) wurden am Tag vor der Operation Schmerzangst, -katastrophisieren und -hypervigilanz erhoben. Am Tag der Entlassung aus der Klinik wurden Schmerzbewältigungsstrategien (Prädiktoren) mittels des FESV-BW (Geissner, 2001; Subskalen: Handlungsplanungskompetenzen, Kognitive Umstrukturierung, Kompetenzerleben, Mentale Ablenkung, Gegensteuernde Aktivitäten, Ruhe \& Entspannung) erfasst. Postoperative Outcome-Variablen waren Schmerzintensität (numerische Ratingskala), aktivitätsbezogene schmerzbezogene Beeinträchtigung (PDI, Nagel et al., 2002) und psychische schmerzbezogene Beeinträchtigung (FESV-BE, Geissner, 2001; Subskalen: Hilflosigkeit \& Depression, Angst, Ärger), diese wurden am Tag der Entlassung aus der Klinik sowie 6 Wochen, 3 Monate und 6 Monate postoperativ untersucht. 
Ergebnisse: Die verschiedenen postoperativen Schmerzbewältigungsstrategien bleiben über den postoperativen Zeitraum hinweg stabil und sind größtenteils signifikant positiv miteinander korreliert. Zu allen Messzeitpunkten (besonders in der akuten postoperativen Phase) zeigen sich signifikante Zusammenhänge zwischen postoperativen Schmerzbewältigungsstrategien und Outcome-Variablen. Hierbei kann ab 6 Wochen nach der Operation nur noch schmerzbezogene Beeinträchtigung vorhergesagt werden, die Schmerzintensität lediglich im Akutbereich. Außerdem sind Kompetenzerleben, Gegensteuernde Aktivitäten und Kognitive Umstrukturierung von höherer Relevanz als die übrigen Schmerzbewältigungsstrategien. Auch zeigen sich nur für diese drei Variablen Zusammenhänge mit präoperativen schmerzbezogenen Emotionen und Kognitionen.

Diskussion: Assoziationen zwischen postoperativen Schmerzbewältigungsstrategien und postoperativen Outcomes zeigen sich am stärksten in der akuten postoperativen Phase, möglicherweise aufgrund der geringen Auftretenshäufigkeit persistierender postoperativer Schmerzen in unserer Stichprobe. Von höchster Relevanz scheinen die beiden Schmerzbewältigungsstrategien Kompetenzerleben und Gegensteuerende Aktivitäten zu sein. Diese könnten in prä- oder perioperativen psychologischen Prophylaxeprogrammen besonders fokussiert werden, um zu überprüfen ob sie Resilienzfaktoren darstellen.

\section{Literatur}

Geissner E (2001) Fragebogen zur Erfassung der Schmerzverarbeitung FESV. Göttingen: Hogrefe.

Nagel B, Gerbershagen HU, Lindena G et al (2002) Development and evaluation of the multidimensional German pain questionnaire. Schmerz 16:263-270.

\section{P01.05 - Beeinflusst das Geschlecht des Untersuchers die Bewertung akuter postoperativer Schmerzen bei Männern und Frauen? Eine prospektive Studie an Patienten nach Sternotomie.}

C. Meyer-Frießem', P. Szalaty', J. Bettmann', C. Krämer', E. Meier-Gerdhing', P. Zahn' ${ }^{1}$ E. Pogatzki-Zahn ${ }^{2}$

'Berufsgenossenschaftliches Universitätsklinikum Bergmannsheil, Klinik für Anästhesiologie, Intensiv-, Palliativ- und Schmerztherapie, Bochum, Deutschland, ${ }^{2}$ Universitätsklinikum Münster, Klinik für Anästhesiologie, postoperative Intensivmedizin und Schmerztherapie, Münster, Deutschland

Hintergrund: Klinisch wird die Intensität von Schmerzen von jedem Patienten subjektiv und individuell beurteilt. Dabei scheint auch der Untersucher selbst diese Einschätzung zu beeinflussen. Bisher existieren jedoch nur inkonsistente Daten unter experimentellen Bedingungen an gesunden Probanden (Levine et al. 1991, Kallai et al. 2004, Aslaksen et al. 2007). Fragestellung: Primäres Ziel dieser prospektiven Studie war es zu untersuchen, inwiefern sich die Einschätzung der Intensität von akuten postoperativen Schmerzen anhand der numerischen Ratingskala (NRS) nach Thorakotomie bei Männern und Frauen abhängig vom Geschlecht des Untersuchers unterscheidet.

Methodik: Nach Zustimmung der Ethik-Kommission der Ruhr-Universität Bochum und schriftlicher Einverständniserklärung wurden insgesamt 165 Männer und Frauen nach einer herz-thoraxchirurgischen Operation mit Sternotomie zwischen 2012 und 2015 in die Studie eingeschlossen. Es erfolgte die konsekutive Zuteilung von männlichen $(\mathrm{m})$ und weiblichen $(\mathrm{w})$ Patienten zu einem von zwei männlichen $(M)$ oder einem von zwei weiblichen (W) Untersuchern. Alle Patienten waren hinsichtlich der Hypothese der Studie verblindet. Die Befragung zur Schmerzintensität erfolgte am ersten und zweiten postoperativen Tag jeweils morgens und abends auf der Intensivstation anhand der Numerischen Ratingskala $(0=$ kein Schmerz, $10=$ maximal vorstellbarer Schmerz). Die Untersuchungszeitpunkte, das Vorgehen bei der Befragung als auch das postoperative Analgesie-Konzept waren standardisiert. Kruskal-Wallis oder Mann-Whitney-U-Test; $p<0,05$. Ergebnisse: Männer und Frauen werteten über zwei Tage ihre Schmerzen auf der NRS bei 3,49 2,06. Im Durchschnitt nahm die Schmerzintensität zum letzten Untersuchungszeitpunkt ab (H-Test: $p=0,000)$. 12,7\% Patienten nahmen aus verschiedenen Gründen nicht mehr an der letzten Untersuchung teil. Männliche und weibliche Patienten schätzten ihre Schmerzintensität ähnlich ein $(p=0,869, \mathrm{z}=-0,164)$. Am zweiten post- operativen Tag werteten alle Patienten $(\mathrm{m} / \mathrm{w})$ weiblichen Untersuchern $\left(\mathrm{NRS}_{\mathrm{POT} 2}=3,44 \pm 2,50\right)$ gegenüber insgesamt ihre Schmerzen als intensiver als männlichen Untersuchern ( $\left.\mathrm{NRS}_{\mathrm{POT} 2}=2,40 \pm 2,22 ; p=0,000, \mathrm{z}=-3,534\right)$. Zugleich beurteilten männliche Patienten weiblichen Untersucherinnen gegenüber ihre Schmerzintensität signifikant höher als männlichen Untersuchern (NRS 3,88 vs. 2,$72 ; p=0,014, \mathrm{z}=-2,47$ ).

Diskussion: Auch im klinischen Setting scheint das Geschlecht des Untersuchers eine Rolle bei der Schmerzeinschätzung zu spielen. Weiblichen Untersuchern gegenüber werden Schmerzen insgesamt als intensiver angegeben. Vor allem Männer geben weiblichen Untersuchern höhere Schmerzintensitäten als männlichen Untersuchern an. Dieser Aspekt sollte im klinischen Alltag bei der Diagnostik und Therapie von akuten Schmerzen Beachtung finden.

\section{P01.06 - Wie kann die Qualität der postoperativen Analgesie bei Kindern verbessert werden?}

S. Lüscher', I. Wilhelm' ', M. Book' , F. Stüber', U. Stamer ${ }^{2}$

'Inselspital Universität Bern, Klinik für Anästhesiologie und

Schmerztherapie, Bern, Schweiz, ${ }^{2}$ Inselspital Universität Bern, Klinik für Anästhesiologie und Schmerztherapie, Bern, Schweiz

Hintergrund: Postoperative Schmerzen verzögern die Genesung, verursachen Beschwerden und Leiden und erhöhen die Kosten im Gesundheitswesen. Dieses gilt nicht nur für Erwachsene, sondern auch für Kinder und Jugendliche. Das internationale Projekt PAIN Out infant [1] bietet die Möglichkeit, das schmerzrelevante Outcome nach Operationen bei Kindern zu evaluieren und die eigenen Ergebnisse mit denen anderer Klinken zu vergleichen. Dadurch können evtl. vorhandene Defizite aufgedeckt und Veränderungen in der Behandlung umgesetzt werden.

Methodik: Zur Qualitätsverbesserung wurde die Erfassung der klinischen Routinedaten eingeführt (ClinicalTrials.gov.Identifier NCT0203835). Bei Kindern $>4$ Jahre wurde das schriftliche Einverständnis der Eltern, bei Jugendlichen zusätzlich ihre Einwilligung eingeholt. Anhand des standardisierten PAIN-OUT infant Registers wurden Daten zu Demographie, Operation, Anästhesie und perioperativen Schmerztherapie erhoben. Der kindergerechte Fragebogen zum schmerzrelevanten Outcome wurde am ersten postoperativen Tag vom Kind/Jugendlichen, bei jüngeren Kindern zusammen oder allein durch die Eltern, ausgefüllt. Anhand der revidierten Gesichterskala nach Hicks [2] und „Ja-Nein-Fragen“ wurden im Fragebogen Informationen zur schmerzbedingten Beeinträchtigungen und Nebenwirkungen der Schmerztherapie erfragt. Im Benchmarking wurden die eigenen Ergebnisse mit denen anderer teilnehmender Kliniken verglichen. Zusätzlich wurden die perioperativ verabreichten Nichtopioidanalgetika ausgewertet.

Ergebnisse: 3331 Patienten, davon 291 (44\% Mädchen) des Inselspitals, wurden eingeschlossen. Das Benchmarking erlaubte den Vergleich mit 16 anderen Krankenhäusern. Beim Ruheschmerz (NRS median (IQR): $0(0 / 2))$, Schmerz in Bewegung (2 (2/4)) und dem stärksten Schmerz (6 (2/8)) lag die Klinik im vorderen Drittel. Hingegen klagten $74 \%$ der Patienten über Müdigkeit, $52 \%$ über Übelkeit/Erbrechen, das schlechteste Ergebnis im Krankenhausvergleich. Als Konsequenz wurde vor 5 Monaten eine standardisierten PONV Prophylaxe eingeführt. Dadurch konnte die PONV-Rate um die Hälfte gesenkt werden. In $82 \%$ der Fälle erhielten die Kinder intraoperativ Paracetamol allein oder in Kombination mit anderen Schmerzmitteln. Diclofenac wurde bei $29 \%$, Metamizol bei $15 \%$ gegeben. Auf den Stationen wurden vorwiegend Paracetamol (82\%), seltener Metamizol (39\%), Ketorolac (32\%), Ibuprofen (26\%) und Diclofenac $(8 \%)$ verwendet.

Zusammenfassung: Die Auswertung der PAIN OUT Infant Daten vermittelt Informationen aus dem klinischen Alltag. Sie zeigt, in welchen Bereichen Verbesserungen angezeigt sind, z. B. bei der PONV-Prophylaxe. Die bevorzugte Anwendung von Paracetamol sollte überdacht werden.

\section{Literatur}

1. www.pain-out.eu

2. Hicks CL et al (2001) The Faces Pain Scale-Revised: toward a common metric in pediatric pain measurement. Pain 93:173-183 


\section{P02 - Nicht-pharmakologische Therapieverfahren/ Pharmakologische Therapie des Schmerzes}

\section{P02.01 - Einfluss Transkutaner Elektrischer Nervenstimulation (TENS) Auf Parameter Der Quantitativ Sensorischen Testung (QST)}

\author{
L. Schicht ${ }^{1}$, J. Seip ${ }^{1}$, U. Schwanebeck ${ }^{2}$, R. Sabatowski', R. Sabatowski ${ }^{3}$,
} G. Goßrau ${ }^{4}$

'UniversitätsSchmerzCentrum, Universitätsklinikum „Carl Gustav Carus", Dresden, Deutschland, ${ }^{2}$ Koordinierungszentrum für klinische Studien Dresden, Medizinische Fakultät Carl Gustav Carus der Technischen Universität Dresden, Dresden, Deutschland, ${ }^{3}$ Klinik für Anästhesiologie und Intensivtherapie, Universitätsklinik Dresden, Dresden, Deutschland, ${ }^{4}$ Universitätsklinikum Carl Gustav Carus, UniversitätsSchmerzCentrum Schmerzambulanz, Dresden, Deutschland

Einleitung: TENS gilt als supportives Verfahren in der Therapie akuter und chronischer Schmerzen. Wirkmechanismus ist eine Modifikation der neuralen Transmission u. a. durch Freisetzung endogener Opiate und inhibitorischer Neurotransmitter. Bisherige Ergebnisse zur TENS-induzierten Veränderung der sensorischen Wahrnehmung sind kontrovers bei uneinheitlichen Messverfahren und heterogenen Versuchspopulationen. Patienten und Methoden: Mit Hilfe des standardisierten Untersuchungsverfahrens der QST wurde in dieser offenen Studie der qualitative und quantitative Einfluss auf sensorische Parameter Gesunder und chronischer Schmerzpatienten unter transkutaner Hochfrequenzstromapplikation $(\mathrm{f}=80 \mathrm{~Hz}, 160 \mu \mathrm{s})$ geprüft.

Eingeschlossen wurden 30 gesunde Probanden im Alter von 18-28 Jahren und 10 Patienten mit chronischem Rückenschmerz im Alter von 1965 Jahren.

Die individuellen Schwellen der QST für Kälte (CDT), Wärme (WDT), Temperaturunterschied (TSL), Kälte- (CPT) und Hitzeschmerz (HPT), Berührungswahrnehmung (MDT), Spitz/Stumpf-Diskriminierung (MPT), Wind up Ratio (WUR) sowie Druckschmerz (PPT) wurden bestimmt. Lokalisiert am Handrücken wurde die Testung beidseits ohne und unter nicht spürbarem Reizstrom von 1mA (TENS1) durchgeführt. Appliziert wurde TENS ausschließlich am linken Arm. Im Abstand erfolgten Messwiederholungen mit einer Parästhesie induzierenden Stromstärke von 6-8mA (TENS2).

Ergebnisse: Für beide Kollektive zeigten sich signifikante Unterschiede $(p<0,05)$ unter TENS2 für alle Parameter der QST sowohl über der Applikationsseite als auch kontralateral. Lediglich die WUR ergab kontralateral zum Applikationsort in der Patientengruppe keinen signifikanten Messunterschied.

Bei den Gesunden zeigte die MDT die größte Schwellenzunahme, bei den chronischen Schmerzpatienten die WDT.

Bereits unter TENS1 fiel eine reduzierte Empfindung von Kalt-, Warmreizen sowie von taktilen und Druckschmerzreizen im Applikationsareal bei Probanden und geringer bei Schmerzpatienten auf. Kontralateral zeigten Probanden unter TENS1 eine erhöhte Schwelle für Kälte- und taktile Reize, bei Schmerzpatienten ließen sich hier keine Unterschiede zur Kontrollmessung nachweisen.

Schlussfolgerung: TENS erhöht abhängig von der Impulsstärke die Schwellen sensorischer Wahrnehmung am Applikationsort sowie in geringerem Ausmaß kontralateral. Die Ergebnisse weisen auf Unterschiede zwischen gesunden Probanden und chronischen Schmerzpatienten hin. Annehmbar beeinflussen Faktoren wie Alter, Begleiterkrankungen, Pharmaka und Sensibilisierungsmechanismen die sensorischen TENS-Effekte.

\section{P02.02 - Die Wirkung von transkutaner Vagusnervstimulation (TVNS) auf die Wahrnehmung von wiederholten schmerzhaften Hitze-Reizen - eine randomisierte kontrollierte Crossover-Studie}

H. Hacker', N. Möller', K. Hahnenkamp' ', T. Usichenko'

'Universitätsmedizin Greifswald, Klinik für Anästhesiologie, Greifswald, Deutschland
Hintergrund: Transkutane Vagusnervstimulation ist eine vielversprechende Methode zur Behandlung von akuten und chronischen Schmerzen. Experimentelle Effektivitätsuntersuchungen von TVNS führten bisher jedoch zu kontroversen Ergebnissen. Ziel dieser Studie war es, den Einfluss von TVNS auf die Wahrnehmung von wiederholten schmerzhaften Hitze-Reizen bei gesunden Probanden im Vergleich mit Sham- und Placebo-Stimulation zu untersuchen. Methode: 49 Probanden (25 Frauen) nahmen an vier (crossover) Untersuchungen mit einem Abstand von mind. $48 \mathrm{~h}$ teil. Während jeder Untersuchung wurden 90 Hitze-Reize mit Schmerztoleranztemperatur (Ramp Rate: $40^{\circ} \mathrm{C} / \mathrm{s}$, Pulsplateaudauer: $0,8 \mathrm{~s}$, Frequenz: 0,33 Hz) mit einer CHEPS-Thermode (Medoc, Israel) am Unterarm appliziert. Die erste Untersuchung wurde ohne Intervention durchgeführt (Baseline), in drei folgenden wurde randomisiert entweder TVNS, Sham- (Stimulation am Ohrläppchen) oder Placebo-Stimulation (inaktives Gerät) 20 min vor und während der Hitze-Reizung durchgeführt. Die Probanden bewerteten die Schmerzintensität des ersten und jeden 10. Stimulus auf einer numerischen Skala $(0-100)$. Die Datenanalyse erfolgte mittels mixed-model ANOVA und Bonferroni adjustierten multiplen Vergleichen. Ergebnisse: Die wahrgenommene Schmerzintensität der Reize war während aller Interventionen im Vergleich zur Baseline deutlich gesenkt $(p<.001)$. Die Schmerzintensität beim 30. Stimulus war unter TVNS signifikant geringer als unter Placebo-Stimulation $(p=.038)$. Die Schmerzintensitäten unter TVNS und Sham-Stimulation waren nicht zu unterscheiden. Konklusion: In dieser randomisierten verblindeten crossover Studie konnten schmerzlindernde Effekte von elektrischer TVNS gezeigt werden. Diese Effekte waren zu den meisten Zeitpunkten nicht von denen in der Placebo- und Sham-Bedingung zu unterscheiden.

\section{P02.03 - Feedbacktraining des nozizeptiven Rückziehreflexes bei chronischen Rückenschmerzpatienten: Einfluss auf klinischen Schmerz und Conditioned Pain Modulation}

\section{S. Krafft' , S. Krafft ${ }^{2}$, H. Göhmann ${ }^{3}$, J. Sommer', A. Straube', A. Straube ${ }^{2}$, R.} Ruscheweyh ${ }^{1}$

'Ludwig-Maximilians-Universität München, Neurologische Klinik und Poliklinik, Großhadern, München, Deutschland, ${ }^{2}$ Ludwig-MaximiliansUniversität München, Graduate School of Systemic Neurosciences, München, Deutschland, ${ }^{3} \mathrm{Klinikum}$ Traunstein, Abteilung für Anästhesie, Intensivmedizin und Schmerztherapie, Traunstein, Deutschland, ${ }^{4}$ PhilippsUniversität Marburg, Klinik für Psychiatrie und Psychotherapie, Marburg, Deutschland

Einleitung: Chronische Schmerzpatienten weisen eine Unterfunktion der absteigenden Schmerzhemmung auf, die vermutlich zur Persistenz des Schmerzes beiträgt. Ein gezieltes Training zur Verbesserung der absteigenden Schmerzhemmung bildet daher einen attraktiven Ansatz in der Schmerztherapie. Die absteigende Schmerzhemmung reduziert durch Serotonin- und Noradrenalinausschüttung die spinale Nozizeption und somit den nozizeptiven Zustrom zum Gehirn. Ihre Aktivität wird durch kognitiv-emotionale Prozesse moduliert. In einem zuvor von uns entwickelten Feedbacktraining erlernten gesunde Probanden Kontrolle über ihre Schmerzhemmung, indem sie kognitiv-emotionale Strategien anwendeten und Rückmeldung über ihren nozizeptiven Rückziehreflex (RIIIReflex), einem Maß der spinalen Nozizeption, erhielten. Hier wurde untersucht, ob chronische Schmerzpatienten durch dieses Feedbacktraining ihre absteigende Schmerzhemmung verbessern und damit die klinischen Schmerzen verringern können.

Methoden: Der RIII-Reflex wurde durch elektrische Stimulation des N. suralis ausgelöst und die RIII-Reflexgröße den Probanden unmittelbar auf einem Bildschirm angezeigt. Auf Kommando sollten die Probanden kognitiv-emotionale Strategien verwenden, um die Reflexgröße zu reduzieren. Chronische Rückenschmerzpatienten erhielten wahres $(n=18)$ oder falsches (sham, $n=15$ ) Feedback (FB), gesunde Kontrollen erhielten wahres FB $(n=15)$. Vor und nach dem RIII-Feedbacktraining wurde der klinische Rückenschmerz anhand der Numerischen Rating Skala (NRS [0 - 10]) bewertet und die absteigende Schmerzhemmung mittels Conditioned Pain Modulation (CPM)-Tests gemessen. Eine signifikante Reduktion eines 
Testreiz-Schmerzes (schmerzhafter Hitzereiz) bei gleichzeitiger Anwendung eines konditionierenden Reizes (schmerzhaftes Kaltwasserbad) zeigt hierbei eine gute Aktivierbarkeit der absteigenden Hemmung.

Ergebnisse: Alle drei Gruppen erreichten eine signifikante RIII-Reflexreduktion. Vor dem Training zeigten die Patienten beim CPM-Test kaum Schmerzreduktion (wahres FB: auf $98 \pm 26 \%$; Sham-FB auf $87 \pm 25 \%$, jeweils n.s.), während die Kontrollen einen signifikanten CPM-Effekt zeigten (auf $81 \pm 22 \%, p<0,01$ ). Nach dem Training gab es in allen Gruppen einen signifikanten CPM-Effekt (Patienten mit wahrem FB: auf $80 \pm 21 \%$; Patienten mit Sham-FB: auf $81 \pm 22 \%$; Kontrollen: auf $83 \pm 31 \%$, alle $p<0,05)$, jedoch erreichten nur Patienten mit wahrem FB eine signifikante Verbesserung des CPM-Effekts im Vergleich zu vor dem Training $(p<0,01)$. Ebenso zeigten nur Patienten mit wahrem FB nach dem Training eine signifikante Verringerung ihres chronischen Rückenschmerzes (maximaler Schmerz: von 7,1 $\pm 1,7$ auf 5,7 $\pm 2,0[0-10], p<0,01$ ).

Diskussion: Die Daten legen nahe, dass das RIII-Feedbacktraining die absteigende Schmerzhemmung, gemessen am CPM-Effekt, und den klinischen Schmerz bei chronischen Schmerzpatienten signifikant verbessern kann. Das RIII-Feedbacktraining könnte daher einen nicht-pharmakologischen Beitrag in der Schmerztherapie leisten.

\section{P02.04 - Visuelles Feedback verbessert den Effekt klassischer Massagetherapie bei Patienten mit chronischen Rückenschmerzen.}

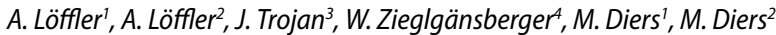

${ }^{1}$ Ruhr-Universität Bochum, Klinik für Psychosomatische Medizin und Psychotherapie, LWL-Universitätsklinkum, Bochum, Deutschland, ${ }^{2}$ Universität Heidelberg, Institut für Neuropsychologie und Klinische Psychologie, Zentralinstitut für Seelische Gesundheit Mannheim, Medizinische Fakultät Mannheim, Mannheim, Deutschland, ${ }^{3}$ Universität Koblenz-Landau, Institut für Psychologie, Landau, Deutschland, ${ }^{4}$ Max Plack Institut für Psychiatrie, Institut für klinische Neuropharmakologie, München, Deutschland

Hintergrund:Empirische Befunde zeigen, dass die Visualisierung chronisch schmerzender und schmerzhaft stimulierter Körperteile schmerzhemmend wirkt. In dieser Studie wurde untersucht, ob die Visualisierung des Rückens während einer klassischen Massage, den Effekt der Behandlung auf den chronischen Schmerz bei Patienten mit Rückenschmerz verstärkt.

Methode:19 Patienten mit chronischen Rückenschmerzen wurden mit klassischer Massagetherapie behandelt. Während der Behandlung erhielten die Patienten ein visuelles Feedback des Rückens und konnten so die Massage in Echtzeit nachverfolgen. Als Kontrollbedingungen diente der Blick auf ein unbewegtes Bild des eigenen Rückens, auf eine Rückenmassage einer fremden Person, auf einen neutralen Gegenstand und eine Massage mit geschlossenen Augen. Die Bedingungen wurden in einem withinsubject Design von jedem Probanden in randomisierter Reihenfolge an fünf unterschiedlichen Tagen durchlaufen.

Ergebnisse:In allen Bedingungen reduzierte sich der habituelle Schmerz signifikant. Das visuelle Feedback des eigenen Rückens hatte dabei den stärksten Effekt und unterschied sich signifikant von dem Feedback eines neutralen Gegenstandes. Zu den anderen Kontrollbedingungen gab es keine signifikanten Unterschiede.

Schlussfolgerung: Möglicherweise haben die weniger spezifischen Kontrollbedingungen ebenfalls einen Effekt weil der Blick auf das Standbild des eigenen Rückens und auf eine Massage einer fremden Person auch ein Zugehörigkeitsgefühl (ownership) induzieren. Die Ergebnisse legen nahe, dass visuelles Feedback in Echtzeit hilfreich sein könnte um die Effekte von Behandlungstechniken bei chronischen Schmerzpatienten zu verbessern. Diese Studie wurde durch einen Early Career Research Grant der International Association for the Study of Pain und der Deutsche Forschungsgemeinschaft DI 1553/3 gefördert.

\section{P02.05 - Sensorische Mapping der durch die okzipitale} Nervenstimulation (ONS) induzierten räumlichen, kognitiven und affektiven Empfindungen in der Behandlung der chronischen Migräne

A. Göbel', C. Göbel', U. Niederberger'², A. Heinze ${ }^{3}$, K. Heinze-Kuhn ${ }^{3}$, H. Gergely3, C. Meinecke ${ }^{3}$, S. Clasen ${ }^{3}$, H. Mehdorn ${ }^{4}$, D. Rasche ${ }^{5}$, H. Göbel ${ }^{3}$

${ }^{1}$ Klinik für Neurologie, Universitätsklinikum Schleswig Holstein, Lübeck, Deutschland, ${ }^{2}$ Universitätsklinikum Schleswig-Holstein, Campus Kiel, Institut für Medizinische Psychologie Kiel, Kiel, Deutschland, ${ }^{3}$ Schmerzklinik Kiel, Klinik für neurologisch-verhaltensmedizinische Schmerztherapie, Kiel, Deutschland, ${ }^{4}$ Klinik für Neurochirurgie, Universitätsklinikum Schleswig Holstein, Kiel, Deutschland, ${ }^{5}$ Klinik für Neurochirurgie, Universität zu Lübeck, Lübeck, Deutschland

Einleitung: Die okzipitale Nervenstimulation (ONS) wird zur Behandlung der therapieresistenten chronischen Migräne eingesetzt. Die Programmierung der zu verwendenden Stimulationsparametern, sowie die Einstellung der induzierten räumlichen, kognitiven und affektiven Empfindungen erfolgte bisher ohne empirische Grundlagen. Ziel der Studie war daher die Verbindung zwischen Stimulationsparametern, den induzierten Empfindungen sowie den klinischen Behandlungseffekten durch sensorisches Mapping zu analysieren.

Methodik: Es wurden 32 Patienten in die Analyse einbezogen (25 Frauen, 7 Männer, Alter 45,77 $\pm 11,26$ Jahre, Spannweite 22-67 Jahre). Die Patienten litten alle an therapierefraktärer chronischer Migräne über eine mittlere Zeitdauer von 21,35 $\pm 8,05$ Jahren (Spannweite 10-46 Jahre) und wurden durch die ONS behandelt. Die Follow-up-Periode erstreckte sich über den Zeitraum September 2013 bis Juni 2015. Zur Bewertung des Zusammenhangs zwischen den Stimulationsparametern, den dadurch induzierten Empfindungen und den klinischen Behandlungseffekten wurde eine neue computerbasierende bildgebende Methode zur Erfassung der räumlichen, kognitiven und affektiven sensorischen Effekte der ONS eingesetzt [1] Während des Beobachtungszeitraumes wurden insgesamt 808 individuelle quantitative und qualitative Datensätze analysiert.

Ergebnisse: $48,9 \%$ der Patienten berichteten, dass der globale Effekt der ONS-Behandlung moderat bis sehr gut war. $44,6 \%$ berichteten einen schwachen Effekt der ONS und 6,1\% berichteten keinen therapeutischen Effekt. Patienten die durch die ONS-induzierte Empfindungen oberhalb der Linie zwischen den Meatus acusticus externus und der Protuberantia occipitalis externa (MOP-Linie) verspürten, berichteten einen hochsignifikant besseren Behandlungseffekt im Vergleich zu den Patienten, bei denen die Empfindungen unterhalb dieser Linie induziert wurden ( $p=0,0000001)$. Empfindungen unterhalb der MOP-Linie führten nicht zu signifikanten Behandlungseffekten. Frauen und Patienten mit chronischer Migräne, die zusätzlich an hochfrequenten Kopfschmerz vom Spannungstyp litten, profitierten mit höherer Wahrscheinlichkeit durch die ONS-Behandlung $(p=0,000005)$. Wenn die durch die ONS induzierten Empfindungen einen kneifenden, pulsierenden oder pochenden Charakter aufwiesen, waren die therapeutischen Ergebnisse hochsignifikant besser als bei Probanden, die andere Empfindungen verspürten $(p=0,000001)$.

Schlussfolgerung: In dieser Studie führte die Behandlung der chronischen Migräne durch die occipitale Nervenstimulation zu hochsignifikanten Langzeiteffekten. Die Platzierung der ONS-Elektroden sollte oberhalb der MOP-Linie erfolgen um ein räumliches Empfindungsfeld über dieser Linie zu realisieren. Die spezifische Programmierung der Stimulationsparameter mit gezielter Anpassung der kognitiven und affektiven Empfindungsparameter kann hochsignifikant die Therapieeffekte der ONS in der Behandlung der chronischen Migräne verbessern.

\section{Literatur}

1. Göbel et al (2015) Imaging Sensory Effects of Occipital Nerve Stimulation: A New

Computer-based Method in Neuromodulation. Brain Stimul 8(2):295-298 


\section{P03 - Experimentelle Schmerzmodelle (Mensch)}

\section{P03.01 - Die neuronalen Korrelate der Störfunktion von Schmerz an Gesicht und Hand und der Einfluss schmerzbezogener Angst}

\author{
K. Schmidt ${ }^{1}$, K. Forkmann ${ }^{1}$, C. Sinke ${ }^{2}$, U. Bingel ${ }^{1}$ \\ 'Universitätsklinikum Essen, Klinik für Neurologie, Essen, Deutschland, \\ ${ }^{2}$ Medizinische Hochschule Hannover, Hannover, Deutschland
}

Gesichtsschmerz führt im Vergleich zu Schmerz an den Extremitäten zu erhöhter Angst. Es wird vermutet, dass diese erhöhte Angst und die hohe biologische Relevanz von Kopf- und Gesichtsschmerz mit einer höheren Störfunktion von Schmerzen im Gesicht auf kognitive Prozesse in Zusammenhang stehen. In dieser fMRT Studie untersuchten wir den Einfluss von Gesichts- und Handschmerz auf visuelle Verarbeitung und Gedächtnisenkodierung auf neuronaler und Verhaltensebene. 23 junge gesunde Probanden absolvierten eine visuelle Kategorisierungsaufgabe unter gleichzeitiger Applikation von elektrischen Schmerzreizen an Hand oder Gesicht. Im Anschluss folgte eine unangekündigte Gedächtnisaufgabe. Die Ergebnisse dieser Studie können die erhöhte Angst für Gesichts- ggü. Handschmerz bestätigen. Kategorisierungs- und Erinnerungsleistung unterschieden sich auf der Verhaltensebene nicht voneinander. Auf neuronaler Ebene jedoch zeigte sich erhöhte Aktivität in aufgabenrelevanten gedächtnisbezogenen und visuellen Arealen, wie z.B. dem Hippocampus und lateralten occipitalen Komplex (LOC), für Bilder, die mit Gesichtsschmerz gepaart wurden ggü. Bildern, die mit Handschmerz gepaart wurden. Darüber hinaus zeigte sich ein Zusammenhang für eine stärkere Gedächtnisenkodierung im Hippocampus mit erhöhter schmerzbezogener Angst für Gesichtsim Vergleich zu Handschmerz. Die funktionelle Konnektivität zwischen Amygdala und LOC war erhöht für Gesichts- ggü. Handschmerz. Die Befunde lassen auf angstgesteuerte kompensatorische Ressourcenaktivierung, verstärkt für Gesichtsschmerz, schließen und sind ein weiterer Hinweis auf die besondere biologische Relevanz von Gesichtsschmerz und die damit verbundene klinische Bedeutung von Kopf- und Gesichtsschmerzerkrankungen.

\section{P03.02 - Wahrnehmung von wiederholten schmerzhaften Hitze-Reizen durch gesunde Probanden - Vergleich von vier Kombinationen verschiedener Stimulationsparameter}

\section{N. Möller' ${ }^{1}$, H. Hacker' ${ }^{1}$ K. Hahnenkamp ${ }^{1}$, T. Usichenko \\ 'Universitätsmedizin Greifswald, Klinik für Anästhesiologie, Greifswald, Deutschland}

Hintergrund: Um klinische (chronische) Schmerzerfahrungen valider imitieren zu können, sollte die Zunahme der wahrgenommenen Intensität von wiederholten schmerzhaften Reizen (temporale Summton von Schmerz; TSP) Gegenstand experimenteller Untersuchungen sein. Ziel dieser Pilotstudie war es, eine Kombination von Stimulationsparametern zu finden, mit denen das TSP-Phänomen zuverlässig bei gesunden Probanden induziert werden kann.

Methode: Zwei Stichproben mit je 20 gesunden Probanden (15 Frauen) wurden in insgesamt 4 Untersuchungen mit pulsierenden Hitze-Reizen (Frequenz: 0,33 Hz, Ramp Rate: $40^{\circ} \mathrm{C}$ ) stimuliert, die mit einer CHEPSThermode (Medoc, Israel) am linken Unterarm appliziert wurden. In den Untersuchungen I bis IV wurden die Stimulationstemperatur (I und II: Schmerzschwelle $+0,5^{\circ} \mathrm{C}$, III: Schmerzschwelle $+1,0^{\circ} \mathrm{C}$, IV: Schmerztoleranz), die Anzahl der Hitze-Reize (I-III: 60, IV: 90) und die Dauer des Puls-Plateaus (I: 500 ms, II und IV: 800 ms, III: 1000 ms) variiert. Die Probanden bewerteten die Schmerzintensität des ersten und jeden 10. Stimulus auf einer numerischen Skala (0-100). Eine Stichprobe absolvierte Untersuchungen I bis III in einem crossover Design, die 2. Stichprobe absolvierte Bedingung IV. TSP-Anstiege wurden als Differenz zwischen dem niedrigsten und dem letzten Rating berechnet und mittels Wilcoxon- bzw. Man-Whitney-U-Tests verglichen. Nur $\mathrm{P}<0,01$ gelten als signifikant, um für multiple Vergleiche zu korrigieren.

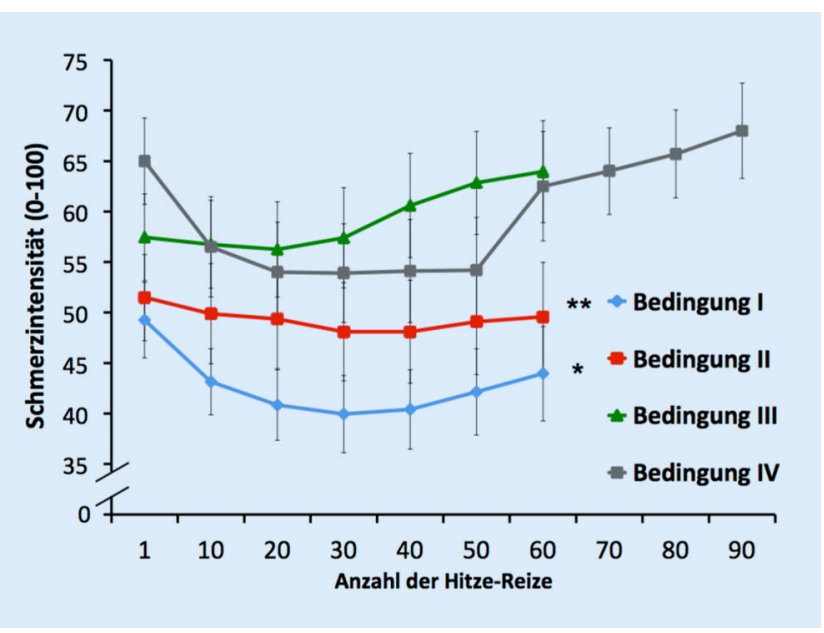

Abb. 1 A

Ergebnisse: Der größte TSP-Anstieg ergab sich in Bedingung IV (Median $(\mathrm{Mdn})=17,5)$, dieser war signifikant größer als die TSP-Anstiege in den Bedingungen I ( $\mathrm{Mdn}=5, p=.002)$ und II ( $\mathrm{Mdn}=5, p=.001$; $\bullet$ Abb. 1$)$, jedoch nicht signifikant größer als in Bedingung III (Mdn $=10)$. In Bedingung III berichteten 3 Personen über nicht auszuhaltende Schmerzen zum Ende der Hitze-Stimulation.

Schlussfolgerung: Bedingung IV konnte das TSP Phänomen am zuverlässigsten und bei den meisten Probanden der Stichprobe erzeugen. Stimulationsparameter in den anderen Bedingungen riefen entweder zu geringe TSPAnstiege hervor oder wurden von einigen Probanden als „nicht zu ertragen“ beschrieben. Reliabilität und Validität einer Methode mit den Parametern aus Bedingung 4 müssen nun in größeren Stichproben geprüft werden.

\section{P03.03 - Antizipation vs. Realisierung eines 2-h-Ausdauerlaufes: Was wirkt schmerzhemmender?}

M. Carl' ', L. Eichelkraut ${ }^{1}$, W. Miltner' ${ }^{1}$, T. Weiss ${ }^{1}$

'Institut für Psychologie, Lehrstuhl für Biologische und Klinische Psychologie, Jena, Deutschland

Hintergrund: Neueste Studien lassen vermuten, dass endogene Opioide nicht nur für die euphorisierende Wirkung von Ausdauersport, sondern auch für das Phänomen der sportbedingten Analgesie eine wesentliche Rolle spielen können. Die genauen Wirkmechanismen, insbesondere die Funktionen der daran beteiligten Gehirnregionen, sind allerdings bisher kaum erforscht. Ziel dieser Arbeit war es daher, die Auswirkungen eines 2-h-Ausdauerlaufes auf die Verarbeitung schmerzhafter Stimulationen im Gehirn zu untersuchen. Als erste Studie überhaupt wurde dabei ein besonderes Augenmerk auf die Effekte kurz vor Beginn, also in Antizipation des Laufes, gelegt. Methode: Hierfür wurde die Schmerzverarbeitung an 13 erfahrenen Ausdauerläufern direkt vor und direkt nach einem 2-h-Ausdauerlauf sowie an einem Ruhetag, an dem nicht gelaufen werden sollte, mittels funktioneller Magnet-Resonanz-Tomographie (fMRT) untersucht. Auch die Euphorie der Teilnehmer wurde jeweils erfasst.

Ergebnisse: Eine veränderte Schmerzverarbeitung nach dem 2-h-Experimentallauf, einhergehend mit einem Euphorie-Erlebnis, konnte weder in den Verhaltensdaten, noch mit den fMRT-Befunden repliziert werden. Jedoch deuten die fMRT-Ergebnisse darauf hin, dass die Schmerzverarbeitung in Antizipation des Laufes im Vergleich zum Ruhetag verändert ist. So fanden sich während der Schmerzstimulation eine erhöhte Aktivität im medialen Präfrontalkortex (mPFC) vor dem Lauf im Vergleich zur Ruhemessung sowie verminderte Aktivitäten in zwei Clustern der posterioren Insula. Diese veränderten Aktivitäten in den opioidergen Gehirnregionen der Neuromatrix des Schmerzes deuten auf eine verminderte zentrale Schmerzverarbeitung in Antizipation des Laufes hin. Außerdem konnte in einer 
Korrelationsanalyse gezeigt werden, dass die Stärke der Veränderung der Euphorie vor dem Lauf verglichen zur Messung am Ruhetag mit der Veränderung der Aktivität einiger opioiderger Regionen $(\mathrm{mPFC} /$ pregenualer anteriorer cingulärer Kortex (pACC)) während der Schmerzstimulation im Gehirn zusammenhängt.

Schlussfolgerung: Die Ergebnisse weisen darauf hin, dass bereits die Antizipation eines Ausdauerlaufes ausreichend ist hypoalgetische Effekte, möglicherweise über eine Aktivierung des endogenen opioiderg vermittelten Schmerzhemmungssystems, auszulösen. Weiterführende (PET)-Studien sollten diese Vermutung prüfen.

\section{P03.04. - Periphere und zentrale Komponenten der veränderten Hitzeschmerzwahrnehmung während repetitiver Laserreizung bei gesunden Probanden}

D. Rosenberger', S. Schuh-Hofer', W. Magerl', R. Treede', W. Greffrath ${ }^{1}$

${ }^{1}$ Medizinische Fakultät Mannheim der Universität Heidelberg,

Mannheim, Lehrstuhl für Neurophysiologie, Zentrum für Biomedizin und

Medizintechnik Mannheim, Mannheim, Deutschland

Hintergrund: Wiederholte Hitzeschmerzreize können in Abhängigkeit von Reizbedingungen und -kontext (z. B. Interstimulusintervall (ISI) bzw Reizfrequenz) zu gesteigerter oder verringerter Schmerzempfindung führen („Dual Process Theory“). Während auf peripherer Nozizeptor-Ebene die regelhaft beobachtete abnehmende Reizantwort auf repetitive Reizung als „Fatigue“ oder „Tachyphylaxie“ bezeichnet wird, bezieht sich „Habituation" auf zentrale Prozesse der Schmerzverarbeitung.

Ziel dieser Studie war es, periphere (Fatigue/Tachyphylaxie) und zentrale (Habituation) Komponenten der Schmerzhaftigkeit repetitiver Laserhitzereize bei gesunden Probanden zu charakterisieren.

Methoden: Bei gesunden Probanden $(n=10)$ wurden mit einem Nahinfrarot-Diodenlaser ( $1475 \mathrm{~nm}, 100 \mu \mathrm{m}$ Strahldurchmesser) Reizserien von 5 Reizen (S1-S5, 100 mW, 200 ms Reizdauer) mit unterschiedlichen ISIs (0,3, 1, 3, 10 und 30 s) auf den Fußrücken appliziert. Diese Reizmuster wurden nach 2 und 20 Minuten wiederholt. Der Reizort wurde zwischen den einzelnen Reizen und/oder den Reizserien entweder verändert (Ortswechsel, d.h. überwiegend Habituation) oder aber gleich belassen (kein Ortswechsel, d.h. Fatigue und Habituation). Erhoben wurden individuelle Schmerzratings auf S1-S5 (NRS 0 - 100, kein Schmerz - maximal vorstellbarer Schmerz). Resultate: Bei Gesunden zeigte sich bei repetitiver Laserhitzereizung ohne Ortswechsel mit ISIs von $=3 \mathrm{~s}$ eine deutliche Abnahme des Hitzeschmerzes um bis zu $86 \%$ von S1 bis S5 (ISI $10 \mathrm{~s}, p<0,001$ ). Dieser Effekt war wesentlich schwächer ausgeprägt bei variablen Reizorten (maximale Reduktion um $20 \%$ (n.s. $p>0,2$ ) und Tendenz zur Zunahme der Ratings (z. B. um $88,5 \%$ bei ISI $10 \mathrm{~s}, p<0,001$ )). Die stärkste Abnahme der Ratings (ohne Ortswechsel) erfolgte zwischen S1 und S2 um 71,6\%, vereinbar mit dem Tachyphylaxieverlauf bei isoliert gemessenen nozizeptiven Neuronen [1]. Bei ISIs $<3$ s zeigte sich tendenziell eine Zunahme der Ratings (zeitliche Summation, bei Ortswechsel stärker ausgeprägt). Bei der Wiederholung der Reizserien nach 2 Minuten zeigte sich ohne Ortswechsel ISI-abhängig eine partielle Erholung von der Tachyphylaxie der vorhergehenden Serie (mittlere verbleibende Reduktion der S1-Ratings im Vergleich zur ersten Serie 35,5 $\pm 12,6 \%)$. Die Tachyphylaxie (S5:S1) innerhalb der zweiten Reizserie blieb im Vergleich zur ersten gleich. Mit Ortswechsel ergab sich nach zwei Minuten eine mittlere Reduktion der jeweils ersten Werte um $26,9 \pm 29,8 \%$ ohne Korrelation mit dem ISI. Nach 20 Minuten fand sich eine nur partielle Erholung der Schmerzratings in beiden Paradigmen. Schlussfolgerung. Bei wiederholter Laserhitzereizung desselben Ortes trägt periphere Nozizeptor-Fatigue bei ISIs $=3 \mathrm{~s}$ maßgeblich zur Abnahme des subjektiven Schmerzempfindens bei und übertrifft dabei den Grad der zentralen Habituation. Höherfrequente Reizung bewirkt dagegen eine Verstärkung des Hitzeschmerzes.

\section{Literatur}

1. Schwarz S et al (2000) Inactivation and tachyphylaxis of heat-evoked inward currents in nociceptive primary sensory neurones of rats. Journal Physiology-London 528(3):539-549
P03.05 - Einfluss einer Habituation auf Schmerzintensität und schmerzevozierte Potentiale (PREP) im Vergleich zu CPM-Effekten

\section{Eitner' ', C. Maier' ', Ö. Özgül'², E. Enax-Krumova², J. Vollert' ${ }^{1}$, N. Kumowski',} O. Höffken ${ }^{2}$

'Berufsgenossenschaftliches Universitätsklinikum Bergmannsheil $\mathrm{GmbH}$, Bochum, Abteilung für Schmerztherapie, Bochum, Deutschland, ${ }^{2}$ Berufsgenossenschaftliches Universitätsklinikum Bergmannsheil GmbH, Neurologische Universitätsklinik und Poliklinik, Bochum, Deutschland

Hintergrund: Ziel der aktuellen Studie war zu untersuchen, ob sich bei repetitiver 30-minütiger Applikation von mittelstark schmerzhaften elektrischen Reizen auf der Haut durch Habituationseffekte die Schmerzintensität sowie elektrophysiologische Parameter der schmerzevozierten Potentiale (PREP) systematisch verändern und ob hierfür Lerneffekte bedeutsam sind. Zudem wird wurde untersucht, ob das Ausmaß der Habituationseffekte jene Schmerz- und Amplitudenveränderungen erklären, die durch einen zweiten kontralateral applizierten Kälteschmerz gemäß dem Conditioned Pain Modulation-Protokolls (CPM) 1 ausgelöst wurden. Methodik: Bei 30 Gesunden (12 männlich; Alter 20-35 Jahre) erfolgte die Reizung über drei triangulär platzierte K2-Stimulationselektroden im Versorgungsgebiet des $\mathrm{N}$. radialis superficialis in einer Schmerzintensität mit dem Zielwert 50-60 (NRS 0-100) in Einzelblöcken, bestehend aus je 20 Triple-Reizen 2 . Nach jeweils vier Triple-Reizen wurde die aktuelle Schmerzeinschätzung erfragt. Alle Probanden erhielten eine Reizfolge mit konstanten Reizintensitäten (14 Minuten in 4 Blöcken). In Gruppe A wurde die Reizintensität anschließend um $75 \%, 50 \%$ und $25 \%$ randomisiert variiert, in Gruppe B in umgekehrter Reihenfolge (variable vor konstanter Reizserie). Nach den Reizserien erfolgte die CPM-Messung für beide Gruppen mit dem elektrischen Reiz als Teststimulus und einem kontralateralen Kaltwasserreiz $\left(10^{\circ} \mathrm{C}\right)$ als konditionierenden Stimulus. Die kortikale Ableitung der PREP erfolgte mit einer über $\mathrm{Cz}$ angebrachten Kontaktelektrode, die gegen die verlinkten Ohrelektroden referenziert wurde. Neben der Schmerzintensität wurden aus PREP-Kurven die N1und P1-Latenzen sowie die N1-P1-Amplituden ermittelt. Amplituden und Schmerzintensitäten wurden innerhalb der Individuen mittels gepaarter und zwischen den Verfahren mittels ungepaarter t-tests verglichen.

Ergebnisse: Nach konstanter Reizung zeigte sich ein Abfall der Schmerzintensität im Mittel um 14\% $(p<0,01)$, dagegen durch CPM um $37 \%$ $(p<0,001$, Unterschied Habituation vs. CPM $p<0,01)$. Die N1-P1-Amplituden fallen signifikant um $9 \%$ ab $(p<0,05)$, unter CPM um $27 \%$ $(p<0,001$, Unterschied Habituation vs. CPM $p<0,05)$.

Schlussfolgerung: Bei längerer schmerzhafter elektrischer Reizung mit PREP-Elektroden tritt eine geringe, aber systematische, d.h. auch nicht durch Vorerfahrung beeinflussbare Habituation ein, die sich auch elektrophysiologisch durch einen geringen Abfall der Amplituden nachweisen lässt. Quantitativ sind diese Effekte aber deutlich geringer als die durch CPM mit diesem PREP-Kälteschmerzparadigma 3 erzielbare Reduktion der Schmerzintensität und PREP-Amplituden, so dass hierfür ein über das Maß der Habituation gehender Effekt angenommen werden muss.

\section{Literatur}

1. Yarnitsky D (2010) Conditioned pain modulation (the diffuse noxious inhibitory control-like effect): its relevance for acute and chronic pain states. Curr Opin Anaesthesiol 23:611-615

2. Obermann $\mathrm{M}$ et al (2008) Correlation of epidermal nerve fiber density with painrelated evoked potentials in HIV neuropathy. Pain 138:79-86

3. Höffken $\mathrm{O}$ et al (2016) A single dose of lorazepam reduces paired-pulse suppression of median nerve evoked somatosensory evoked potentials. Eur J Neurosci 43(9):1156-1160 
P03.06 - Einfluss von endogenem Testosteron auf sensorische und schmerzhafte Empfindungen, sowie die körpereigene Schmerzhemmung bei Männern

V. Deichert ${ }^{1}$, M. Wichmann ${ }^{1}$, M. Schubert ${ }^{2}$, M. Pereira', A. Soave ${ }^{3}$, C. Krallmann ${ }^{3}$, M. Zitzmann ${ }^{3}$, S. Kliesch', E. Pogatzki-Zahn ${ }^{1}$

'Universitätsklinikum Münster, Klinik für Anästhesiologie, operative Intensivmedizin und Schmerztherapie, Münster, Deutschland, ${ }^{2}$ Centrum für Reproduktionsmedizin und Andrologie, Abteilung für Klinische und Operative Andrologie, Münster, Deutschland, ${ }^{3}$ Centrum für Reproduktionsmedizin und Andrologie, Abteilung für Klinische und Operative Andrologie, Universitätsklinikum Münster, Münster, Deutschland

Hintergrund: Geschlechtsspezifische Unterschiede in der Wahrnehmung, Verarbeitung und Therapie von Schmerzen geraten zunehmend in den Fokus der Schmerzforschung. Dabei zeigt sich, dass Frauen häufiger unter chron. Schmerzerkrankungen leiden und die Schmerzempfindlichkeit eher höher ist als bei Männern [1]. Endogene Hormone wie Östrogen und/ oder Progesteron könnten einen Teil der stärkeren Schmerzempfindlichkeit von Frauen erklären [2]. Für die eher geringere Schmerzempfindlichkeit bei Männern wird vermutet, dass Testosteron das Schmerzempfinden möglicherweise vermindert.

Ziel: Untersuchung der Bedeutung endogener Testosteronspiegel für sensorische und schmerzhafte Reize sowie endogener Schmerzhemmung bei gesunden Männern.

Methodik: Bei gesunden Männern ( $n=47$, Alter: $31 \pm 11,49$ Jahre [MW \pm SD]) wurden mittels Quantitativer Sensorischer Testung (QST, Protokoll nach Rolke et al. 2006 [3]) psychophysiologische Wahrnehmungs- und Schmerzschwellen unterschiedlicher Modalitäten so wie Wind-Up und Schmerzempfindlichkeit auf Pinprickreize standardisiert untersucht. Im Anschluss wurde ein etabliertes CPM-Paradigma (Teststimulus[TS] = Hitzeschmerz [NRS ca. 60/100] wird durch zeitgleich applizierten konditionierenden Stimulus [CS] = Kälteschmerz [NRS ca. 60/100] gehemmt) durchgeführt. Abgeschlossen wurde jeder Testtermin mit einer Hormonbestimmung im Blut.

Auswertung: Es wurden 47 Männer eingeschlossen, davon 16 mit erniedrigtem $(<=10 \mathrm{nmol} / \mathrm{l})$ und 22 mit normwertigem Testosteron $(>=14 \mathrm{nmol} / \mathrm{l})$. Zwischen beiden Gruppen konnte kein sign. Unterschied in den Reizschwellen für sensorische oder schmerzhafte Reize gezeigt werden ( $p>0,05$ für alle Parameter aus der QST: CDT $[p=0,28]$, WDT $[p=0,06]$, TSL $[p=0,25], \mathrm{CPT}[p=0,87], \mathrm{HPT}[p=0,27], \mathrm{PPT}[p=0,90]$, MPT $[p=0,25]$, MPS [ $p=0,07]$, WUR $128[p=0,81]$, WUR256 [ $p=0,36]$, $\operatorname{MDT}[p=0,92]$, VDT $[p=0,80]$, je Mann-Whitney-U-Test). Die individuelle Ausprägung des CPM-Effekts zeigte keine sign. Korrelation zu der Konzentration von Testosteron im Blut $\left(\mathrm{r}^{2}=0,038 ; p=0,19\right)$. Sign. Korrelationen bestehen jedoch zwischen dem CPM-Effekt und sowohl dem Alter (Spearman's rho $=0,473 ; p<0,01$ ), als auch der Schmerzhaftigkeit des CS (Spearman's rho $=0,348 ; p<0,01$ ).

Diskussion: Die vermutete Korrelation zwischen dem endog. Testosteron und der Stärke des CPM-Effekts als Gradmesser endog. Hemmung konnte nicht bestätigt werden. Ebenso zeigten sich keine sign. Gruppenunterschiede hinsichtlich sensorischer und schmerzhafter Reize. Sign. Korrelation bestehen jedoch zwischen der Ausprägung des CPM-Effekts und dem Alter einerseits, sowie der Schmerzhaftigkeit vom CS andererseits. Die beiden letzten Ergebnisse sind vor beschrieben und unterstreichen die Validität der Ergebnisse [4, 5]. Diese vorläufigen Daten lassen vermuten, dass Testosteron keine Effekte auf Schmerzschwellen, überschwellige Hitzereize und endog.Hemmung hat. Eine Aufstockung der beiden Gruppen sowie weitere klinische Studien müssen weiteren Aufschluss über die Rolle von Testosteron auf Schmerz unter experimentellen Bedingungen bringen.

\section{P03.07 - Bedeutung der körpereigenen Schmerzhemmung für Hyperalgesie nach einer Verbrennung 1. Grades bei gesunden Probanden}

L. Hermeling, K. Mund, M. Pereira, V. Deichert, E. Pogatzki-Zahn

Universitätsklinikum Münster, Klinik für Anästhesiologie, operative Intensivmedizin und Schmerztherapie, Münster, Deutschland

Hintergrund: Ein großes Thema der letzten Jahre in der Schmerzforschung ist die Prädiktion und Prävention der Schmerzchronifizierung, die evtl. durch ein Missverhältnis von Hemmung und Fazilitierung/Sensibilisierung begünstigt wird. In klinischen Studien konnte ein Zusammenhang zwischen Sensibilisierung und Chronifizierung $[1,2]$ so wie ein gegensinniger Zusammenhang zwischen endogener Hemmung und Chronifizierung [3] angedeutet werden. Unklar ist, in wieweit endogene Hemmung und Sensibilisierung Prädiktoren für Chronifizierung darstellen.

Ziel/Hypothese: Untersuchung des Zusammenhangs zwischen endogener Hemmung (ermittelt via conditioned pain modulation, CPM) und zentraler Sensibilisierung nach einer Verbrennungsverletzung 1. Grades bei gesunden Probanden.

Methodik: Bei gesunden Männern wurde ein etabliertes CPM-Paradigma (Teststimulus, TS: Hitzeschmerzreiz am Unterarm [NRS 60/100], Konditionierender Stimulus, CS: Eintauchen der Hand in ein $10^{\circ} \mathrm{C}$ kaltes Wasserbad) durchgeführt; anschließend wurde eine $9 \mathrm{~cm}^{2}$ große Verbrennung 1. Grades am Unterschenkel der Probanden erzeugt und 1, 3, 9 und $24 \mathrm{~h}$ danach die Größe des mechanischen Hyperalgesie-Areals mittels eines von-Frey-Filaments $(256 \mathrm{mN})$ gemessen. Sensorische-/und Schmerzreizschwellen im Rahmen der Quantitativ Sensorischen Testung (QST) wurden vor und nach Verbrennung in dem Areal der Verbrennung und in $1 \mathrm{~cm}$ Abstand dazu gemessen. Die kontralaterale Seite diente als Kontrollareal. Auswertung: Untersucht wurden 34 Männer (23,35 $\pm 2,99$ Jahre, MW \pm SD). Die Hyperalgesieareale betrugen $1 \mathrm{~h}$ nach Auslösen der Verbrennung 45,68 $\mathrm{cm}^{2} \pm 18,41 \mathrm{~cm}^{2}(p<0,0001)$ und sanken auf $33,46 \pm 16,54 \mathrm{~cm}^{2}$ $(p<0,0001)$ nach $3 \mathrm{~h}, 20,94 \pm 13,55 \mathrm{~cm}^{2}(p<0,0001)$ nach $9 \mathrm{~h}$ und $0 \pm 10,22 \mathrm{~cm}^{2}$ nach $24 \mathrm{~h}(p>0,05)$ ab. Vor Verbrennung zeigten die Probanden einen deutlichen CPM-Effekt; die Schmerzintensität auf den Hitzeschmerzreiz sank von NRS 49,29 $\pm 16,55$ (MW \pm SD) bei alleiniger Applikation auf NRS 34,91 $\pm 16,45$ bei gleichzeitiger Applikation mit dem CS $(p<0,0001)$. Der individuelle CPM-Effekt zeigte keine Korrelation zur Größe des Hyperalgesieareals $1 \mathrm{~h}$ nach Verbrennung $\left(\mathrm{r}^{2}=0,09 ; p>0,05\right)$. Diskussion: Die Probanden zeigten im Mittel einen guten CPM Effekt vor der Verbrennungsläsion. Nach der Verbrennung zeigten sich für $9 \mathrm{~h}$ $(p<0,0001)$ sign. vergrößerte Hyperalgesieareale mit einem Maximum $1 \mathrm{~h}$ nach Verletzungsinduktion. Die Hypothese einer (negativen) Korrelation von CPM vor Verbrennung mit Hyperalgesiearealgröße nach Verbrennung konnte nicht bestätigt werden; dies lässt vermuten, dass eine verminderte endogene Hemmung mechanistisch nicht mit einer gesteigerten Hyperalgesie (gemessen an der Größe des Hyperalgesieareals) assoziiert ist. Ebenfalls damit angezweifelt werden kann der vormals an Patienten postulierte prädiktive Wert des CPM Effektes für Schmerzchronifizierung. Weitere Untersuchungen müssen an Probanden und Patienten die Bedeutung von endogener Hemmung für bestimmte Patientengruppen identifizieren und an Patienten mit hohen Fallzahlen den klinischen Zusammenhang verifizieren.

\section{P03.08 - Endogene Schmerzhemmung durch Schmerzlinderung als Belohnung}

S. Desch', M. Burdach', P. Schweinhardt'², H. Flor', S. Becker'

'Zentralinstitut für Seelische Gesundheit, Mannheim, Institut für Neuropsychologie und Klinische Psychologie, Mannheim, Deutschland, ${ }^{2}$ Alan Edwards Centre for Research on Pain, Montreal QC, Kanada

Hintergrund: Schmerzen lösen die Motivation aus, der schmerzhaften Situation zu entkommen bzw. den Schmerzzustand zu beenden. Kann eine Schmerzlinderung erzielt werden, wird dies wiederum belohnend, d.h. als angenehm und auch in Zukunft erstrebenswert erlebt. Von Schmerz und Belohnung ist bekannt, dass sie sich gegenseitig beeinflussen. Dies scheint 
sich z. B. auch in einer großen Überlappung funktioneller und struktureller Systeme unseres Gehirns, die an der Verarbeitung von Schmerz und Belohnung beteiligt sind, widerzuspiegeln. Die Mechanismen, die der Interaktion von Belohnung und Schmerz und insbesondere belohnender Schmerzlinderung zugrunde liegen, sind hingegen weitgehend unbekannt, obwohl davon auszugehen ist, dass ihnen eine große Bedeutung bei der Entwicklung und Aufrechterhaltung chronischer Schmerzen zukommt. Methode: Zehn gesunde Probanden nahmen an einer Pilot-Studie teil, in der die Effekte belohnender Schmerzlinderung auf die Schmerzwahrnehmung mit Schmerzreduktion ohne belohnende Aspekte verglichen wurde. Dazu spielten die Probanden ein Glücksradspiel während sie schmerzhafte Hitzereize an ihrem Arm erhielten. In der experimentellen Bedingung hatten sie die Möglichkeit, Schmerzlinderung zu gewinnen, während sie in der Kontrollbedingung die gleiche Schmerzreduktion erhielten, ohne einen Einfluss auf den Ausgang des Spiels zu haben. Unterschiede in der wahrgenommenen Schmerzintensität wurden auf einer visuellen Analogskala erfasst. Außerdem regulierten die Probanden in einem Verhaltensmaß die Stimulation selbst, um Habituation bzw. Sensibilisierung in Reaktion auf die Schmerzreize zu erheben.

Ergebnisse: Das Verhaltensmaß, nicht jedoch die Einschätzung der Schmerzintensität, deutet auf einen zusätzlichen schmerzhemmenden Effekt belohnender Schmerzlinderung im Vergleich zur einer reinen Reduktion der Schmerzintensität hin. In der experimentellen Bedingung zeigten neun von zehn Probanden eine im Vergleich zur Kontrollbedingung reduzierte Sensibilisierung in Reaktion auf das Nachlassen der thermischen Stimulation.

Ausblick: Die Ergebnisse verdeutlichen den motivationalen Aspekt von Schmerzlinderung. Die Suche nach Schmerzlinderung stellt einen grundlegende Motivation für Patienten mit chronischen Schmerzen dar. Der Einsatz von Schmerzlinderung im Sinne einer negativen Verstärkung könnte das Repertoire operanter Methoden erweitern, die bereits erfolgreich in der Behandlung chronischer Schmerzen eingesetzt werden. Der Beitrag wird unterstützt von folgenden Institutionen: Baden-Württemberg-Stiftung $\mathrm{GmbH}$.

\section{P03.09 - Die Rolle des Kleinhirns bei Akquisition, Extinktion und Reinstatement Schmerz-assoziierter Furcht - Eine fMRT- Untersuchung im viszeralen Schmerzmodell}

\section{F. Labrenz ${ }^{1}$, H. Claassen ${ }^{2}$, T. Ernst ${ }^{2}$, A. Icenhour ${ }^{3}$, J. Langhorst ${ }^{4}$, D. Timmann ${ }^{2}$,} S. Elsenbruch ${ }^{5}$

'Universitätsklinikum Essen, Institut für Medizinische Psychologie und Verhaltensimmunbiologie, Duisburg, Deutschland, ${ }^{2}$ Universitätsklinikum Essen, Klinik für Neurologie, Essen, Deutschland, ${ }^{3}$ Universitätsklinikum Essen, Institut für Medizinische Psychologie und Verhaltensimmunbiologie, Essen, Deutschland, ${ }^{4}$ Kliniken Essen-Mitte, Innere Medizin V, Naturheilkunde und integrative Medizin, Essen, Deutschland, ${ }^{5}$ Institut für Medizinische Psychologie, Verhaltensimmunbiologie, Essen, Deutschland

Die Beteiligung spezifischer Großhirnareale an assoziativen Lern- und Gedächtnisprozessen bei chronischen Schmerzen ist durch eine Vielzahl experimenteller Studien gut belegt. Jedoch gibt es erste Hinweise, dass das Kleinhirn ebenfalls kognitiv-emotionale Lernvorgänge vermittelt. Insbesondere funktionelle chronische Schmerzen wie beim Reizdarmsyndrom (RDS) sind durch emotionale Prozesse geprägt, die wesentlich zum Erund Verlernen Schmerz-assoziierter Furcht beitragen. Ziel dieser Studie war es, spezifisch die Kleinhirnareale zu analysieren, welche an der Akquisition und Extinktion Schmerz-assoziierten Furcht in einem klinischen, viszeralen Schmerzmodell beteiligt sind.

Insgesamt durchliefen 17 RDS-Patientinnen und 21 gesunde Probandinnen im 3T MRT eine differentielle Furchtkonditionierung, während der schmerzhafte rektale Distensionen (US) wiederholt mit einem visuellen Stimulus $\left(\mathrm{CS}^{+}\right)$gepaart wurden und ein weiterer Stimulus $\left(\mathrm{CS}^{-}\right)$ohne Distensionen präsentiert wurde. Nach der Akquisition erfolgte eine Extinktionsphase, in welcher alle CS ohne US präsentiert wurden. Zur Reaktivierung der Gedächtnisspur wurden im anschließenden Reinstatement zunächst unangekündigte Distensionen appliziert, nachfolgend erfolgte die alleinige Präsentation der visuellen CS. Gruppenunterschiede wurden auf Verhaltens- (VAS-Ratings der Valenz und Schmerzintensität) und BOLD-Ebene in Reaktion auf die Antizipation (CS) und Verarbeitung des Schmerzes (US) analysiert.

Auf neuronaler Ebene zeigten sich differentielle CS-induzierte BOLDAntworten in RDS-Patientinnen $\left(\mathrm{p}_{\mathrm{FWE}}<.05\right)$ im Vergleich zu gesunden Kontrollprobandinnen. Höhere Aktivierungen zeigten sich insbesondere im Kontrast $\mathrm{CS}^{-}>\mathrm{CS}^{+}$im Vermis, in der intermediären Zone und der posterolateralen Hemisphäre. In der Extinktion und im Reinstatement zeigten sich keine signifikanten Gruppenunterschiede.

Patientinnen mit Reizdarmsyndrom zeigen während der Furchtkonditionierung höhere neuronale Aktivierungen in medialen, intermediären und lateralen Bereichen des Kleinhirns. Diese Bereiche umfassen autonome, somatosensorische und kognitive Funktionen und tragen somit $\mathrm{zu}$ differentiellen Effekten der Schmerz-assoziierten Furcht bei. Neben dem Großhirn könnte somit das Kleinhirn einen maßgeblichen Anteil an der Entwicklung und Manifestation chronischer viszeraler Schmerzen haben.

\section{P04 - Experimentelle Schmerzmodelle (Tiermodelle)}

\section{P04.01 - Untersuchung Bewegungs-evozierten Schmerzverhaltens bei Ratten nach einer Inzision}

\section{Schäfer', D. Segelcke', M. Augustin', E. Pogatzki-Zahn'}

'Universitätsklinikum Münster, Klinik für Anästhesiologie, postoperative Intensivmedizin und Schmerztherapie, Münster, Deutschland

Ziel: Die Analyse von Schmerzverhalten in tierexperimentellen Studien beruht im Wesentlichen auf der Applikation verschiedener Stimuli wie Hitze oder von Frey Filamenten (vFF). Um Äquivalente zu mehr klinisch relevanten Schmerzphänomenen nach einer Verletzung/Operation zu evaluieren, haben wir hier ein Bewegungs-evoziertes Schmerzverhalten nach einer Inzision im plantaren Aspekt der Rattenpfote untersucht und mit der Erfassung der mechanischen Hyperalgesie mittels $v F F$ verglichen.

Methodik: Männliche Sprague Dawley Ratten ( $n=20,250-300$ g) erhielten eine standardisierte Inzision an der rechten Hinterpfote bzw. Sham-Inzision. Mittels kalibriertem $v F F(14,20,39,59,78,98,147,255,588 \mathrm{mN})$ wurde die mechanische Schmerzschwelle (PWT) vor und nach Inzision (bis POD 28, PostOperative Day) bestimmt. Für das Bewegungs-evozierte Schmerzverhalten wurde das CatWalk XT System (Version 10,5, Noldus) eingesetzt, welche eine Ganganalyse mit statischen (z. B. Kontaktfläche der Pfote) und dynamischen Parametern (z. B. Zeit, Geschwindigkeit) ermöglicht.

Ergebnisse: Die PWT nahm von $588 \mathrm{mN}$ (Median; vor Inzision) auf 59 $\mathrm{mN}$ (6 h nach Inzision) signifikant ab und war bis POD 3 reduziert. Die Ganganalyse (CatWalk-XT System) ergab eine Reduktion der Geschwindigkeit der Schwungphase der inzidierten Pfote von $94,5 \pm 12,54 \mathrm{~cm} / \mathrm{s}$ (Mittelwert \pm SD) vor auf $64,01 \pm 4,53 \mathrm{~cm} / \mathrm{s} 6 \mathrm{~h}$ nach Inzision, wonach diese graduell wieder anstieg (bis POD $14 p<0,05$ vs. vor Inzision und Sham). Analog dazu war die Schwungzeit der rechten Hinterpfote nach Inzision bis POD 14 signifikant verlängert ( $p<0,05$ vs. vor Inzision und Sham). Die Kontaktfläche der inzidierten Pfote verringerte sich signifikant von $0,25 \pm 0,12 \mathrm{~cm}^{2}$ vor auf $0,14 \pm 0,08 \mathrm{~cm}^{2} 6 \mathrm{~h}$ nach Inzision und stieg danach graduell wieder an ( $p<0,05$ bis POD 14 vs. vor Inzision und Sham). An POD 28 waren alle Gangparameter wieder vergleichbar zum Basiswert und zur Sham-Gruppe ( $p>0,05$ vs. vor Inzision und Sham). Im Vergleich dazu zeigte die Sham-Gruppe über den gesamten Beobachtungszeitraum keine signifikanten Verhaltensänderungen ( $p>0,05$ vs. vor Inzision).

Diskussion: Mit Hilfe des CatWalk XT Systems konnte eine zeitabhängige, graduelle Veränderung im Gangbild der Ratten nach einer Inzision gezeigt werden. Im Vergleich zur klassischen Messung der mechanischen Hyperalgesie mit vFF konnte ein klinisch relevantes Bewegungs-evoziertes Schmerzverhalten über einen längeren Untersuchungszeitraum nachgewiesen werden. Entweder unterscheiden sich die zugrunde liegenden Mechanismen des Bewegungs-evozierten Schmerzverhaltens von jenen der mechanischen Hyperalgesie und/oder ersteres ist sensitiver. Die Ganganalyse nach Inzisi- 
on könnte demnach die sensitivere und klinisch relevantere Methode zur Untersuchung von Schmerzverhalten nach Inzisionsverletzung darstellen.

\section{P04.02 - Die Rolle des GABA-Transporters GAT-1 und der Glutamatdecarboxylase GAD 65 und 67 für Hyperalgesie nach Inzisionsverletzung der Ratte}

\section{A. Cremer', M. Beyer', D. Segelcke', S. Reichl', E. Pogatzki-Zahn' \\ 'Universitätsklinikum Münster, Klinik für Anästhesiologie, operative Intensivmedizin und Schmerztherapie, Münster, Deutschland}

Einleitung: Die inhibitorische Funktion von GABA auf spinaler Ebene ist für verschiedene Schmerzentitäten nachgewiesen, genaue Mechanismen sind aber nicht bekannt. Ziel dieser Arbeit ist es, den Metabolismus von GABA über Synthese und uptake via GABA-Transporter nach Inzision im Tiermodell zeitabhängig zu untersuchen.

Methoden: Bei 44 männlichen SD-Ratten wurde unter Allgemeinanästhesie eine $1 \mathrm{~cm}$ lange Schnittinzision durch Haut, Faszie und Muskel des plantaren Aspekts der rechten Hinterpfote durchgeführt und mit einer chirurgischen Naht verschlossen. In in vivoVerhaltensuntersuchungen wurde vor und 2 Stunden nach Inzision die mechanische- (PWT) und thermale (HPT) Schmerzschwelle ermittelt, anschließend ein GABA-Transporter (GAT-1) Inhibitor (NO-711, 10, 20 und $40 \mu \mathrm{g}$, Vehikel 0,9\% Saline) intrathekal (IT) verabreicht $(n=5)$ und die Schmerzschwellen 15 bis 90 min nach Gabe erneut bestimmt. Mittels molekularbiologischer Methoden, wie Proteinexpressionsanalysen und immunhistochemischen Färbungen (IHC) zu verschiedenen Zeitpunkten (Prä, 4 h, 1d, 3d, 5d, 7d, $n=4$ pro Zeitpunkt) nach Inzision, wurde das Expressionsprofil für GAT-1 und die Glutamatdecarboxylase 65/67 (GAD 65/67) im lumbalen Rückenmark bestimmt. Ergebnisse: Der PWT nahm von $588 \mathrm{mN}$ (Median; vor Inzision) auf 58 $\mathrm{mN}$ ( $2 \mathrm{~h}$ nach Inzision) signifikant ab; $30 \mathrm{~min}$ nach GAT-Inhibitor $(40 \mu \mathrm{g})$ stieg der PWT signifikant auf $147 \mathrm{mN}$ an ( $p<0,05$ vs.Prä und Vehikel). Die niedrigeren Dosierungen hatten keinen Effekt. Die HPT (3,9 $\pm 0,57$, Mittelwert \pm SD, 2 h nach Inzision) konnte durch die IT Gabe von NO-711 nach 15 und $30 \mathrm{~min}(7,82 \mathrm{~s} \pm 1,11)$ signifikant angehoben werden $(p<0,05$ vs Prä und Vehikel). Die Expression von GAT-1 war 4 Stunden nach Inzision signifikant erhöht ( $151 \% \pm 24,71$ im Vergleich zur Sham-Inzision). Die Expression von GAD 67, jedoch nicht die von GAD 65, war zum gleichen Zeitpunkt signifikant reduziert $(54 \% \pm 7,45)$. Sowohl GAT- 1 , als auch GAD65/67 wurden mittels IHC in präsynaptischen Strukturen lokalisiert zusätzlich war die GAT-1 Färbung mit GFAP (Marker für Astrozyten) -positiven Zellen kolokalisiert.

Diskussion: Unsere Studie zeigt, dass GAT-1 eine Rolle in der Ausprägung mechanischer und thermaler Hyperalgesie nach Inzision spielt. GAT-1 wird vier Stunden nach Inzision in präsynaptischen Strukturen und GFAPpositiven Zellen überexprimiert; dies bedingt möglicherweise eine verstärkte Wiederaufnahme von GABA aus dem synaptischen Spalt, was eine reduzierte tonische GABAerge Wirkung an der Postsynapse und damit Reduktion der Schmerzschwellen (Hyperalgesie) bedingen könnte. Die Reduktion von GAD 67 nach Inzision kann möglicherweise dadurch erklärt werden, dass die vermehrte Aufnahme von GABA in präsynaptische Strukturen (durch vermehrte GAT-1-Expression) die Notwendigkeit der Neusynthese von GABA aus Glutamat vermindert. Humane und klinische Studien müssen zeigen, ob GAT-1 Antagonisten zur Verminderung von postoperativen Schmerzen und Hyperalgesie eingesetzt werden können.

\section{P04.03 - Einfluss niedrigdosierter postnataler Bestrahlung auf die zentralnervöse Prozessierung nozizeptiver Hitzereize bei Mäusen}

\section{S. Kreitz' ${ }^{1}$ S. Kreitz ${ }^{2}$, M. Uder ${ }^{2}$, A. Hess' ${ }^{1}$ S. Strobelt ${ }^{1}$}

'Institut für Experimentelle und Klinische Pharmakologie und Toxikologie, Friedrich-Alexander-Universität Erlangen-Nürnberg, Pharmakologische Bildgebung, Erlangen, Deutschland, ${ }^{2}$ Universitätsklinikum Erlangen, Radiologische Abteilung, Erlangen, Deutschland

Die Verarbeitung von Schmerz ist ein komplexer und vielschichtiger Prozess, der auch höhere assoziative Zentren des Gehirns involviert. Stö- rungen in der Entwicklung des Gehirns könnten sich also auf die zentralnervöse Prozessierung von Schmerz auswirken - unabhängig vom subjektiven Schmerzempfinden.

In der vorliegenden Arbeit wurden Mäuse 10 Tage nach der Geburt mit 500 mGray bestrahlt (Ganzkörper). Die adulten Tiere wurden dann im BOLD-fMRT mit nicht nozizeptiven $\left(40^{\circ} \mathrm{C}\right.$ und $\left.45^{\circ} \mathrm{C}\right)$ und nozizeptiven $\left(50^{\circ} \mathrm{C}\right.$ und $\left.55^{\circ} \mathrm{C}\right)$ Hitzereizen an der rechten Hinterpfote stimuliert. Anschließend wurde die mittels einer GLM determinierte stimulusspezifische Gehirnaktivierung sowie die Konnektivität zwischen den aktivierten Regionen mittels Graphtheorie im Vergleich zu einer Kontrollgruppe ohne Bestrahlung untersucht. Zusätzlich wurden die Tiere eine „Resting-state“Messung ohne Stimulation unterzogen.

Im Vergleich zu der Kontrollgruppe zeigten die bestrahlten Tiere ein deutlich verändertes „Resting-state“-Netzwerk, welches durch disruptive Konnektivitäten vor allem im Bereich des „Default-Mode“-Netzwerkes charakterisiert ist. Die „Resting-state“-Netzwerke der bestrahlten Tiere weisen einen niedrigeren „Small-World-Index“ auf als die der Kontrolltiere. Der "Small-World-Index" ist ein graphtheoretischer Parameter, der die Effizienz des Informationsflusses eines Netzwerkes widerspiegelt.

Die stimulusspezifische Gehirnaktivierung war bei den bestrahlten Tieren generell in den subkortikalen Gehirnregionen stärker als bei den Kontrolltieren. Eine Reduktion der Aktivierung war bei nozizeptiver Stimulation im Kortex und bei nicht nozizeptiver Stimulation im Hippocampus zu beobachten. Durch die graphtheoretische Analyse der Konnektivitäten zwischen den aktivierten Hirnregionen konnten zusätzliche Einsichten in diesen Effekt gewonnen werden. Auch hier findet die Reduktion der Verbindungen bei den bestrahlten Tieren vor allem zwischen kortikalen Strukturen und die Verstärkung hauptsächlich von subkortikalen Strukturverbindungen statt: bei nozizeptiver Stimulation zeigt vor allem der Thalamus verstärkte Verbindungen, während bei der nicht nozizeptiven Stimulation Basalganglien, Colliculi, Amygdala und piriformer Kortex (als Verbindung zum limbischen System) vermehrte Konnektivitäten aufweisen. Die Reduktion der Verbindungen betrifft im Falle der nozizeptiven Stimulation ausschließlich den Kortex während bei der nicht nozizeptiven Stimulation auch der Hippocampus stark betroffen ist.

Zusammenfassend zeigte sich, dass die postnatale Bestrahlung mit 500 mGray zu einer Beeinträchtigung der Gehirnentwicklung führt, die sich nicht nur in einem disruptiven „Resting-State“ Netzwerk mit beeinträchtigtem Informationsfluß sondern auch in einer Veränderung der zentralnervösen Schmerzprozessierung manifestiert. In letzterer sind vor allem die höher entwickelten Gehirnstrukturen des Kortex betroffen, deren Entwicklung auch postnatal noch stattfindet und deshalb stärker von der Bestrahlung beeinflusst wird.

\section{P04.04 - BOLD-Signal-basierte graphtheoretische Untersuchung der differentiellen Effekte verschiedener Anästhetika und Stimulationsparadigmen auf das laterale und mediale Schmerzsystem in Ratten}

T. Makarova' ${ }^{1}$ M. Sergeeva' ${ }^{1}$, A. Hess ${ }^{2}$

'FAU Erlangen-Nuremberg, Dep. of Experimental and Clinical Pharmacology, Erlangen, Deutschland, ${ }^{2}$. f. Experimentelle Pharmakologie, Pharmakologische Bildgebung/Bildanalyse, Erlangen, Deutschland

Das zentralnervöse Schmerzverarbeitungssystem kann in einen lateralen und medialen Anteil unterteilt werden (Treede et al., 1999 Feb, Pain, The cortical representetion of pain). Strukturen des lateralen Schmerzsystems (LS: lateraler Thalamus, somatosensorische Kortizes (cxSS)) sind für die sensorisch-diskriminative Komponente des Schmerzes (Lokalisation und Intensitätsbestimmumg der Schmerzereignisse) verantwortlich, Strukturen des medialen Systems (MS: medialer Thalamus, Assoziationskortizes (cxAss)), die mit dem limbischen System eng verbunden sind, für die affektiv-emotionale Komponente (affektive Beurteilung der Schmerzreize). Unterschiedliche Reize können in unterschiedlichem Maße durch das LS oder MS verarbeitet werden. Aber auch verschiedene Substanzen können die 2 Systeme unterschiedlich modulieren. In dieser Studie wurden die Auswirkungen von 3 Anästhetika (Isofluran (ISO), Medetomidin (MED) 
und Propofol (PRO)) auf das Schmerzverarbeitungssystem unter Berücksichtigung 2 unterschiedlicher Schmerzparadigmen $\left(55^{\circ} \mathrm{C}\right.$-Hitze- und Elektro-Stimulation, Hinterpfote der Ratte) mittels BOLD-fMRT erfasst und mittels Graphtheorie analysiert. Die Elektro-Stimulation sollte durch ihre zusätzliche unangenehme Komponente größere Einflüsse auf das MS haben. ISO ist hypnotisch und (schwach) analgetisch wirksam, MED ist sedierend und stark analgetisch (nur unüblich hoch dosiert hypnotisch) und PRO ist fast nur hypnotisch.

Mittels klassischem BOLD-fMRT ist uns bereits gelungen die Hypothese aufzustellen, dass die hypnotische Wirkung vorwiegend über die affektivemotionale und die analgetische über die sensorisch-diskriminative Komponente zustande kommt (Poster Makarova 2015)

Basierend auf einer kompletten Kreuz-Korrelationsmatrix der Zeitverläufe aller aktivierten Gehirnstrukturen wurden graphtheoretische/Community Analysen durchgeführt und die funktionelle Konnektivität zwischen dem LS und MS näher untersucht.

Unter ISO ergaben sich keine Unterschiede zwischen der $55^{\circ} \mathrm{C}$ - und der vergleichbar schmerzhaften Elektro-Stimulation: am stärkste korrelierten der Thalamus (th) mit dem Hippocampus (hc) und cxAss mit cxSS.

Unter MED war bei $55^{\circ} \mathrm{C}$ ein ähnliches Bild zu sehen. Dagegen waren bei der Elektro-Stimulation die intra- und extra-thalamischen Verbindungen gestärkt (vor allem zum hc und cxAss). Durch die zusätzliche unangenehme Komponente scheint die Elektro-Stimulation die funktionelle Konnektivität zwischen th und cxAss zu verstärken. Die Verbindungen zwischen cxAss und cxSS, die jeweils eine eigene Community bildeten, waren deutlich geschwächt, vermutlich durch die starke Analgesie.

Die PRO-Anästhesie führte allgemein zu einem diffuseren Netzwerk mit schwächeren intrakortikalen Verbindungen, wobei bei $55^{\circ} \mathrm{C}$ nur geringe Unterschiede zu ISO und MED beobachtet wurden. Bei der ElektroStimulation ging die enge Vernetzung zwischen th und cxAss verloren (s. MED), vermutlich durch die ausgeprägten hypnotischen Eigenschaften von PRO. Gemeinsam mit den fMRT-Ergebnissen bestätigen diese Ergebnisse unsere Hypothese.

\section{Literatur}

1. Treede RD, Kenshalo DR, Gracely RH, Jones AK (1999) The cortical representation of pain. Pain 79:105-111

2. Differentielle Effekte verschiedener Anästhetika und Stimulationsparadigmen auf das laterale und mediale Schmerzsystem in Ratten: funktionelle MRT (fMRT) Studie; Poster P04.07, Makarova, Schmerzkongress 2015

\section{P04.05 - Zerebrales Korrelat der spinalen pharmakologischen Modulation des GABAA Rezeptors in einem Tiermodell für postoperative Schmerzen}

\section{Segelcke', N. Just ${ }^{2}$, C. Faber' ${ }^{2}$, E. Pogatzki-Zahn'}

'Universitätsklinikum Münster, Klinik für Anästhesiologie, postoperative Intensivmedizin und Schmerztherapie, Münster, Deutschland, ${ }^{2}$ Institute for Clinical Radiology, Translational Research Imaging Center (TRIC), Münster, Deutschland

Ziel: Die Methode der funktionale Magnet Resonanz Tomographie (fMRT) wird in den letzten Jahren vermehrt in der Schmerzforschung eingesetzt, um zerebrale Korrelate und Netzwerke der Schmerzverarbeitung in verschiedenen Tiermodellen zu ermitteln. In einer vorherigen Studie konnte der Effekt einer in vivo Modulation von spinalen $\mathrm{GABA}_{\mathrm{A}}$-Rezeptoren in einem Inzisions-Model auf die mechanische Hyperalgesie gezeigt werden [1]. Das Ziel dieser Studie ist es, die zerebralen Korrelate dieser pharmakologischen Modulation nach einer überschwelligen mechanischen Stimulation im Inzisions-Model zu detektieren.

Methoden: Sprague Dawley Ratten ( $n=15,180-250$ g, ठै) erhielten einen intrathekalen (IT) Katheter und drei Tage später eine standardisierte Inzision an der rechten Hinterpfote, welche $24 \mathrm{~h}$ vor der MRT-Messung (Medetomidin-Narkose) in einem 9,4T Bruker Biospec mit einer EPI Sequenz (TR/TE: $1000 / 18 \mathrm{~ms}, 1,2 \mathrm{~mm}$ thick, FOV $30^{*} 30 \mathrm{~mm} 2$, Matrix $80^{\star} 80$, 600 averages) durchgeführt wurde. Die mechanische Stimulation (95 gr.) wurde auf dem plantaren Aspekt der ipsilateralen Hinterpfote in einem Block-Design (10s Stimulation, 20s Pause) für 20 Zyklen appliziert. Es erfolgte eine Messung 0 min und 60 min nach IT-Gabe eines $\mathrm{GABA}_{\mathrm{A}^{-}}$ Rezeptor-Agonisten (Muscimol, 0,3 $\mu \mathrm{g}, \mathrm{Mu}$ ) oder -Antagonisten (Bicuculline, $2 \mu \mathrm{g}$, Bi), oder Vehikel (0,9\% Saline, V). Die Gruppen-Analyse erfolgte durch SPM.

Ergebnisse: Zum Zeitpunkt 0 min konnten zwischen den Gruppen keine Unterschiede hinsichtlich der Anzahl der aktivierten oder deaktivierten Voxel festgestellt werden (U-test, $p>0,05$ ). Nach 60 min reduzierte sich die Anzahl der aktivierten Voxel in der Mu-Gruppe signifikant von $553 \pm 355$ (Mittelwert \pm SD) auf $235 \pm 114(p<0,05)$ während der mechanischen Stimulation. In der Bi-Gruppe konnte ein tendenzieller Anstieg (306 \pm 269 nach $0 \mathrm{~min}$, auf $1114 \pm 1278$ nach $60 \mathrm{~min}$ ) der aktvierten Voxel gezeigt werden. Zusätzlich sollen spezifische Areale identifiziert werden, die nach spinaler GABAerger Modulation in die Reduktion von Hyperalgesie nach Inzision involviert sind.

Diskussion: Nach IT-Applikation eines $\mathrm{GABA}_{\mathrm{A}}$-Agonisten konnte eine signifikante globale zerebrale Deaktivierung detektiert werden, wohin gegen die Inhibition des Rezeptors tendenziell eine erhöhte Anzahl aktivierter Voxel nach mechanischer Stimulation zur Folge hatte. Aus in vivo Verhaltensuntersuchungen im Inzisions-Model ist bekannt, dass die Aktivierung des $\mathrm{GABA}_{\mathrm{A}}$-Rezeptors zu einer verminderten mechanischen Hypersensitivität führt, und dass die Inhibition eine gesteigerten Hyperalgesie erzeugt [1]. Spontanes (nicht-evoziertes) Schmerzverhalten wird durch spinale GABAerge Modulation nicht beeinflusst [1]. Die MRT-Methodik gepaart mit einer spinalen pharmakologischen Modulation (phMRT) des $\mathrm{GABA}_{\mathrm{A}}$-Rezeptors kann damit wichtige Informationen über die zerebrale Verarbeitung von mechanischer Hyperalgesie nach Inzision liefern.

\section{Literatur}

1. Reichl S, Augustin M, Zahn PK (2012) Pogatzki-Zahn EM. Pain153:129-141

\section{P04.06 - Geschlechtsspezifische Unterschiede beim Schmerz in Osteoarthrose-Mäusen}

J. Temp' ', M. Pannell', D. Labuz', H. Machelska'

${ }^{1}$ Charité- Universitätsmedizin Berlin, Campus Benjamin Franklin, Klinik für Anästhesiologie m.S. operative Intensivmedizin, Berlin, Deutschland

Einleitung: Schmerz ist eines der wichtigsten Symptome der Osteoarthrose (OA) und kommt durch eine verstärkte Innervation und Aktivierung von peripheren und zentralen sensorischen Neuronen durch entzündungsfördernde und Schmerz verstärkende Mediatoren, die aus den betroffenen Gelenken und aus Neuronen im zentralen Nervensystem freigesetzt werden, zustande. Da geschlechtsspezifische Unterschiede in der Prävalenz der OA vorliegen, mit erhöhter Prävalenz für für Frauen, war das Ziel dieser Studie geschlechtsspezifische Unterschiede beim Schmerz in Mäusen mit chirurgisch induzierter OA zu untersuchen.

Methoden: Die Verhaltensversuche wurden an je 12 C57BL76j Mäusen pro Geschlecht und pro Gruppe unter Einhaltung der ARRIVE Richtlinien und mit Genehmigung der LAGeSO durchgeführt. OA wurde in den rechten Kniegelenken durch eine Transsektion des medialen Kollateralbandes sowie des medialen Meniskus induziert. Als Kontrollen dienten Schein- operierte Mäuse, ohne Transsektion des medialen Kollateralbandes und des medialen Meniskus, sowie naive Mäuse. Die thermische und mechanische Schmerzschwelle der Tiere wurde mittels Hargreaves und Von Frey Test über 3 Monate erhoben, ebenso die Lokomotoraktivität in open field Boxen und die dynamische Pfotenbelastung im dynamic weight bearing Gerät.

Ergebnisse: Männliche und weibliche OA-Mäuse zeigen postoperativ; die Weibchen jedoch 4 Tage später; eine Hitzesensibilisierung, welche nach 2-3 Tagen verschwindet. Eine vergleichbare Hitzesensibilisierung zeigt auch weibliche Schein-operierte Tiere, nicht jedoch bei den Männchen. Beide Geschlechter zeigen nach der OA-Induktion eine mechanische Hypersensibilisierung, die bei den Männchen postoperative nach 7 Tagen verschwunden ist und in einer zweiten Phase erneut chronisch auftritt (4 Wochen - 3 Monate). Die OA-Weibchen hingegen zeigen keinen zweiphasischen Verlauf; die erniedrigte Schmerzschwelle verbleibt den ganzen Untersuchungzeitraum von 3 Monaten. Schein-operierten Tieren zeigen nur postoperative eine Hypersensibilisierung, wobei die der Weibchen stärker 
ist. Trotz der mechanischen Hypersensibilisierung zeigen OA-Mäuse, geschlechtsunspezifisch, keine reduzierte Aktivität. Interessanterweise aber zeigen die OA Mäuse eine geringere Belastung der operierten Pfote (ebenfalls zweiphasisch).

Schlussfolgerung: Das klinisch relevante InnenmeniskustranssektionsMausmodell der OA spiegelt die Beschwerden, hauptsächlich mechanische Schmerzen und Fehlbelastung des Knies, der OA Patienten wieder. Außerdem scheinen geschlechtsspezifische Unterschiede, mit einer stärkeren und länger andauernden mechanischen Hypersensibilisierung der weiblichen Tiere vorzuliegen. Eine getrennte Betrachtung von männlichen und weiblichen Mäusen in tierexperimentellen Osteoarthrose Studien sollte dementsprechend immer vorgenommen werden.

\section{P04.07 - Zentralnervöse Effekte der Ablation von TRPV1- exprimierenden nozizeptiven Neuronen via Resiniferatoxin auf die Temperaturprozessierung}

\author{
I. Wank', L. Kutsche', S. Kreitz', A. Hess ${ }^{1}$ \\ 'Institut für Pharmakologie und Toxikologie, FAU Erlangen, Bildgebung, \\ Erlangen, Deutschland
}

Resiniferatoxin (RTX) ist ein natürlich vorkommendes Diterpen, welches im Saft verschiedener Wolfsmilchgewächse der Gattung Euphorbia enthalten ist. 100-fach potenter als Capsaicin, wirkt es analog als Aktivator des TRPV1-Kanals, einem unselektiven Kationenkanal mit einer hohen Permeabilität für Calcium-Ionen. Dieser wird im peripheren Nervensystem vorwiegend auf den Nervenendigungen von C-Fasern exprimiert, wo er wesentlich für deren Nozizeptorfunktion ist. TRPV1-Kanäle werden durch verschiedene endogene Liganden (viele Entzündungsmediatoren) sowie Temperaturen über $43^{\circ} \mathrm{C}$ aktiviert. Da RTX in hohen Dosen zytotoxisch wirkt, kann es dazu eingesetzt werden selektiv TRPV1-exprimierende Neuronen zu ablatieren. Dieser Effekt kann je nach Applikationsart einzelne Nervenstränge, Dermatome oder den ganzen Körper betreffen. Ziel der vorgestellten fMRT-Studie war die Untersuchung der zentralen Temperaturprozessierung der Ratte, mit und ohne nozizeptiven Input aus der Körperperipherie. Während einer 100minütigen BOLD-fMRT-Messung wurde eine pseudorandomisierte Hitzereizsequenz (8 Temperaturen zwischen $40^{\circ} \mathrm{C}$ und $54^{\circ} \mathrm{C}, 2 \mathrm{Grad}$ Unterschied, linke Hinterpfote) präsentiert. Nach einer Basis-BOLD-fMRT-Messung wurde den Tieren an 3 aufeinander folgenden Tagen in steigender Dosis $30 \mu \mathrm{g}, 70 \mu \mathrm{g}$ und $100 \mu \mathrm{g}$ RTX, aufgeteilt auf zwei s. c.-Injektionen pro Tag, verabreicht. Nach 8 weitere Tagen erfolgte die post-RTX MRT-Messung.

Anhand klassischer BOLD-Analyse sowie modernen graphtheoretischen Methoden konnten wir zeigen, dass RTX effektiv nozizeptiven Input aus der Körperperipherie blockiert. Die Behandlung ist kanalspezifisch, da wir unter $48^{\circ} \mathrm{C}$ (mediiert durch andere TRP-Kanäle) kaum Unterschiede in der Gehirnaktivität beider Gruppen finden. Im Vergleich zu den unbehandelten Tieren finden wir bei der postRTX-Gruppe ab $48^{\circ} \mathrm{C}$ (nozizeptiv) eine großräumige Abnahme der stimulus-induzierten Gehirnaktivität in fast allen Bereichen mit Ausnahme des Hirnstammes.

Netzwerkanalysen ergaben nur geringe Unterschiede die Strukturen des Hirnstammes betreffend, ein wichtiger Hinweis dafür, dass der propriozeptive und sensorische Eingang von Pons, Medulla oblongata und dem lemniskalen System weitestgehend intakt scheint. Hingegen wird die nozizeptiv getriggerte Aktivität des Informationsverteilers Thalamus drastisch reduziert, was sich auch in einer Abnahme von intra- und extrathalamischen Verbindungen wiederspiegelt. Dennoch zeigen sich kortikale Verbindungen der behandelten Tiere eher gestärkt: kortikale Oszillationen laufen ohne peripheren Input ab und könnten daher durch die Ablation nicht reduziert, möglicherweise sogar verstärkt werden.

Mittels fMRT konnten wir zeigen, dass sich eine kanalspezifische Ablation nozizeptiver C-Fasern nicht im Hirnstamm manifestiert sondern sich vor allem durch reduzierten thalamischen Input auf upstream-organisierte Gehirnregionen auswirkt.

\section{P04.08 - Molekulare und funktionelle Veränderungen im nozizeptiven System der Ratte beim Diabetes mellitus - Beteiligung von TRP-Kanälen?}

\section{B. Schlickenrieder', U. Binzen' ', R. Treede', W. Greffrath ${ }^{3}$}

'Medizinische Fakultät Mannheim der Universität Heidelberg, Lehrstuhl für Neurophysiologie, Zentrum für Biomedizin und Medizintechnik Mannheim (CBTM), Mannheim, Deutschland, ${ }^{2}$ Universität Heidelberg, Lehrstuhl für Neuropyhsiologie, Mannheim, Deutschland, ${ }^{3}$ Medizinische Fakultät Mannheim der Universität Heidelberg, Mannheim, Lehrstuhl für Neurophysiologie, Zentrum für Biomedizin und Medizintechnik Mannheim, Mannheim, Deutschland

Einleitung: Die schmerzhafte Polyneuropathie ist eine häufige Komplikation des Diabetes mellitus. Sie zeichnet sich durch ein gemischtes Nebeneinander von sensorischen Plus- und Minussymptomatiken aus, wobei neben Sensibilitätsverlusten Hyperalgesie und Allodynie beobachtet werden kann, die häufig zu einem schwer behandelbaren chronischen Schmerzsyndrom führen. Im Rahmen dieser Studie wurden molekulare Veränderungen im nozizeptiven System der Ratte untersucht, die dieser Symptomatik zu Grunde liegen könnten.

Methoden: Mittels Injektion von Streptozotocin wurde in der Ratte ein Diabetes mellitus vom Typ 1 (T1 DM) induziert, zur Untersuchung des T2 DM diente ein etabliertes Tiermodell, die Zucker Diabetic Fatty (ZDF-) Ratte, Zucker Fatty (ZF-)Ratten dienten der Untersuchung metabolischer Einflüsse ohne diabetische Hyperglykämie. In den Spinalganglien dieser diabetischen Tiere wurden Veränderungen der Genexpression bzw. der Proteinexpression nozizeptiver Membrankanäle gegenüber Kontrolltieren mittels Microarray-Analysen (Affymetrix Rat Gene 2.0 ST Array GeneChips) und Immunohistochemie analysiert. Der Verlauf der Hitzesensitivität gegen schmerzhafte Nahinfrarot-Laserstimulation wurde über den Verlauf der Entwicklung des T1 DM charakterisiert.

Ergebnisse: Unter diabetischem Einfluss zeigte die Genexpression eine Reduktion unseres primären Zielgenes, des nozizeptiven Transduktionsmoleküles TRPV1, um 16\% $(p=0,05)$ beim T1 DM und eine leichte Steigerung von TRPV1 bei den ZF-Ratten (22\%). Nur bei T1 DM fand sich eine Hochregulation des Purinorezeptors P2X3. Die Expression TRPV1 war jedoch auf Proteinebene in keinem der untersuchten Modelle verändert, wohingegen P2X3 beim T1 DM und in den ZF Tieren signifikant häufiger exprimiert wurde. Im Rahmen des T1 DM fand sich nach 8 Wochen eine Schwellenabsenkung für schmerzhafte Laserhitzereize auf ca. $70 \%$ des Ausgangswertes im Sinne einer Hyperalgesie $(71,7 \pm 6,06 \%$ versus $91,8 \pm 6,32 \%$ in Kontrollen; $p<0,01$ LSD Test).

Diskussion: Der Capsaicin-Rezeptor TRPV1 scheint beim Diabetes mellitus keine relevant geänderte Expression zu zeigen, zumindest nicht innerhalb von 8 Wochen nach bestehendem T1 DM. Die beobachtete diabetische Hyperalgesie gegen Hitzereize kann dabei indirekt durch gesteigerte Expression des Puriniorezeptors P2X3 erklärt werden und/oder durch Sensibilisierung am TRPV1. Laserhitzereiz-Untersuchungen erscheinen daher zur Charakterisierung einer schmerzhaften diabetischen Polyneuropathie im Tiermodell als sinnvoll.

\section{P04.09 - Immunohistochemical study of spinal glial cell activation in an animal model of non-specific low back pain}

J. Zhang', U. Hoheisel', S. Mense ${ }^{3}$, R. Treede ${ }^{4}$

${ }^{1}$ Medizinische Fakultät Mannheim der Universität Heidelberg, Mannheim, Lehrstuhl für Neurophysiologie, Zentrum für Biomedizin und Medizintechnik Mannheim, Mannheim, Germany, ${ }^{2}$ Zentrum für Biomedizin und Medizintechnik, Universitätsmedizin Mannheim, Lehrstuhl für Neurophysiologie, Mannheim, Germany, ${ }^{3}$ Universität Heidelberg, Mediz. Fakultät Mannheim, CBTM, Neuroanatomie, Mannheim, Germany, ${ }^{4}$ Universität Heidelberg, Lehrstuhl für Neuropyhsiologie, Mannheim, Germany

Background and aims: Several studies have showed that activated spinal glial cells play an important role in the development of chronic pain. Activated glial cells show a transformation from a ramified non-activated to an 
unramified activated morphological state. However, the role of glial cell activation in the development of low back pain is unclear. The present study investigated morphological changes of spinal glial cells in an animal model of non-specific low back pain using immunohistochemistry.

Methods: As an animal model of low back pain, two injections of nerve growth factor (NGF) were made into the rat multifidus muscle at an interval of 5 days. Vehicle injection served as a control. One day after the second injection, cross sections of segment L2 in the spinal cord were processed immunohistochemically for ionized calcium-binding adapter molecule 1 (Iba-1) and for glial fibrillary acidic protein (GFAP). Iba-1 and GFAP are specific marker molecules of microglia and astrocytes, respectively. Electrophysiological experiments showed that segment $\mathrm{L} 2$ receive strong afferent input from low back tissues. Digitized images of immunoreactive glial cells were obtained with a laser-scanning microscope. To evaluate morphological changes indicating glial cell activation two methods were used: 1) analysis of the whole Iba-1 or GFAP immunoreactive area (size, perimeter, Feret's diameter, circularity and solidity of immunoreactive areas). 2) Sholl analysis of single glial cells.

Results: The immunohistochemical results showed obvious morphological changes of microglial cells 1 day after the second NGF injection. All results indicated a transition from a ramified non-activated form to an unramified activated form in the entire dorsal horn after the two NGF injections. Compared to the control, the analysis of the whole Iba- 1 immunoreactive area indicated that after NGF injections microglial cells exhibited a larger soma size with a smaller perimeter, resulting in a smaller Feret's diameter, a larger circularity and a larger solidity (all $\mathrm{P}<0,05)$. The Sholl analysis of single cells confirmed this data. Single microglial cells had fewer ramifications and shorter processes after NGF injections. In contrast, the morphological changes of astrocytes in the spinal dorsal horn were not obvious 1 day after the second NGF injection, indicating that astrocyte activation was only weak. Conclusions: The immunohistochemical data showed prominent morphological changes of microglial cells 1 day after the second NGF injection in the entire spinal dorsal horn, indicating strong microglial activation in the animal model of non-specific low back pain used in the present study. In contrast to microglial cells, in astrocytes the immunohisochemical signs of activation were only weak.

Acknowlegements: We acknowledge the support of the Core Facility Live Cell Imaging Mannheim at the CBTM funded by DFG (INST 91027/9-1 FUGG).

\section{P05 - Kopfschmerz/Pflege/Aus- und Weiterbildung}

\section{P05.01 - Repetitive Magnetstimulation der Nackenmuskulatur (m. trapezius) bei Patienten mit Migräne}

\section{N. Sollmann' ', F. Trepte-Freisleder' , F. Heinen², S. Krieg', M. Landgraf ${ }^{2}$}

'Klinikum rechts der Isar, Technische Universität, Neurochirurgische Klinik, München, Deutschland, ${ }^{2}$ Kinderklinik im Haunerschen Kinderspital, LudwigMaximilans-Universität, Neuropädiatrie, München, Deutschland

Hintergrund: Die repetitive periphere Magnetstimulation (rPMS) hat wiederholt positive Effekte bei der Behandlung myofaszialer Schmerzen gezeigt. Migräne steht, laut aktueller Studienlage, mit myofaszialen Schmerzen der Nacken-Schulter-Region in Verbindung (entsprechend dem Konzept des „trigemino-cervical complex“ (TCC)). In dieser Studie wird die Umsetzbarkeit und Akzeptanz einer rPMS-Behandlung des oberen Musculus trapezius bei Menschen mit Migräne untersucht.

Methode: 20 Probanden mit Migräne wurden in die Studie eingeschlossen. Aktive myofasziale Triggerpunkte (TrPs) des oberen Trapeziusmuskels wurden mit rPMS stimuliert. Die Akzeptanz wurde anhand eines standardisierten Fragebogens bewertet. Der potentielle Effekt der rPMS Intervention wurde per Kopfschmerzkalender und dem „Migraine Disability Assessment (MIDAS)“ Fragebogen beurteilt. Um eine mögliche lokale Auswirkung auf die Muskulatur zu erfassen, wurden per Algometrie die Druck-Schmerz-Schwellenwerte bestimmt.
Ergebnisse: Es sind keinerlei unerwünschte Nebenwirkungen aufgetreten - weder während, noch im Anschluss an die rPMS Behandlung. Vielmehr zeigte die rPMS eine signifikante Wirkung im Bezug auf eine Reduktion der Migräneintensität $(p=0,001)$ und der Anzahl von Migräneattacken $(p<0,001)$. Gemäß des standardisierten Fragebogens gaben 100,0\% der Patienten an, die Behandlung nochmals durchführen zu wollen und 90,0\% würden die rPMS als Therapieoption für Migräne empfehlen.

Schlussfolgerung: Die rPMS könnte in Form einer Behandlung myofaszialer Schmerzen eine vielversprechende Therapieoption für Menschen mit Migräne darstellen. Dies erklärt sich möglicherweise anhand der stimulationsbedingten Modulation des peripher-sensorischen Inputs innerhalb des TCCs bei Migräne. Um einen möglichen positiven Effekt zu beweisen, bedarf es größerer, placebo-kontrollierter Studien. Zukünftige Studien sollten zudem auch Kinder und Jugendliche mit Migräne einbeziehen.

\section{P05.02 - DreKiP - ein ambulantes multimodales Therapieprogramm für Kopfschmerzkinder}

E. Gruhl', A. Hähner', E. Lautenschläger', T. Müller', F. Schumann', D. Skiera', A. Theisinger', U. Zimmer', R. Berner ${ }^{3}$, M. von der Hagen ${ }^{4}$, R. Sabatowski ${ }^{5}$, M. Richter', G. Goßrau'

'Interdisziplinäre Kinderkopfschmerzambulanz am Universitätsschmerzcentrum Dresden, Dresden, Deutschland, Interdisziplinäres Riechzentrum, Universitätsklinik Dresden, Dresden, Deutschland, ${ }^{3}$ Klinik und Poliklinik für Kinder- und Jugendmedizin, Universitätsklinikum, Dresden, Deutschland, ${ }^{4}$ Uniklinikum Dresden, Abteilung Neuropädiatrie, Dresden, Deutschland, "UniversitätsSchmerzCentrum, Universitätsklinikum „Carl Gustav Carus", Dresden, Deutschland

Fragestellung: Kopfschmerzen sind ein häufiges Gesundheitsproblem bei Kindern und Jugendlichen. Biopsychosoziale Interaktionen modulieren das Auftreten von Kopfschmerzen im Kindesalter wesentlich. Ein ambulantes multimodales Programm für Kinder/Jugendliche mit regelmäßig auftretenden Kopfschmerzen soll kopfschmerzbedingte Einschränkungen im Alltag vermindern.

Methode: DreKiP = Dresdner KinderkopfschmerzProgramm ist ein multimodales Therapiegrogramm, bestehend aus 8 Modulen für Kinder/ Jugendliche: Edukation, Streßbewältigung, Entspannungsmethoden, körperliche Fitness, Klettertherapie, Kunsttherapie, Ergotherapie, sensorisches Training sowie 4 Modulen mit parallelen Eltern-Workshops. Es wird über 2-3 Monate ambulant, schulbegleitend durchgeführt. Die Gruppen bestehen aus 6-8 Patienten eines Alters. Insgesamt erhalten die Patienten $15 \mathrm{~h}$, die Eltern $7 \mathrm{~h}$ Therapie. Am Ende jedes Therapiemoduls wird ein gemeinsames Bewegungsspiel: Migräne schlägt Aktivität schlägt Ruhe schlägt Migräne usw. umgesetzt.

Begleitend zum Programm erfolgt die Dokumentation kopfschmerzspezifischer Daten, u. a. Häufigkeit, Medikamentengebrauch, Schulausfall. Ergebnisse: Bisher absolvierten 14 Jugendliche das DreKiP, eine Gruppe 14/15- und eine Gruppe 16/17-Jähriger. 9/14 Patienten mit Migräne und Spannungskopfschmerz, 3/14 mit Migräne und 2/14 mit chronischem Spannungskopfschmerz. Alle Patienten zeigen primäre Kopfschmerzen. 3/12 Migränepatienten präsentieren Migräne mit klassischer Aura. Die Kopfschmerztage pro Monat sind im Median 15 Tage, die offiziellen Schulfehltage im Median 3,5 Tage/Monat. Hier ist von nichterfassten Schulfehlzeiten auszugehen.

Die direkten Therapieergebnisse äußerten sich in verbesserter Akutbehandlung, besserer Wahrnehmung und Einordnung der Kopfschmerzen, körperlicher Aktivierung, Beginn von Entspannungsverfahren, Verbesserung im häuslichen und schulischem Umfeld. Der Effekt auf die Kopfschmerzhäufigkeit wird im Verlauf dokumentiert.

Schlussfolgerung: Das schulbegleitende multimodale Therapiegrogramm DreKiP verbessert Kopfschmerzedukation und das Umsetzen therapeutischer Möglichkeiten. Zielgruppe sind Kopfschmerzkinder/Jugendliche mit kopfschmerzbedingten Einschränkungen des Alltagslebens in Schulund Freizeit. 
P05.03 - Gesteigerte Schmerzempfindung am Kopf vor der Kopfschmerzattacke bei Migränepatienten: eine Möglichkeit zur zeitlichen Prognose von Migräneattacken?

\section{Strupf', K. Meßlinger', R. Sittl' ${ }^{2}$, B. Fraunberger ${ }^{2}$, B. Namer ${ }^{1}$ \\ 'Institut für Physiologie und Pathophysiologie, Universität Erlangen, Erlangen, Deutschland, 'Universitätsklinik Erlangen, Schmerzzentrum, Erlangen, Deutschland}

Bei Migränepatienten tragen - im Gegensatz zu Patienten mit Spannungskopfschmerz - wahrscheinlich cyclische Veränderungen der neuronalen Erregbarkeit zu dem typischen Muster von Schmerzattacken und schmerzfreiem Intervall bei. Neurone, deren Aktivität die Kopfschmerzintensität bestimmt, erhalten konvergente Zuflüsse von Afferenzen parikranialer Gewebe und der Dura mater encephali, welche über axonale Kollateralen ebenfalls extrakraniale Strukturen wie Periost und Muskelfaszien innervieren können. Deshalb können cyclische Veränderungen der Erregbarkeit auch außerhalb des Kopfes getestet werden.

Wir untersuchten die Schmerzschwellen und überschwelliges rating von mechanischen und elektrischen Reizen bei Patienten mit Migräne, Spannungskopfschmerz und gesunden Probanden am Kopf beidseits frontal, temporal und occipital. Die Probanden wurden an 5 Tagen im Abstand von 2-3 Tagen gemessen. Die Kopfschmerzpatienten wurden im schmerzfreien Intervall, innerhalb 24 Stunden vor der Schmerzattacke, während Kopfschmerz und innerhalb 24 Stunden nach der Kopfschmerzattacke untersucht. Die überschwellige elektrische Empfindlichkeit wurde mit einem 5 Sekunden langen Reiz $(100 \mathrm{~Hz})$ getestet, bei dessen Beginn und Ende die Schmerzintensität bewertet werden sollte.

Bei 15 gesunden Probanden änderten sich weder die Schwelle von Druck oder elektrischer Reizung noch das Rating von überschwelligen elektrischen Reizen über die 5 Testtage hinweg.

Bei 8 Spannungskopfschmerzpatienten waren die Druck- und elektrischen Schmerzschwellen generell niedriger als bei gesunden Probanden, änderten sich aber nicht über die 5 Testtage hinweg.

Sowohl gesunde Probanden als auch Spannungskopfschmerzpatienten schätzten an allen Testtagen das Ende des 5 Sekunden langen elektrischen Reizes als weniger schmerzhaft ein als den Beginn.

Bei 15 Migränepatienten waren die Schwellen von Druck und elektrische Reizung 24 Stunden vor der Migräneattacke signifikant niedriger. Während der Attacke waren die Druckschmerzschwellen erniedrigt, aber die elektrischen Schwellen tendenziell erhöht. 24 Stunden vor der Attacke war das Empfinden des 5 Sekunden langen elektrischen Reizes am Ende schmerzhafter als am Anfang. Während der Kopfschmerzattacke wurde das Ende des Reizes als sehr viel weniger schmerzhaft empfunden als der Anfang. Zusammengefasst zeigt sich eine höhere Empfindlichkeit bei Migränepatienten 24 Stunden vor der Kopfschmerzattacke. Während der Attacke könnten endogene Schmerzhemmmechanismen überwiegen. Diese erhöhte Empfindlichkeit vor dem Kopfschmerz ist spezifisch für Migränepatienten und wurde bei Spannungskopfschmerzpatienten nicht gefunden. Das heißt, die Mechanismen, die zu neuronaler Übererregbarkeit beitragen - seien sie peripher oder zentral - sind schon einige Stunden vor dem Kopfschmerzbeginn aktiv. Die gesteigerte Empfindlichkeit für Druckschmerz und elektrische Reize könnte im Unterschied zum Spannungskopfschmerz bei Migränepatienten als Frühwarnsystem vor einer kommenden Migräneattacke genutzt werden.

\section{P05.04 - Die Medikamentenpause in der Therapie des Medikamentenübergebrauch-Kopfschmerzes (MÜK): Verlauf und intervenierende Variablen}

\section{Mittermaier, A. Heinze, K. Heinze-Kuhn, A. Nielson, A. Göbel, C. Göbel, H. Gergely, H. Göbel \\ Schmerzklinik Kiel, Migräne- und Kopfschmerzzentrum, Klinik für} neurologisch-verhaltensmedizinische Schmerztherapie, Kiel, Deutschland

Einleitung: Der Medikamenten-Übergebrauch-Kopfschmerz (MÜK) ist eine häufige Komplikation in der Therapie der Migräne. Die Therapie des MÜK schließt neben der Edukation und multimodaler Therapie die Medikamentenpause (MP) für die in der Akuttherapie der primären Kopfschmerzen eingesetzten Medikamente ein. In dieser Studie sollte der Phänotyp des MÜK, der Verlauf der stationären MP, der Verlauf nach stationärer Entlassung sowie die Nachhaltigkeit der Therapie evaluiert werden. Methodik: Es wurden retrospektiv die Verlaufsdaten von 202 Patienten, die im Jahre 2012 stationär in der Schmerzklinik Kiel aufgrund des MÜK eine MP durchgeführt hatten, analysiert. Neben den klinischen Variablen wurden standardisierte Fragebögen (Kieler Schmerzkalender, Kieler Schmerzfragebogen, MIDAS, WHODAS-2, BL) in die Analyse einbezogen. Der postklinische Verlauf wurde per Telefon, E-Mail oder auf dem Postweg anhand eines standardisierten Fragebogens ermittelt. Die Daten wurden standardisiert in 857 Variablen kodiert.

Ergebnisse: Der Phänotyp des MÜK zeigt sich als Kopfschmerz von starker Intensität an in Mittel 18 Tagen im Monat. Der Schmerzcharakter ist dumpf. Der Grundschmerz wird anfallsartig überlagert von zusätzlichen Kopfschmerzattacken die über 2-3 Tage andauern und langsam einschleichend beginnen. Er wird oberflächlich als auch in der Tiefe des Kopfes verspürt und strahlt in den Nacken und den Hinterkopf aus. Die Latenz zwischen Beginn und Einleitung einer gezielten Therapie des MÜK durch eine MP beträgt im Mittel 21 Jahre. Im Analysezeitraum bis zu 36 Monate nach MP zeigte sich eine andauernde Remission des MÜK bei $78 \%$ der Patienten. Diejenigen, die während der Analgetikapause zusätzlich Kortikoide in Form von Prednisolon über 5 Tage oder Dexamethason bei Bedarf bekamen, zeigten eine signifikante Reduktion der Rebound-Kopfschmerzen während der ersten Woche der MP. Es zeigte sich ein signifikanter Zusammenhang zwischen der Dauer der MP und der Nachhaltigkeit der Behandlung. Je länger die MP durchgeführt wurde umso geringer fiel die Rückfallquote in einen erneuten MÜK aus.

Diskussion: Ein spezifischer Phänotyp des MÜK besteht im Gegensatz zu den primären Kopfschmerzen nicht. Durch eine stationäre MP mit multimodaler Therapie lässt sich selbst bei Hochrisikopatienten in einem Beobachtungszeitraum von über drei Jahren trotz sehr langer Erkrankungsdauer und komplexer psychischer und physischer Komorbidität eine sehr hohe und nachhaltige Remissionsquote erzielen. Eine möglichst lange Einhaltung der MP ist mit signifikant nachhaltigeren Langzeitergebnissen korreliert.

\section{P05.05 - Schmerztherapie bei geriatrischen Patienten - Die Perspektive der Schmerzschwester}

\section{H. Siefke, J. Weißleder, E. Sens, J. Lutz}

Zentralklinik Bad Berka GmbH, Zentrum für Interdisziplinäre Schmerztherapie, Bad Berka, Deutschland

Bei Patienten im höheren Lebensalter gehören Schmerzen zu den häufigsten Beschwerden, wodurch die Nachfrage für Schmerztherapien auch im höheren Alter in den letzten Jahren gestiegen ist. Multimorbidität und kognitive Einschränkungen der älteren Patienten erfordern allerdings ein im Vergleich zu den bisher etablierten multimodalen Therapiekonzepten eine seniorenspezifische Anpassung. Seit September 2014 wurden im Zentrum für Interdisziplinäre Schmerztherapie der Zentralklinik Bad Berka (ZIST) 96 ältere und alte Patienten mit chronischen Schmerzen im Rahmen eines solchen, neu eingerichteten Seniorenkonzeptes behandelt.

Die Arbeit der Pflege am ZIST ist dabei nicht auf die üblichen rein pflegerischen Tätigkeiten begrenzt, sondern umfasst auch einen Großteil der administrativen und organisatorischen Tätigkeiten. Darüber hinaus hält die Pflege Vorträge, zeigt und erläutert Lehrfilme und leitet Entspannungsgruppen an. Ebenso sind Verhaltensbeobachtung, Festigung von therapeutischen Inhalten und Vorgehensweisen, Förderung der Therapiemotivation, entlastende Gesprächsangebote sowie die Begleitung und Unterstützung der Patienten im Opiatentzug über 24 Stunden wichtiger Bestandteil dieser Arbeit.

Wir Schmerzschwestern des ZIST orientieren uns an den grundsätzlichen Zielen der Therapie: Aktivierung, funktionelle Verbesserung bzw. Wiederherstellung körperlicher Leistungsfähigkeit, Steigerung der Lebensqualität. Die Multimorbidität und mögliche kognitive Einschränkungen der Patienten sind dabei eine Herausforderung im alltäglichen Therapieablauf und erfordern ein angepasstes Zeitmanagement. Anleitungen und 
Unterstützungen werden mehrmals im Intervall wiederholt, um eine Festigung des Wissens und der Handhabung zu erzielen. Die Informationen für die Patienten werden kurz und leicht verständlich vermittelt, eine klare und laute Aussprache ist notwendig und deutlich mehr Zeit für Gespräche muss eingeplant werden.

Der personelle und zeitlich erhöhte Aufwand einer altersangepassten multimodalen Schmerztherapie ist, trotz des pflegerischen Mehraufwandes, aus Perspektive einer Schmerzschwester lohnenswert und sinnvoll. Die Therapieergebnisse werden in den Postern der Physiotherapie und der Psychotherapie gesondert dargestellt.

\section{P05.06 -Momentaner Inhalt und Umfang des Unterrichts zum Thema Chronischer Schmerz an deutschen Physiotherapieschulen sowie in Bachelor- und Masterstudiengängen.}

\section{B. Hägele, T. Kromer \\ SRH-Fakultät für Therapiewissenschaften, Physiotherapie, Heidelberg, Deutschland}

Hintergrund: Trotz erheblicher Wissensfortschritte und der stärker werdenden Evidenz für einen interprofessionellen Ansatz in der Versorgung und Behandlung chronischer Schmerzpatienten erhält nur ein geringer Prozentsatz der Betroffenen in Deutschland eine adäquate Versorgung. Ein Grund hierfür könnte sein, dass das Thema chronischer Schmerz bis heute kein fester Bestandteil in der Ausbildung der beteiligten Fachgruppen darstellt. So sieht die aktuell gültige Berufs- und Ausbildungsordnung für Physiotherapieschüler und -studenten explizit keinen Unterricht zum Thema chronischer Schmerz vor.

Methode: Es wurde eine Online-Umfrage zu Unterichtsumfang und -inhalt an deutschen Ausbildungseinrichtungen durchgeführt. Darauf basierend sollen die unterschiedlichen Institutionsarten wie Physiotherapieschulen, der duale Studiengang, der primäre Studiengang sowie die Masterstudiengänge miteinander verglichen werden. Weiterhin sollen die Ergebnisse mit dem Curriculum der International Association for the Study of Pain(IASP) für die Lehre der Physiotherapie abgeglichen werden. Ergebnisse: Es konnte eine durchschnittliche Übereinstimmung mit den Vorgaben der IASP von $\mathrm{MD}=52,13$ mit $\mathrm{SD}=18,57$ festgestellt werden Ein Unterschied zwischen den Institutionsarten konnte dabei nicht festgestellt werden $(p=0,07)$

Schlussfolgerung: Die aktuellen Lehrinhalte decken sich nur zu ca. 50\% mit den Angaben der IASP. Chronischer Schmerz ist ein umfangreiches Thema und es gibt bis heute große Unterschiede in Unterrichtsumfang und -inhalten der einzelnen Institutionen in der Theorie so wie in der Praxis. Eine einheitliche Vorgabe für die Lehre bezüglich dieses Themas ist wünschenswert, um einerseits den Institutionen Orientierungshilfe bei der Unterrichtsgestaltung zu bieten und andererseits eine Basis zu entwickeln, auf die die zukünftigen Physiotherapeuten bei der Therapie von chronischen Schmerzpatienten zurückgreifen können und die es ihnen ermöglicht, Chronifizierungsrisiken bei Patienten im Arbeitsalltag rechtzeitig zu identifizieren um schnellstmöglich Gegenmaßnahmen initiieren zu können.

\section{P06 - Multimodale Therapieverfahren}

\section{P06.01 - Untersuchung einer neuen, praktisch umsetzbaren und synergistischen multimodalen Intervention für die Behandlung chronischer Rückenschmerzen: Eine randomisiert-kontrollierte Multicenter Studie im Rahmen des MiSpEx-Netzwerks}

J. de Witt Huberts, A. Puschmann, P. Wippert

Universität Potsdam, Professur für Sport und Gesundheitssoziologie, Potsdam, Deutschland

Einführung: Die Entstehung chronischer Rückenschmerzen wird am besten als Wechselwirkung biologischer, psychologischer und sozialer Prozesse beschrieben. Die Wirksamkeit multimodaler Interventionsprogramme, die jeden dieser drei ätiologischen Faktoren beeinflussen sollen, bleibt weiterhin eingeschränkt. Ursächlich dafür könnte sein, dass nur wenige Programme langfristig wirken bzw. nur eingeschränkt für den alltäglichen Einsatz geeignet sind. Zudem werden häufig Elemente bestehender Programme verknüpft, aber den diesen synergistischen Effekten zugrundeliegenden Mechanismen wenig Aufmerksamkeit geschenkt. Um diese Limitationen zu überwinden, wurde eine neue multimodale Intervention entwickelt und in einer Randomisierten Kontrollierten Studie im Rahmen des MiSpEx-Netzwerks sowohl mit einer unimodalen sensomotorischen Intervention als auch einer Kontrollgruppe verglichen.

Methoden: Insgesamt 660 Probanden wurden einer von 3 Gruppen zugeordnet (Kontrollgruppe: $n=216$, Unimodal: $n=222$, Multimodal: $n=222$ ). Die beiden Interventionsgruppen führten über 3 Wochen hinweg ein center-based Training durch, gefolgt von einer 9-wöchigen home-based Phase. In der multimodalen Intervention wurde das sensomotorische Training der unimodalen Gruppe um kognitiv-verhaltenstherapeutische sowie psychophysiologische Techniken erweitert. Über 6 Monate hinweg wurden zu 5 Messzeitpunkten Schmerzintensität und Beeinträchtigung sowie schmerzrelevante psychosoziale Faktoren erfasst.

Ergebnisse: Sowohl Schmerzintensität, Beeinträchtigung als auch schmerzbezogene Kognitionen wurden in beiden Interventionsgruppen nach der center-based Phase im Vergleich zur Kontrollgruppe reduziert, wobei sich die Interventionsgruppen nicht unterschieden.Nach der home-based Phase dagegen zeigte sich, dass diese Veränderungen in der multimodalen Interventionsgruppe anhaltender bzw. stärker ausgeprägt waren als in der unimodalen oder der Kontrollgruppe, welches eine nachhaltigeren Einfluss des multimodalen Programms auf Rückenschmerzen nahelegt.

Diskussion: Mit der hier vorgestellten Studie konnte zunächst die Machbarkeit der neu entwickelten multimodalen Intervention belegt werden. Weiterhin wurden nachhaltigere Effekte der multimodalen Intervention auf Rückenschmerzen gezeigt. Die vorgestellten Effekte sind besonders für die Langzeittherapie von Rückenschmerzen i.S. der Unterstützung heimbasierter Selbsthilfe mit gleichzeitiger Behandlung psychosozialer Faktoren relevant.

\section{P06.02 - Faktorenstruktur der DASS-21 bei chronischen Schmerzpatienten}

O. Kuhnt ${ }^{1}$, I. Haase ${ }^{2}$, T. Helmer ${ }^{3}$, K. Klimczyk ${ }^{1}$

${ }^{1}$ m\&i-Fachklinik Enzensberg, Interdisziplinäres Schmerzzentrum, Hopfen am See, Deutschland, ${ }^{2}$ m\&i-Klinikgruppe Enzensberg, Forschung, Entwicklung und Qualitätssicherung, Hopfen am See, Deutschland, ${ }^{3} \mathrm{~m} \& \mathrm{i}-$ Fachklinik Enzensberg, Interdisziplinäres Schmerzzentrum, Hopfen am See, Deutschland

Fragestellung: Der neue Schmerzfragebogen der DSG beinhaltet als Depressionstest die deutsche Version der „Depression Anxiety and Stress Scale“ - DASS-21 (Deutsche Schmerzgesellschaft 2012). Der DASS ist ein lizenzfreier Selbstbeurteilungsfragebogen mit 21 Items zur Erfassung von Depressivität, Angst und Stressbelastung mit jeweils sieben Items. Ihm wurden bisher gute psychometrische Eigenschaften hinsichtlich Reliabilität und Validität attestiert (u. a. Nilges \& Esau 2015), aber auch komplexe Ladungen in Faktorenanalysen (Poliakov 2015). Vor diesem Hintergrund untersuchten wir die Faktorenstruktur der DASS-21, angewendet in einem Kollektiv chronischer Schmerzpatienten.

Methodik: Zwischen Mai 2014 und Dezember 2015 füllten 581 Patienten mit chronischen Schmerzen (Chronifizierungsgrad II oder III nach Gerbershagen) vor Aufnahme in ein Interdisziplinäres Schmerzzentrum einen Fragebogen aus, der u. a. den DASS-21 enthielt. Es wurde eine Hauptkomponentenanalyse mit Vorgabe von 3 Faktoren durchgeführt, die Rotation erfolgte nach der Methode Varimax mit Kaiser-Normalisierung (SPSS 21). Ergebnisse: Die untersuchte Gruppe chronischer Schmerzpatienten $(\mathrm{N}=581)$ war überwiegend weiblich $(70 \%)$ und im Mittel 54 Jahre alt (18-84; SD =11,3). Die durchschnittlichen DASS- Werte bei Aufnahme betrugen 7,8 $(\mathrm{SD}=5,2)$ für die Skala Depression, 5,2 $(\mathrm{SD}=4,3)$ für Angst und $9,1(\mathrm{SD}=4,8)$ für Stress. Unter Vorgabe von drei zu extrahierenden Faktoren ließen sich die drei Dimensionen mit einer erklärten Gesamtvarianz von 62,1\% nur mit Einschränkungen reproduzieren. Die Skala De- 
pression konnte mit hohen Ladungen $(>0,6)$ bei sechs von sieben Items weitgehend bestätigt werden. Lediglich das Item 17 fällt etwas ab. Bei der Skala Angst luden vier Faktoren eindeutig auf den erwarteten Faktor, drei Items wiesen Doppelladungen auf. Für die Skala Stress ließen sich fünf Items reproduzieren, zwei Items luden höher auf den Faktor Depression. Schlussfolgerung: Der DASS-21 erwies sich in der Anwendung bei Schmerzpatienten bisher als ökonomisch und praktikabel. Die Skalen zeigten überwiegend gute psychometrische Eigenschaften (Nilges \& Esau 2015, Kuhnt et al. 2015). Unsere aktuelle Untersuchung zeigt aber auch Schwächen der faktoriellen Validität: Einzelne DASS-Items wiesen komplexe Ladungen auf (zusätzliche substanzielle Ladungen > .30 auf einen zweiten Faktor und teilweise dritten Faktor). Die Skalen Angst und Stress enthalten inhaltlich nicht immer abgrenzbare Einzelitems. Für den Einsatz bei chronischen Schmerzpatienten wäre deshalb zu überlegen, diese Skalen zu überarbeiten oder den DASS auf die 7 Depressions-Items zu beschränken.

\section{Literatur}

Deutsche Schmerzgesellschaft (2012) Deutscher Schmerzfragebogen. Handbuch. Überarbeitete Version 2012.2

Kuhnt O, Haase I, Klimczyk K (2015) Erfahrungen mit der DASS-21 in der stationären multimodalen Schmerztherapie. Schmerz 29 (Suppl. 2):S82

Nilges P, Esau C (2015) Die Depressions-Angst-Stress-Skalen. Der DASS - ein Screeningverfahren nicht nur für Schmerzpatienten. Schmerz 29: 649-657

Poliakov B (2015) Chronischer Schmerz: Klinische Diagnostik von Angst mit DASS-21 und Depression mit BDI-II. Unveröffentlichte Masterarbeit im Masterstudiengang Psychologie, Universität Bamberg

\section{P06.03 - Selbsteinschätzung des Einflusses auf den Schmerz nach einer multimodalen Schmerztherapie. 1-Jahres- Verlausbeobachtung.}

C. Schön, C. Geiß, P. Mattenklodt, N. Grießinger

Universitätsklinikum, Schmerzzentrum, Erlangen, Deutschland

Hintergrund: Multimodale teilstationäre Gruppen-Behandlungsprogramme haben sich als wirksame Therapieform bei Patienten mit chronischen Schmerzen etabliert. Die Behandlung hat das Ziel, Schonhaltungen und Hilflosigkeit abzubauen und körperliche und psychosoziale Aktivität und Selbsteffizienz zu steigern [1]. Ziel der Untersuchung war es herauszufinden, ob der Teilaspekt des Therapieerfolges „Abbau von Hilflosigkeit und Steigerung von Selbsteffizienz" im Rahmen einer multimodalen Schmerztherapie signifikant gesteigert und über einen Zeitraum von 12 Monaten aufrechterhalten werden kann.

Methodik: In die Untersuchung wurden 162 Patienten eingeschlossen, die zwischen Oktober 2012 und Februar 2015 an einer teilstationären multimodalen Schmerztherapie am Schmerzzentrum des Universitätsklinikums Erlangen teilnahmen. Wir haben diese Patienten gebeten, zu t0 (Screening), zu t1 (Gruppenende) und zu t2 (ein Jahr nach Therapieende) ihren Einfluss auf die Schmerzen auf einer Skala von 0 bis 10 einzuschätzen: Wie gut konnten Sie in den letzten 14 Tagen Ihren Schmerz selbst beeinflussen? $(0=$,gar nicht“, $10=$,sehr gut“).

Die Überprüfung von Mittelwertunterschieden erfolge durch den T-Test für abhängige Stichproben.

Ergebnisse: Die Selbsteinschätzung des Einflusses auf den Schmerz verbesserte sich im Rahmen der Therapie signifikant $(p<.05)$ von durchschnittlich 3,0 (SD 2,1) auf 5,8 (SD 2,0). Nach 12 Monaten lag sie bei durchschnittlich 5,6 (SD 2,3). Die Verbesserung konnte somit weitgehend aufrechterhalten werden.

Diskussion: Seit Oktober 2012 erfassen wir die Selbsteinschätzung des Einflusses auf die Schmerzen bei der Erstvorstellung in unserem Schmerzzentrum, am Ende des Therapieprogramms und ein Jahr danach. Unserer Ansicht nach stellt die Frage ein geeignetes Instrument dar, um wichtige Teilaspekte des Therapieerfolges zu erfassen. Die Fragestellung ist für die Patienten leicht zu verstehen und nachzuvollziehen. Außerdem korreliert sie mit den Subskalen „Kompetenzerleben“, „Schmerzbedingte Hilflosigkeit und Depression“, „Schmerzbedingte Angst“, „Handlungsplanungskompetenz", „Kognitive Umstrukturierung “ und „Schmerzbedingter Är ger" des Fragebogens zur Erfassung der Schmerzverarbeitung (FESV) [2].

\section{Literatur}

1. Nagel et al (2012) Struktur- und Prozessqualität multimodaler Schmerztherapie. Schmerz 26(6): 661-669

2. Schön et al (2012) Selbsteinschätzung des Einflusses auf den Schmerz zur Erfolgsbeurteilung in der multimodalen Schmerztherapie. Schmerz 26(Sonderheft 1):94

\section{P06.04 - Vorläufige Ergebnisse einer randomisiert-kontrollierten Studie zur Wirksamkeit der Schmerzprovokation bei Jugendlichen mit chronischen Schmerzen}

F. Flack, M. Dobe, B. Zernikow, J. Wager

Deutsches Kinderschmerzzentrum, Vestische Kinder- und Jugendklinik Datteln, Universität Witten Herdecke, Datteln, Deutschland

Hintergrund: Angst vor Schmerzen wird in etablierten Theorie-Modellen als zentraler aufrechterhaltender Faktor für chronische Schmerzen diskutiert. Diese Angst kann zu vermehrtem Vermeidungsverhalten führen und dadurch erhöhte Beeinträchtigung im Alltag zur Folge haben. Therapeutische Interventionen, die gezielt diese Angst reduzieren, sind daher gefragt. Die sogenannte „Schmerzprovokation“ ist eine Form der interozeptiven Expositionstherapie bei der Kinder und Jugendliche zur Schmerzfokussierung und Schmerzmodulierung angeleitet werden. In der vorliegenden randomisiertkontrollierten Therapiestudie (RCT) soll die Wirksamkeit der Schmerzprovokation zur Reduktion der Angst vor Schmerz untersucht werden.

Methode: Insgesamt werden - basierend auf einer Stichprobenumfangsplanung $-N=126$ chronisch schmerzkranke Jugendliche im Alter von 1118 Jahren für die RCT rekrutiert. Die Jugendlichen werden randomisiert zwei Interventionen zugewiesen: der „Schmerzprovokation“ oder einem „Entspannungsverfahren“ (Progressive Muskelrelaxation). Beide Therapieinterventionen finden insgesamt $5 \mathrm{Mal}$ adjunktiv im Rahmen einer 3- bis 4-wöchigen stationären interdisziplinären Schmerztherapie statt. Die Angst vor Schmerzen wird mit der validierten „Skala zur Erfassung schmerzbezogener Angst von Kindern und Jugendlichen" (SESA-KJ) bei stationärer Aufnahme sowie bei Entlassung gemessen. Die SESA-KJ kann einen Gesamtscore zwischen 0-59 erzielen und umfasst die Subskalen „Angst“ (Wertebereich 0-36) und „Vermeidung“ (Wertebereich 0-24).

Zwischenergergebnisse: Bislang wurden $n=80$ Jugendliche im Alter von durchschnittlich 14,7 Jahren $(S D=1,7)$ - davon 56 Mädchen $(70 \%)$ und 24 Jungen (30\%) - in die RCT eingeschlossen. Zum Aufnahmezeitpunkt lag die Subskala „Angst" bei durchschnittlich 13,3 (SD=7,1), die Subskala „Vermeidung" erzielte einen Durchschnittswert von 10,4 $(S D=4,4)$ und der Gesamtwert betrug durchschnittlich 23,7 $(S D=10,3)$. Bei Entlassung zeigten sich signifikant reduzierte Werte sowohl für den Gesamtscore $(t(76)=5,982, p<.001)$ als auch für die Subskalen „Angst“ $(t(76)=3,866$, $p<.001)$ und „Vermeidung“ $(t(76)=7,716, p<.001)$. Der Vergleich der beiden Therapieoptionen "Schmerzprovokation“ und „Entspannungsverfahren“ zeigt beim aktuellen Rekrutierungsstand keine signifikanten Gruppenunterschiede im Gesamtscore $(t(76)=-0,714, p>.477)$ und den Subskalen „schmerzbezogene Angst" $(t(76)=-0,040, p>.968)$ und „Vermeidung" $(t(76)=-1,720, p>.089)$.

Schlussfolgerung: Die vorläufigen Ergebnisse zeigen eine Reduktion der Angst vor Schmerz sowohl in der Therapiebedingung "Schmerzprovokation“ als auch bei „Entspannungsverfahren“. In Bezug auf die Vermeidungskomponente zeigt sich bei Entlassung eine tendenziell stärkere Reduktion in der „Schmerzprovokation“. Bisher wurde die Patientenzahl, die in der Stichprobenumfangsplanung berechnet wurde, noch nicht erreicht. Somit ist eine endgültige Bewertung der spezifischen Wirksamkeit der Schmerzprovokation in Bezug auf die Angst vor Schmerzen zum jetzigen Zeitpunkt nicht möglich.

\section{P06.05 -Qigong bei chronischen Schmerzen}

\section{A. Spudeit, E. Metje, T. Brinkschmidt}

Algesiologikum, Abteilung für interdisziplinäre Schmerztherapie Harlaching, München, Deutschland

Einleitung: Qigong gehört zu den achtsamkeitsorientierten Bewegungsformen, bei der fließende Bewegungen mit Aspekten der Atmung und der 
Aufmerksamkeit in Verbindung gebracht werden. Die Wirksamkeit von Qigong bei chronischen Schmerzen ist bisher nicht eindeutig belegt, dennoch kommt es häufig als Behandlungsbaustein in multimodalen Therapieprogrammen zur Anwendung. Derzeit weiß man wenig darüber, wie Qigong von Patienten mit chronischen Schmerzen aufgenommen wird. Fragestellung:Wie wird Qigong von Patienten im Rahmen eines multimodalen Schmerzbewältigungsprogrammes aufgenommen?

Methode: Anhand eines selbstentwickelten Fragebogens wurde die Einstellung der Patienten zu dem Qigong-Therapieangebot erhoben. Konkret wurde gefragt nach Vorerfahrung, nach "Gefallen“ und „Nicht-Gefallen“ mit der Möglichkeit, dies jeweils genauer zu beschreiben. Der Fragebogen wurde im Verlauf erweitert um Fragen nach „Was können Sie aus dem Qigong für sich selber mitnehmen“ und „Möchte Sie Qigong weiterüben“. Die Beantwortung des Fragebogens war freiwillig. Für die Auswertung wurden ähnliche Antworten gruppiert. Die Ergebnisse werden beschreibend dargestellt.

Ergebnisse: Es wurden bisher 41 Fragebögen ausgewertet, davon 17 mit erweitertem Fragenumfang. 32 Patienten kannten Qigong bisher noch nicht. Gefragt nach dem „Gefallen“ gaben 41 Patienten insgesamt 147 Antworten, aus denen 95 verschiedene Antworten nach folgenden Aspekten kategorisiert werden konnte: Geistige Aktivität, Gefühl und gefühlte Wirkung, Gefühl/Wahrnehmung des Körpers, Atmung, Gefühl für Bewegung, Gefühl der Ruhe und Entspannung, Gefühl/Wahrnehmung für ein Selbst und Setting. 13 Patienten gaben zusätzlich 14 Aspekte des „Nicht-Gefallens“ an. 17 Patienten füllten den erweiterten Fragebogen aus, davon konnten sich 14 vorstellen, Qigong weiter zu üben.

Diskussion: Alle an der Befragung teilnehmenden Patienten haben Qigong positiv aufgenommen. Dies wird mit einem großen Spektrum an sowohl körperbezogenen, als auch gefühlsbezogenen und umweltbezogenen Angaben beschrieben. Möglicherweise kann Qigong als integrative Übungsform einen guten Zugang zur Wahrnehmung dieser verschiedenen Modalitäten herstellen. Die verschiedenen Aspekte des Gefallens könnten sich ferner positiv auf eine Therapieadhärenz auswirken. Welchen Stellenwert Qigong in multimodalen Schmerztherapieprogramm einnehmen kann, bleibt Gegenstand weiterer Untersuchungen.

\section{P06.06 - Sensitivität und Spezifität von Screeningfragebogen für Depressivität in der interdisziplinären multimodalen Schmerztherapie}

\section{S. Koch ${ }^{1}$, H. Berth ${ }^{2}$, R. Sabatowski', U. Kaiser ${ }^{1}$}

'Universitätsklinikum Dresden, UniversitätsSchmerzCentrum, Dresden, Deutschland, ${ }^{2}$ Universitätsklinikum Dresden, Psychosoziale Medizin und Entwicklungsneurowissenschaften, Dresden, Deutschland,

Hintergrund: In der Fachliteratur wird häufig ein signifikant erhöhtes, gemeinsames Auftreten von Depressivität und chronischen Schmerzen berichtet. Ebenso wird jedoch auch von einer Überschätzung der Prävalenzen ausgegangen und vor einer Überbewertung der Befunde gewarnt, da bestimmte unspezifische Symptome (z. B. Schlafprobleme, leichte Ermüdbarkeit) sowohl Begleiterscheinungen der Depression als auch des chronischen Schmerzes darstellen können und es somit einen großen Überschneidungsbereich gibt.

Methotik: Im Rahmen einer Untersuchung an einer tagesklinischen Stichprobe von 98 Patienten mit chronischen Schmerzen des UniversitätsSchmerzCentrums (USC) Dresden wurde die methodische Qualität von vier verschiedenen Screeninginstrumenten zur Erfassung depressiver Symptomatik exploriert. Die Daten wurden von Oktober 2014 bis März 2015 erhoben. In die Untersuchung wurden die Allgemeine Depressionsskala (ADS), das Beck-Depressionsinventar (BDI), die Kurzform der Depression-Angst-Stress-Skala (DASS-21) sowie die Hospital Anxiety and Depression Scale (HADS-D) einbezogen. Neben der Ermittlung der Prävalenzraten depressiver Symptome wurden die Fragebögen mittels Receiver-Operating-Characteristic-Methode (ROC) hinsichtlich ihrer Sensitivität und Spezifität analysiert. Als Außenkriterium wurden dafür die psychischen Diagnosen genutzt, welche durch das behandelnde Team des USC gestellt wurden.
Ergebnisse: Es zeigte sich eine große Heterogenität bezüglich der anhand der Fragebögen ermittelten Punktprävalenzraten depressiver Symptome (DASS-21: 20,4\%, ADS: 29,6\%, HADS-D: 39,8\%, BDI: 51,0\%). Die Auswertung der psychischen Diagnosen ergab, dass bei 33,7\% der untersuchten Stichprobe eine depressive Störung mit aktuell relevanter Ausprägung der Symptomatik vorlag. Hinsichtlich der Sensitivität und Spezifität erreichte lediglich das BDI akzeptable Kennwerte (Sens.: $75 \%$, Spez.: 61,5\%). Auffallend niedrige Sensitivitätswerte wiesen hingegen die ADS und die DASS-21 (Sens.: jeweils 36,4\%) bei hoher Spezifität (ADS: 83,1\%, DASS21: 87,7\%) auf. Für die HADS-D ergab sich ebenfalls eine höhere Spezifität $(73,8 \%)$ als Sensitivität $(63,6 \%)$.

Schlussfolgerung: Dem Anspruch einer möglichst sehr hohen Sensitivität konnte in der Untersuchung an der tagesklinischen Stichprobe keiner der Fragebögen wirklich gerecht werden. Das beste Verhältnis von Sensitivität und Spezifität wies das BDI auf, welches jedoch das Vorkommen depressiver Symptomatik in der untersuchten Stichprobe deutlich überschätzte. Besonders hervor stach zudem die ungenügende Sensitivität der DASS21 sowie der ADS. Die Untersuchung bestätigt erneut, dass der Einsatz von Screeningfragebögen nur Hinweise auf das Vorliegen einer depressiven Symptomatik gibt, die im Rahmen einer ausführlichen Diagnostik abzuklären ist.

\section{P06.07 - Zusammenhang zwischen Einschätzung des Therapieerfolges und Veränderungen in Bezug auf psychische Variablen nach interdisziplinärer multimodaler Schmerztherapie (IMST)}

L. Taubert, J. Böhme, M. Klotzsche, B. Rust, M. Schiller, R. Sabatowski,

U. Kaiser

Universitätsklinikum Dresden, UniversitätsSchmerzCentrum, Dresden, Deutschland

Hintergrund: In Voruntersuchungen konnte ein Zusammenhang zwischen der subjektiven Einschätzung des Therapieerfolges nach MMST am USC und erreichten Veränderungen auf spezifischen Parametern nachgewiesen werden. In der vorliegenden Studie soll dieses Konzept nun auf weitere, vorwiegend psychische Parameter ausgeweitet werden.

Methodik: $N=192$ Patienten der MMST am USC wurden im Zeitraum von Februar 2014 bis November 2015 zu zwei Messzeitpunkten (T1: Therapiebeginn; T4: Halbjahreskatamnese) untersucht. Das Durchschnittsalter der Patienten lag bei 51,32 Jahren ( $\mathrm{SD}=11,72), 73,4 \%$ waren weiblich und 54,2\% berufstätig. Infolge einer zu T4 vorgenommenen, subjektiven, globalen Selbsteinschätzung des Therapieerfolges auf einer 5-stufigen Ratingskala, wurden die Patienten in 3 Gruppen eingeteilt (1: sehr gut/gut; 2: zufriedenstellend; 3: weniger gut/schlecht) und es wurden Gruppenunterschiede in Bezug auf zwischen T1 und T4 erreichte Veränderungen untersucht. Dabei waren die Katastrophisierung des Schmerzerlebens (PCS), das psychische Wohlbefinden (FW-7) sowie Depressivität, Ängstlichkeit und Stresserleben (DASS) von Interesse. Mithilfe von SPSS 23, $0^{\circ}$ wurden nach der Berechnung von Differenzwerten zwischen T1 und T4 einfaktorielle Varianzanalysen sowie Rangvarianzanalysen (Kruskal \& Wallis) bei einem korrigierten Alpha-Niveau von $\alpha_{\text {adj. }}=.01$ eingesetzt und anschlieBende post-hoc-Analysen durchgeführt.

Ergebnisse: Es ergaben sich signifikante Mittelwertunterschiede zwischen den 3 Gruppen bezüglich Veränderungen auf der PCS zwischen T1 und $\mathrm{T} 4(\mathrm{~F}=15,54 ; p<.001)$, wobei sich Pat. der Gruppen 1 und 2 verbesserten, während Patienten der Gruppe 3 eine leichte Verschlechterung zeigten $\left(\mu_{\text {DiffGr.1 }}=6,95, \mu_{\text {DiffGr.2 }}=3,84, \mu_{\text {DiffGr.3 }}=-2,93\right)$. Die Effektstärke beträgt $\eta_{\text {part. }}^{2}=.186$ (großer Effekt). Bezüglich der übrigen Parameter (FW-7, DASS) ergaben sich keine signifikanten Mittelwertunterschiede zwischen den Gruppen.

Schlussfolgerung: Die subjektive, globale Therapieeinschätzung lässt sich in Zusammenhang bringen mit Veränderungen auf der „PCS“, nicht jedoch mit Veränderungen auf den Messinstrumenten „FW-7“ oder „DASS“. Das bedeutet, dass Patienten, die die Therapie als erfolgreich einschätzten, auch signifikant stärkere Verbesserungen im Bereich der Katastrophisierung des Schmerzerlebens erreichten. Da die fehlenden Zusammenhän- 
ge mit dem „FW-7“ sowie der „DASS“ auf die geringe Fallzahl und mangelnde Teststärke zurückgeführt werden könnten, sind weitere Analysen erforderlich.

\section{P06.08 - „Conditioned pain modulation“ als prognostischer Parameter für die Effektivität der multimodalen Schmerztherapie.}

F. Lienau' ', D. Fischer' ${ }^{2}$, F. Wallburg ${ }^{2}$, M. Rossi' ${ }^{2}$, J. Moll' ${ }^{2}$, A. Binder ${ }^{3}$

${ }^{1}$ Asklepios Klinik Nord - Heidberg, Zentrum für interdisziplinäre Schmerztherapie, Hamburg, Deutschland, ${ }^{2}$ Zentrum für interdisziplinäre Schmerztherapie, Hamburg, Deutschland, ${ }^{3}$ Universitäts-Klinikum Campus Kiel, Klinik für Neurologie, Kiel, Deutschland

Einleitung: „Conditioned pain Modulation“ (CPM) untersucht die individuelle zentrale absteigende Schmerzhemmung. In der multimodalen Schmerztherapie (MMST) wird auf diversen therapeutischen Ebenen Einfluss auf Schmerzempfindung, Schmerzwahrnehmung und Schmerzverarbeitung genommen. Die klinische Beobachtung suggeriert, das die Effekte der Therapie häufig über die Möglichkeiten der Summe der einzelnen Therapiebausteine hinaus gehen, zum Teil auch nicht. Wir haben die Patienten, die für die stationäre MMST vorgesehen waren und ihr Einverständnis gegeben haben, vor und nach der stationären Therapie untersucht. Mit der Untersuchung haben wir zwei Fragen verfolgt. Zum einen kann die CPM Patienten identifizieren, welche von der MMST besonders profitieren? Und zum anderen ändert sich die zentrale Schmerzhemmung (CPM) durch die MMST?

Methoden: Im Zeitraum vom Mai 2015 bis Mai 2016 wurden 127 Patienten (72\% Frauen, im Mittel 55,3 J alt) eingeschlossen, 29 Dropouts. Die Behandlung umfasste 10 stationäre Therapietage mit insgesamt ca. 23 Therapieeinheiten. Die Therapien umfassten Physiotherapie in Gruppen- und Einzeltherapie, Psychotherapie in Gruppen- und Einzeltherapie, Entspannungstherapie, medizinische Informationsvorträge, tägliche ärztliche Visite. Vor und nach der MMST wurden folgende Untersuchung durchgeführt: CPM, QST (WUR, HPT, PPT), PGIC/CGIC, HAMD, SF-12, SSS MOS SS, PainDetect.

Ergebnisse: Bei Aufnahme zeigten 71 Patienten eine pathologische zentrale Schmerzhemmung in der CPM Messung und 26 eine physiologische. Bei der Betrachtung der numerischen Analog Skala (NAS) der letzten 3 Tage konnten die Patienten signifikant von der MMST profitieren $(p=0,000)$. Beide Gruppen profitierten signifikant, aber die Patienten mit der physiologischen zentralen Schmerzhemmung profitierten signifikant deutlicher $(p=0,028)$. Bei den psychologischen Scores waren die beiden Gruppen zum Aufnahmezeitpunkt vergleichbar. Beide Gruppen profitierten im Depressionsscore des HADS von der Therapie $(p=0,000)$, wobei auch hier die Gruppe mit physiologischem CPM signifikant deutlicher profitierte $(p=0,028)$.

Fazit: Die CPM gibt eine prognostische Aussage zur Effizienz der MMST. Patienten mit einer physiologischen zentralen Schmerzverarbeitung profitieren deutlicher als die mit einer pathologischen zentralen Schmerzhemmung. Möglicherweise profitieren die Patienten mit einer pathologischen $\mathrm{CPM}$ von einer länger andauernden Therapie.

Eine Änderung bzw. Besserung der CPM durch die MMST konnte nach 10 Therapietagen nicht nachgewiesen werden.

Die Studie dauert noch an. Für Subgruppenanalysen ist die Patientenzahl noch zu gering.

Die Studie wird von der Asklepios Forschungsförderung unterstützt.

P06.09 - Vom Chaos zum Licht - Ergebnisse einer qualitativen Einzelfallstudie zur kunst- und psychotherapeutischen Zusammenarbeit in der Multimodalen Schmerztherapie

K. Wendschuh, B. Schönbach, R. Sabatowski, U. Kaiser

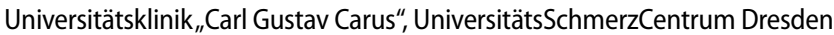

Einleitung: Am UniversitätsSchmerzCentrum Dresden ist Kunsttherapie in das multimodale Behandlungsprogramm integriert. Insbesondere zwischen Kunst-und Psychotherapie werden die vorhandenen inhaltlichen
Überschneidungen genutzt. Erlebnisorientierte kunsttherapeutische Methoden stellen die Möglichkeit einer Annäherung zu Schmerz-und darüber hinaus Lebensthemen dar, die psychotherapeutisch oftmals noch nicht unmittelbar zugänglich sind. Durch eine intensive Zusammenarbeit zwischen Kunst-und Psychotherapeuten kann der Behandlungsprozess effektiver, ganzheitlicher und individueller gestaltet werden. Anhand eines Einzelfalls soll der Verlauf und die Zusammenarbeit zwischen beiden Therapierichtungen dargestellt werden.

Methodik: Qualitative Einzelfalldarstellung des Therapieverlaufs im Rahmen einer multimodalen teilstationären Therapie über 4 Wochen und einer Wiederholungswoche. Männlicher Patient, Migräne, 57 Jahre, gelernter Pädagoge, Vertreter im Außenhandel eines Unternehmens.

Ergebnis: In der Kunsttherapie zeigte sich beim Patienten zu Behandlungsbeginn ein diffuser und abstrakter Ausdruck. Im psychotherapeutischen Einzelkontakt fiel bei der gemeinsamen Betrachtung der Bilder im Hinblick auf die Lebenssituation betreffenden Themen des Patienten ein deutliches Überforderungserleben auf. Im weiterführenden, parallel angelegten kunst- und psychotherapeutischen Behandlungsprozess konnten einzelne Themen und deren Zusammenhang identifiziert und klarer herausgearbeitet werden. Dadurch gelang es dem Patienten hinsichtlich dieser Themen zunehmend Prioritäten zu setzen und diese auf seinen Lebenskontext zu übertragen. Darauf aufbauend konnte er in der Kunsttherapie neue Perspektiven und damit in der Psychotherapie konkrete Veränderungsziele entwickeln und später umsetzen. Der Verlauf wird sowohl aus kunsttherapeutischer Sicht mit konkreten Bildern dargestellt und in Zusammenhang zum psychotherapeutischen Geschehen gesetzt.

Schlussfolgerung: Die ganzheitliche Betrachtung des Schmerzerlebens, das über die reine Bewältigung hinaus in den Lebenskontext des Patienten weist, wird durch erlebensorientierte Maßnahmen unterstützt und gefördert. Durch die enge Verzahnung mit psychotherapeutischen Interventionen werden ansonsten schwer zugängliche Themen greifbar und stehen damit einer Verarbeitung unmittelbarer zur Verfügung.

\section{P07 - (Um)denken erwünscht}

\section{P07.01 - Schmerzintensität in der interdisziplinären multimodalen Schmerztherapie (IMST)- erste Ergebnisse einer quantitativen Untersuchung zu psychometrischen Eigenschaften (VAPAIN)}

U. Kaiser', K. Neustadt' , A. Preißler', R. Scharnagel', J. Schmitt', R. Sabatowski ${ }^{1}$

'Universitätsklinikum Dresden, UniversitätsSchmerzCentrum, Dresden, Deutschland, ${ }^{2}$ Universitätsklinikum Dresden, Zentrum für evidenzbasierte Gesundheitsversorgung, Dresden, Deutschland

Einleitung: Die Veränderung von Schmerzintensität (SI) dient als Parameter für die Erfassung des Therapieerfolgs. Der Einsatz solcher Skalen wirft aktuell methodische Probleme auf- in der Qualität von Studien zur Prüfung ihrer psychometrischen Eigenschaften sowie in der Konstruktion der Skalen. Sowohl Validität als auch Reliabilität werden aktuell kritisiert. Ein systematischer Literaturreview offenbarte eine große Anzahl an z. T. sehr unterschiedlichen Skalen zur Messung von SI. Ziel der Untersuchung war, eine Auswahl dieser Skalen bezüglich der konvergenten Validität sowie ihrer Sensitivität zu untersuchen. Die Studie wurde im Rahmen des VAPAIN-Projektes durchgeführt und beinhaltet eine multizentrische Studie in Einrichtungen mit IMST. Die dargestellten Ergebnisse sind eine Voranalyse anhand eines Teiles der eingeschlossenen Stichprobe.

Methodik: Eingeschlossen wurden 67 Patienten mit multiplen Schmerzen (Alter MW 47; SD 11,27; 66\% weiblich; 46\% MPSS II) einer IMST(Dresden), die zu zwei Zeitpunkten (Therapiebeginn, Wiederholungswoche; Abstand 14 Wochen) (Sub)Skalen zur Messung von SI (NRS, von Korff, Obstacles for return to work (ORQ), McGill, Multidimensional Pain Inventory (MPI)) beantworteten. Die Skalen repräsentieren exemplarisch eindimensionale numerische und verbale Messinstrumente (NRS SI 
McGill), eindimensionale Summenskalen (von Korff) sowie mehrdimensionale Summenskalen (MPI). Die Auswertung (Normalverteilung liegt vor) erfolgte anhand von Korrelationen (Pearson; konvergente Validität), ALM sowie Regressionen (SPSS $21,0, \alpha_{\mathrm{korr}}<0,004$, Bonferroni). Der Standardmessfehler sowie Effektstärken wurden bestimmt (Sensitivität).

Ergebnisse: NRS SI korrelierte niedrig mit anderen Skalen $(r \leq 0,5)$, bis auf den MPI $(r \geq 5, p<0,000)$. Alle Summenskalen dagegen korrelierten hoch miteinander $(\mathrm{r}>0,8, p \leq 0,000)$, unabhängig von ihrer Dimensionalität. Insgesamt lag die Therapieveränderung höher als der Standardmessfehler $\left(1,4<\gamma_{\mathrm{T} 3-\mathrm{T} 1}<5\right)$. Allerdings zeigte sich bei keiner Skala eine signifikanten Veränderung $(p \leq 0,006)$ über den Therapieverlauf, bei heterogenen, kleinen Effekten $\left(0,001<\eta_{\text {part }}^{2}<0,06\right)$ und mangelnder Teststärke $(1-\beta<0,8)$. Zusammenfassung: Die Ergebnisse sind als Voranalyse zu werten und können aufgrund der geringen Stichprobe und des deutlich reduzierten Alphaniveaus nicht generalisiert werden. Es zeigen sich jedoch Hinweise auf methodische Unterschiede in der Anwendung von Skalen zur Messung der SI in Abhängigkeit von deren Beschaffenheit. Eindimensionale Skalen zeigten keine konvergente Validität mit Summenskalen, die jedoch untereinander unabhängig von ihrer Dimensionalität hohe Konvergenzen aufwiesen. Veränderungssensitivität, die hinsichtlich der Skalen sehr unterschiedlich ausfiel, kann für alle Skalen außer den ORQ angenommen werden. Zusammenfassend ergeben sich Hinweise auf die Bedeutung von Skalenkonstruktionen sowie eine unterschiedliche psychometrische Güte der untersuchten Skalen für die Erfassung von Therapieerfolg in der IMST.

\section{P07.02 - Basisprogramm Ernährung und Bewegung Grundlegendes und Nachhaltiges zum täglichen Umgang mit chronischem Schmerz}

\section{U. Hamberger ${ }^{1}$}

\section{'Klinikum Landsberg, Schmerzzentrum, Kaufering, Deutschland}

Chronischer Schmerz steht mit somatischen und psycho-sozialen Komorbiditäten in Wechselwirkung. Hierfür scheinen auch entzündungsfördernde Zytokine als gemeinsame Kommunikationsplattform, mitverantwortlich zu sein. Ein wesentlicher Ursprungsort dieser ist in Makrophagenansiedlungen in unmittelbarer Nachbarschaft viszeraler Fettansammlungen zu finden. (Ghazarian et al. 2015; Wensvenn et al. 2015) Die hierdurch hervorgerufene unterschwellige, aber dauerhafte Aussendung proinflammatorischer Zytokine, kann in Form einer sogenannten „Silent Inflammation“ zu Insulinresistenz, Metabolischem Syndrom, Depression und offensichtlich auch, zentralen Sensibilisierungsprozessen beitragen (Andrade et. al 2010; Leung u. Cahill 2010)

Körperliche Aktivierung (Pedersen 2011) und ballaststoffreiche Ernährung mit niedriger glykämischer Last (Buyken et al. 2014) wirken sowohl der Fettansammlung, als auch einer Silent Inflammation entgegen. Bewegungsmangel und Ernährung mit hoher glykämischer Last, begünstigen die Verschiebung der Körperkomposition in Richtung viszerales Fett mit niedriggradiger systemischer Entzündungslage.

Körperliche Aktivierung scheint ein grundlegendes Instrument darzustellen, der Chronifizierung von Schmerz entgegen zu wirken. In jüngerer Zeit verdichten sich die Hinweise, dass hierbei auch der Muskulatur als sekretorisches Organ, eine entscheidende Bedeutung beigemessen werden muss. So wirkt die durch Muskelaktivität hervorgerufene Freisetzung sogenannter Myokine, viszeralem Fett entgegen. Myokine hemmen entzündungsfördernde Zytokine (Knudsen u. Pedersen 2015) und können günstige neuroplastische Prozesse in Gang setzen, wie sie im Bereich der Depression bereits bekannt sind und nun auch im Zusammenhang mit chronischem Schmerz diskutiert werden (Price u. Inyang 2015; Leung et al. 2016).

Ballaststoffreiche Ernährung mit niedriger glykämischer Last sorgt über Magenfüllung und niedrige Blutzuckerwirkung für ein nachhaltiges Sättigungsgefühl. Die nicht verdaulichen Faserstoffe dienen aber auch als sogenannte Prebiotika einigen essentiellen Bakterienstämmen des menschlichen Mikrobioms als Energiequelle (Chung et al. 2016). Diese beeinflussen z. B. die Nahrungsresorption durch den Darm, oder besitzen immunmodulierende Kompetenz. Über die „gut-brain axis“kann das Mikrobiom mit dem menschlichen ZNS kommunizieren. Über diese Verbindung scheint es auch einen hemmenden Einfluss auf zentral inflammatorische Prozesse ausüben zu können, wie sie u. a. bei Depression, Demenz, aber auch chronischen Schmerzen diskutiert werden (Rea et al. 2016).

Richtig dosierte körperliche Aktivierung und individuell angepasste Energiezufuhr sollten die grundlegenden Säulen eines multimodalen Therapiekonzeptes bilden. Erst darauf basierend, können spezifische Therapien ihre volle Wirksamkeit entfalten.

Die Autoren, Ulrich Hamberger und Martina M Hinner (New Jersey, USA) entwickelten als Team Uhma International das vorgestellte Konzept (Hamberger u. Hinner 2014).

\section{Literatur}

Buyken AE, Goletzke J, Joslowski G, Felbick A, Cheng G, Herder C, Brand-Miller JC (2014) Association between carbohydrate quality and inflammatory markers: systematic review of observational and interventional studies. In: The American journal of clinical nutrition 99(4):813-833. DOI: 10.3945/ajcn.113.074252.

Chung WSF, Walker AW, Louis P, Parkhill J, Vermeiren J, Bosscher D et al (2016) Modulation of the human gut microbiota by dietary fibres occurs at the species level. In: BMC biology 14:3. DOI: 10.1186/s12915-015-0224-3.

Dunbar JA, Reddy P, Davis-Lameloise N, Philpot B, Laatikainen T, Kilkkinen A et al (2008) Depression: an important comorbidity with metabolic syndrome in a general population. Diabetes care 31(12):2368-2373.

Ghazarian M, Luck H, Revelo XS, Winer S, Winer DA (2015) Immunopathology of Adipose Tissue during Metabolic Syndrome. In: Turk patoloji dergisi 31 (Suppl 1):172180. DOI: 10.5146/tjpath.2015.01323.

Hamberger U, Hinner MM (2014) Die Macht der Muskeln, S.15.

Knudsen SH, Pedersen BK (2015) Targeting Inflammation Through a Physical Active Lifestyle and Pharmaceuticals for the Treatment of Type 2 Diabetes. In: Current diabetes reports 15(10):82. DOI: 10.1007/s11892-015-0642-1.

Leung L, Cahill CM (2010) TNF-alpha and neuropathic pain - a review. In: Journal of neuroinflammation 7:27. DOI: 10.1186/1742-2094-7-27.

Mayer EA, Knight R, Mazmanian SK, Cryan JF, Tillisch K (2014) Gut microbes and the brain: paradigm shift in neuroscience. In: The Journal of neuroscience : the official journal of the Society for Neuroscience 34(46):15490-15496. DOI: 10.1523/JNEUROSCl.3299-14.2014.

Morris G, Berk M, Carvalho A, Caso JR, Sanz Y, Walder K, Maes M (2016) The Role of the Microbial Metabolites Including Tryptophan Catabolites and Short Chain Fatty Acids in the Pathophysiology of Immune-Inflammatory and Neuroimmune Disease. In: Molecular neurobiology. DOI: 10.1007/s12035-016-0004-2.

Pedersen BK (2011) Muscles and their myokines. In: The Journal of experimental biology 214 (Pt 2):337-346. DOI: 10.1242/jeb.048074.

Price TJ, Inyang KE (2015) Commonalities between pain and memory mechanisms and their meaning for understanding chronic pain. In: Progress in molecular biology and translational science 131:409-434. DOI: 10.1016/bs.pmbts.2014.11.010.

Wensveen FM, Valentic S, Sestan M, Turk Wensveen T, Polic B (2015) The „Big Bang“ in obese fat: Events initiating obesity-induced adipose tissue inflammation. In: European journal of immunology 45 (9):2446-2456. DOI: 10.1002/eji.201545502.

\section{P07.03 - Fehldiagnose CRPS - L-Dopa-responsive schmerzhafte Dystonie nach Sprunggelenksfraktur}

S. Wunder', M. Kitzrow', L. Eitner ${ }^{3}$, C. Maier ${ }^{4}$

'BG Universitätsklinikum Bergmannsheil Bochum, Abteilung für Schmerzmedizin, Bochum, Deutschland, ${ }^{2}$ Klinikum Bergmannsheil, Neurologische Klinik, Bochum, Deutschland, ${ }^{3}$ BG Universitätsklinikum Bergmannsheil Bochum, Abteilung für Schmerzmedizin, Bochum, Deutschland, ${ }^{4}$ Berufsgenossenschaftliches Universitätsklinikum Bergmannsheil GmbH, Bochum, Abteilung für Schmerzmedizin, Bochum, Deutschland

CRPS ist heute eine nicht seltene Fehldiagnose mit daraus resultierender Therapieresistenz. Wir berichten über eine 44-jährigen Patientin ohne neurologische Vorerkrankung, die nach einer Teilruptur der Sehne des M. tibialis posterior wegen unzureichender Schmerzkontrolle eine Sehnenraffung mit Calcaneus-Teilosteotomie erfuhr. Postoperativ entwickelte sich eine mit hochdosierten Opioiden nicht beherrschbare Belastungssinsuffizenz mit schmerzhafter kontrakter Supinationsfehlstellung des Fußes. Nachdem Bisphosphonate, orale und intravenöse Opioide, Ko-Analgeti$\mathrm{ka}$, periphere und rückenmarksnahe Blockaden erfolglos blieben, wurde 
die Patientin in unsere Schmerzklinik mit der Verdachtsdiagnose eines CRPS gemäß den Budapest Kriterien (MRT und CT ohne path. Befund) überwiesen. Bei inzwischen bereits 3-monatiger Rollstuhlpflichtigkeit war die 3-Phasen-Skelettszintigrafie. unauffällig, eine Nervenläsion wurde mittels Quantitativ Sensorischer Testung ausgeschlossen. In Narkose war eine Neutralstellung möglich, obwohl die dystone Kontraktur sich nur partiell löste.

Nach L-Dopa-Gabe (200 mg) konnte die Patientin den Fuß erstmals fast in Neutralstellung aufsetzen. Unter der sich anschließenden oralen Erhaltungstherapie mit $200 \mathrm{mg}$ L-Dopa/Tag war eine vollständige Mobilisierung und Wiederherstellung der Arbeitsfähigkeit möglich. Alle Analgetika konnten abgesetzt werden (Follow-up: 6 Monate). Umfangreiche genetische und Stoffwechsel-Untersuchungen aus Serum und Liquor erlauben keine Zuordnung des Fall zu einem bekannten hereditären Dystonie-Syndrom (z. B. Segawa Syndrom)

Der Fall unterstreicht erneut, dass ein CRPS nicht eine Ausschlussdiagnose sein darf, sondern auf positiven Kriterien z. B. der 3-Phasen-Szintigrafie [1] beruhen sollte. Fokale Dystonien können Teilsymptom eines CRPS sein [2], aber auch eine davon unabhängige posttraumatische Fehlregulation wie in diesem Fall.

\section{Literatur}

1. Wüppenhorst et al (2010) Sensitivity and specificity of 3-phase bone scintigraphy in the diagnosis of complex regional pain syndrome of the upper extremity. Clin Pain 26:182-189

2. Van Hilten et al (2000) Intrathecal Baclofen for the treatment of dystonia in patients with reflex sympathetic dystrophy. N Engl J Med 343:625-630

3. Wijemanne S, Jankovic J (2015) Dopa-responsive dystonia-clinical and genetic heterogeneity. Nat Rev Neurol11:414-424

\section{P07.04 - Existieren geografische Unterschiede für die Sensitivität bei der Untersuchung thermischer Schwellen zwischen einer arktischen und einer mitteleuropäischen Population?}

\section{H. Nothnagel', C. Puta' ', H. Gabriel', T. Weiss', F. Musial ${ }^{3}$ \\ 'Friedrich-Schiller-Universität Jena, Lehrstuhl für Sportmedizin, Jena, Deutschland, ${ }^{2}$ Institut für Psychologie, FSU Jena, Biologische und Klinische Psychologie, Jena, Deutschland, ${ }^{3}$ National Research Centre for Complementary and Alternative Medicine (NAFKAM), UiT, The Artic University of Norway, Tromsø, Norwegen}

Hintergrund und Ziel der Studie: Für die Quantitative sensorische Testung (QST) existieren geschlechts- und altersspezifische Referenzwerte für verschiedene Messareale (Gesicht, Hand, Fuß und Rumpf) [1,2]. Darüber hinaus sind auch kulturelle und ethnische Einflüsse auf die Schmerzwahrnehmung und Schmerzschwellen bekannt $[3,4,5]$. Geografische und klimatische Einflüsse wurden hingegen weniger untersucht.

Zentrale Zielstellung der vorliegenden Studie war der Vergleich der thermischen QST-Parameter zwischen einer arktischen und einer mitteleuropäischen Stichprobe.

Methode: Es wurden insgesamt 28 gesunde Probanden untersucht: 9 norwegische Probanden aus Tromsø (Norwegen, 2 männlich, 7 weiblich, Alter: 42,9 $\pm 7,5$ Jahre, MW \pm SD) und 19 deutsche Probanden aus Jena (Deutschland, 8 männlich, 11 weiblich, Alter: 46,1 $\pm 13,6$ Jahre, MW \pm SD). Die Detektionsschwellen für Kälte und Wärme (CDT, WDT) sowie die Schmerzschwellen für Kälte und Hitze (CPT, HPT) wurden entsprechend dem standardisierten QST-Protokoll des Deutschen Forschungsverbund Neuropathischer Schmerz (DFNS) [6] von zwei Untersucherinnen (H.N. und B. J.D.) an der dominanten Hand (dorsum) gemessen.

Zur Auswertung der Studienergebnisse wurden die Parameter CDT und WDT $\log 10$-transformiert (sekundäre Normalverteilung). Zur statistischen Analyse der Schwellenunterschiede wurden die thermischen QSTParameter beider Stichproben mittels T-Test bzw. Mann-Whitney-U-Test untersucht.

Ergebnisse: Die statistische Analyse zeigte für die thermischen Schmerzschwellen signifikante Unterschiede zwischen der arktischen und der mitteleuropäischen Population; CPT: Tromsø (MW \pm SD: 17,93 $\pm 11,05)$, Jena $(\mathrm{MW} \pm \mathrm{SD}: 6,73 \pm 7,55), p=0,006$; HPT: Tromsø (MW \pm SD: 42,94 $\pm 3,84)$,
Jena (MW \pm SD: $46,12 \pm 2,89$ ), $p=0,022$. Für die thermischen Detektionsschwellen für Kälte (CDTlog, $p=0,85$ ) und Wärme (WDTlog, $p=0,97$ ) wurden keine signifikanten Unterschiede zwischen der arktischen und der mitteleuropäischen Stichprobe gefunden.

Diskussion \& Schlussfolgerungen: Die Ergebnisse der Studie zeigen einen signifikanten Unterschied der thermischen Schmerzschwellen (CPT, HPT) an der Hand zwischen der arktischen und der mitteleuropäischen Stichprobe. Die erhöhten Schmerzschwellen der nord-norwegischen Population deuten darauf hin, dass die Norweger, bezogen auf Kälte- und Hitzeschmerz, offensichtlich sensitiver reagieren. Es ist anzunehmen, dass dieser Effekt mit den klimatischen und ggf. geografischen Bedingungen (Lage zum Äquator bzgl. der Lichtexploration) assoziiert ist und möglicherweise eine Anpassungsleistung an die klimatischen Gegebenheiten darstellt. Untersuchungen in vergleichbaren geografischen Lagen sollten diesen Aspekt der veränderten Thermosensitivität bei klinischen Fragestellungen berücksichtigen.

\section{Literatur}

1. Magerl et al (2010) Reference data for quantitative sensory testing (QST): Refined stratification for age and a novel method for statistical comparison of group data. Pain 151(3):598-605.

2. Pfau et al (2014) Quantitative sensory testing in the German Research Network on Neuropathic Pain (DFNS): reference data for trunk and application in patients with chronic postherpetic neuralgia. Pain 155(5):1002-1015.

3. Dawson et al (2009) Comparison of pain thresholds and pain tolerance levels between Middle Easterners and Swedes and between genders. Journal of Oral Rehabilitation 36(4):271-278.

4. Edwards et al (2001) Race, ethnicity and pain. Pain 94(2):133-137.

5. Edwards et al (1999) Ethnic Differences in Thermal Pain Responses. Psychosomatic Medicine 61(3):346-354.

6. Rolke et al (2006) Quantitative sensory testing in the German Research Network on Neuropathic Pain (DFNS): Standardized protocol and reference values. Pain 123(3):231-43.

\section{P07.05 - Sind wir zu spät? - Erfahrungen mit dem stationären interdisziplinären Schmerzassessment}

\section{Mothes-Lasch, E. Sens, B. Otto, J. Lutz}

\section{Zentralklinik Bad Berka $\mathrm{GmbH}$, Zentrum für Interdisziplinäre} Schmerztherapie, Bad Berka, Deutschland

Die NVL Kreuzschmerz empfiehlt bereits nach 6 bzw. 12 Wochen bestehender Schmerzen ein Schmerzassessment zur Bahnung einer optimalen und frühzeitigen Behandlung verschiedener Schmerzsyndrome. Ein wesentliches Ziel ist dabei, Chronifizierung zu verhindern und Folgekosten zu minimieren. Das Zentrum für interdisziplinäre Schmerztherapie in Bad Berka (ZIST) hat von 2008 bis 20152704 stationäre interdisziplinäre Schmerzassessments (ISA) gemäß dem OPS-Code 1-910 „Multidisziplinäre algesiologische Diagnostik“ und den Empfehlungen der Ad-HocKomission der DGSS durchgeführt. Die Daten der Patienten wurden deskriptiv und mittels parametrischer Tests ausgewertet.

Die Patienten des ZIST waren überwiegend hochchronifiziert (MPSS III 90,6\%) und hochbeeinträchtigt (von Korff IV 74,1\%). Sie wiesen bezüglich des Schmerzes häufig eine langjährige Krankengeschichte (MW 13,1 Jahre) und eine hohe Anzahl in Anspruch genommener medizinischer Leistungen auf. Ein Großteil war entweder arbeitsunfähig (21,6\%) oder bereits berentet $(53,9 \%)$. Viele Patienten waren medikamentös mit Opioiden versorgt $(43,7 \%)$, wobei den meisten dieser Patienten bei unzureichender Schmerzlinderung und bestehender Nebenwirkungen am Ende des ISAs ein Opioidentzug empfohlen wurde (88,7\%). Die psychometrischen Fragebogenwerte zeigten im Hinblick auf Lebensqualität, Depressivität, Ängstlichkeit und subjektive Stressbelastung erhöhte Werte und somit eine hohe psychosoziale Gesamtbelastung.

Als Ergebnis des ISAs wurden den Patienten eine ambulante Weiterbehandlung (19,5\%), eine andere stationäre Behandlung (20,4\%) oder die Teilnahme an einer stationären multimodalen Schmerztherapie (MMST, $60,1 \%$ ) empfohlen. Bei 42,4\% der Patienten, die sich in dem ISA vorstellten, ist mittlerweile eine MMST am ZIST erfolgt. Bemerkenswert ist dabei eine signifikante Verbesserung der Mittelwerte der psychometrischen 
Kennwerte schon zu Therapiebeginn im Vergleich zum ISA in Bezug auf die Lebensqualität, Depressivität und subjektive Stressbelastung mit mittleren Effektstärken und Verbesserungen in Bezug auf die Ängstlichkeit mit geringen Effektstärken. Diese Ergebnisse deuten an, dass durch das ISA nicht nur innerhalb kurzer Zeit eine umfassende Diagnostik erfolgen und eine individuelle Therapieempfehlung erarbeitet werden können, sondern auch die psychosoziale Belastung bereits beeinflusst wird.

Die Analyse der vorliegenden Stichprobe zeigt in Verbindung mit einer langen Schmerzdauer, einer hohen psychischen Gesamtbelastung und einer inadäquaten medikamentösen Versorgung, dass ein in der individuellen Krankengeschichte früheres ISA, wie von der NVL gefordert wird, medizinisch und ökonomisch sinnvoll erscheint. Dennoch sind die Empfehlungen des ISAs für Patienten aller Chronifizierungs- und Beeinträchtigungsgrade ergebnisoffen. Darüber hinaus scheint das ISA die Motivation für einen aktivierenden Therapieansatz zu stärken, was in weiteren Studien untersucht werden sollte.

\section{P07.06 - Schmerzassessment in der vollstationären Altenhilfe - S3 Leitlinie}

I. Gnass' ${ }^{1}$, E. Berkemer', K. Bündel ${ }^{3}$, C. Drebenstedt', J. Dreyer ${ }^{5}$, T. Fischer ${ }^{6}$, P. Higman $^{7}$, K. Kopke ${ }^{8}$, M. Laekeman ${ }^{9}$, C. Leonhardt ${ }^{10}$, A. Lukas ${ }^{11}$, M. Schuler ${ }^{12}$, E. Sirsch $^{13}$

'Paracelsus Medizinische Privatuniversität, Salzburg, Österreich, ${ }^{2}$ Hochschule Ludwigshafen, Ludwigshafen, Deutschland, ${ }^{3}$ Deutsche Gesellschaft für Allgemeinmedizin und Familienmedizin (DEGAM), Berlin, Deutschland, ${ }^{4}$ St. Marien-Hospital, Friesoythe, Deutschland, ${ }^{5}$ Deutsches Zentrum für Neurodegenerative Erkrankungen (DZNE), Witten, Deutschland, ${ }^{6}$ Evangelische Hochschule für soziale Arbeit, Dresden, Deutschland, ${ }^{7}$ Deutscher Verband der Ergotherapeuten (DVE) e.V., Karlsbad-Itter, Deutschland, ${ }^{8}$ Hochschule für angewandte Wissenschaften Hamburg, Hamburg, Deutschland, ' Universität Witten/ Herdecke, Bochum, Deutschland, ${ }^{10}$ Philipps-Universität Marburg, Marburg, Deutschland, "'Malteser Krankenhaus, Bonn/Rhein-Sieg, Deutschland, ${ }^{12}$ Diakoniekrankenhaus, Mannheim, Deutschland, ${ }^{13}$ PhilosophischTheologische Hochschule, Vallendar, Deutschland

Hintergrund: Schmerz stellt ein häufiges Problem in der älteren Bevölkerung dar. Bis zu $80 \%$ der Bewohnerinnen in Einrichtungen der stationären Altenhilfe leiden darunter (Osterbrink, et al., 2012; Lukas, et al., 2015). Bis zur Hälfte aller in stationären Einrichtungen der Altenhilfe lebenden Personen erhalten keine, bzw. eine nicht adäquate schmerztherapeutische Versorgung, obwohl sie unter relevanten Schmerzen leiden (Pickering, et al., 2006). Vor diesem Hintergrund haben pflegerische, ärztliche, psycho-, physio- und ergotherapeutische Experten und Patientenvertreter unter Federführung des Arbeitskreises „Schmerz und Alter“ der Deutschen Schmerzgesellschaft eine Leitlinie zum „Schmerzassessment bei alten Menschen in der vollstationären Altenhilfe" erstellt.

Der Entwicklungsprozess fand mit Einbindung von 39 Fachgesellschaften statt.

Methodik: Die bei der Arbeitsgemeinschaft Wissenschaftlicher Medizinischer Fachgesellschaften (AWMF) angemeldete Leitlinie wurde in Steuer-, Arbeits- und Konsensgruppen koordiniert. Die Methodik zur Entwicklung der Leitlinie der Stufe 3 folgt dem Regelwerk der AWMF. Zuerst wurden klinische Fragen analog zum Versorgungsprozess entwickelt. Mit vordefinierten Suchbegriffen erfolgt die systematische Suche nach Quellleitlinien, die einer methodischen und inhaltlichen Analyse unterzogen wurden. Im nächsten Schritt wurden neun Kernfragen für eine systematische Literaturrecherche nach Primärquellen identifiziert. Die beiden Suchen fanden u. a. in den Datenbanken Cochrane Library, Medline, National Guideline Clearinghouse statt. Zur Konsensfindung wurden Delphiverfahren und Konsensuskonferenzen angewendet.

Ergebnisse: Die eingeschlossenen 12 Quellleitlinien und 72 Primärquellen zeigten bei der methodischen Beurteilung eher geringe Evidenzgrade. Die Leitliniengruppe hat trotz geringer Evidenz, im Kontext der klinischen Versorgungsrelevanz entlang der Versorgungspraxis Empfehlungen formuliert, die eine Verbesserung im Schmerzassessment bei älteren Bewoh- nerinnen fokussieren. Letztendlich wurden zwei Empfehlungen zum Deutungskontext Schmerz, vier zu kognitiver Beeinträchtigung und Schmerz, 18 zum Schmerzscreening, 25 zum Schmerzassessment und 13 zur Verlaufserfassung von Schmerz formuliert. Die Vielzahl der Empfehlungen zeigt detailliert und fokussiert Aspekte zum Vorgehen bei chronischem und akutem Schmerz auf, die auch die Auskunftsfähigkeit der Bewohnerinnen berücksichtigt. In einer finalen Konsensuskonferenz im Februar 2016 wurden alle Empfehlungen konsentiert.

Schlussfolgerungen: Mit der Leitlinie sind wesentliche klinische Fragestellungen zum Schmerzassessment bei Bewohnern und Bewohnerinnen in der vollstationären Altenhilfe beantwortet. Es zeigt sich ein genereller Forschungsbedarf, z. B. zur Trennschärfe zwischen Screening und Assessment, dem Einsatz von Schmerztagebüchern sowie der Überprüfbarkeit der Empfehlungen nach Implementierung.

\section{P07.07 - Ein Kunstprojekt über den chronischen Schmerz: Eine qualitative Studie der Erfahrungen von einem Leben mit Schmerz im Zusammenhang mit der Familie von erwachsenen Patienten}

P. Walton

University of Cumbria, Faculty of Education, Arts \& Business, Carlisle, UK

Der Schmerz an sich, ist eine subjektive und abstrakte Erfahrung, die wir nur schwer mit anderen teilen können. Ein Leben mit chronischen Schmerzen, kann hochgradige Auswirkungen auf den Patienten, so wie seine Familie und unmittelbares Umfeld haben. Oftmals beruhen Studien auf den Erfahrungen von Patienten, während die Erfahrungen der unmittelbaren Familie außer Acht gelassen werden. Diese Studie ist ein Versuch diese ,Lücke' zu schließen und mittels Fotografie die Erfahrungen von Patienten und deren Familie gleichberechtigt visuell darzustellen.

Die Studie beruht auf Aussagen von vier Familien, jeweils mit einem erkranktem Elternteil, die getrennt voneinander über ihr Leben mit dem Schmerz berichtet haben. Ausgewertet wurden diese Gespräche mit einem interpretativ-phänomenologischen Ansatz, um sich ganz auf die Lebenserfahrungen der Familien zu konzentrieren.

Wichtig ist an dieser Stelle zu erwähnen, dass ich selbst chronischer Schmerzpatient bin. Dies verschaffte mir ein besonders gutes Verständnis der Problematik auf einer emotionalen Ebene, statt die Erfahrungen rein klinisch zu betrachten.

Das Ziel dieses Projekts war, die Erfahrungen der Familien zu validieren und diese in eine visuelle Sprache zu übersetzen. Bestandteil davon war, eine unsichtbare und schwer greifbare Krankheit sichtbar zu machen. Für den Betrachter erfordert dies, die Auseinandersetzung mit dem Thema auf einer emotionalen statt einer intellektuellen Ebene, und bietet die Möglichkeit, die Erfahrungen auf einer nicht-medizinischen Ebene zu kommunizieren. Dieser Ansatz kann Patienten und deren Familien die Möglichkeit gewähren, Kommunikationsschwierigkeiten untereinander oder mit Außenstehenden wie Freunden, Arbeitskollegen oder auch Ärzten zu überwinden.

„Artistic works in music, dance, and visual arts are a media for expressions of meaning and the ,voice to suffering which is inadequately expressed in words. Artistic expressions are the conduit to personal and universal experiences of suffering and, in return, provide relief and give meaning to that experience." [1]

\section{Literatur}

1. Davis AA, Davis MP (2010) Art in medicine and the art of grief. Prog Palliat Care 18(5):266-269 


\section{P08 - Neuropathischer Schmerz II}

\section{P08.01 - Die Dynamik des somatosensorischen Systems bei Patienten mit postherpetischer Neuralgie}

\section{J. Gierthmühlen, O. Esau, J. Hellriegel, R. Baron}

Universitätsklinikum Schleswig-Holstein, Campus Kiel Klinik für Neurologie, Kiel, Deutschland

Einleitung: Bei der postherpetischen Neuralgie (PHN) können verschiedene Patiententypen hinsichtlich der peripheren Nozizeptorfunktion unterschieden werden. Untersuchungen zur möglichen Veränderungen der Somatosensorik im Verlauf der PHN sind selten. Ziel war es daher, somatosensorische Symptome im Verlauf der Erkrankung mit besonderem Augenmerk auf die verschiedenen Subtypen der PHN zu untersuchen. Methodik: 20 PHN-Patienten (7 Männer, 13 Frauen, Alter 67 $\pm 9,6$ Jahre (40-77 Jahre); Erkrankungsdauer 18,1 \pm 26 Monate (1 -97 Monate)) wurden bei Erstvorstellung und 31,6 $\pm 23,8$ Monate später untersucht und eine QST (Protokoll des DFNS) durchgeführt. Die Auswertung der QST erfolgte nach den Richtlinien des DFNS. Der Wilcoxon- und der U-Test wurden für den Vergleich innerhalb bzw. zwischen den Gruppen verwendet, zur Analyse der Häufigkeiten abnormaler QST-Werte wurde der Chi-Quadrat-Test verwendet. Lineare Zusammenhänge wurden mit dem Pearson - Korrelationskoeffizienten beurteilt. $\mathrm{P}<0,05$ wurde als statistisch signifikant angesehen.

Ergebnisse: 14 (70\%) PHN-Patienten zeigten eine beeinträchtigte (iPHN) und $6(30 \%)$ eine erhaltene (pPHN) C-Faser-Funktion. iPHN zeigten bei der Ausgangsuntersuchung verglichen mit pPHN einen stärkeren Verlust von thermischer und mechanischer Detektion und schmerzhafter Hitzeempfindung auf der betroffenen Seite $(p<0,05)$. Die Gruppen unterschieden sich nicht in Alter, Erkrankungsdauer oder Schmerzintensität.

Beide Gruppen unterschieden sich nicht bezüglich Verbesserung der Schmerzintensität im Verlauf ( $-0,5 \pm 2,3$ vs $-1,7 \pm 2,6$ NRS, p n. s.), Schmerzintensität (5,0 +/- 3,9 vs 4,2 +/- 2,8 NRS, p n. s.) oder Erkrankungsdauer $(43,5 \pm 3,8$ vs $64,2 \pm 63$ Monate, $p$ n. s.) bei der Folgeuntersuchung.

Die thermische und mechanische Detektion sowie DMA verbesserte sich bei iPHN im Vergleich zu pPHN (CDT: $0,8 \pm 1,4$ vs $-1,2 \pm 1,2$, WDT: $1,8 \pm 2,1$ vs $-0,7 \pm 1,7$; TSL: $1,0 \pm 1,1$ vs $-0,5 \pm 1,3$; MDT: $2,5 \pm 7,8$ vs $-1,9 \pm 3,1$ DMA: $-5,6 \pm 12,2$ vs $11,4 \pm 17,8, p<0,05$ für alle). Die Verbesserung des Detektionsverlustes war unabhängig von der Änderung der Schmerzintensität. PPHN zeigte dagegen einen Anstieg der mechanischen Schmerzempfindlichkeit (MPS: $-0,38 \pm 2,2$ vs $1,4 \pm 2,5, p<0,05$ ) und eine Tendenz zu stärkerem Detektionsverlust bei der Folgeuntersuchung.

Schlussfolgerung: Die Ergebnisse zeigen, dass (A) die Schmerzverbesserung unabhängig vom Subtyp der PHN ist, aber (B) Patienten mit pPHN stärker für die Entwicklung einer zentralen Sensibilisierung prädisponiert sind. (C) Eine iPHN kann sich selbst bei chronischen Fällen (> $1 \mathrm{Jahr}$ ) verbessern; wobei hier eine schmerz-induzierte Hypoästhesie unwahrscheinlich ist.

Insgesamt zeigen die Ergebnisse die Dynamik des somatosensorischen Systems und können eine mechanismen-basierte Behandlung verbessern. So können Patienten mit pPHN von zentral wirkenden Analgetika und iPHN auch von topischen Behandlungen profitieren. Weitere Studien sind notwendig, um diesen Aspekt zu behandeln.

\section{P08.02 - Capsaicin $8 \%$ cutanes Pflaster zur Behandlung von Phantom- und Stumpfschmerzen - Auswertung einer retrospektiven Fallserie nach Majoramputation}

\section{B. Schlisio}

Universität Tübingen, Klinik für Anästhesiologie und Intensivmedizin, Tübingen, Deutschland

Die Behandlung von Phantom- und Stumpfschmerzen gehört zu den am schwierigsten zu behandelnden Schmerzentitäten, weil die medikamentöse Behandlung nicht wirksam oder nebenwirkungsbehaftet ist. Die Behandlung von neuropathischen Schmerzen durch Capsaicin 8\% cutanes
Pflaster ist ein etabliertes Therapieverfahren, welches unabhängig von der Genese der Neuropathie zugelassen und eingesetzt wird.

Methoden: Unserer Fallserie dokumentiert retrospektiv und systematisch das Therapieergebnis nach der Behandlung durch Capsaicin $8 \%$. Folgende Kriterien wurden zur Einstufung als Responder bzw. Nichtresponder herangezogen.

- Schmerzveränderung in Ruhe und Belastung

- Schmerzintensität mit und ohne Prothese und Veränderungen bezüglich der

- Tragedauer der Prothese

- Veränderung der Lebensqualität (dichotome Fragestellung)

- Verbesserung der Schlafqualität

- Reduktionsmöglichkeit der Schmerzmedikation

Insgesamt konnten 15 Patienten in dieser Serie ausgewertet werden.

Ergebnisse: Alle Patienten erfüllten die typische Phänomenologie neuropathischer Schmerzen. Zwei Drittel der Patienten konnten als Therapieresponder eingestuft werden. Die Behandlung wurde den Respondern zur Wiederholung angeboten. Nebenwirkungen waren lokale Rötungen, die sich im Verlauf wieder zurückbildeten.

Die gemachten Ergebnisse werden mit einer aktuellen Literaturanalyse zu den Behandlungsergebnissen mit Capsaicin $8 \%$ diskutiert.

Schlussfolgerung: Capsaicin $8 \%$ kann als Option für den therapieresistenten Phantom- und Stumpfschmerz versucht werden. Es ist keine Off- Label Behandlung. Das Nebenwirkungsrisiko ist gering und nicht schwerwiegend. Randomisiert kontrollierte Studien zu dieser Indikation von Capsaicin $8 \%$ cutanes Pflaster fehlen. Die Auswertung einer Subgruppe von 21 Patienten mit Postamputationsschmerzen zeigte im Rahmen der Queppstudie [1] ähnliche Ergebnisse. Der Empfehlungsgrad zur Anwendung von Capsaicin $8 \%$ cutanes Pflaster für diese Indikation entspricht aufgrund der der kleinen Fallzahl und der niedrigen Qualität der Studien einer Expertenmeinung.

Der Beitrag wurde unterstützt von Fa. Astellas Pharma GmbH, München: Ausrichtung des Expertentreffens („Fokusgruppe Qutenza ${ }^{\mathrm{Th}}$ 8\%“) am 15.4.2016 (organisiert von Herrn Paul Kergel, Fa. Astellas Pharma GmbH)

\section{Literatur}

1. Kern K-U, Baust H, Hofmann W, Holzmüller R, Maihöfner C, Heskamp M-L (2014)

Das 8\%ige Capsaicin-Pflaster bei Phantomschmerz. Schmerz 28:374-383

\section{P08.03 - Topisches Ambroxol zur Therapie des CRPS - Eine neue Option?}

\section{K. Kern', S. Schneider', P. Bialas ${ }^{3}$, H. Gockel', C. Maihöfner}

'Institut für Schmerzmedizin/Schmerzpraxis Wiesbaden, Wiesbaden, Deutschland, ${ }^{2}$ Schmerz- u. Palliativzentrum, Wiesbaden, Deutschland, ${ }^{3}$ Klinik f. Anästhesiologie, Universität Homburg Saar, Homburg, Deutschland, ${ }^{4}$ Algesiologikum, Interdisziplinäres Zentrum f. Schmerztherapie, Vilsbiburg, Deutschland, ${ }^{5}$ Klinikum Fürth, Chefarzt der Neurologischen Klinik, Fürth, Deutschland

Einleitung: Kürzlich konnten wir erstmals die topische Behandlung neuropathischer Schmerzen mit Ambroxol 20\% Creme in einer Fallserie beschreiben. Die Tatsache, dass die Substanz auch entzündungshemmend wirkt, oxidativen Stress reduziert, Radikalfänger ist und Einfluss auf vasomotorische Prozesse nimmt, macht sie interessant als Behandlungsoption für das komplexe regionale Schmerzsyndrom (CRPS).

Methode: 7 Patienten wurden (meist 2x tgl.) mit topischem Ambroxol $20 \%$ (entsprechend d. Rezeptur der Erstpublikation) von den Autoren behandelt. Alle Behandlungen erfolgten als individuelle Therapieversuche nach vorheriger Aufklärung und Einwilligung und als Ergänzung zum ortsüblichen Therapieschema. Alle Patienten erfüllten die Budapest-Kriterien zur Diagnose eines CRPS. Die Behandlungsverläufe wurden deskriptiv dokumentiert und später exemplarisch zusammengetragen. Bei 2 Patienten erfolgten zusätzlich Messungen der Hauttemperatur im Seitenvergleich und Messungen des Finger-Hohlhand-Abstandes.

Ergebnisse: Die Erkrankungsdauer lag zwischen 4 Wochen und 11 Monaten. Viermal war die Hand betroffen, 1x der Fuß und 1x Unterarm/Handgelenk. In je 5 Fällen lag eine dynamische Allodynie und mechanische Hy- 
peralgesie vor, $1 \mathrm{x}$ nicht und $1 \mathrm{x}$ nicht mehr. Von 5 Patienten mit Ödem war dies klinisch bei 2 Betroffenen nach 1 Tag reduziert, in einem weiteren Fall bereits nach $30 \mathrm{~min}$, in einem anderen nach $2 \mathrm{~h}$. Die 3 Patienten mit Rötung erlebten in der gleichen Zeit auch hier eine Reduktion, in einem Fall bereits nach $3 \mathrm{~h}$. Veränderungen der Hauttemperatur ergaben sich bei den beiden gemessenen Patienten nicht. Von 6 Patienten mit funktioneller Einschränkung zeigten 2 eine messbare Verbesserung des Finger- HohlhandAbstandes nach spätestens 24 h, 3 Patienten eine subjektive Verbesserung in der 1 . Woche. Ein Patient reagierte bei keinem der oben genannten Kriterien positiv. Schmerzreduktionen in Ruhe beschrieben 6 der 7 Patienten, 2 in den ersten beiden Stunden, 4 nach wenigen Tagen. Die deutlichsten Schmerzreduktionen waren: bei Belastung von NRS 8 auf NRS 5 in der 1 . Stunde, in Ruhe von NRS 7 auf NRS 0 nach 1 Woche. Ein Patient mit 2monatiger Erkrankungsdauer, einem Belastungsschmerz von NRS 6-8 und erheblicher Funktionseinschränkungen im Ellenbogengelenk war nach 4 Wochen schmerzfrei und ohne funktionelle Einschränkung.

Diskussion: Eine Natriumkanalblockade (bes. v. $\mathrm{Na}_{\mathrm{v}} 1.8$ ), antiinflammatorische Eigenschaften und die Reduktion oxidativen Stresses machen topisches Ambroxol $20 \%$ zu einer interessanten Option zur Behandlung des CRPS. Besonders die frühen Veränderungen von Schmerz, Ödem, Rötung und Funktionsverbesserungen legen eine Wirkung der Substanz bei den von uns behandelten Patienten nahe. Dennoch erhielten alle Patienten parallel ja eine ortsübliche Standardtherapie, so dass die Behandlungserfolge nicht alleine oder gesichert Ambroxol zugeordnet werden können. Wir betrachten den Behandlungsansatz aber als interessante Option, die systematisch weiter untersucht werden sollte.

\section{P08.04 - Nachweis der positiven Wirkung von nicht-visuellen Effekten einer Lichtexposition auf Somatosensorik und Nozizeption bei CRPS}

C. Maihöfner ${ }^{1}$

${ }^{1}$ Klinikum Fürth, Chefarzt der Neurologischen Klinik, Fürth, Deutschland

Fragestellung: Tierexperimentell konnte nachgewiesen werden, dass spezifische Ionenkanäle (vor allem TRPA1) des nozizeptiven Systems nachhaltig durch die Exposition mit blauem Licht moduliert werden können (1). Durch Nachweis der Wirksamkeit von nicht-visuellen Effekten einer Lichtexposition auf Somatosensorik und Nozizeption beim Menschen könnte der Einsatz einer Lichttherapie bei Patienten mit Erkrankungen des somatosensorischen Systems, insbesondere neuropathischen Schmerzen, von großer Bedeutung sein.

Material und Methoden: Untersucht wurden bei Patienten mit einem Komplex-Regionalen Schmerzsyndrom (CRPS) nicht-visuelle Effekte einer Lichtexposition auf Somatosensorik und Nozizeption. Die Patienten wurden für eine Stunde mittels einer Manschette entweder mit Wärme (Kontrolle) oder blauem Licht (Verum) an der erkrankten Hand exponiert. Vorher und nachher wurde eine Quantitativ Sensorische Testung (QST) durchgeführt. Zusätzlich wurden das subjektive Schmerzempfinden sowie thermische Effekte mittels Thermokamera erfasst.

Bisherige Ergebnisse: Bei allen 7 Patienten (3 Patienten mit warmen CRPS, 3 mit kaltem CRPS sowie 1 Patient ohne signifikante Temperaturdifferenz) zeigte sich nach einer Stunde Beleuchtungszeit ein signifikanter Temperaturanstieg im Beleuchtungsareal von mindestens $1,6^{\circ} \mathrm{C}$ und maximal $4,3^{\circ} \mathrm{C}$ (Mittelwert $2,86^{\circ} \mathrm{C}$ ). Die subjektive Schmerzintensität war bei den Patienten mit einem kalten CRPS deutlich vermindert. Bei Patienten mit warmen oder indifferenten CRPS zeigte sich keine Schmerzveränderung. Im QST zeigten sich signifikante Veränderungen thermischer und mechanischer Schwellen.

Schlussfolgerung: Bei kaltem CRPS scheint die angewandte Lichtexposition zu einer Verbesserung von CRPS- Symptomen zu führen.

\section{P08.05 - Reliability of the English version of the painDETECT questionnaire and its pain descriptors}

B. Tampin' ', B. Tampin'2, B. Tampin'3 , T. Bohne'2, M. Callan², M. Kvia', A. Melsom Myhre' ${ }^{2}$ E. Neoh ${ }^{2}$, C. Bharat ${ }^{4}$, H. Slater ${ }^{2}$

'Sir Charles Gairdner Hospital, Department of Physiotherapy, Nedlands, Australia, ${ }^{2}$ Curtin University, School of Physiotherapy and Exercise Science, Perth, Australia, ${ }^{3}$ Hochschule Osnabrück, University of Applied Sciences, Fakultät Wirtschafts-und Sozialwissenschaften, Osnabrück, Germany, ${ }^{4}$ University of Western Australia, M019 School of Maths and Stats" Crawley, Australia

Background: The painDETECT questionnaire (PD-Q) is used as a screening tool for the identification of neuropathic pain $(\mathrm{NeP})$. It consists of seven weighted pain descriptors and two pain specific behavioural pattern questions. While the English version of PD-Q has been widely used, its reliability has not been investigated.

The aim of this study was to determine the reliability of the English version of the PD-Q and its pain descriptors pre- and post-clinical consultation (T0-T1) and at one-week follow-up (T0-T2).

Methods: Patients $(\mathrm{N}=157)$ were recruited from a Neurosurgery Spinal Clinic and Pain Management Department. Intraclass Correlation Coefficients (ICC) were used to assess reliability of PD-Q total scores between T0-T1 and T0-T2; weighted kappa $(\kappa)$ was used to assess the agreement of PD-Q classifications (unlikely NeP, ambiguous, likely $\mathrm{NeP}$ ) and individual item descriptors between all time-points. To ensure stability of clinical pain, patients scoring $\leq 2$ or $\geq 6$ on the Patient Global Impression Scale (PGIC) at T2, were excluded from the T0-T2 analysis.

Results: Accounting for missing data and exclusions (change in PGIC score), data for 136 individuals (mean age (SD): 56,8 (15.2); 54\% male) were available, of which 129 patients were included in the T0-T1, and 69 patients in the T0-T2 comparison. There was almost perfect agreement between the PD-Q total scores at T0-T1 (ICC 0.911; $95 \%$ CI: 0.882-0.941) and substantial agreement at T0-T2 (ICC 0.792; 95\% CI: 0.703-0.880). PD-Q classifications demonstrated substantial agreement for T0-T1 (weighted $\kappa: 0.771 ; 95 \%$ CI: 0.683-0.858) and for T0-T2 (weighted $\kappa$ : 0.691; $95 \%$ CI: 0.553-0.830). For individual PD-Q items, weighted kappa values ranged between 0.63 (95\% CI: 0.53-0.73) and 0.83 (95\% CI: $0.74-$ $0.91)$ at T0-T1, indicating substantial to almost perfect agreement. At T0T2, Kappa scores varied between 0.30 (95\% CI: 0.13-0.46) and 0.85 (95\% CI: 0.70-0.99) such that there was almost perfect agreement for the item of pain radiation, heat/cold sensitivity showed substantial agreement and the items burning, tingling, light touch, numbness and pressure sensitivity demonstrated moderate agreement between measurements. The descriptors of pain attacks and pain course showed fair agreement between T0-T2. Discussion: Missing data was encountered in $13 \%$ of our cohort which may potentially lead to a false $\mathrm{NeP}$ classification on $\mathrm{PD}-\mathrm{Q}$. Changes in the mode of PD-Q administration (i.e. electronic version) may be worth considering. At all time-points measured, $42 \%$ to $56 \%$ of our patients drew multiple pain areas on the PD-Q body chart. While the presence of multiple pain areas seemed not to affect the reliability, it may question the validity of the PD-Q, as it remains unclear for which pain area patients answered the pain descriptors.

Conclusion: The English version of the PD-Q is reliable pre and post-consultation, and at one-week follow-up. Changes in the mode of PD-Q administration should be considered.

\section{P08.06 - Belastung durch lokalisierte, chronische postoperative neuropathische Schmerzen: Epidemiologische Daten aus einer laufenden multizentrischen Studie}

N. Damann', S. König' ', I. Bösl'

'Grünenthal GmbH, Clinical Development, Aachen, Deutschland

Einleitung: Lokalisierter, chronischer postoperativer neuropathischer Schmerz (PSNP, Post-Surgical Neuropathic Pain) nach Nervenfaserschädigung ist ein gravierendes klinisches Problem. Das postoperative Chronifizierungsrisiko beträgt $10-50 \%$, je nach Operations(OP)-Indikation $[1,2$, 
3]. Die Behandlung ist schwierig, da einzelne Medikamente oft nicht alle zugrundeliegenden Schmerzmechanismen abdecken. Der Bedarf an weiteren wirksameren Behandlungsmethoden ist hoch. Ziel dieser Untersuchung ist, einen genaueren Einblick in das Krankheitsbild PSNP zu erhalten.

Methodik: Demographische und anamnestische Daten aus einer laufenden multizentrischen Phase 3 Studie [4] in PSNP wurden analysiert. Patienten ab 18 Jahren wurden in die Studie eingeschlossen, wenn sie seit mindestens 3 Monaten nach einer OP unter mittelstarker bis schwerer PSNP litten. Alter, Geschlecht, PSNP-Dauer, Art der OP, Ausprägung der vorhandenen Allodynie sowie Grunderkrankungen wurden erfasst. Informationen über die Einnahme stabiler Medikation zur Behandlung von PSNP und Begleiterkrankungen wurden gesammelt.

Ergebnisse: Daten von 363 Patienten, davon 64\% Frauen, wurden untersucht. Das Durchschnittsalter lag bei 52 Jahren. $82 \%$ der Patienten waren zwischen 18 und 65 Jahre alt. Im Durchschnitt litten die Patienten seit 14 Monaten an PSNP. Die Schmerzintensität bei Studienstart betrug 6,6 von 11 [11-Punkt Skala, $0=$ kein Schmerz, $10=$ schlimmster vorstellbarer Schmerz]. Die Stärke der Allodynie betrug 5,8.

Die häufigsten zu PSNP führenden OPs waren KarpaltunnelsyndromOPs, OPs nach Frakturen oder Gelenkversteifungen, Wirbelsäulen-, sowie Hüft-, Leistenbruch-, Knieersatz-, und Thorax-OPs. Die Hälfte der Patienten benötigte regelmäßig stabile Schmerzmedikation: hauptsächlich Opioide, nichtsteroidale Antiphlogistika, Antiepileptika und Antidepressiva. Die häufigsten Begleiterkrankungen waren Gefäß-, Herz-Kreislaufund gastrointestinale Erkrankungen. Aufgrund dieser Begleiterkrankungen wurden Lipidsenker, Antihypertensiva und Antacida eingenommen. Schlussfolgerung: In dieser Studie gehen verschiedene Arten von Operationen der Chronifizierung postoperativer Schmerzen voraus. Patienten leiden trotz regelmäßiger Schmerzmedikamenteneinnahme unter persistierenden mittelstarken bis schweren Schmerzen. Deren Intensität und Dauer veranschaulichen den beeinträchtigenden Charakter von PSNP. Die Ergebnisse zeigen, dass ein hoher Bedarf an wirksamen und sicheren Behandlungsoptionen für PSNP besteht.

Die Autoren sind Mitarbeiter von Grünenthal. Die Studie wurde von Grünenthal finanziert.

\section{Literatur}

1. Kehlet et al (2006) Persistent postsurgical pain: risk factors and prevention. Lancet 367:1618-1625

2. Kehlet et al (2006) Prevalence of and factors associated with persistent pain following breast cancer surgery. JAMA 302:1985-1992

3. Brandborg et al (2007) Risk factors for chronic pain after hysterectomy: a nationwide questionnaire and database study. Anesthesiol 106:1003-1012

4. ClinicalTrials.gov: NCT01752322 Efficacy and Tolerability of Lidocaine Plaster for Treatment of Long-term Local Nerve Pain.

\section{P08.07 - Contralaterally different sensory profiles in patients with distal symmetrical painful polyneuropathy by quantitative sensory testing (QST)}

\section{G. Landmann, L. Stockinger, M. Béchir}

Schweizer Paraplegiker-Zentrum, Zentrum für Schmerzmedizin, Nottwil, Switzerland

Background and aims: Painful polyneuropathy of different aetiologies often presents with distal symmetrical clinical signs and symptoms. Because sensory profiling becomes a major issue in treatment of neuropathic pain quantitative sensory testing (QST) was performed bilaterally on feed to assess sensory profiles.

Methods: Thirty seven consecutive patients (19 females) with a mean age of 61 years were carefully assessed. The diagnosis of painful distal symmetrical polyneuropathy was established by a neurologist based on signs and symptoms. Half of the patients had a pain duration more than 5 years. QST was performed according to the standardized protocol of the German Research Network on neuropathic pain (DFNS) on both feet including cold detection threshold (CDT), warm detection threshold (WDT), thermal sensory limen (TSL), paradoxical heat sensation (PHS), cold pain threshold (CPT), heat pain threshold (HPT), mechanical detection threshold (MDT), mechanical pain threshold (MPT), mechanical pain sensitiv- ity (MPS), dynamic mechanical allodynia (DMA), wind up ratio (WUR), vibration detection threshold (VDT) and pressure pain threshold (PPT). Sensory function was assessed using loss and gain-score (LOGA). Ratings are L: loss, G: gain, 1: thermal symptoms, 2: mechanical symptoms, 3: ther$\mathrm{mal}$ and mechanical symptoms. Nociceptor function was judged as irritable nociceptor phenotype (preserved small fibre function, but gain in nociceptive parameters) or non-irritable nociceptor phenotype for each side. Sensory nerve conduction studies (sNCS) of sural nerves were performed. Side to side comparisons were made for sNCS and QST-parameters.

Results: sNCS was normal in 6 cases, but QST in all of these patients was abnormal. 1/3 of patients had abnormal sNCS unilateral only. QST was nor$\mathrm{mal}$ in 1 patient and unilateral abnormal in 2 patients. The most frequent abnormal QST-parameters were (unilateral versus bilateral): CDT $(13 ; 6)$, PHS (12;11), VDT (8;17), TSL (7;15), MDT (7;14), WDT (6;8), MPS $(6 ; 4)$ and MPT (4;6). Most frequent LOGA-scores were L2G0 (thermal detection loss only) found unilateral in 8 patients (bilateral in 7) and L3G0 (thermal and mechanical detection loss) found unilateral in 6 patients (bilateral 9). $1 / 3$ of patients had different LOGA-scores on both sides. Irritable nociceptor phenotype was found in 5 patients unilateral but in 4 patients bilaterally. Conclusion: Our finding suggest that in patients presenting with clinical distal symmetrical painful polyneuropathy QST shows highly heterogeneously sensory profiles not only compared inter-but also intra-individually. This might be of interest for treatment response because different sensory profiles may respond differently to anti-neuropathic treatment. Studies with regard to treatment response in patients with side to side differences of sensory profiles should be performed.

\section{P08.08 - 1187 Patienten mit Läsionen des Nervensystems mit und ohne paradoxe Hitzeempfindungen im somatosensorisches Profil}

J. Vollert ${ }^{1}$, N. Attal ${ }^{2}$, R. Baron ${ }^{3}$, D. Bouhassira ${ }^{2}$, N. Finnerup ${ }^{4}$, R. Freynhagen ${ }^{5}$, M. Haanpä̈̈6, P. Hansson ${ }^{7}$, T. Jensen ${ }^{8}$, C. Maier ${ }^{9}$, A. Rice ${ }^{10}$, M. Segerdahl ${ }^{11}$, J. Serra ${ }^{12}$, S. Sindrup ${ }^{13}$, T. Tölle ${ }^{14}$, R. Treede ${ }^{15}$

${ }^{1}$ Berufsgenossenschaftliches Universitätsklinikum Bergmannsheil $\mathrm{GmbH}$, Abt. für Schmerzmedizin, Bochum, Deutschland, ${ }^{2}$ I'hôpital ambroise paré, Centre d'Evaluation et de Traitement de la Douleur, Boulogne-Billancourt, Frankreich, ${ }^{3}$ Klinik für Neurologie, Universitätsklinikum Schleswig-Holstein, Campus Kiel, Sektion Neurologische Schmerzforschung und -therapie, Kiel, Deutschland, ${ }^{4}$ Aarhus University, Danish Pain Research Center, Aarhus C, Dänemark, ${ }^{5}$ Benedictus Krankenhaus Tutzing, Zentrum für Anästhesiologie, Intensivmedizin, Schmerztherapie \& Palliativmedizin, Tutzing, Deutschland, ${ }^{6} \mathrm{Helsinki}$ University, Departments of Helsinki University Central Hospital, Helsinki, Finnland, ${ }^{7}$ Karolinska Institut, Solna, Schweden, ${ }^{8}$ Danish Pain Research Center, Aarhus University Hospital, Dänemark, Dept. of Neurology, Aarhus, Dänemark, ${ }^{9}$ Berufsgenossenschaftliches Universitätsklinikum Bergmannsheil GmbH, Bochum, Abteilung für Schmerzmedizin, Bochum, Deutschland, ${ }^{10} \mathrm{Imperial}$ College, Chelsea and Westminster Hospital, London, United Kingdom, ${ }^{11} \mathrm{H}$. Lundbeck A/S, Valby, Dänemark, ${ }^{12}$ Neuroscience Technologies Ltd., Barcelona, Spanien, ${ }^{13}$ University of Southern Denmark, Odense M, Dänemark, ${ }^{14}$ Zentrum für interdisziplinäre Schmerztherapie, Neurologische Klinik - Klinikum rechts der Isar, TU München, München, Deutschland, ${ }^{15}$ Universität Heidelberg, Lehrstuhl für Neuropyhsiologie, Mannheim, Deutschland

Ziel der Untersuchung: Paradoxe Hitzeempfindungen (PHS), bei denen ein Patient Wärme empfindet, während ein Hautareal gekühlt wird, treten äußerst selten bei gesunden Probanden auf, aber häufig bei Patienten, die an einer Läsion oder Erkrankung des Nervensystems leiden (Maier et al., 2010). Als Mechanismus vermutet man den Verlust von A $\delta$-FaserFunktion, was zu einer Disinhibition von C-Faser-Neuronen führt (Craig and Bushnell, 1995), andererseits wurde auch ein verstärkter Verlust von C-Fasern bei Patienten mit PHS gezeigt (Hansen et al., 1996). Ziel der Untersuchung war, Parameter der Quantitativ-Sensorischen Testung (QST, Rolke et al., 2006) und Domänen des Neuropathic Pain Symptom Inventory Fragebogens (NPSI, Bouhassira et al., 2004) zu identifizieren, die mit dem Vorhandensein von PHS interagieren. Folgende vier Hypothesen sollten hierbei überprüft werden: die Frequenz von PHS ist erhöht bei 
Patienten mit (a) verringerter A $\delta$-Faser-Funktion, was sich in erhöhter Kältedetektionsschwelle (CDT) oder thermaler Detektionsschwelle (TSL) zeigt, (b) verringerter C-Faser-Funktion, was sich in erhöhter Wärmedetektionsschwelle (CDT) zeigt, (c) verringerter Kälteschmerzschwelle (CPT) oder Hitzeschmerzschwelle (HPT), (d) Schmerz, der sich durch Brenngefühl und nicht durch das Gefühl elektrischer Schocks auszeichnet.

Methoden: QST von 1187 Patienten mit schmerzhaften oder schmerzfreien Läsionen oder Erkrankungen des Nervensystems aus 15 europäischen Zentren wurden in die Untersuchung eingeschlossen. Der NPSI lag von 404 dieser Patienten vor. Die elf normalverteilten QST Parameter und 10 NPSI Domänen wurden in ungepaarten t-tests auf Unterschiede zwischen Patienten mit und ohne PHS getestet.

Ergebnisse: Patienten mit und ohne Schmerzen unterschieden sich nicht in der Frequenz auftretender PHS. Bei Patienten mit zentralem Schmerz und unilateralen peripheren Schmerzen zeigte sich bei Patienten mit PHS eine signifikant erhöhte CDT, WDT und TSL $(p<0,05)$ im Vergleich zu Patienten ohne PHS. Dieses Muster war ähnlich für schmerzfreie Neuropathien, allerdings nur für WDT signifikant, was sich durch deutlich kleinere Fallzahlen erklären lässt. Es konnten keine Unterschiede für CPT, HPT oder Brennschmerz in den Gruppen gefunden werden, die Hypothesen (c) und (d) daher verworfen. Unerwarteter Weise ließen sich keinerlei Unterschiede im QST-Profil von Patienten mit und ohne PHS bei Vorliegen einer Polyneuropathie zeigen.

Diskussion: Unsere Ergebnisse zeigen, dass PHS deutlich mit dem Verlust kleiner Fasern einhergehen, dies ist jedoch nicht spezifisch für dünn-myelinisierte $\mathrm{A} \delta$-Fasern (CDT), sondern schließt unmyelinisierte C-Fasern (WDT) ebenso ein. PHS können daher als Marker einer Kleinfaser-Neuropathie fungieren, nicht jedoch um schmerzhafte von schmerzfreien Neuropathien unterscheiden oder Brennschmerz identifizieren zu können.

\section{P08.09 - Insuffiziente Regeneration der mechanischen Nervenfunktion nach peripheren Nervenverletzungen}

\section{J. Huber', C. Maier' ${ }^{2}$, T. Mainka ${ }^{3}$, L. Mannil', J. Vollert ${ }^{5}$, H. Homann}

'Ruhr-Universität Bochum, Bochum, Deutschland,

${ }^{2}$ Berufsgenossenschaftliches Universitätsklinikum Bergmannsheil GmbH, Bochum, Abteilung für Schmerzmedizin, Bochum, Deutschland, ${ }^{3}$ University Medical Center Hambur Eppendorf, Department of Neurology, Hamburg, Deutschland, ${ }^{4}$ BG Unfallklinik Duisburg, Hand- \& Plastische Chirurgie, Brandverletzungen, Duisburg, Deutschland, ${ }^{5}$ Berufsgenossenschaftliches Universitätsklinikum Bergmannsheil GmbH, Abt. für Schmerzmedizin, Bochum, Deutschland

Thema und Zielsetzung: Periphere Nervenverletzungen an der Hand führen $\mathrm{zu}$ einem gravierenden Verlust der mechanischen und sensorischen Funktion des betroffenen Versorgungsgebietes. In dieser Studie untersuchten wir die Regeneration sowohl der dicken, myelinisierten Aß-Fasern, sowie die der dünnen, beziehungsweise unmyelinisierten $\mathrm{A} \delta$ - und $\mathrm{C}$-Fasern. Methodik: 18 periphere Nervenverletzungen mit Nervendefektstrecke, die mit einem Kollagen-Conduit behandelt wurden, sowie 16 End-zu-EndNervennähte und 30 gesunde Kontrollpersonen wurden untersucht mittels quantitativer sensorischer Testung, dem DASH- (disabilities of the arm, shoulder and hand) Fragebogen, dem painDetect-Fragebogen, sowie der Messung des Bewegungsumfangs der Gelenke der oberen Extremität.

Ergebnisse und Schlussfolgerung: Die somatosensorischen Profile nach Anwendung beider Verfahren zeigten eine größtenteils regenerierte Funktion der $\mathrm{C}$ - und $\mathrm{A} \delta$-Fasern, aber eine schwere Einschränkung der Funktion der Aß-Fasern und demnach eine deutlich erhöhte mechanische Detektionsschwelle. Trotz mangelhafter Nervenregeneration traten Schmerzen insgesamt selten auf. Wenige Patienten beider Gruppen empfanden Druckschmerz von geringer Intensität, sowie minimal Allodynie Patienten, deren Nervenverletzung mit Einsatz eines Conduits behandelt wurden zeigten sich funktionell deutlich eingeschränkter, vor allem in der Ausübung ihres Berufes, was mit dem Verlust der mechanischen Nervenfunktion korreliert.
Sensorisches Training könnte ein vielversprechender Ansatz sein um die Regeneration von Nervenfasern nach peripheren Nervenverletzungen weiter zu verbessern.

\section{P08.10 - Calciumtoxizität in der Genese der Paclitaxel-induzierten Neuropathie}

\section{Bernard, M. Leo, L. Schmitt, T. Hagenacker}

Universitätsklinikum Essen, Klinik für Neurologie, Experimentelle Schmerzforschung, Essen, Deutschland

Die Verwendung von Taxanen findet regelhaft Anwendung vor allem in der Behandlung gynäkologischer Tumoren. Eine der wichtigsten langfristigen Therapiefolgen sind schmerzhafte Polyneuropathien, die durch neurotoxische Nebenwirkungen entstehen. Die molekularen Mechanismen, die der Neurotoxizität zu Grunde liegen, sind weitgehend unverstanden, ihre Kenntnisse für die Entwicklung neuroprotektiver und innovativer analgetischer Therapieansätze jedoch essentiell. Unter der Annahme, dass Taxane die intrazelluläre Calciumhomöstase stören, wurden in einem Rattenmodell Spinalganglienneuronen für $24 \mathrm{~h}$ kultiviert und anschließend mit der Patch-Clamp Technik spannungsabhängige Calciumkanalströme isoliert.

Die Applikation von Paclitaxel führt dabei zu einer dosisabhängigen Reduktion von Calciumkanalströmen. Dabei deutet die Spannungsabhängigkeit des Effektes auf eine Beteiligung unterschiedlicher Subtypen, vornehmlich von N-Typ und L-Typ Calciumkanälen, hin. Unter Verwendung hochselektiver Kanalmodulatoren sollen in einer Folgestudie diese differenziert isoliert werden, um somit im Tiermodell durch Gabe selektiver Pharmaka die neuronale Calciumhomöostase zu stabilisieren.

\section{P09 - Neuropathischer Schmerz I}

\section{P09.01 - Two loss-of-function mutations of Nav1.8 are linked to gain-of-pain in human patients}

L. Kaluza' , M. Hampl', M. Hampl', E. Bressan' ', A. Foerster' ' D. Schmidt', B. Namer' ${ }^{2}$ I. Kurth ${ }^{4}$, B. Winner ${ }^{3}$, J. Meents' ${ }^{1}$, A. Lampert ${ }^{1}$

'Uniklinik RWTH Aachen, Institut für Physiologie, Aachen, Germany, ${ }^{2}$ Institut für Physiologie \& Pathophysiologie, Universität ErlangenNürnberg, Erlangen, Germany, ${ }^{3}$ IZFK/Friedrich-Alexander Universität Erlangen-Nürnberg, Erlangen, Germany, ${ }^{4}$ Institut für Humangenetik/ Universitätsklinikum Jena, Jena, Germany

Most of the known mutations in the voltage-gated sodium channel Nav1.8 linked to inherited pain syndromes (Faber et. al. 2012; Han et. al. 2014) induce an electrophysiological gain-of-function. This leads to neuronal hyperexcitability, which explains the severe pain symptoms in patients carrying those mutations. Surprisingly, a mutation inducing an electrophysiological loss-of-function has been described before in a patient diagnosed with erythromelalgia (Kist et al. under revision).

We use patch-clamp electrophysiology and immunostaining to investigate two Nav1.8 mutations. The mutation D1639N was detected in Patient 1 and is located in the pore forming pre-loop of domain IV (Dabby et al. 2015). The mutation R923H was found in Patient 2 and resides in the intracellular linker between domain II and III.

Both Patients have been diagnosed with small fiber neuropathy (SFN), the carrier of D1639N because of her clinical symptoms and abnormal QST, the patient harboring the $\mathrm{R} 923 \mathrm{H}$ mutation due to reduced intraepidermal nerve fiber density and typical pain characteristics. Both show late onset of their clinical symptoms (Patient 1 at 32 and Patient 2 at 57 years) suggesting a slowly ongoing process within nerve fibers. Patient 1 is also suffering from gastroparesis and esophagoparesis accompanied by severe loss of bodyweight. Patient 2 features some of the typical characteristics of IEM patients such as burning and stabbing pain, relief by cooling and physical activity as a trigger factor for pain attacks. 
For both mutations we find loss-of-function characteristics: R923H induced a potent enhancement of slow inactivation, rendering about half of Nav1.8 channels inactivated at physiological resting membrane potentials. D1639N showed markedly reduced current density and we thus assume a trafficking defect. In order to restore the current density we tried different paradigms to enhance sodium channel surface expression.

For example, incubation at $27^{\circ} \mathrm{C}$ overnight was shown to increase current density for Nav1.9 (Vanoye et. al. 2013), but we found no effect on WT and even a reduction in current density of the $\mathrm{R} 923 \mathrm{H}$ mutation. Similarly, and in contrast to reports with Nav1.1 (Rusconi et al. 2007), co-expression of the $\beta 1$-subunit reduced the current density for all constructs tested. Further studies are needed to understand how a loss-of-function mutation can induce gain-of-pain. We will examine gating changes electrophysiologically and explore likely molecular trafficking defects induced by the mutations. Therefore, we will focus on the possible interplay between the Nav1.8 channel and the ER, as well as the chaperone calnexin and the ß3 subunit. Moreover, we will examine temperature-dependent effects on trafficking of the Nav1.8 channel and its mutations as well as their tentative role in pain pathomechanisms.

\section{Literatur}

Dabby R et al (2015) "Painful small fiber neuropathy with gastroparesis: A new phenotype with a novel mutation in the SCN10A gene." Journal of Clinical Neuroscience Faber CG et al (2012) "Gain-of-function Nav1.8 mutations in painful neuropathy." Proceedings of the National Academy of Sciences of the United States of America. Han et al (2014) "The G1662S NaV1.8 mutation in small fibre neuropathy: impaired inactivation underlying DRG neuron hyperexcitability." Journal of Neurology Neurosurgery \& Psychiatry

Kist et al (under revision) "SCN10A Mutation in a Patient with Erythromelalgia Enhances C-Fiber Activity Dependent Slowing."

Rusconi et al (2007) "Modulatory proteins can rescue a trafficking defective epileptogenic Nav1.1 Na+ channel mutant."The Journal of Neuroscience

Vanoye et al (2013) “Mechanism of sodium channel NaV1.9 potentiation by G-protein signaling."The Journal of general Physiology

\section{P09.02 - Untersuchung der Kleinfasermorphologie beim Fibromyalgie-Syndrom}

\section{J. Frank', D. Evdokimov' ', D. Kampik², C. Sommer' , N. Üçeyler ${ }^{1}$}

'Universitätsklinikum Würzburg, Neurologische Klinik, Würzburg, Deutschland, ${ }^{2}$ Universitätsklinikum Würzburg, Augenklinik, Würzburg, Deutschland

Fragestellung: Das Fibromyalgie-Syndrom (FMS) ist ein chronisches Schmerzsyndrom mit muskuloskelettalen Schmerzen in mehreren Körperregionen und zusätzlichen Symptomen wie Fatigue und Schlafstörungen. Es mehren sich Hinweis auf eine Kleinfaserpathologie (Ad und C) bei FMS-Subgruppen, wobei die zugrunde liegenden Mechanismen unklar sind. Wir untersuchen mittels cornealer confocaler Mikroskopie (CCM), ob bei FMS-Patienten analog zur Reduktion der kutanen intraepidermalen Nervenfaserdichte (IENFD) auch die corneale Innervation abnimmt. Methoden: Bislang wurden 40 FMS Patienten (40 Frauen; Median 54 Jahre, 24-75) und 34 gesunde Probanden ( 31 Frauen, 3 Männer; Median 49 Jahre, 27-65) rekrutiert. Die Teilnehmer wurden klinisch-neurologisch und mittels Elektroneurographie (ENG) untersucht; zudem wurden sie mit standardisierten Schmerz-, FMS- und Depressionsfragebögen befragt. Von allen Studienteilnehmern wurden zur Bestimmung der IENFD Hautstanzbiopsate vom lateralen Unter- und Oberschenkel entnommen; die Werte wurden mit unseren Labor-internen Normwerten verglichen. $\mathrm{Zu}-$ dem wurde bei allen Studienteilnehmern mittels CCM der subbasale Nervenplexus der Cornea analysiert und die Nervenfaserdichte (NFD) und -länge (NFL) mittels CCMetrics Software (M. A. Dabbah, Imaging Science, Manchester) quantifiziert.

Ergebnisse: Die neurologische Untersuchung war bei den Patienten unauffällig; die ENG ergab keine Hinweise auf eine Polyneuropathie. In den Fragebögen hatten FMS Patienten deutlich erhöhte Scores für Schmerz und depressive Symptome $(p<0,001)$. 15/40 (38\%) der FMS Patienten (Median 4,9 Fasern/mm; $0-5,7$ ) hatten im Vergleich zu Kontrollen (Median 7 Fasern/mm; 1-15) eine reduzierte IENFD am Unterschenkel $(p<0,01)$.
Am Oberschenkel fanden sich bei den Patienten im Median 9 Fasern/ mm (2-20), bei den Kontrollen 10 Fasern/mm (4-22). Bei 8/40 (20\%) Patienten zeigte sich eine längenabhängige Reduktion der IENFD; 7/40 (18\%) Patienten hatten eine generalisierte IENFD Reduktion. Die corneale NFD lag bei den Patienten mit einem Median von 23 Fasern $/ \mathrm{mm}^{2}$ (11-37) deutlich niedriger als bei Kontrollen (28 Fasern $/ \mathrm{mm}^{2} ; 15-42$ ); auch war die NFL mit $13 \mathrm{~mm} / \mathrm{mm}^{2}(8-20)$ im Vergleich zu Kontrollen vermindert $\left(16 \mathrm{~mm} / \mathrm{mm}^{2}\right.$; je $\left.p<0,01\right)$. Weder distale, noch proximale IENFD korrelierten mit der NFD oder NFL. Schlussfolgerung: Der Befund einer reduzierten distalen IENFD bei ca. der Hälfte der FMS Patienten bestätigt sich auch in der aktuellen Studie. Die fehlende Korrelation zwischen kutaner und cornealer Innervation ist ein weiterer Hinweis auf unterschiedliche Pathomechanismen, die beim FMS zur Faserde- und -regeneration bzw. zu Schmerz beitragen.

\section{P09.03 - Somatosensory profiles differ between patients with non-specific neck arm pain with and without heightened nerve mechanosensitivity}

\section{K. Boettger ${ }^{1}$, B. Tampin ${ }^{2,3}$, L. Stockinger 4 , G. Landmann ${ }^{4}$, A. Schmid ${ }^{5}$}

${ }^{1} S c h w e i z e r$ Paraplegiker Zentrum, Zentrum fuer Schmerzmedizin, Nottwil, Switzerland, ${ }^{2}$ Sir Charles Gairdner Hospital, Department of Physiotherapy, Perth, Australia, ${ }^{3}$ Curtin University, School of Physiotherapy and Exercise Science, Perth, Australia, ${ }^{4}$ Schweizer Paraplegiker-Zentrum, Zentrum für Schmerzmedizin, Nottwil, Switzerland, ${ }^{5}$ Oxford University, Nuffield Department of Clinical Neurosciences, Oxford, United Kingdom

Background: While clinical neurological examination and electrodiagnostic studies are usually normal in patients with non-specific neck arm pain (NSNAP), a subgroup of patients with NSNAP present with clinical signs of heightened nerve mechanosensitivity. The latter is characterized clinically by pain in response to limb movements that cause nerve elongation (neurodynamic tests). It is assumed that this increased neural mechanosensitivity reflects a gain of function, presumably mediated by small nociceptive fibres. However, this assumption has not yet been verified.

Purpose: The aim of this study was to establish the somatosensory profiles of patients with unilateral NSNAP with and without heightened nerve mechanosensitivity and to compare these between groups and with healthy controls (HCs).

Methods: Twenty-eight patients with unilateral NSNAP participated in the study. All patients had no abnormality detected on electrodiagnostic testing. Patients were subdivided with standardised upper limb neurodynamic tests into those with heightened nerve mechanosensitivity (ULNT ${ }^{\text {pos }}$ ) and those with normal nerve mechanosensitivity (ULNT ${ }^{\text {heg }}$ ). Quantitative sensory testing was performed in accordance with the protocol of the German Network on Neuropathic Pain (DFNS) including measurements of thermal and mechanical detection and pain thresholds, mechanical pain sensitivity and wind up ratios. All parameters were evaluated over the patients' maximal pain area. QST data were compared to reference data obtained in $31 \mathrm{HC}$ subjects. QST data were log transformed and expressed as z-scores. Ethical approval was obtained by Swissethics (EKNZ 2014-243). Results: Seventeen patients were classified as ULNT ${ }^{\text {pos }}$ and 11 as ULNT ${ }^{\text {heg. }}$. Both groups were characterized by a loss of function in thermal detection thresholds $(p<0.003)$ and an increased windup ratio $(p<0.001)$ compared to HCs. Furthermore, patients with ULNT ${ }^{\text {pos }}$ demonstrated reduced mechanical $(p=0.001)$ and vibration detection sense $(p=0.002)$ compared to HCs. Patients with ULNT ${ }^{\text {os }}$ had significantly lower pain thresholds (cold, heat, pressure) compared to HCs $(p<0.045)$. The only parameter that differentiated patients with ULNT ${ }^{\text {os }}$ from patients with ULNT $^{\text {heg }}$ was the heat pain threshold with ULNT ${ }^{\text {pos }}$ patients being more sensitive to heat $(p=0.017)$.

Discussion: In comparison to HCs, patients with NSNAP and heightened nerve mechanosensitivity demonstrated more sensory alterations in their maximal pain area than patients with negative neurodynamic tests. The lowered heat pain threshold in patients with ULNT ${ }^{\text {pos }}$ compared to patients with ULNT ${ }^{\text {heg }}$ is indicative of a dysfunction of small unmyelinated fibres. 
Conclusion: The findings of this study suggest that heightened nerve mechanosensitivity may reflect an increased gain of function of the small fibre population. The findings contribute to an enhanced understanding of possible underlying mechanisms of heightened nerve mechanosensitivity.

\section{P09.04 - Schmerz-evozierte Potentiale bei unilateralen peripheren Nervenschäden der oberen Extremität}

M. Fischer' ', O. Höffken², Ö. Özgül' ${ }^{2}$ J. Vollert ${ }^{1}$, E. Enax-Krumova ${ }^{2}$, C. Maier ${ }^{1}$ ${ }^{1}$ Berufsgenossenschaftliches Universitätsklinikum Bergmannsheil $\mathrm{gGmbH}$, Abteilung für Schmerzmedizin, Bochum, Deutschland, ${ }^{2}$ Berufsgenossenschaftliches Universitätsklinikum Bergmannsheil gGmbH, Neurologische Klinik und Poliklinik, Bochum, Deutschland

Hintergrund und Zielsetzung: Bei einer Subgruppe von Patienten mit peripheren Nervenschäden (PNI) besteht im Vordergrund eine Schädigung der kleinkalibrigen $\mathrm{C}$ - und $\mathrm{A} \delta$-Nervenfasern, die mittels Elektroneurographie (ENG) nicht nachweisbar ist. Wir haben untersucht, ob Schmerzevozierte Potentiale (PREP) einen zusätzlichen diagnostischen Nutzen im Vergleich zu QST aufweisen.

Methode: 23 Patienten mit PNI der oberen Extremität und 38 Gesunde erhielten im Versorgungsgebiet des betroffenen Nervens und kontralateral PREP (modifiziert nach Obermann et al. 2008) und eine Quantitativ Sensorische Testung (QST) gemäß DFNS-Protokoll (Rolke et al. 2006) (Patienten zusätzlich ENG). Die Wahrnehmungs- (WS) und Schmerzschwellen (SS), N1- und P1-Latenzen und N1P1-Amplituden der PREP verglichen wir mit sensorischen Detektionsschwellen der QST. Patienten wurden beidseits mit der zweifachen SS der gesunden Seite stimuliert. Statistik: T-Test, Pearson-Korrelation.

Ergebnis: Während sich die P1-Latenzen und N1P1-Amplituden weder im Gruppen- noch im Seitenvergleich unterschieden, unterschied sich die N1-Latenz signifikant zwischen Patientengruppe (PG) und Kontrollgruppe (CG) (PG: 167,0 $\pm 40,7$ ms vs. 145,9 $\pm 17,8 \mathrm{~ms}$ ) ohne Unterschiede im Seitenvergleich. 16 der 23 Patienten $(69,6 \%)$ hatten im QST erhöhte Detektionsschwellen ( $n=13$ eine thermische Hypästhesie), im ENG lagen nur bei 8 von 21 Patienten $(38,1 \%)$ pathologische Befunde vor. Alle 8 Patienten mit erhöhter N1-Latenz (>175 ms) zeigten auch pathologische Werte in der QST und ENG.

Die WS korrelierte bei allen signifikant mit der Kälte- $(\mathrm{CDT})(\mathrm{r}=0,57$; $p<0,001)$ und Wärmewahrnehmungsschwelle (WDT) $(\mathrm{r}=0,45 ; p<0,001)$ sowie mechanischen Detektionsschwelle (MDT) $(\mathrm{r}=0,49 ; p<0,001)$, die N1-Latenz mit CDT $(\mathrm{r}=0,40 ; p<0,005)$, WDT $(\mathrm{r}=0,44 ; p<0,001)$ und $\operatorname{MDT}(\mathrm{r}=0,34 ; p<0,01)$.

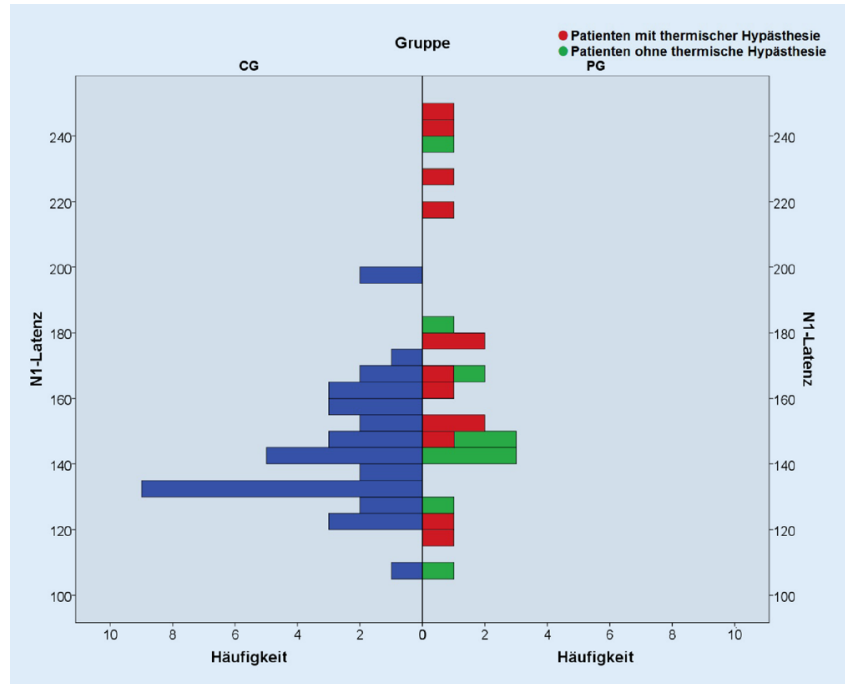

Abb. $1 \Delta$ Histogramm über die Verteilung der N1-Latenz in der CG und PG. CG Kontrollgruppe, $P G$ Patientengruppe
Schlussfolgerung: Trotz signifikanter Korrelation mit thermischen Wahrnehmungsschwellen und verlängerter N1-Latenz bei Patienten bieten PREP keinen diagnostischen Zusatznutzen bei PNI der oberen Extremität. Auch durch einen Seitenvergleich wird die Sensitivität nicht erhöht. Im Gegensatz zu systemischen Polyneuropathien (Uceyler et al. 2013, Obermann et al. 2008) scheinen bei Patienten mit PNI häufig ausreichend viele myelinisierte $\mathrm{A} \delta$-Fasern vorhanden zu sein, durch deren elektrische Reizung normale Latenzen und Amplituden erzeugt werden.

\section{Literatur}

1. Obermann et al (2007) Correlation of epidermal nerve fiber density with painrelated evoked potentials in HIV neuropathy. Pain 138(1):79-86

2. Rolke et al (2006) Quantitative sensory testing in the German Research Network on Neuropathic Pain (DFNS): standardized protocol and reference values. Pain 123(3):231-243

3. Uceyler et al (2013) Small fibre pathology in patients with fibromyalgia syndrome. Brain 136(6):1857-1867

\section{P09.05 - Einfluss von Oxaliplatin auf spannungsabhängige Calciumkanäle in DRG-Neuronen der Ratte}

\section{Schmitt, M. Leo, T. Hagenacker}

Universitätsklinikum Essen, Klinik für Neurologie, Experimentelle Schmerzforschung, Essen, Deutschland

Hintergrund: Platin-haltige Zytostatika spielen eine wichtige Rolle in der Behandlung verschiedener Krebsarten. Neben ihrer hohen Wirksamkeit in der Chemotherapie kommt es jedoch häufig zu schweren Nebenwirkungen. Eine dieser Nebenwirkungen ist eine schmerzhafte periphere Polyneuroapthie, welche die Lebensqualität der betroffenen Patienten stark einschränkt. Die genauen Mechanismen dieser Chemotherapie-induzierten Polyneuropathie sind bis heute nicht vollständig aufgeklärt, jedoch wird in der Literatur neben der Akkumulation sogenannter Platin/DNAAddukte auch eine Störung der Calcium-Homöostase der Spinalganglionneuronen (DRG-Neurone) diskutiert.

Das Ziel dieser Studie war es, den Einfluss von Oxaliplatin auf spannungsabhängige Calciumkanäle in DRG-Neuronen zu untersuchen. Hierdurch sollten Erkenntnisse zu grundlegende Mechanismen Oxaliplatin-induzierter neuropathischer Schmerzen gewonnen werden.

Methoden: Im Rahmen dieser Studie wurde der Einfluss von Oxaliplatin auf die verschiedenen Subtypen von spannungsabhängigen Calciumkanälen (VGCC) mit Hilfe von Patch-Clamp Messungen und immunozytochemischen Färbungen untersucht. Alle Experimente wurden an kultivierten DRG-Neuronen der Ratte (Wistar) durchgeführt.

Ergebnisse: Durch elektrophysiologische Messungen konnte hierbei gezeigt werden, dass die Applikation von Oxaliplatin die Ströme von VGCC Subtypen reduziert. Im Gegensatz dazu, führte die langzeitige Exposition kultivierter DRG-Neuronen mit Oxaliplatin zu einer signifikanten Erhöhung der Stromdichte von VGCC des L- und T-Typs. Subtypen des N- und P-/Q-Typs wurden hingegen nicht beeinflusst.

Die Zunahme der Stromdichte der VGCC des L- und T-Typ konnte durch immunozytochemische Färbungen mit der Erhöhung des Proteinlevel dieser Kanäle in Zusammenhang gebracht werden.

In in vitro-Apoptose-Experimenten konnte gezeigt werden, dass die Oxaliplatin-vermittelte Zunahme apoptotischen DRG-Neuronen durch die kombinierte Blockade der L-Typ und T-Typ VGCC, durch Nimodipin und Pimozid, verhindert werden konnte.

Diskussion: Durch die in dieser Arbeit durchgeführten in vitro-Experimente konnte der Einfluss von Oxaliplatin auf VGCC von DRG-Neuronen nachgewiesen werden. Die hierbei erzielten Ergebnisse deuten darauf hin, dass es sich bei der durch Oxaliplatin-vermittelten Neurotoxizität um eine Calcium-basierte Toxizität handelt. Hierdurch könnte es zu Funktionsstörungen der sensorischen Neurone kommen. Dies könnte wiederum die Genese neuropathischer Schmerzen nach Oxaliplatin-Chemotherapie begünstigen.

Dieses Projekt wird von der Deutschen Forschungsgemeinschaft, sowie der Deutschen Schmerzgesellschaft (Max von Frey Preis 2013) gefördert. 
P09.06 - Neurotoxische und neuroprotektive Mechanismen Cisplatin-induzierter schmerzhafter Polyneuropathien

\section{Leo' ', L. Schmitt ${ }^{1}$, M. Erkel' ${ }^{2}$, J. Thomale'2, T. Hagenacker ${ }^{\text {? }}$}

'Universitätsklinikum Essen, Klinik für Neurologie, Experimentelle Schmerzforschung, Essen, Deutschland, ${ }^{2}$ Universitätsklinikum Essen, Institut für Zellbiologie, DNA-Reparatur, Essen, Deutschland

Hintergrund: Platin-haltige Zytostatika spielen eine wichtige Rolle in der Behandlung verschiedener Krebsarten. Neben ihrer hohen Wirksamkeit in der Chemotherapie kommt es jedoch häufig zu schweren Nebenwirkungen. Eine dieser Nebenwirkungen ist eine schmerzhafte periphere Polyneuropathie, welche die Lebensqualität der betroffenen Patienten stark einschränkt. Die genauen Mechanismen dieser Chemotherapie-induzierten Polyneuropathie sind bis heute nicht vollständig verstanden, jedoch wird in der Literatur neben der Akkumulation sogenannter Platin/DNA Addukte auch eine Störung der Calcium-Homöostase der Spinalganglionneuronen (DRG-Neuronen) diskutiert.

Das Ziel dieser Studie war es, erste Untersuchungen der Calcium-basierten neurotoxischen und neuroprotektiven Mechanismen der Cisplatin-induzierten schmerzhaften Polyneuropathie.

Methoden: Im Rahmen dieser Arbeit sollte der Einfluss von Cisplatin auf die verschiedenen Subtypen von spannungsabhängigen Calciumkanälen (VGCC) mit Hilfe von Patch-Clamp Messungen, immunozytochemischen und immunohistochemischen Färbungen, sowie Verhaltenstestungen untersucht werden.

Ergebnisse: Durch elektrophysiologische Messungen konnte hierbei gezeigt werden, dass Cisplatin die Subtypen von VGCC unterschiedlich moduliert. So wurden die Ströme der VGCC des L-, P-/Q- und T-Typs reduziert, wohingegen eine Proteinkinase $\mathrm{C}$ vermittelte Zunahme von N-Typ VGCC beobachtet werden konnte. Durch die Exposition der kultivierten DRG-Neuronen mit Cisplatin konnte eine CaMK II-assoziierte erhöhte Stromdichte von N-Typ VGCC nachgewiesen werden. Diese Erhöhung konnte mit einem erhöhten Proteinlevel dieses Subtyps in Zusammenhang gebracht werden. Auch nach in vivo Cisplatin-Applikation zeigte sich eine signifikante Protenlevel-Erhöhung der N-Typ VGCC. In Apoptose-Experimenten zeigte sich, dass die durch Cisplatin erhöhte Apoptoserate durch die Blockade der N-Typ VGCC mit $1 \mu \mathrm{M}$ Ziconotid gesenkt werden konnte. In Verhaltenstestungen konnten durch die repetitive Gabe von Ziconotid (i.v.) frühe Anzeichen einer Cisplatin-induzierten Polyneuropathie verhindert werden.

Diskussion: Durch die in dieser Arbeit durchgeführten Experimente konnte der N-Typ VGCC als wichtiger Faktor für die Genese von schmerzhaften Polyneuropathien nach Cisplatin-Gabe identifiziert werden. Erstmalig konnte der Einfluss von Cisplatin auf die Subtypen von VGCC beschrieben werden. Durch die Blockade der N-Typ VGCC mit Ziconotid konnte die Induktion einer Polyneuropathie nach Cisplatin in vivo verhindert werden. Dies könnte bereits in naher Zukunft auch in der Therapie bzw. Prävention von Cisplatin-induzierten Polyneuropathien untersucht werden. Dieses Projekt wird von der Deutschen Forschungsgemeinschaft, sowie der Deutschen Schmerzgesellschaft gefördert.

\section{P09.07 - Detektion veränderter zentraler Schmerzverarbeitung bei Radikulopathiepatienten durch reduzierte Laser evozierte Potential Habituation}

P. Hüllemann' ', C. von der Brelie'2, G. Manthey', J. Düsterhöft' ${ }^{1}$ A. Helmers², M. Synowitz', R. Baron ${ }^{1}$

${ }^{1}$ Christian-Albrechts-Universtät Kiel, Schmerzsektion Neurologie, Kiel, Deutschland, ${ }^{2}$ Christian-Albrechts-Universtät Kiel, Abteilung für Neurochirurgie, Kiel, Deutschland

Hintergrund: Repetitive schmerzhafte Laserreize induzieren physiologische Laser evozierte Potential (LEP) Habituation, dies zeigt sich durch eine reduzierte N2/P2 Amplitude im EEG. LEP-Habituation ist reduziert im Capsaicin-Modell für periphere und zentrale Sensibilisierung und bei Patienten mit Migräne und Fibromyalgie. In dieser Studie war das Ziel den
Zeitverlauf der LEP-Habituation bei schmerzhafter Radikulopathie zu untersuchen, einem typischen neuropathischen Schmerzsyndrom.

Methoden: Bei 27 Patienten mit schmerzhafter Radikulopathie wurden vier Blöcke à 25 Laserreize auf der Seite der ausstrahlenden Schmerzen appliziert, im Bereich des ventralen Oberschenkels (L3 Dermatom). Die Einschlusskriterien waren wie folgt: (1) mindestens ein neurologisches Radikulopathiesymptom, (2) Rückenschmerzen mit Ausstrahlung in den Fuß, (3) Nachweis einer einseitigen Kompression der L5 und/oder S1 Wurzel im MRT. Zeichen der peripheren Sensibilisierung (Hitzehyperalgesie) und Zeichen der zentralen Sensibilisierung (mechanisch dynamische Allodynie und mechanische Hyperalgesie) wurden im betroffenen Areal im L5 oder S1 Dermatom mittels Quantitativ Sensorischer Testung (QST) erfasst. Ergebnisse: Patienten mit schmerzhafter Radikulopathie wiesen eine reduzierte LEP-Habituation auf im Vergleich zu Größen- und Alters-gematchten Kontrollen. Patienten mit Zeichen einer zentralen Sensibilisierung zeigten eine noch deutlicher reduzierte LEP-Habituation innerhalb der Patientengruppe.

Schlussfolgerung: LEP-Habituation ist bei schmerzhafter Radikulopathie reduziert, als Hinweis auf eine gestörte zentrale Schmerzverarbeitung. Zentrale Sensibilisierung scheint bei der Entstehung einer pathologisch reduzierten Habituation beteiligt zu sein. Das LEP Habituationsparadigma könnte ein nützliches klinisches Tool zur Erkennung veränderter zentraler Schmerzverarbeitung bei neuropathischen Schmerzsyndromen sein. Diese Forschung wurde ermöglicht mit der freundlichen Unterstützung der Grünenthal $\mathrm{GmbH}$.

\section{P09.08 - Validierung eines neuen Thermotestgeräts bei Gesunden und Patienten mit Diabetes mellitus}

D. Pfau' , K. Doppler ${ }^{2}$, W. Greffrath ${ }^{3}$, M. Kramer', C. Maier', C. Ohler ${ }^{3}$, M. Orth ${ }^{5}$, C. Sommer ${ }^{2}$, R. Treede', S. Schuh-Hofer ${ }^{3}$

${ }^{1}$ Medizinische Fakultät Mannheim der Universität Heidelberg, Mannheim Institute for Public Health, Lehrstuhl für Neurophysiologie, Mannheim, Deutschland, ${ }^{2}$ Universitätsklinkum Würzburg, Neurologische Klinik, Würzburg, Deutschland, ${ }^{3}$ Medizinische Fakultät Mannheim der Universität Heidelberg, Mannheim, Lehrstuhl für Neurophysiologie, Zentrum für Biomedizin und Medizintechnik Mannheim, Mannheim, Deutschland, ${ }^{4}$ Berufsgenossenschaftliches Universitätsklinikum Bergmannsheil $\mathrm{GmbH}$, Bochum, Abteilung für Schmerzmedizin, Bochum, Deutschland, 5Universitätsklinikum Ulm, Klinik für Neurologie, Ulm, Deutschland

Die Untersuchung der Funktion von Nervenfasern mit Hilfe eines Thermotestgeräts ist ein zuverlässiges Testverfahren, das fester Bestandteil des Protokolls der Quantitativen Sensorischen Testung (QST) des Deutschen Forschungsverbunds Neuropathischer Schmerz (DFNS) ist. Ein Problem für die flächendeckende klinische Anwendung sind die hohen Anschaffungskosten eines solchen Geräts.

Im Rahmen dieser Studie wurde das tragbare und preisgünstigere Thermotestgerät,Q-Sense (Medoc, Israel) entwickelt und dessen Funktion durch Vergleich mit dem etablierten Thermotestgerät ,TSA (Medoc, Israel) im Rahmen einer multizentrischen Studie an 121 gesunden Probanden (62?, 59?, Alter $35 \pm 15$ Jahre) und 83 Patienten mit Diabetes mellitus (38?,45?, Alter $58 \pm 13$ Jahre) validiert. Ein wesentlicher Unterschied des Q-Sense im Vergleich zum TSA ist die Limitierung der Kühlung auf $20^{\circ} \mathrm{C}$ statt $0^{\circ} \mathrm{C}$. Es wurde das QST-Protokoll des DFNS an zwei aufeinanderfolgenden Tagen zur gleichen Uhrzeit am Fußrücken der dominanten Seite bzw. der stärker betroffenen Seite getestet. Die Reihenfolge des Q-Sense bzw. TSAEinsatzes war balanciert und einfach verblindet. Daten sind als MW \pm SEM berichtet und wurden mittels gepaartem t-test miteinander verglichen. Es zeigten sich keine signifikanten Unterschiede bei Gesunden bei der Kältedetektionsschwelle (CDT; TSA: $2,01^{\circ} \mathrm{C}$ Differenz zur Baselinetemperatur von $32{ }^{\circ} \mathrm{C}$ (lg: 0,302 $\left.\pm 0,024\right)$; Q-Sense: $2,03{ }^{\circ} \mathrm{C}$ (lg: 0,307 $\left.\pm 0,026\right)$; $p=0,922)$ und Wärmedetektionsschwelle (WDT; TSA: $4,41^{\circ} \mathrm{C}$; (lg: $0,645 \pm 0,021)$; Q-Sense: $4,75^{\circ} \mathrm{C}$ (lg: $\left.0,677 \pm 0,022\right)$; $p=0,264$ ).

Bei Testung der Patienten zeigte sich kein signifikanter Unterschied bei Testung der Wärmedetektion (TSA: $9,18^{\circ} \mathrm{C}$ (lg: 0,963 $\left.\pm 0,027\right)$; Q-Sense: $9,39^{\circ} \mathrm{C}$ (lg: 0,9672 $\left.\pm 0,026\right) ; p=0,443$ ), jedoch für die Kältedetektion (TSA: 
5,85 ${ }^{\circ} \mathrm{C}$ (lg: 0,767 $\left.\pm 0,047\right)$; Q-Sense: $5,10{ }^{\circ} \mathrm{C}$ (lg: 0,708 $\left.\left.\pm 0,040\right) ; p=0,035\right)$. Dabei war die Korrelation hoch (CDT: $r=0,77$; WDT: $r=0,89)$. Den gerätespezifischen cut-off Wert erreichten 4 Patienten für den TSA $\left(0^{\circ} \mathrm{C}\right)$ und 14 Patienten für den Q-Sense $\left(20^{\circ} \mathrm{C}\right.$; Yates-Chi2: $p=0,025$. Auf Z-Wert Ebene zeigt sich, dass mit dem TSA 21 Patienten (25,3\%) und mit dem Q-Sense 13 Patienten (15,3\%) im Vergleich zu alters- und geschlechtsgematchten gesunden Probanden einen pathologischen Wert im Sinne eines Sensibilitätsverlusts für Kälte aufweisen $\left(p=0,178\right.$; Yates-Chi $\left.{ }^{2}\right)$.

Es zeigt sich, dass die Werte des Q-Sense grundsätzlich mit denen des TSA vergleichbar waren und der Q-Sense im klinischen Einsatz für den Nachweis von Sensibilitätsverlusten für Wärme und Kälte bezogen auf das DFNS-Normkollektiv geeignet war. Eine Ausnahme bildet die Gruppe von Männern älter als 40 Jahre, deren Normbereich größer als der Cut-off Wert des Q-sense ist.

Förderung: Diese Studie wurde vom Bundesministerium für Bildung und Forschung gefördert (Förderkennzeichen: 1316062B).

\section{P09.09 - Contact heat evoked potentials: segmental acquisition from the lower extremities}

\section{J. Rinert, P. Scheuren, P. Hostettler, M. Hupp, J. Rosner, M. Hubli, C. Jutzeler, A. Curt \\ Universitätsklinik Balgrist, Zentrum für Paraplegie, Zürich, Switzerland}

Introduction: Length-dependent peripheral neuropathies are a common neurological disorder leading to sensory impairments in the lower and upper extremities. These polyneuropathies can affect the large diameter and/ or small diameter fibres. Measures of large fibre function such as nerve conduction studies, F-waves or somatosensory evoked potentials can be readily used to disclose pathology. However, the diagnosis of small-fibre neuropathies (SFN) is challenging and the common assessment methods (i.e. Quantitative Sensory Testing, Intraepidermal Nerve Fibre Density) constitute notable limitations (e.g., time-consuming, invasive). Contact heat evoked potentials present a non-invasive technique to assess thermo-nociceptive afferents in the peripheral nervous system and along the spinal-thalamo-cortical axis. So far, CHEPs are not routinely used in SFN diagnosis and their acquisition from the lower extremities is rather challenging. Lastly, there is a lack of normative data. Thus, the primary objective of the present study was to assess the feasibility of CHEPs acquisition from dermatomes of the lower extremities in healthy controls.

Methods: A total of 47 healthy control individuals between $18-80$ years were included. Nerve conduction studies were performed and in case of pathological findings participants were excluded. The study protocol consisted of unilateral assessments of pinprick, light touch, warm detection (WDT) and heat pain thresholds (HPT), followed by the recording of CHEPs. Stimulation sites were the L5 (dorsum of the foot), L2 (medial thigh) as well as the $\mathrm{S} 2$ (five fingers above the popliteal fossa) dermatomes. The order of examination and body site tested was randomized. The vertex potentials were recorded from $\mathrm{Cz}$ electrodes.

Results: Contact heat stimulation of the L2, L5 and S2 dermatome reliably elicited cortical potentials (i.e. N2P2 component). The latencies of the N2 component of the N2/P2 vertex potential showed length dependency, being the longest at the most distal stimulation (L5 dermatome: $460 \pm 46.6$ $\mathrm{ms}$ ). L2 and S2 showed latencies of $416 \pm 33.6$ and $422.4 \pm 33.5$, respectively. Using increased baseline stimulation led to a decrease in the N2 latency as well as an increase in N2/P2 amplitude. Ratings of perceived intensity were higher using the increased baseline protocol. Age correlated negatively with amplitude and positively with latency.

Conclusion: Here we show that recording CHEPS from the lower extremities is feasible. Moreover, employing the increased baseline protocol markedly improved the acquisition of CHEPs. This is likely due to a reduction in peripheral latency jitter leading to a more synchronous afferent volley within the excited fibres. The establishment of normative data will be meaningful for improving the applicability of CHEPS for the diagnosis of small-fibre neuropathies.

\section{P09.10 - Eine erhöhte Schmerzintensität ist assoziiert mit geringerer Herzfrequenzvariabilität in der frühen Phase des Komplexen Regionalen Schmerzsyndroms (CRPS)}

N. Kumowski', T. Hegelmaier ${ }^{1}$, J. Dietrich ${ }^{2}$, T. Mainka ${ }^{3}$, J. Vollert ${ }^{1}$, C. Maier ${ }^{1}$ ${ }^{1}$ Berufsgenossenschaftliches Universitätsklinikum Bergmannsheil $\mathrm{GmbH}$, Bochum, Abteilung für Schmerzmedizin, Bochum, Deutschland, ${ }^{2}$ Berufsgenossenschaftliche Universitätsklinik Bergmannsheil GmbH, Ruhr University Bochum, Medizinische Universitätsklinik I - Allgemeine Innere Medizin, Endokrinologie und Diabetologie, Gastroenterologie und Hepatologie, Bochum, Deutschland, ${ }^{3}$ University Medical Center Hamburg Eppendorf, Department of Neurology, Hamburg, Deutschland

Eine autonome Dysregulation wird für die Entstehung und Aufrechterhaltung des Komplexen Regionalen Schmerzsyndroms (CRPS) als möglicher Mechanismus diskutiert. Neben autonomen Störungen, wie verändertem Blutfluss, gestörter Thermoregulation und Sudomotorik sowie einer erhöhten adrenergen Sensitivität, gibt es Hinweise auf eine autonome Imbalance in fortgeschrittenem CRPS [1]. Das Ziel unserer Studie war es zu prüfen, ob eine autonome Dysfunktion in der frühen Phase der Erkrankung vorliegt und ob Diese mit der Schmerzintensität und anderen klinischen Parametern korreliert.

Bei 17 Patienten mit CRPS, mit einer Krankheitsdauer von unter einem Jahr (Alter: $50 \pm 12$ Jahre; Männer: 6; Erkrankungsdauer: $23 \pm 14$ Wochen; durchschnittlicher Schmerz: $4 \pm 2$ NRS $0-10)$ und 17 in Alter und Geschlecht gematchten Probanden wurde eine Herzfrequenzvariabilitätsanalyse durchgeführt.

Dabei wurde ein EKG abgeleitet (5 Min., im Liegen) und mit dem Programm ProSciCard analysiert. Die Herzfrequenz (HR), der Heart-RateVariability-Index (HRV-Index), der durchschnittliche RR-Abstand in ms (RR-Intervall) und die Standardabweichung aller RR-Intervalle (SDRR, Gesamtvariabilität) wurden als Maß für die Herzfrequenzvariabilität berechnet. Mit Hilfe eines autoregressiven Modells wurde anhand der R-R-Abstände eine Spektralanalyse durchgeführt $(\mathrm{LF}=$ low frequency, reflektiert parasympathischen und sympathischen Einfluss; HF = high frequency, reflektiert parasympathischen Einfluss; LF/HF-ratio, Maß für sympathiko-vagale Balance).

Es zeigte sich kein signifikanter Unterschied zwischen Patienten mit CRPS und Probanden (HR: Gesunde 67 \pm 13 , CRPS $69 \pm 7$; HRV-Index: Gesunde $13 \pm 4$, CRPS $11 \pm 3$; RR-Intervall: Gesund $932 \pm 186$, CRPS $876 \pm 96$; SDRR: Gesunde $50 \pm 33$, CRPS $37 \pm 15$; LF/HF-Ratio: Gesunde 1,6 $\pm 1,2$, CRPS $2 \pm 2 ; p=$ n.s.). Jedoch zeigte sich eine inverse Korrelation des mittleren Schmerzes der letzten 4 Wochen und des HRV-Index $(r=-0,624, p=0,007)$ sowie des Durchschnittsschmerzes und der Standardabweichung aller RRIntervalle $(r=-0,497, p=0,042)$. Die LF/HF-Ratio korrelierte positiv mit der Krankheitsdauer $(\mathrm{r}=0,643, p=0,013)$.

Anders als in bisherigen Studien konnten wir im Vergleich zu Probanden keine Hinweise auf eine Imbalance des autonomen Nervensystems finden. Dennoch zeigte sich, dass eine erhöhte mittlere Schmerzintensität mit einer geringeren Herzfrequenzvariabilität einhergeht. In Übereinstimmung mit Terkelsen et al. [1] weißt die positive Korrelation zwischen LF/HF-Ratio und der Krankheitsdauer auf eine zunehmende autonome Imbalance im Laufe der Erkrankung hin.

Weitere Studien sollten den Unterschied der autonomen Imbalance zwischen früher und später Phase des CRPS berücksichtigen. Außerdem sollte im Vergleich zu anderen chronischen Schmerzen zusätzlich geprüft werden, ob unser Ergebnis eine CRPS-spezifische Veränderung darstellt.

\section{Literatur}

1. Terkelsen AJ, Mølgaard H, Hansen J, Finnerup NB, Krøner K, Jensen TS (2012) Heart rate variability in complex regional pain syndrome during rest and mental and orthostatic stress. Anesthesiology 116:133-146 
P09.11 - Modulierbarkeit von Schmerz durch Small-fiberMatrixstimulation nach Konditionierung durch Capsaicin: Identifizierung einer Sham Frequenz

K. Dreher ${ }^{1}$, V. Peuckmann-Post ${ }^{1}$, R. Rolke ${ }^{2}$

'Universitätsklinikum RWTH Aachen, Palliativmedizin, Aachen, Deutschland, ${ }^{2}$ Universitätsklinikum Aachen (UKA), Direktor der Klinik für Palliativmedizin, Aachen, Deutschland

Hintergrund: Intrakutane Capsaicin-Injektionen stellen ein humanes Surrogatmodell für neuropathische Schmerzsyndrome dar und induzieren eine Sensibilisierung von WDR-Neuronen im Hinterhorn des Rückenmarks. Mit einer neuartigen Matrixstimulation lassen sich hoch- oder niederfrequente Stromreize über schmerzhaften Arealen applizieren, um die Schmerzempfindlichkeit zu modulieren. Bei niederfrequenter Matrix-Stimulation $(1-4 \mathrm{~Hz})$ kann eine Langzeitdepression im Schmerzsystem ausgelöst werden, während eine hochfrequente Stimulation um $100 \mathrm{~Hz}$ eine Langzeitpotenzierung auslöst.

Ziele: Um randomisierte kontrollierte Studien im Capsaicin-Modell mit einer Matrixelektrode durchführen zu können, ist die Identifizierung einer intermediären Sham-Frequenz als Kontrollbedingung notwendig.

Methoden: Mit und ohne Capsaicin wurden $30 \mathrm{~Hz}, 40 \mathrm{~Hz}$ und $50 \mathrm{~Hz}$ als intermediäre Frequenzen für eine Matrixstimulation gewählt. Die Empfindlichkeit gegenüber Nadelreizen wurde im konditionierten Areal am volaren Unterarm mittels der mechanischen Schmerzsensitivität (SR Funktion) gegenüber Nadelreizen quantifiziert.

Ergebnisse: Nach Konditionierung mit Capsaicin intrakutan führte eine Matrix-Stimulation mit $50 \mathrm{~Hz}$ zu einer weiteren Steigerung der Empfindlichkeit gegenüber Nadelreizen, während es unter $30 \mathrm{~Hz}$ zu einer leichten Reduktion der Schmerzsensitivität in diesem Modell kam. Die Matrixstimulation mit $40 \mathrm{~Hz}$ zeigte keine signifikante Schwellenveränderung für diesen Surrogat-Parameter.

Konklusion: Eine Matrixstimulationsfrequenz von $40 \mathrm{~Hz}$ wurde als optimale Sham-Frequenz für eine Testung mit Nadelreizen nach Konditionierung mit Capsaicin identifiziert.

\section{P10 - Psychologie und Psychotherapie des Schmerzes}

\section{P10.01 - Valenz und Arousal-Werte visueller Stimuli und ihre Rolle in der Linderung von Schmerzen: Was ist die Kraft der Bilder?}

\section{G. Külz' , M. Shaygan'², A. Böger ${ }^{3}$, B. Kröner-Herwig ${ }^{4}$}

${ }^{1}$ RKH Kassel, Klinik für Schmerzmedizin, kassel, Deutschland, ${ }^{2}$ Georg-EliasMüller-Institut für Psychologie, Klinische Psychologie u. Psychotherapie, Göttingen, Deutschland, ${ }^{3}$ Rotes Kreuz Krankenhaus Kassel Gemeinnützige $\mathrm{GmbH}$, Klinik für Schmerztherapie, Kassel, Deutschland, ${ }^{4}$ Georg-Elias-MüllerInstitut für Psychologie, Klinische Psychologie u. Psychotherapie, Göttingen, Deutschland

Die vorliegende Studie untersucht die schmerzmindernde Wirkung von Bildern mit unterschiedlichen Inhalten, welche chronischen Schmerzpatienten in vollstationärer Behandlung in einer Schmerzklinik dargeboten wurden. Untersucht wurde wie die Valenz der Bilder, Arousal sowie die Veränderung der subjektiv erlebten sozialen Unterstützung die schmerzdämpfenden Effekte der Bilder mediieren. 88 Patienten mit chronischen Schmerzen nahmen an vier aufeinanderfolgenden Tagen an der Untersuchung teil. Mit Hilfe eines digitalen Foto-Albums wurden den Patienten Fotos von geliebten Angehörigen, Landschaften oder optischen Täuschungen präsentiert.

Die Patienten stuften je unmittelbar vor und nach dem Betrachten der Bilder ihre Schmerzintensität wie auch das affektive und sensorische Schmerzerleben ein. Valenz sowie durch die Bilder verursachtes „Arousal“ wurden ebenfalls erhoben. Vor und nach Teilnahme an der Studie machten Patienten ebenfalls Angaben zu ihrer subjektiven Einschätzung ihrer sozialen Unterstützung. Die Bilder entsprachen unterschiedlichen Valenzen; Fotos Angehöriger lösten dabei die meisten positiven Gefühle aus. Alle Bilder führten nach ihrer Präsentation zu einer Verminderung der affektiven wie auch sensorischen Qualität der Schmerzen. Das Betrachten von Bildern nahestehender Angehöriger führte im Vergleich zum Betrachten der anderen Bilder zu einer signifikant ausgeprägteren Reduktion der Schmerzen. Die Valenz der Bilder mediierte den Zusammenhang zwischen Typ des Bildes und dem Ausmaß der Reduktion der Schmerzintensität. Erstmalig konnten wir so die Kraft der Bilder in der Linderung chronischer Schmerzen im stationären Umfeld demonstrieren. Das Verfahren könnte systematisch in der Behandlung chronischer Schmerzen genutzt werden, möglicherweise in bestimmten Situationen, in welchen die Schmerzen als sehr stark erlebt werden.

\section{P10.02 - Wirksamkeit kognitiver Verhaltenstherapie im Behandlungsschwerpunkt "Chronische Schmerzen" einer Hochschulambulanz}

\section{K. Schulz', T. Klan}

1Johannes Gutenberg-Universität, Psychologisches Institut, Mainz, Deutschland

Hintergrund: Die psychologische Schmerztherapie hat sich bei Patienten mit chronischen Schmerzen in einer Vielzahl von Forschungsarbeiten als wirksam erwiesen. Vor allem die psychologische Behandlung basierend auf den Prinzipien der kognitiven Verhaltenstherapie (KVT) zeigte bei verschiedenen Patientengruppen und Schmerzbildern in randomisiertkontrollierten Studien und Metaanalysen überwiegend positive Effekte. Nachweise zur Effektivität der KVT unter den Bedingungen der Routineversorgung liegen vergleichsweise selten vor. Daher wurde an einer naturalistischen Stichprobe mit heterogenen Schmerzbildern die Wirksamkeit der KVT im Behandlungsschwerpunkt für chronische Schmerzen einer deutschen Hochschulambulanz überprüft.

Methode: Die Intention-to-Treat (ITT)-Stichprobe setzte sich aus $N=31$ konsekutiven Patienten mit der Hauptdiagnose einer chronischen Schmerzstörung (DSM-IV) bei heterogenen Lokalisationen des Hauptschmerzes bzw. heterogenen Schmerzsyndromen zusammen (davon 22,6\% Fibromyalgiesyndrom, 22,6\% Ganzkörperschmerzen, 19,4\% Rückenschmerzen und 19,4\% Kopfschmerzen). Die Patienten wurden zwischen 2012 und 2015 im Schmerzschwerpunkt einer Hochschulambulanz kognitiv-verhaltenstherapeutisch behandelt. Regulär beendet wurde die Therapie von $N=23$ Patienten. Als Outcome-Maße werden routinemäßig die beiden Subskalen zum affektiven und sensorischen Schmerzerleben der Schmerzempfindungsskala (SES), die Skalen des Fragebogen zur Erfassung der Schmerzverarbeitung (FESV) sowie der Pain Disability Index (PDI) eingesetzt. Es wurden Prä-Post-Effektstärken und die Response nach dem Reliable Change Index (RCI, 95\%-Konfidenzintervall) berechnet.

Ergebnisse: Für die ITT- und Completer-Stichprobe ergaben sich signifikante Mittelwertunterschiede zwischen der Prä- und Postmessung in Bezug auf alle Outcome-Maße. Für die ITT-Stichprobe zeigten sich Effektstärken (Cohens $d$ ) zwischen $d=0,39$ und $d=0,77$, für die Completer zwischen $d=0,39$ und $d=0,99$. Die tendenziell höchsten Effektstärken lagen in Bezug auf eine Verbesserung der kognitiven Schmerzbewältigung und eine Reduktion der schmerzbedingten psychischen Beeinträchtigung vor. Den Responsestatus (ITT-Stichprobe) erreichten je nach OutcomeMaß 22,6\% bis 58,1\%, eine signifikante Verschlechterung zeigte sich bei 6,5\% (schmerzbedingte psychische Beeinträchtigung) bzw. 3,2\% (affektives Schmerzerleben).

Schlussfolgerung: Die errechneten Effektstärken liegen im mittleren bis hohen Bereich. Die Ergebnisse zeigen, dass sich die KVT bei chronischen Schmerzen auch im naturalistischen Setting einer Hochschulambulanz als wirksam erweist. Im Rahmen zukünftiger Forschung sollte untersucht werden, ob diese Effekte auch langfristig stabil bestehen. Außerdem sind bei Vorliegen einer größeren Stichprobe Subgruppenanalysen im Hinblick auf verschiedene Schmerzsyndrome geplant. 


\section{P10.03 - Kognitive Leistungsfähigkeit bei chronischen Schmerzpatienten}

\section{A. Gärtner, B. Schönbach, A. Körner, A. Preißler, M. Klotzsche, R. Sabatowski, U. Kaiser \\ UniversitätsSchmerzCentrum, Universitätsklinikum „Carl Gustav Carus", Dresden, Deutschland}

Einführung: Berichte von Schmerzpatienten sowie Ärzten und Therapeuten geben zahlreiche Hinweise auf das Vorliegen kognitiver Defizite bei chronischen Schmerzpatienten. Die Studien, die bislang unter dem Aspekt kognitiver Defizite bei chronischen Schmerzpatienten vorliegen, lassen keine einheitlichen Ergebnisse erkennen. Ziel der aktuellen Studie ist es, die kognitive Leistungsfähigkeit bei chronischen Schmerzpatienten im Rahmen eines interdisziplinären multimodalen Therapiesettings zu untersuchen.

Methode: Untersucht wurde in einem ersten Schritt das Vorliegen kognitiver Defizite bei chronischen Schmerzpatienten zu Therapiebeginn (T1) sowie in einem zweiten Schritt die Veränderung der kognitiven Leistungsfähigkeit über den Therapieverlauf (Therapiebeginn bis 10 Wochen nach Therapieende (T3)). 147 Patienten (ø 50,9 Jahre, 74,8\% weiblich, 41,5\% Realschulabschluss, 42,9\% MPSS II) der Schmerztagesklinik absolvierten in dem Zeitraum von 01/2014-01/2016 eine standardisierte computergestützte kognitive Leistungstestung (COGBAT (S. Aschenbrenner et al.)). Unter Einbezug der erhobenen kognitiven Leistungsparameter (Aufmerksamkeitsleistung, kognitive Flexibilität, Informationsverarbeitungsgeschwindigkeit, Fähigkeit ungewollte Reaktionen zu unterdrücken, Arbeitsgedächtnis, geteilte Aufmerksamkeit) wurde der Friedman-Test gerechnet (SPSS 23.0).

Ergebnisse: Für T1 zeigte sich entsprechend der Normstichprobe die Mehrheit der Patienten (in Abhängigkeit des erhobenen kognitiven Leistungsparameters zwischen $41,5 \%$ bis $51 \%$ ) als durchschnittlich (Prozentrang $(P R)=25$ bis 84$)$. Über den Therapieverlauf konnte nachgewiesen werden, dass sich, bis auf die Aufmerksamkeitsleistung, alle erhobenen $\mathrm{Pa}$ rameter signifikant positiv verändern $\left(\mathrm{Chi}^{2}=6,914\right.$ bis $\left.23,450, p<0,009\right)$. Lerneffekte können auf Grund des zeitlichen Abstandes zwischen beiden Messungen (14 Wochen) ausgeschlossen werden.

Schlussfolgerung: In der Patientenstichprobe ließen sich keine Auffälligkeiten bezüglich der Normstichprobe zu T1 feststellen. Über den Verlauf haben sich mit dem multimodalen Therapiesetting kognitive Parameter positiv verändert. Implikationen, die sich aus diesen Ergebnissen für die Therapie ergeben, müssen in weiteren Analysen geprüft werden.

\section{Literatur}

Aschenbrenner S, Kaiser S, Pfüller U, Roesch-Ely D, Weisbrod M, Kognitive Basistestung (COGBAT) ๑ SCHUHFRIED GmbH

\section{P10.04 - Moduliert psychologischer Stress Placebo- und Nocebo- Effekte auf viszeralen Schmerz und Stuhldrang?}

\section{S. Benson', T. Roderigo' , P. Enck' ${ }^{2}$, S. Elsenbruch ${ }^{1}$ \\ 'Universitätsklinikum Essen, Institut für Medizinische Psychologie und Verhaltensimmunbiologie, Essen, Deutschland, ${ }^{2}$ Universitätsklinikum Tübingen, Innere Medizin Vl, Klinik für Psychosomatik und Psychotherapie, Tübingen, Deutschland}

Hintergrund: In den vergangenen Jahren wurden Mechanismen der Placebo-Analgesie auch für viszeralen Schmerz umfassend untersucht, wohingegen Nocebo-Effekte nur selten Gegenstand von Studien waren. Obwohl Stuhldrang ein weiteres klinisch relevantes Symptom insbesondere funktioneller gastrointestinaler Erkrankungen ist, existieren bislang keine Studien zur Modulation durch Placebo- oder Nocebo-Interventionen. Daher war es ein Ziel dieser Studie, Placebo- und Nocebo-Effekte auf viszeralen Schmerz und auf Stuhldrang zu untersuchen. Ein zweites Ziel lag in der Untersuchung der Fragestellung, ob Placebo- und Nocebo-Effekte auf viszeralen Schmerz und Stuhldrang durch psychologischen Stress moduliert werden.

Methoden: Bei $N=120$ gesunden männlichen und weiblichen Probanden wurden viszerale Distensionen individuell so kalibriert, dass sie Stuhl- drang bzw. viszeralen Schmerz induzierten. In der Baselinephase wurden die Distensionen wiederholt appliziert und mittels VAS bewertet. Anschließend wurden die Probanden entweder in eine Stressbedingung (Trier Social Stress Test, $N=60$ ) oder in eine Kontrollbedingung (einfache kognitive Aufgabe, $N=60$ ) randomisiert. Danach erhielten die Probanden beider Bedingungen wiederum randomisiert eine positive (symptomlindernd, Placebogruppe), eine negative (symptomverstärkend, Nocebogruppe) oder eine wahrheitsgemäß neutrale (Kontrollgruppe) Aufklärung zu einer intravenös verabreichten „Medikation“ (de facto NaCl-Lösung). Nach dieser Erwartungsinduktion wurden abschließend identische Distensionen wie in der Baseline appliziert und mittels VAS bewertet (Testphase). Stress-Effekte wurden mittels Fragebogen (State Anxiety), Herzrate und Speichelcortisol erfasst.

Ergebnisse: In der Stressbedingung waren signifikante Anstiege von State Anxiety $(p<0,001)$, Herzrate $(p=0,035)$ und Speichelcortisol $(p<0,001)$ beobachtbar. Die Aufklärung zur Infusion führte zu signifikant unterschiedlichen Bewertungen von Stuhldrang und Schmerz in der Placebo-, Nocebo- bzw. Kontrollgruppe ( $p<0,001$, Bedingungseffekt). Interessanterweise wurde für den Stuhldrang dieser Effekt der Erwartungsinduktion durch Stress moduliert ( $p<0,05$, Interaktion). So wurde der Stuhldrang nur in der Stressbedingung durch eine positive Aufklärung reduziert $(p<0,025)$ und durch eine negative Aufklärung erhöht $(p=0,026)$. Viszeraler Schmerz war nach positiver Information sowohl in der Stress$(p<0,038)$ als auch in der Kontrollbedingung $(P=0,043)$ reduziert. Eine Nocebo-Hyperalgesie war hingegen nur in der Stressbedingung beobacht$\operatorname{bar}(p=0,009)$.

Schlussfolgerungen: Diese „proof-of-concept“-Studie zeigt, dass Stuhldrang und viszeraler Schmerz durch verbal induzierte Erwartungen modifizierbar sind, wobei sowohl Placebo- als auch Nocebo-Effekte auf den Stuhldrang durch Stress verstärkt wurden. Zusammenfassend unterstützen die Befunde die Bedeutung psychologischer Prozesse bei der Intensivierung von Stuhldrang und Schmerz als zentrale Symptome zahlreicher gastrointestinaler Erkrankungen.

Förderung: DFG FOR 1328, EL 236/8-2.

\section{P10.05 - Einfluss von Bindungsverhalten auf chronischen und somatoformen Schmerz}

\section{A. Pfeifer, M. Schiltenwolf}

Zentrum für Orthopädie, Unfallchirurgie und Paraplegiologie Universitätsklinikum Heidelberg, Konservative Orthopädie und Schmerztherapie, Heidelberg, Deutschland

Hintergrund: In der Bindungsforschung wird der Bindungsstil als psychosozialer Vulnerabilitätsfaktor für eine Chronifizierung von akuten Schmerzen angesehen. Es wird zwischen folgenden Bindungsstilen unterschieden: sicher, anklammernd, ängstlich und ängstlich-vermeidend. Das Persönlichkeitsmerkmal des individuellen Bindungsverhaltens beeinflusst nicht nur das Schmerzerleben und -verhalten von Patienten, es wirkt sich auch darauf aus, wie stark sie insgesamt von einer Schmerztherapie profitieren. Unter anderem konnte gezeigt werden, dass sicher gebundene Patienten besser in der Lage sind, Therapieerfolg über einen längeren Zeitraum aufrecht zu erhalten im Vergleich zu unsicher gebundenen Patienten. Diese Ergebnisse sind besonders relevant, da Patienten mit chronischen Schmerzen in der Gruppe der unsicher Gebundenen überrepräsentiert sind. Damit erweist sich der Bindungsstil als lohnendes Forschungsfeld im Zusammenhang mit der Behandlung von Schmerzzuständen.

Methoden: Dafür wurden die Datenbanken Pubmed, Medline und Psycinfo auf die Begriffe „Attachment" und „Pain“ durchsucht. Die Suche ergab 50 Artikel in Psycinfo, 58 in Pubmed und 54 in Medline. Es wurden nur Studien verwendet, bei welchen Patienten entweder unter chronischen Schmerzen litten oder die Diagnose somatoforme Schmerzen erhalten hatten und die zwischen 1995 und 2015 durchgeführt wurden. Nach Überprüfung aller Einschluss - und Ausschlusskriterien blieben insgesamt noch 19 potentielle Studien für dieses Review übrig.

Zusammenfassung: Die Übersichtsarbeit erlaubt die Vermutung, dass unsichere Bindungsmechanismen eine Rolle spielen in der Entwicklung 
und Aufrechterhaltung von Schmerzzuständen. Unsichere Bindung wurde mit höheren Depressionswerten, vermehrter Katastrophisierung, erhöhte Opioideinnahme, einem schlechteren Umgang mit dem Schmerz, weniger Compliance mit Behandlungen und insgesamt einem schlechteren Therapieerfolg in Zusammenhang gebracht. Demnach ist eine Berücksichtigung interaktioneller Motive von Patienten wichtig, um das Vertrauen in die Behandlung zu stärken und die Compliance und Veränderungsbereitschaft auf Seitens des Patienten zu erhöhen. Es ist außerdem hilfreich, mit den Patienten zu erarbeiten, dass und inwiefern die erlebte körperliche Symptomatik mit psychologischen Faktoren und Motiven, wie etwa Bindungsstilen, in einem Zusammenhang steht. Ein Arbeitsbündnis zwischen Therapeut und Patient entsteht umso leichter, wenn der Therapeut als zuverlässige und vertrauenswürdige Bindungsperson auftritt.

\section{P10.06 - Einmalige Konditionierung macht aus neutralen Adjektiven negative Konotation}

\section{T. Weiss, W. Miltner}

Institut für Psychologie, FSU Jena, Biologische und Klinische Psychologie Jena, Deutschland

Chronische Schmerzpatienten verarbeiten schmerzassoziierte Worte anders als gesunde Versuchspersonen. Da diese Differenz nicht angeboren sein kann, müssen diese veränderte Verarbeitung im Laufe der Zeit des chronischen Schmerzleidens erlernt werden. Auf der Grundlage dieser Überlegungen und vorangegangener Experimente (Montoya et al, 1996; Miltner et al,1999) wurde die Valenzverarbeitung neutrale Adjektive während der Konditionierung mit schmerzhaften elektrische Reize sowie die folgende Extinktion mit der Frage untersucht, ob bereits eine einmalige Kopplung der Adjektive mit Schmerzreizen ausreicht, um die Valenz dieser Adjektive in Richtung Schmerz zu beeinflussen.

Zur Prüfung dieser Hypothese wurden 37 Personen untersucht. Den Probanden wurden 40 zu lernende neutrale Adjektive präsentiert, die im darauffolgenden Konditionierungsdurchgang erneut gezeigt wurden, wobei jeweils die Hälfte mit bzw. ohne Schmerzreizen präsentiert wurde. Die 2 Konditionierungssets wurden den Probanden abwechselnd zugeordnet. Es sollte die Valenz der Wörter anhand einer 9-stufigen Skala ( $1=$ sehr positiv, $5=$ neutral, $9=$ sehr negativ) eingeschätzt werden. Es folgten vier Extinktionsdurchgänge, in denen jeweils zu den bekannten 40 Adjektiven 40 neue hinzugefügt wurden. Dabei mussten die Probanden entscheiden, ob das gezeigte Wort zum Set der gelernten Adjektive gehörte. Nach Abschluss der Erhebung folgte ein unerwarteter Gedächtnistest, bei dem die Probanden 90 Sekunden Zeit hatten, um die spontan erinnerten Wörter aus dem Lerndurchgang aufzuschreiben (Free-Recall-Phase).

In der Verhaltensanalyse wurde für jede VP über die relevanten Phasen des Experiments (Lernen, Konditionierung, Extinktion 1-4) hinweg jeweils die mittlere Valenz der konditionierten und der unkonditionierten Adjektive mit Hilfe einer zweifaktoriellen Varianzanalyse mit Messwiederholung auf den beiden Faktoren Wortset und Experimentalphase ausgewertet. Außerdem wurden die mittleren Wiedererkennungsleistungen zwischen den Sets (konditionierte vs. unkonditionierte Adjektive) und die Erinnerungsleistung aus der Free-Recall-Phase verglichen.

Innerhalb der Konditionierungsphase wurde signifikanter Valenzunterschied zwischen den konditionierten und unkonditionierten Adjektiven gefunden. Bei den Wiederekennens- und Erinnerungsleistungen zeigten sich keine signifikanten Unterschiede zwischen den beiden Sets.

Die Ergebnisse zeigen, dass die Valenzbewertung von neutralen Adjektiven bereits bei einmaliger Konditionierung mit einem Schmerzreiz nachhaltig beeinflusst wird.

\section{P10.07 - Der Einfluss von Persönlichkeitsvariablen auf die individuelle Schmerzempfindlichkeit}

M. Berkemann', J. Wirth', F. Geis', F. Henrich'², R. Treede', W. Magerl' ${ }^{1}$ Ruprecht-Karls-Universität Heidelberg, Lehrstuhl für Neurophysiologie, Zentrum für Biomedizin und Medizintechnik Mannheim, Mannheim, Deutschland, ${ }^{2}$ Medizinische Fakultät Mannheim der Universität Heidelberg, Mannheim, Lehrstuhl für Neurophysiologie, Zentrum für Biomedizin und Medizintechnik Mannheim, Mannheim, Deutschland, ${ }^{3}$ Universität Heidelberg, Lehrstuhl für Neuropyhsiologie, Mannheim, Deutschland, ${ }^{4}$ Medizinische Fakultät Mannheim, Lehrstuhl für Neurophysiologie, CBTM, Mannheim, Deutschland

Ziel der Untersuchung: Ein Einfluss von Persönlichkeitsvariablen auf Schmerzempfindlichkeit und Schmerzkontrolle sind in vielen Untersuchungen an Patienten dokumentiert. An gesunden Versuchspersonen ist das bisher nicht an hinreichend großen Kohorten von Versuchspersonen untersucht. Wir haben den Einfluss von Persönlichkeitsvariablen auf eine Schmerzschwelle, Schmerzratings und endogenen Schmerzkontrolle (Conditioned pain modulation CPM) an jungen gesunden Versuchspersonen untersucht.

Methoden: 231 gesunde Medizinstudenten des 2. Studienjahrs (116 männlich, 115 weiblich, Alter 22,3 $\pm 3,3$ Jahre) wurden untersucht auf den Einfluss von Persönlichkeitseigenschaften auf die Schmerzempfindlichkeit. Es wurde ein Schmerzschwellenmaß (Druckschmerzschwelle am linken Handballen, Wagner Instruments Druckalgometer, $1 \mathrm{~cm}^{2}$ Kontaktfläche, Reizanstieg $50 \mathrm{kPa} / \mathrm{s}$ ) eingesetzt. Außerdem wurde ein tonischer überschwelliger Schmerzreiz verwandt (Eiswasserbad der rechten Hand bis zum Handgelenk für bis zu max. $3 \mathrm{~min}=$ Cold Pressor Test, Rating auf einer 0 - 100 NRS). Die schmerzinduzierte Schmerzsuppression durch den Cold Pressor Test induziert (Vergleich Druckschmerzschwellen vor und nach CPM).

Die Probanden füllten online verschiedene Fragebögen: Deutsche Version der Perceived Stress Scale (PSS), Skalen für Verhaltensinhibition und Verhaltensaktivierung (Behavioural Inhibition - Behavioural Activation Scales BIS-BAS), eine Schmerzkatasthrophisierungsskala (Pain Catastrophising Scale PCS), die Trait-Subskalen des Spielberger State-Trait Anxiety Index (STAIX), Saarbrücker Persönlichkeitsfragebogen (SPF, Deutsche Version des Interpersonality Reactivity Index IRI), und Erhebung der „Big Five“ Persönlichkeitsdimensionen (NEO FFI). Zusätzlich schätzten die Versuchspersonen ihren momentanen Stresslevel auf einer 0 - 100 NRS. Ergebnisse: Die weiblichen Studenten hatten in fast allen Skalen für Stress, Neurotizismus, Schmerzkatastrophisierung etc. signifikant höhere Scores (vergleichbar der Eichstichprobe). Die einzige Traitvariable, die mit der Druckschmerzschwelle schwach korrelierte, war Schmerzkatastrophisierung $(r=-0,15, p<0,05)$. Im Gegensatz dazu bestanden zahlreiche signifikante Korrelationen der Persönlichkeitsvariablen zu Schmerzratings im tonischen Kälteschmerz. Die Kälteschmerzratings korrelierten mit der Schmerzkatastrophisierung $(\mathrm{r}=0,30, p<0,001$; Partialkorrelation $\mathrm{r}=0,43$, wenn die signifikante Varianz des Körpergewichts berücksichtigt wurde), mit der Verhaltensinhibition BIS ( $\mathrm{r}=0,24, p<0,001)$, Neurotizismus $(\mathrm{r}=0,18, p<0,005)$, und der Personal Distress Subscale der IRI Empathieskala $(r=0,41, p<0,001)$. Das subjektiv wahrgenommene Stresslevel wies die höchste Korrelation auf $(\mathrm{r}=0,62, p<0,001)$. Versuchspersonen, die den Cold Pressor Test vor Ende der max. Exposition von $180 \mathrm{~s}$ wegen Erreichen der Schmerztoleranz abbrachen, hatten signifikant höhere Scores für Schmerzkatastrophisierung, Irritierbarkeit und Ängstlichkeit (alle $p<0,05$ ). Wir konnten keinerlei signifikante Beziehungen zwischen den erhobenen Persönlichkeitsvariablen und der Schmerzhemmung identifizieren.

Schlussfolgerung: Persönlichkeitsvariablen zeigen einen geringen Einfluss auf die Druckschmerzschwelle, aber einen deutlichen Einfluss auf den überschwelligen tonischer Kälteschmerz. Es bestanden keine Beziehungen zu CPM. 


\section{P11 - Rückenschmerz und Bewegungsapparat/ Schmerz und Alter/Tumorschmerz und Palliativmedizin}

\section{P11.01 - Ergebnisse eines Case-Management Projekts zur Verbesserung der Behandlung von chronischen Rückenschmerzen}

M. Mayer', W. Besier ${ }^{1}$, M. Dusch ${ }^{2}$, M. Steinisch ${ }^{3}$, R. Herr ${ }^{3}$, J. Fischer ${ }^{3}$, R. Treede ${ }^{4}$

${ }^{1} G$ enossenschaft Gesundheitsprojekt Mannheim e G, Mannheim, Deutschland, ${ }^{2}$ Medizinische Fakultät Mannheim, Universität Heidelberg, Klinik für Anästhesiologie und Operative Intensivmedizin, Mannheim, Deutschland, ${ }^{3}$ Medizinische Fakultät Mannheim der Universität Heidelberg, Mannheimer Institut für Public Health, Sozial- und Präventivmedizin, Mannheim, Deutschland, ${ }^{4}$ Universität Heidelberg, Lehrstuhl für Neuropyhsiologie, Mannheim, Deutschland

Hintergrund: Knapp ein Viertel der Gesamtbevölkerung in Deutschland leidet unter chronischen Schmerzen. Bei der Therapie von Patienten mit chronischen Schmerzen ist es von besonderer Bedeutung, die Behandlungsstrategie nicht nur an die Schmerzsymptomatik sondern auch an die Lebensumstände und Begleiterkrankungen des Patienten anzupassen. Case Management (CM) ermöglicht die Entwicklung eines Behandlungsplans unter Berücksichtigung des spezifischen Lebenskontexts des Patienten sowie einer gezielten Koordination und Planung von Behandlungsabläufen. Ziel dieser Studie war es, den Effekt von CM auf die Behandlungserfolge bei Patienten mit chronischen Rückenschmerzen zu überprüfen.

Methode: Eine randomisierte kontrollierte Studie untersuchte den Einfluss von CM auf die Beurteilung verschiedener Schmerzwahrnehmungen (Keele STarT Back Screening Tool; Pain Catastrophizing Scale; SES Schmerzempfindungsskala; Einzelitems aus dem Pain detect Fragebogen [augenblicklicher Schmerz; stärkste Schmerz in den letzten 4 Wochen; durchschnittliche Schmerz in letzten 4 Wochen]) sowie der physischen und psychischen Gesundheit (SF 12; Depression; Ängstlichkeit). Dazu wurden in 33 Praxen im Raum Mannheim Patienten mit chronischen Rückenschmerzen zufällig in die Behandlungsgruppe ( $n=60$; mit CM) und die Gruppe mit Regelversorgung ( $n=60$; ohne $\mathrm{CM})$ eingeteilt. Die Erhebung erfolgte zu zwei Messzeitpunkten (Baseline: Januar-Juni 2014; Follow-up: August-Juni 2015). Die Intervention umfasste ein aufsuchendes CM mit einer Interventionsintensität von zwei Besuchen/Monat im ersten halben Jahr und einem Besuch/Monat im zweiten halben Jahr. Die Daten wurden über wellen- und behandlungsspezifische Mittelwerte imputiert und mittels Mixed Effects Modellen (fixed effects: CM, Welle; random effects: Praxis, Patient) mit Interaktion für Welle $\times \mathrm{CM}$ analysiert.

Ergebnisse: Die Teilnehmer waren zu $65 \%$ weiblich, im Schnitt 64 Jahre alt und hatten einen durchschnittlichen BMI von $29 \mathrm{~kg} / \mathrm{m}^{2} .36 \%$ hatten einen Reha-Aufenthalt in den letzten 4 Jahren. Zur Baseline Erhebung gab es keine signifikanten Gruppenunterschiede. Hinsichtlich des CM zeigte sich lediglich in der Bewertung der Schmerzstärke in den letzten 4 Wochen ein signifikanter Effekt (mittlere Differenz $\mathrm{CM}=0,94 \pm 0,65$; Kontrolle $=-0,13 \pm 0,51 ; P=0,025)$. Generell legt eine grafische Exploration jedoch mögliche Subgruppeneffekte nahe. Dies bedeutet, dass das CM möglicherweise nur einen Effekt in einem spezifischen Teil der Behandlungsgruppe hatte.

Schlussfolgerung: Diese Studie konnte keinen eindeutigen Effekt von CM auf die verschiedenen Schmerzwahrnehmungen und Gesundheitsindizes zeigen. Eine mögliche Erklärung mag in dem Vorhandensein von spezifischen Subgruppen liegen: eine Gruppe in welcher CM weniger wirkt und eine weitere Gruppe in welcher CM eine Wirkung zeigt. Die Identifikation solch vulnerablen Gruppen stellt ein zukünftiges Ziel dar.

Förderung: Der Beitrag wird unterstützt von folgenden Institutionen: Mundipharma $\mathrm{GmbH}$
P11.02

\section{Aktivierende Schmerztherapie - in Ihrem Alter?! - Multimodale Schmerztherapie bei Senioren}

\section{E. Sens, M. Mothes-Lasch, K. Große, B. Otto, J. Lutz}

Zentralklinik Bad Berka GmbH, Zentrum für Interdisziplinäre Schmerztherapie, Bad Berka, Deutschland

Aufgrund der demographischen Entwicklung, mit zunehmender Nachfrage altersangepasster Therapieangebote, wurde auch im Zentrum für interdisziplinäre Schmerztherapie in Bad Berka eine stationäre multimodale Schmerztherapie (MMST) mit Senioren eingerichtet. Hierfür erfolgte eine Anpassung des bestehenden dreiwöchigen Programms (gemäß OPS 8-918,14) mit Kürzung der Vortrags- und Seminardauer (je 60 Minuten), Erhöhung der Anzahl physiotherapeutischer Einzeltherapien sowie Verringerung der Gruppengröße (4 bis 6 Patienten). Dennoch erfordert die altersangepasste MMST im Zentrum für Interdisziplinäre Schmerztherapie (ZIST) von den Patienten eine hohe Motivation zur aktiven Mitarbeit sowie die Bereitschaft, sich von passiver Behandlungserwartung abzuwenden und auf neue Ansätze einzulassen.

Von August 2014 bis Juni 2016 haben im ZIST 96 Patienten (66,7\% wbl.) an der dreiwöchigen MMST teilgenommen. Die Altersverteilung der Senioren lag zwischen 57 und 86 Jahren, wodurch deutlich wird, dass der Begriff „Senioren“ keine fest definierte Altersklasse darstellt. Ein Anteil von $45 \%$ der Patienten war zum Aufnahmezeitpunkt auf Opiatanalgetika eingestellt. Während der Therapie beendeten davon $77 \%$ diese Langzeittherapie vollständig, alle anderen Patienten erreichten eine Dosisreduktion. Die Analyse der erhobenen psychometrischen Parameter (t-Test, ANOVA mit Messwiederholung) ergab eine wesentliche Verbesserung der Lebensqualität sowie eine Verringerung der Schmerzstärke, der Depressivität, der Ängstlichkeit und der subjektiven Stressbelastung. Auch die Betrachtung der Katamnese deutet auf nachhaltige Effekte bei den erhobenen Parametern mit Ausnahme der Schmerzstärke und der Ängstlichkeit hin.

Zusammenfassend zeigen unsere Ergebnisse, dass eine Anpassung der multimodalen Therapie für Senioren erfolgreich möglich ist und langfristig zu einer Verbesserung der Lebensqualität führen kann.

\section{P11.03 - Zu alt für eine Therapie?! - Ergebnisse eines altersangepassten 3-wöchigen multimodalen Therapieprogrammes}

B. Oehme, U. Thöner, M. Mothes-Lasch, E. Sens, J. Lutz

Zentralklinik Bad Berka GmbH, Zentrum für Interdisziplinäre Schmerztherapie, Bad Berka, Deutschland

Multimodale Schmerztherapie und die darin integrierte aktivierende Bewegungstherapie erwies sich in bisherigen Untersuchungen als sehr gut geeignete Methode zur Behandlung chronischer Schmerzpatienten. Aus der Erfahrung gemischter Therapiegruppen mit jüngeren und älteren bzw. mobilitätseingeschränkten Schmerzpatienten kristallisierte sich jedoch die Notwendigkeit eines spezifisch altersangepassten multimodalen Therapiekonzeptes heraus.

Seit August 2014 wurden im Zentrum für interdisziplinäre Schmerztherapie in Bad Berka (ZIST) insgesamt 96 Patienten im Rahmen eines stationären 19-tägigen multimodalen Therapieprogrammes behandelt, wobei die Patienten überwiegend hochchronifiziert (MPSS III 96\%) und hochbeeinträchtigt (von Korff IV $68 \%$ ) waren. Ziele der physiotherapeutischen Behandlung sind der Erhalt der persönlichen Selbständigkeit und Mobilität, der Abbau von Sturzangst und Vermeidungsverhalten sowie die Verringerung des Risikos einer Pflegebedürftigkeit.

Die Analyse (t-Test, ANOVA mit Messwiederholung) der erhobenen funktionellen physiotherapeutischen Tests und der Selbstbeurteilungsskala FFbH-R ergaben signifikante Verbesserungen zwischen Therapiebeginn und Therapieende. Der Timed ,up\&go" (Podsiadlo \& Richardson) ergab eine Verbesserung der Mobilität (von 16,96 sec auf 11,49 sec), der Motilitätstests nach Tinetti zeigte eine Steigerung der Bewegungssicherheit (von 17,46 Punkte auf 24,28 Punkte) und die Back performance scale (nach Myklebust) wies eine Abnahme der Beeinträchtigung von Aktivitä- 
ten (von 8,22 Punkte auf 4,98 Punkte) auf. Im Funktionsfragebogen Hannover zur alltagsnahen Diagnostik der Funktionsbeeinträchtigung durch Rückenschmerzen (FFbH-R, Kohlmann \& Raspe) zeigte sich im Mittel eine signifikante Verbesserung von $38,17 \%$ zu Therapieanfang auf $54,84 \%$ zu Therapieende. Bezüglich der Katamnese nach 8 Monaten ergab sich für die Werte des FFbH-R jedoch eine Reduktion auf 44,62\%.

Ein altersangepasster stationärer multimodaler Behandlungsansatz über 3 Wochen bei Patienten im Seniorenalter bzw. mit eingeschränkter Mobilität führt nach Therapieende zu einer deutlichen Verbesserung in allen funktionellen Bereichen, einer Erhaltung und Steigerung der körperlichen Aktivität, einer Verminderung des Risikos von Stürzen sowie einer Stärkung des Vertrauens in die eigene körperliche Leistungsfähigkeit. Eine stationär multimodale Therapie bei hochchronifizierten Seniorenpatienten ist erfolgreich möglich. Aufgrund des Fehlens einer Langzeitbeobachtung der funktionellen physiotherapeutischen Tests über das Therapieende hinaus, kann keine Aussage über die Langfristigkeit der Erfolge gemacht werden. Jedoch impliziert die Verringerung der Werte des FFbH-R, dass eine qualifizierte Nachbetreuung am Wohnort oder eine geplante Therapieauffrischung nach Abschluss des intensiven multimodalen Therapieprogrammes förderlich sein könnte.

\section{P11.04 - Schmerzassessment bei Delir: Eine Scopingreview}

\section{T. Fischer1, M. Agar ${ }^{2}$, A. Hosie 2 , T. Luckett ${ }^{2}$, J. Phillips ${ }^{2}$}

'Evangelische Hochschule Dresden, Pflegewissenschaft, Dresden, Deutschland, ${ }^{2}$ University of Technology Sydney, Faculty of Health, Centre for Cardiovascular and Chronic Care, Ultimo, Australien

Hintergrund: Delire sind eine neurokognitive Störung mit multifaktorieller Aetiologie. Sie sind durch Störungen der Aufmerksamkeit und des Bewusstseins gegkennzeichnet, die innerhalb kurzer Zeit entstehen und oftmals flukturieren (APA 2013). Delire treten besonders häufig bei älteren Menschen, postoperativ oder in Palliativsituationen auf, teilweise mit Prävalenzraten von über $80 \%$. In diesen Patientengruppen ist gleichzeitig mit einer hohen Schmerzprävalenz zu rechnen. Delire erschweren das Schmerzassessment oder machen es sogar unmöglich, weil die Betroffenen nicht oder nur eigeschränkt Auskunft zu Schmerzen geben können. Da die Delirsymptomatik sich zum Teil mit typischem Schmerzverhalten bei Demenz überschneidet, können Instrumente für das Schmerzassessment bei Demenz nicht einfach übernommen werden. Dennoch fehlt es bislang an klinischen Ansätzen und Forschung zu diesem Themengebiet. Ziel dieser Scopingreview war es daher, den Umfang der Strategien, Instrumente, Ansätze, Konzepte und wissenschaftlichen Ergebnisse zum Schmerzassessment bei Deliren in unterschiedlichen Settings darzustellen. Methode: Die Scopingreview folgte der Methodologie des Joanna Briggs Institute (JBI) für Scoping Reviews (JBI 2015). Es wurde eine dreistufige Suchgstrategie angewandt: 1. Begrenzte Suche mit den MeSH-Terms bzw. Major Headings „Delirium“ und „Pain Measurement" in den Datenbanken Medline und CINAHL. 2. Anhand der Ergebnisse wurde die Suchanfrage verfeinert und in den Datenbanken Medline, CINAHL, Scopus, Embase sowie Proquest durchgeführt. 3. Auswertung von Literaturlisten und ggf. Nachfrage bei Autoren.

Titel und Abstracts der Treffer wurden anhand vorab festgelegten Kriterien auf Relevanz für die Fragestellung geprüft. Die eingeschlossenen Quellen wurden schliesslich anhand vorab entwickelter forschungsbezogener und klinischer Kriterien analysiert.

Ergebnisse: Die verfeinerte Suche in allen Datenbanken ergab 1396 Treffer. Davon erwies sich ein Grossteil als nicht relevant so dass nur eine geringe Anzahl an Quellen anhand von Publikationsart, Population, Setting, Art des Deliriums, Schmerzassessmentstrategie, Schmerzassmenntinstrument sowie Studienergebnissen ausgewertet wird. Die Ergebnisse werden Auskunft darüber geben, welche Strategien zum Schmerzassessment bei Delir angewandt werden, welche Forschungsansätze dabei bislang verfolgt wurden und welche Ergebnisse vorliegen.

Diskussion: Obwohl zahlreiche Patienten und insbesondere ältere Menschen von Deliren betroffen sind, fehlt es bislang an Forschungsergebnissen und klinischen Ansätzen, um Schmerzen in dieser Patientengrup- pe einzuschätzen. Ob Instrumente geeignet sind, die für andere kognitive Einschränkungen (vor allem Demenzen) entwickelt wurden, ist fraglich, weil Delirsymptome Schmerzverhalten sich teils überschneiden. Das Ergebnis dieser Review trägt dazu bei, den Wissensstand zum Schmerzassessment bei Delir zu systematisieren, Lücken zu identifizieren und damit Forschung und Entwicklungsansätze zu leiten.

\section{P11.05 - Schmerz-Komorbidität bei Restless-Legs-Syndrom - Argumente für eine frühzeitige Opioidtherapie?}

\section{J. Kohler}

Zentrum für Neurologie \& Neurogeriatrie, Emmendingen, Deutschland

Einleitung: Das Restless-Legs-Syndrom (RLS) ist eine der häufigsten neurologischen Erkrankungen. Therapie der ersten Wahl sind L-Dopa oder Dopaminagonisten (DA). Dabei ist jedoch der Wirkverlust (Augmentation) unter dieser Behandlung ein ernst zu nehmendes therapeutisches Problem im klinischen Alltag. Gleichzeitig sind chronische Schmerzen unterschiedlichster Ätiologie in einer alternden Bevölkerung sehr häufig. Eine Komorbidität eines RLS mit einem chronischen Schmerzsyndrom ist deshalb in ärztlicher Praxis häufig. Die aktuellen Leitlinienempfehlungen der Deutschen Gesellschaft für Neurologie zur Behandlung des RLS mit Präferenz einer dopaminergen Therapie müssen in diesen Fällen hinterfragt werden.

Patienten: Zwischen Januar und Dezember 2015 haben wir 33 Patienten (PAT), 27 Frauen, Durchschnittsalter 70 (range 46-93) Jahre, mit klinisch gesichertem RLS hinsichtlich eines komorbiden chronischen Schmerzsyndroms bezüglich der angewandten therapeutischen Strategien und deren Effektivität in unserem Zentrum evaluiert. Ergebnisse: 27/33 PAT wurden bezüglich des RLS ausschließlich mit L-Dopa oder einem DA behandelt. 4/33 PAT erhielten wegen Unverträglichkeit oder Unwirksamkeit der dopaminergen Medikation eine Monotherapie mit einem Opioid (Oxycodon/Naloxon oder Tilidin/Naloxon). 21/33 PAT klagten gleichzeitig über alltagsrelevante chronische Schmerzen unabhängig vom RLS.

Hierbei handelte es sich überwiegend um chronische nozizeptive oder gemischt neuropathisch-nozizeptive Schmerzen, meistens vor dem Hintergrund einer Osteoarthritis. 7/21 PAT erhielten eine Kombination aus dopaminerger Medikation und Opioidtherapie und bei 8/21 PAT wurde Pregabalin (PGL) ergänzend eingesetzt. Bei allen behandlungsbedürftigen PAT konnte durch eine individualisierte Medikation eine gute bis sehr gute Symptomkontrolle sowohl des RLS als auch der Schmerzen erzielt werden. Schlussfolgerung: In unserem kleinen Kollektiv fand sich bei $2 / 3$ der überwiegend alten behandlungsbedürftigen PAT mit RLS eine Komorbidität mit einem chronischen Schmerzsyndrom. Diese profitierten durchweg von einer Komedikation mit einem Opioid oder PGL. Bei der Behandlung des RLS sollten zukünftig komorbide Schmerzen besser in die medikamentösen Behandlungsentscheidungen integriert werden. Sowohl Opioide, insbesondere das zugelassene Oxycodon/Naloxon, als auch PGL, derzeit noch off label, sollten hier einen größeren Stellenwert in der individualisierten Therapie erhalten, zumal das Risiko einer Augmentation unter diesen Behandlungen geringer zu sein scheint.

\section{P11.06 - Mobilität und Schmerz: Gibt es Unterschiede bei Bewohnern in Altenhilfeeinrichtungen?}

N. Nestler', L. Krisch', A. Mahlknecht ${ }^{2}$, M. Flamm², J. Osterbrink ${ }^{1}$

'Paracelsus Medizinische Privatuniversität, Institut für Pflegewissenschaft und -praxis, Salzburg, Österreich, ${ }^{2}$ Paracelsus Medizinische Privatuniversität, Institut für Allgemein-, Familien- und Präventivmedizin, Salzburg, Österreich

Hintergrund: Viele Bewohner in Altenhilfeeinrichtungen haben Schmerzen [1]. Häufig geht eine Mobilitätseinschränkung mit Schmerz einher und mindert die Selbständigkeit der Betroffenen. In einem Projekt zur Untersuchung der Arzneimitteltherapiesicherheit in Altenhilfeeinrichtungen wurde als Subgruppenanalyse untersucht, ob und wie sich Schmerzpatienten von Nicht-Schmerzpatienten hinsichtlich der vorhandenen Diagnosegruppen, der Anzahl der Medikamente und der Mobilität unterscheiden. 
Methode: Bewohner in Altenhilfeeinrichtungen wurden mit der Verbalen Rang Skala zum Vorhandensein von Schmerzen befragt. Bei kognitiv beeinträchtigten Bewohnern wurde zusätzlich das Schmerzverhalten mittels BESD erhoben. Mit dem Time-Up-and-Go Test wurde die Mobilität erfasst, die verordneten Medikamente wie auch die Diagnosen der Pflegedokumentation entnommen.

Ergebnisse: Es konnten Daten von 108 Bewohnern in die Auswertung einbezogen werden. Bewohner mit Schmerzen hatten häufiger eine COPD, Herzinsuffizienz, Diabetes Mellitus, Depressionen, Chronische Niereninsuffizienz oder kardiale Herzrhythmusstörungen als Bewohner ohne Schmerzen. Die durchschnittliche Anzahl aller Medikamente war bei den Bewohnern mit Schmerz ( $M W=12,2, S D=5,2)$ höher als bei den Bewohnern ohne Schmerz (MW $=10, S D=4,4)$. Die Mobilität der Bewohner mit Belastungsschmerz war eingeschränkter, nur 8,8\% der Bewohner hatten eine geringe Mobilitätseinschränkung (33,3\% der Bewohner ohne Schmerz). Über $50 \%$ der Bewohner mit geringer Mobilitätseinschränkung hatten keinen Belastungsschmerz. Von den Bewohnern mit einer ausgeprägten Einschränkung gaben 51,8\% mittlere bis starke Schmerzen an, 22,2\% hatten keine Belastungsschmerzen. Auch bei kognitiv beeinträchtigten Bewohnern zeigten sich mehr Mobilitätseinschränkungen in der Gruppe der Personen mit Schmerzverhalten bei Belastung, als bei jenen ohne Anzeichen für Schmerz. Diskussion: Schmerz und eingeschränkte Mobilität sind zwei Phänomene, die gemeinsam auftreten. Vorhandene Belastungsschmerzen scheinen dabei insbesondere mit Einschränkungen in der Mobilität einherzugehen. Eine häufigere Medikamenteneinnahme konnte für die Schmerzpatienten festgestellt werden, jedoch fehlen Daten, ob auch die vermehrte Medikamenteneinnahme bzw. die Multimorbidität im Zusammenhang zur eingeschränkten Mobilität stehen. Hier sollten weitere Untersuchungen durchgeführt werden, um die Bedeutung der Medikation für die Mobilität und damit die Selbständigkeit der Bewohner weiter herausarbeiten zu können.

\section{Literatur}

1. Osterbrink J, Hufnagel M, Kutschar P, Mitterlehner B, Krüger C, Bauer Z, Aschauer W, Weichbold M, Sirsch E, Drebenstedt C, Perrar KM, Ewers A (2012) Die Schmerzsituation von Bewohnern in der stationären Altenhilfe. Schmerz 26(1): 27-35

\section{P11.07 - Einfluss von Paracetamol, Metamizol und 4-N-Methylaminoantipyrin auf Pankreaskarzinomzellen in vitro}

\section{A. Bundscherer', M. Malsy', C. Lassen ${ }^{1}$, T. Rost', B. Graf ${ }^{2}$}

'Universitätsklinikum Regensburg, Klinik für Anästhesiologie, Regensburg, Deutschland, ${ }^{2}$ Universitätsklinikum Regensburg, Klinik für Anästhesiologie, Regensburg, Deutschland

Zielsetzung: Es gibt Hinweise darauf, dass die perioperative Periode eine vulnerable Phase darstellt, in der Tumorprogression und Metastasierung begünstigt werden (Ben-Eliyahu, 2003; Gottschalk et al., 2010). Die Kombination aus chirurgischer Manipulation und perioperativer Kompromittierung der Immunkompetenz erhöht das Risiko einer Tumordissemination und kann sich negativ auf den Krankheitsverlauf onkologischer Patienten auswirken (Bundscherer et al., 2014; Goldfarb and Ben-Eliyahu, 2006). Welchen Einfluss perioperativ eingesetzte Analgetika und Anästhetika auf die Tumorprogression haben ist weithin unklar. Im Rahmen der perioperativen Schmerztherapie werden üblicherweise Kombinationen aus Opioidanalgetika und Nicht-Opioid- Analgetika wie Metamizol und Paracetamol angewandt (Pogatzki-Zahn et al., 2014). Ziel dieser Studie war es, den Einfluss von Paracetamol, Metamizol und dessen aktiven Metaboliten MAA (4-N-Methylaminoantipyrin) auf Proliferation und Apoptoserate von Pankreaskarzinomzellen (PANC 1 und PaTu8988 t) in vitro zu untersuchen.

Methodik: Die Analyse der Zellproliferation wurde mit Hilfe des Cell Proliferation ELISA BrdU Assays (Roche) durchgeführt. Hierzu wurden PANC 1 und PaTu8988 t Pankreaskarzinomzellen für 48 h mit 1-500 $\mu \mathrm{M}$ Paracetamol, Metamizol oder MAA inkubiert. Nach Inkubation der Zellen mit $250 \mu \mathrm{M}$ der Testsubstanzen für 3-24 h wurde die Apoptoserate nach Doppelfärbung mit Annexin V und Propidium Jodid (BD Pharming) durchflusszytometrisch erfasst.
Ergebnis: Metamizol oder MAA führten in PANC 1 Pankreaskarzinomzellen zu keiner Veränderung der Apoptoserate. In PaTu8988 t reduzierten Metamizol und MAA die Apoptoserate nach $3 \mathrm{~h}$ Inkubationszeit signifikant, nach $9 \mathrm{~h}$ Inkubationszeit konnte eine Steigerung der Apoptoserate durch Metamizol beobachtet werden. $\mathrm{Zu}$ den anderen Inkubationszeitpunkten wurden keine Veränderungen durch Metamizol oder MAA detektiert. Paracetamol hatte bei beiden Pankreaskarzinomzellen keinen Einfluss auf die Apoptose. In einer Dosierung von 1-250 $\mu \mathrm{M}$ reduzierte Metamizol die Zellproliferation in beiden Pankreaskarzinomzellen, während der aktive Metabolit MAA keine antiproliferative Effekte entfaltet. Paracetamol hemmte das Zellwachstum in PaTu8988 t Zellen in allen Konzentrationen, während in PANC 1 Zellen nur $1 \mu \mathrm{M}$ Paracetamol antiproliferativ wirkte.

Schlussfolgerung: Die Ergebnisse dieser Studie deuten darauf hin, dass klinisch erreichbare Konzentrationen von Paracetamol keine, Metamizol und dessen aktiver Metabolit MAA nur geringen Einfluss auf die Apoptose von Pankreaskarzinomzellen hat. Paracetamol und Metamizol, nicht aber MAA hatten Einfluss auf das Zellwachstum. Weitere Studien mit unterschiedlichen Tumorentitäten sind notwendig, um zu beurteilen, ob eine perioperative Schmerztherapie mit Metamizol oder Paracetamol die Tumorprogression beeinflussen kann.

\section{Literatur}

Ben-Eliyahu S (2003) The promotion of tumor metastasis by surgery and stress: immunological basis and implications for psychoneuroimmunology. Brain Behav Immun 17(Suppl 1):S27-36.

Bundscherer A, Malsy M, Bitzinger D, Graf BM (2014) [Interaction of anesthetics and analgesics with tumor cells]. Der Anaesthesist 63:313-315.

Goldfarb Y, Ben-Eliyahu S (2006) Surgery as a risk factor for breast cancer recurrence and metastasis: mediating mechanisms and clinical prophylactic approaches. Breast disease 26:99-114.

Gottschalk A, Sharma S, Ford J, Durieux ME, Tiouririne M (2010) Review article: the role of the perioperative period in recurrence after cancer surgery. Anesthesia and analgesia 110:1636-1643.

Pogatzki-Zahn E, Chandrasena C, Schug SA (2014) Nonopioid analgesics for postoperative pain management. Current opinion in anaesthesiology 27:513-519

\section{P12 - Schmerz bei Kindern}

\section{P12.01 - Störungsspezifische Testdiagnostik auf dem Prüfstand: Vorläufige Ergebnisse eines Screening-Tools für Traumafolgestörungen bei Kindern mit stark beeinträchtigenden chronischen Schmerzen}

V. Lissek, F. Flack, B. Zernikow, J. Wager

Deutsches Kinderschmerzzentrum, Vestische Kinderklinik, Universität Witten/ Herdecke, Datteln, Deutschland

Hintergrund: Studien zeigen einen negativen Einfluss von Stress und traumatisierenden Ereignissen auf das Schmerzerleben. In der Anamnese chronisch schmerzkranker Kinder und Jugendlicher wird häufig ein traumatisches Ereignis benannt oder es lassen sich die Symptome einer Posttraumatischen Belastungsstörung (PTBS) beobachten. Geeignete Screeningverfahren sind notwendig, um frühzeitig das Vorhandensein von Symptomen einer PTBS zu erkennen. Ein gängiges Instrument zum PTBS-Screening ist die von Ricky Greenwald entwickelte „Child Report of Post-Traumatic Symptoms Scale“ (CROPS), die auch deutschsprachig vorliegt. Im CROPS beurteilen die Kinder und Jugendlichen im Selbstbericht das Zutreffen von Aussagen zu posttraumatischen Belastungssymptomen. Ziel der aktuellen Studie ist die Überprüfung des CROPS bei Kindern und Jugendlichen mit chronischen Schmerzen. Methode: Bei Aufnahme zu einer 3- bis 4- wöchigen stationären interdisziplinären Schmerztherapie füllen Kinder und Jugendliche (7-17 Jahre) den CROPS aus (Wertebereich: 0 - 50; Cut-off Wert $>19=$ klinisch auffällig). Im CROPS beurteilen die Kinder und Jugendlichen im Selbstbericht das Zutreffen von Aussagen posttraumatischer Belastungssymptome. Zudem erfolgt die Diagnosevergabe einer PTBS durch den behandelnden Therapeuten nach einer streng 
dem DSM-5 entsprechenden kriterienorientierten Diagnose-Checkliste. Die Genauigkeit des vorgegebenen CROPS Cut-Off-Wertes zur Vorhersage der DSM-5 Diagnose wird untersucht. Mithilfe einer ROC-Analyse wird überprüft, ob für das Schmerzsample ein anderer Cut-Off Wert eine bessere Diagnosevorhersage ermöglicht. Insgesamt sollen $N=300$ Kinder und Jugendliche mit chronischen Schmerzen in die Studie eingeschlossen werden. Hier werden erste Zwischenergebnisse präsentiert. Vorläufige Ergebnisse: Bislang wurden $n=100$ Kinder und Jugendliche im Alter von durchschnittlich 15 Jahren $(\mathrm{SD}=1,76)$ rekrutiert. Davon sind 69 Mädchen $(69,0 \%)$ und 31 Jungen $(31,0 \%)$. Im Durchschnitt lag der CROPS Wert bei 17,7 (SD =8,96). Bei $38 \%$ der Kinder und Jugendlichen zeigte sich eine Überschreitung des CROPS Cut-Off-Wertes (>19). Jedoch erfüllten lediglich $13 \%$ der pädiatrischen Schmerzpatienten die Diagnose einer PTBS nach DSM-5. Von den Patienten, die die Kriterien einer PTBS nach DSM-5 erfüllten, überschritten lediglich 54\% den Cut-Off-Wert von 19. Eine ROC-Analyse zeigt, dass die Fläche unter der Kurve (area under curve, AUC) gering ist (AUC $=0,692)$ und somit eine zuverlässige Differenzierung in der bisher untersuchten Patientengruppe mit und ohne PTBS aufgrund der CROPS-Werte nicht möglich ist. Schlussfolgerung: Die vorläufigen Ergebnisse in einer Population von Kindern und Jugendlichen mit stark beeinträchtigenden chronischen Schmerzen geben Hinweise darauf, dass der CROPS nicht zum Screening einer PTBS geeignet ist. Weitere Analysen müssen zeigen, ob eine Anpassung der Skala eine bessere Differenzierbarkeit in einem Schmerzsample ermöglicht.

\section{P12.02 - Wie schwer sind Deine Schmerzen? - Graduierung chronischer Schmerzen als Therapie-Outcome der interdisziplinären Schmerztherapie für Kinder und Jugendliche}

\section{Stah/schmidt, F. Barth, B. Zernikow, J. Wager}

Vestische Kinder- und Jugendklinik Datteln - Universität Witten/Herdecke, Deutsches Kinderschmerzzentrum, Datteln, Deutschland

Hintergrund: Einige Studien konnten bereits zeigen, dass eine interdisziplinäre Schmerztherapie für Kinder und Jugendliche mit chronischen Schmerzen sowohl die Schmerzintensität als auch die Beeinträchtigung im Alltag und schmerzbedingte Schulfehltage reduziert. In dieser Studie sollte das Therapie-Outcome ein Jahr nach ambulanter Erstvorstellung in einer Schmerzambulanz anhand der Graduierung chronischer Schmerzen („Chronic Pain Grading") untersucht werden.

Methode: Die Daten von 267 Jugendlichen wurden bei Erstvorstellung in der Schmerzambulanz und bei einer telefonischen Nachbefragung ein Jahr später erfasst. Nach der Erstvorstellung wurden die Jugendlichen entweder ambulant oder stationär behandelt. Das Chronic Pain Grading (CPG) wurde genutzt, um die Schwere der Schmerzproblematik anhand von Schmerzintensität und schmerzbezogener Beeinträchtigung (zusammengesetzt aus Beeinträchtigung im Alltag und Schulfehltagen) einer von 5 Stufen zuzuordnen $(0=$ keine chronischen Schmerzen, $1=$ geringe Schmerzintensität und geringe Beeinträchtigung, 2 =hohe Schmerzintensität und geringe Beeinträchtigung, 3 =starke schmerzbezogene Beeinträchtigung und mäßige Einschränkung, 4 =starke Beeinträchtigung und starke Einschränkung). Für die Wirksamkeit der Therapie wurde die Veränderung der CPG-Stufen von Erstvorstellung zur Nachbefragung untersucht.

Ergebnisse: Bei Erstvorstellung konnten die wenigsten Patienten (1,6\%) CPG 1 mit geringer Schmerzintensität und geringer Beeinträchtigung zugeordnet werden. Die meisten Patienten $(41,0 \%)$ befanden sich in CPG 3. In CPG 2 und 4 waren jeweils ein Drittel der Patienten (29,1\% bzw. 28,3\%). CPG 0 entfiel bei Erstvorstellung, da die Patienten sich aufgrund chronischer Schmerzen in der Ambulanz vorstellten. Ein Jahr später konnten 23,3\% der Patienten CPG 0 zugeordnet werden, da sie keine Schmerzen mehr berichteten. Die wenigsten Patienten $(4,3 \%)$ befanden sich in CPG 4. Die meisten Patienten (40,3\%) konnten CPG 2 zugeordnet werden. In CPG 1 bzw. 3 befanden sich 18,2 \% bzw. 14,0\%. Die Veränderung zwischen den Messzeitpunkten war signifikant mit einem großen Effekt $(r=-0,55)$. Insgesamt verbesserten sich zwei Drittel $(67,5 \%)$ der Patienten in den CPG-Stufen, $43,9 \%$ der Patienten sogar um 2 oder mehr Stufen.
Diskussion: In dieser Studie konnte gezeigt werden, dass das Chronic Pain Grading auch im Kinder- und Jugendbereich eine gute Möglichkeit ist, um Therapie-Outcome zu erfassen und Veränderungen durch die Therapie abzubilden. Des Weiteren konnte repliziert werden, dass die interdisziplinäre Schmerztherapie eine wirksame Intervention für Jugendliche mit chronischen Schmerzen ist und sich die Schwere der Schmerzproblematik ein Jahr nach der Therapie deutlich reduziert.

\section{P12.03 - Know Body, Know Way, Know Action: Eine edukative Patientenschulung für Kinder und Jugendliche mit chronischen Schmerzen}

L. Höfel, U. Hammer, M. Storf, J.v. d. Beek, N. Draheim, A. Schramm, J. Haas, E. Schnöbel-Müller

Kinderklinik Garmisch-Partenkirchen gGmbH, Zentrum für Schmerztherapie junger Menschen, Garmisch-Partenkirchen, Deutschland

Fragestellung: Etablierung und Evaluation einer interdisziplinären und interaktiven edukativen Patientenschulung („Know How") für Kinder und Jugendliche mit chronischen Schmerzen.

Material und Methode: Auswahl von stationären PatientInnen (13-18 J.) im Zentrum für Schmerztherapie junger Menschen; Umsetzung einer regelmäßigen standardisierten Patientenschulung zur Vermittlung des bio-psycho-sozialen Schmerzverarbeitungsmodells; im Rahmen der stationären multimodalen Schmerztherapie erarbeiten ÄrztInnen, PsychologInnen und SozialpädagogInnen jeweils mit 8-10 PatientInnen über einen Zeitraum von vier Tagen edukative Inhalte zu chronischen Schmerzen. In dem interaktiven Schulungsprogramm („Know How“) werden sowohl medizinische Faktoren als auch psychologische Einflüsse basierend auf dem bio-psycho-sozialen Schmerzverarbeitungsmodell anschaulich und einprägsam dargestellt. Ziel ist, den PatientInnen Wissen über Entstehung und Aufrechterhaltung von akuten und chronischen Schmerzen zu vermitteln. Im Rahmen dieser Arbeit erfolgt eine erste Evaluation der Schulung bei 70 PatientInnen über einen Zeitraum von 6 Monaten.

Ergebnisse: Bisherige Rückmeldungen von 50 PatientInnen zeigen ein gutes Krankheitsverständnis und meist positive Bewertungen. Kritikpunkte wurden in die Konzeptentwicklung einbezogen und werden diskutiert.

Diskussion oder Schlussfolgerung: Voraussetzung für eine aktive Mitarbeit der PatientInnen und einen nachhaltigen Therapieerfolg in der multimodalen Schmerztherapie ist das Verständnis für die Erkrankung. Edukation im Gruppensetting unter Einbezug unterschiedlicher Fachrichtungen, vielfältiger didaktischer und interaktiver Vorgehensweisen ermöglicht einen intensiven Austausch und die Förderung von Akzeptanz und Selbstwirksamkeit hinsichtlich der Erkrankung. Die Studie zeigt, dass im Rahmen einer standardisierten Patientenschulung eine Verbesserung des Krankheitsverständnisses erreicht werden kann.

\section{P12.04 - Veränderung der Inanspruchnahme und Kosten nach einer intensiven pädiatrischen Schmerztherapie Ergebnisse einer GKV-Routinedatenanalyse}

\section{A. Ruhe, M. Frosch, J. Wager, B. Zernikow}

Vestische Kinder- und Jugendklinik- Universität Witten/Herdecke, Deutsches Kinderschmerzzentrum; Lehrstuhl für Kinderschmerztherapie, Datteln, Deutschland

Hintergrund: Chronische Schmerzen verursachen hohe ökonomische Kosten. Bereits im Kindes- und Jugendalter stellen chronische Schmerzen ein bedeutsames Gesundheitsproblem dar. Etwa 32\% der Schulkinder zwischen 8 und 16 Jahren berichten von wiederkehrenden Schmerzen, 5\% leiden unter schwer beeinträchtigenden chronischen Schmerzen. Diese Kinder konsultieren häufig verschiedene Ärzte oder andere Gesundheitsdienstleister. Ziel der Studie ist es, Veränderungen in der Inanspruchnahme und den Kosten ein Jahr vor und ein Jahr nach einer 3-wöchigen intensiven interdisziplinären Schmerztherapie für stark beeinträchtigte Kinder und Jugendliche zu untersuchen. 
Methodik: Die vorliegende Studie erfasst Kinder und Jugendliche, die zwischen November 2009 und Juli 2011 eine 3-wöchige stationäre interdisziplinäre Schmerztherapie am Deutschen Kinderschmerzzentrum durchlaufen haben. Von den 65 teilnehmenden Kindern liegen Routinedaten von sieben gesetzlichen Krankenversicherungen vor. Die Berechnung der jährlichen Kosten umfasst die ambulant ärztliche Versorgung, stationäre Behandlungen, Arzneimittelverordnungen sowie Heil- und Hilfsmittel. Es wurden Veränderungen in der Inanspruchnahme von Gesundheitsleistungen und Kosten im Jahr vor (PRÄ_1Jahr) und in dem Jahr nach einer intensiven interdisziplinären Schmerztherapie (POST_1Jahr) analysiert. Ergebnisse: Im ersten Jahr nach der intensiven interdisziplinären Schmerztherapie zeigte sich keine signifikante Reduktion der Gesamtkosten. Das Inanspruchnahmeverhalten veränderte sich hingegen deutlich. Signifikant mehr Kinder und Jugendliche haben eine ambulante Psychotherapie $(p=0,001)$ begonnen. Gleichzeitig nahm die Anzahl an Kindern, die stationär behandelt wurde vom Jahr vor der stationären Therapie (65\%) zu 1 Jahr nach Therapie (32\%) signifikant ab $(p=0,001)$. Es gab weniger stationäre Aufenthalte aufgrund einer primären chronischen Schmerzerkrankung in dem Jahr nach der Therapie $(p<0,001)$. Die Verordnung von Nicht-Opioiden reduzierte sich ebenfalls signifikant von PRÄ_1Jahr (71\%) zu POST_1Jahr $(42 \%)(p=0,001)$.

Diskussion: Die Ergebnisse zeigen, dass sich die Kosten bei Kindern und Jugendlichen mit einer schwer beeinträchtigenden chronischen Schmerzstörung im ersten Jahr nach einer intensiven Schmerztherapie nicht signifikant reduzieren. Allerdings scheint die Art der Inanspruchnahme zielgerichteter zu sein. Maßnahmen zur Differentialdiagnostik und nichtindizierte Interventionen nehmen ab. Dafür nehmen stärker indizierte Behandlungen wie eine ambulante Psychotherapie zu. Es bedarf zukünftiger Studien, die die ökonomischen Veränderungen nach einer intensiven interdisziplinären Schmerztherapie im Langzeitverlauf untersuchen.

\section{P12.05 - Der pädiatrische Schmerzpatient: Eine Basisdatenerhebung in der Kinder- und Jugendarztpraxis}

\section{K. Maaßen, M. Frosch, B. Zernikow, J. Wager \\ Vestische Kinder- und Jugendklinik - Universität Witten/Herdecke, Deutsches Kinderschmerzzentrum, Datteln, Deutschland}

Hintergrund: Wiederkehrende Schmerzen sind ein häufiges Symptom bei Kindern und Jugendlichen. Diese Schmerzen sind zumeist nicht auf eine definierte organische Erkrankung zurückzuführen. Viele Betroffene suchen jedoch einen Arzt auf. Bislang ist wenig über die Eigenschaften der Kinder und Jugendlichen bekannt, die mit Schmerzen in der pädiatrischen Primärversorgung vorstellig werden. Die aktuelle Studie befasst sich daher mit der Erfassung der Eigenschaften von Patienten, die sich aufgrund funktioneller Schmerzen in der Kinder-und Jugendarztpraxis vorstellen. Methodik: Insgesamt nehmen 21 Kinder- und Jugendarztpraxen aus Nordrheinwestfalen an der Studie teil. Sie rekrutierten bislang 125 Kinder- und Jugendliche zwischen 6 und 17 Jahren, die aufgrund funktioneller Schmerzen vorstellig wurden. Bei der Erstvorstellung werden demographische, schmerzbezogene und versorgungsbezogene Daten erhoben. Hierzu füllen Kinder und deren Eltern jeweils einen Fragebogen zu der aktuellen Schmerzproblematik des Kindes aus.

Ergebnisse: Bislang wurden 81 Mädchen (64,8\%) und 44 Jungen (35,2\%) im Alter von durchschnittlich 11,3 Jahren $(S D=2,9)$ in die Studie eingeschlossen. 26,3\% der rekrutierten Patienten haben einen Migrationshintergrund. Im Median leiden die vorstelligen Kinder seit 24 Wochen an Schmerzen. Über die Hälfte der Kinder leidet an Kopfschmerzen, etwa ein Drittel an Bauchschmerzen und lediglich 4,7\% geben Schmerzen im Rücken oder in den Extremitäten an. Die durchschnittliche Schmerzintensität (NRS 0 - 10) lag bei dreiviertel aller Befragten zwischen 4-8, der Mittelwert betrug 5,81 $(\mathrm{SD}=1,7)$. In den letzten 4 Wochen vor dem Erstkontakt nahmen 59,7\% aller Patienten Schmerzmedikamente ein. Knapp die Hälfte aller Schmerzpatienten hatte in den letzten vier Wochen schmerzbedingte Schulfehltage (Wertebereich $1-14 ; \mathrm{M}=3,3, \mathrm{SD}=2,3$ ). 26,2 \% gaben an, häufig oder immer beim Kontakt mit Freunden beeinträchtigt zu sein, $30,1 \%$ waren bei ihren Hausaufgaben häufig oder immer beeinträch- tigt und 22,7\% waren beim Ausführen ihrer Lieblingsbeschäftigung häufig oder immer eingeschränkt.

Schlussfolgerung: Kinder und Jugendliche, die mit funktionellen Schmerzen in der Primärversorgung vorstellig werden, berichten bereits eine hohe Schmerzintensität. Bei vielen zeigen sich zudem Einschränkungen der Funktionalität, die auf einen fortschreitenden Chronifizierungsprozess hindeuten. Informationen über die Charakteristika dieser Patienten geben erste Hinweise für die Entwicklung von patientenorientierten $\mathrm{Be}-$ handlungsansätzen in der Primärversorgung.

Förderung: Die finanzielle Förderung des Projektes erfolgt durch den Bund (Bundesministerium für Gesundheit).

\section{P12.06 - Mit Spaß lernen. Überprüfung eines Wissensfragebogens zur animierten Psychoedukation über chronische Schmerzen bei Schulkindern}

\section{F. Heuer', L. Stahlschmidt ${ }^{2}$, B. Zernikow', J. Wager ${ }^{2}$}

'Vestische Kinder- und Jugendklinik- Universität Witten/Herdecke, Deutsches Kinderschmerzzentrum; Lehrstuhl für Kinderschmerztherapie, Datteln, Deutschland, ${ }^{2}$ Vestische Kinder- und Jugendklinik Datteln, Deutsches Kinderschmerzzentrum, Datteln, Deutschland

Hintergrund: In Deutschland berichtet etwa die Hälfte der Kinder und Jugendlichen wiederkehrende Schmerzen in den letzten drei Monaten. Niedrigschwellige Interventionen können zu einem Zeitpunkt sinnvoll sein, an dem Kinder mit wiederkehrenden Schmerzen noch keine Schmerzstörung entwickelt haben. Eine wichtige Intervention kann hier die Edukation über Schmerzen sein. Basisinformationen zu chronischen Schmerzen im Kindes- und Jugendalter wurden in einem Animationsfilm umgesetzt („Den Schmerz verstehen und was zu tun ist ... in 10 Minuten“; https://www. youtube.com/watch? $\mathrm{v}=\mathrm{KpJfix} Y \mathrm{gBrw})$. Im Rahmen dieser Studie soll untersucht werden, ob durch das Schauen des Animationsfilms, das Wissen über Schmerzen zunimmt.

Methodik: Im Rahmen des Projektes wurde zunächst ein Multiple-ChoiceFragebogen mit 20 Fragen entwickelt (Schmerz-Wissen-Fragebogen), der das Wissen, das in dem Animationsfilm vermittelt wird, abfragt (Wertebereich: 0 bis 20). In einem kleinen Sample $(\mathrm{N}=18)$ chronischer Schmerzpatienten wurde die Verständlichkeit der Fragen und Antwortmöglichkeiten überprüft. Für die Studie wurden N=95 Schulkinder der Jahrgangsstufen 5-7 von zwei Gesamtschulen rekrutiert. Die Studie wurde in einem Vorher-Nachher-Design durchgeführt. Zum ersten Messzeitpunkt wurde der Schmerz-Wissen-Fragebogen von allen Schülern ausgefüllt. Zusätzlich machten sie Angaben zu ihren Schmerzerfahrungen der letzten drei Monate. Im Anschluss sahen die Schüler den Animationsfilm und beantworteten danach erneut den Schmerz-Wissen-Fragebogen (zweiter Messzeitpunkt).

Ergebnisse: Die Schüler sind zwischen 10 und 15 Jahren alt $(M=11,69$; $\mathrm{SD}=1,09)$. Die Geschlechterverteilung ist mit $45,3 \%(n=43)$ weiblicher und 54,7\% $(n=52)$ männlicher Probanden ausgeglichen. 95\% der Schüler gaben an, in den letzten drei Monaten Schmerzen gehabt zu haben. 32\% der Schüler hatten mindestens einmal pro Woche Schmerzen. Beim ersten Messzeitpunkt erreichten die Schüler einen durchschnittlichen Punktwert von 7,6 $(\mathrm{SD}=2,9)$ im Schmerz-Wissens-Fragebogen. Nachdem die Schüler den Film geschaut hatten, lag der durchschnittliche Punktwert bei 11,2 (SD =3,7). Insgesamt gibt es einen signifikanten Wissenszuwachs $(\mathrm{F}(1,93)=107,470 ; p<0,001)$. Mädchen und Jungen unterscheiden sich nicht bezüglich des Wissens $(\mathrm{F}(1,93)=3,861 ; p=0,052)$ und auch nicht bezüglich des Wissenszuwachses $(\mathrm{F}(1,93)=0,017 ; p=0,896)$. Schüler, die mindestens einmal pro Woche Schmerzen hatten, zeigen nach dem Schauen des Films einen signifikant stärkeren Wissenszuwachs als Schüler mit seltenen oder keinen Schmerzen $(\mathrm{F}(1,87)=5,992 ; p=0,016)$.

Schlussfolgerungen: Die Studie zeigt, dass der Film als sinnvolle Maßnahme genutzt werden kann, um Kinder über chronische Schmerzen aufzuklären. Kinder mit häufigen Schmerzen profitieren mehr von der Wissensvermittlung als Kinder, die mit der Problematik nicht so häufig konfrontiert werden. 
P12.07 - Schmerzreduktion beim Verbandswechsel - Projekt zur Vermittlung von Bewältigungsstrategien für Kinder mit Epidermolysis bullosa

B. Hübner-Möhler', A. Beissenhirtz' , K. Müller', B. Zernikow², B. Zernikow' 'Vestische Kinder- und Jugendklinik Datteln, Kinderpalliativzentrum, Datteln, Deutschland, ${ }^{2}$ Vestische Kinder- und Jugendklinik- Universität Witten/Herdecke, Deutsches Kinderschmerzzentrum; Lehrstuhl für Kinderschmerztherapie, Datteln, Deutschland

Hintergrund: Etwa zwei von 100.000 Kindern werden mit der seltenen genetisch bedingten Erkrankung Epidermolysis bullosa (EB) geboren. Durch eine mangelhafte Verankerung der Epidermis mit darunterliegenden Hautschichten kann bereits geringe Reibung zu Blasenbildung und schmerzhaften Wunden führen. Folgen sind u. a. Zusammenwachsen von Fingern und Zehen, Speisenröhrenverengung oder ein erhöhtes Hautkrebsrisiko; bei schweren Formen ist die Lebenserwartung verkürzt. Schmerzen erleben Kinder mit EB aufgrund ihrer Erkrankung, der Wundversorgung, therapeutischer und diagnostischer Maßnahmen, aber auch bei Alltagsaktivitäten und beim Spielen. Die meisten Schmerzen erleiden sie jedoch bei den täglich notwendigen Verbandswechseln, die lebenslang zum Familienalltag gehören. Schmerzbezogene Ängste und Stressempfinden können die Schmerzwahrnehmung erhöhen und die Durchführung des Verbandswechsels erschweren. Ein Projekt im Kinderpalliativzentrum Datteln vermittelt Eltern und Pflegenden Strategien, wie sie Kindern bei der Bewältigung der Schmerzen helfen können.

Ziele: des Projektes sind die Reduktion von Schmerzwahrnehmung, Angst und Stresserleben, um der Entwicklung einer chronischen Schmerzstörung vorzubeugen

Methoden: Bestandteile des Projektes sind: 1. Workshops für Eltern und Pflegende, 2. ein Informationsfilm und 3. Schriftliche Informationen für Eltern, Kinder und Pflegende. Informationen zu Schmerzen, Angst und Einflussfaktoren auf das Schmerzerleben bilden die Grundlage für das Verständnis, dass die Schmerzwahrnehmung beeinflussbar ist. Diese Erkenntnis wird genutzt, um negative Einflussfaktoren zu vermeiden und positive Einflussfaktoren gezielt anzuwenden, wie Entspannungsübungen, Ablenkung, positive Selbstinstruktion, aktive Mithilfe der Kinder, emotionale Unterstützung und Verstärkung. Der Informationsfilm zeigt Möglichkeiten und Grenzen der Umsetzung in einem Familienalltag.

Ergebnisse: Insgesamt 17 Teilnehmer (TN) wurden bisher in je einem Workshop (WS) für Pflegende (WS 1: $8 \mathrm{TN}$ ) und Eltern (WS 2: 9 TN) geschult. Die Auswertung erfolgte schriftlich, mündlich und in Form von Telefoninterviews. Die verschiedenen Bestandteile der Workshops wurden ausschließlich positiv bewertet (sehr zufrieden/überwiegend zufrieden). In drei Telefoninterviews zu Erfahrungen mit der Umsetzung der Tipps beschrieben Mütter insbesondere Ablenkungsstrategien und verändertes eigenes Verhalten als hilfreich. Sowohl Pflegende als auch Eltern empfahlen die Kooperation mit Erstversorgern und EB-Zentren, damit Strategien zur Schmerzreduktion bereits frühzeitig vermittelt werden können. Ausblick: Aufgrund der Rückmeldungen der WS-Teilnehmer wird das Projekt auch in EB-Zentren vorgestellt (Salzburg, Freiburg, Erlangen und Hannover). Mit Abschluss des Projektes im Frühjahr 2017 werden ein Informationsfilm sowie evidenzbasierte Lehrmaterialien (Manuale und Broschüren) zur Verfügung stehen, die als Grundlage für weitere Schulungen und Beratung von betroffenen Familien oder Pflegenden dienen können. Förderung: Der Beitrag wird unterstützt von Dr. Ausbüttel \& Co GmbH. 


\section{Autorenverzeichnis}

$\begin{array}{ll}\text { A } & \\ \text { Agar Meera } & \text { P11.04 } \\ \text { Attal Nadine } & \text { P08.08 } \\ \text { Auffenberg Eva S } & \text { Y10 } \\ \text { Augustin Miriam } & \text { P04.01 } \\ \begin{array}{l}\text { Azad Shahnaz } \\ \text { Christina }\end{array} & \text { SY31 }\end{array}$

\section{B}

Baron Ralf $\quad$ P08.08; P08.01; P09.07;

Bartels Max

Barth Felix

Béchir Markus

Becker Susanne

Beckmann Matthias

Beer Katrin-Grit

Beissenhirtz Andrea

Benson Sven

Berkemann Michelle

Berkemer Esther

Bernard Daniel

Berner Reinhard

Berth Hendrik

Besier Werner

Bettmann Jan

Beyer Marie-Lena

Bharat Chrianna

Bialas Patrick

Biet Cornelia

Binder Andreas

Bingel Ulrike

Binzen Uta

Birklein Frank

Blankenburg Markus

Boettger Karina

Böger Andreas

Böhm Julia

Böhme Julia

Bohne Tisha

Book Malte

Bösl Irmgard

Bouhassira Didier

Bressan Elisangela

Brinkschmidt Tamina

Brockmöller Jürgen

Bründel Karl-Heinz

Bundscherer Anika

Burdach Maximilian

C

$\begin{array}{ll}\text { Callan Michael } & \text { P08.05 } \\ \text { Carl Maria } & \text { P03.03 } \\ \begin{array}{l}\text { Casser Hans- } \\ \text { Raimund }\end{array} & \text { SY12; SY28; WS18 } \\ \text { Christiansen Sandra } & \text { SY30 } \\ \text { Claassen Hens } & \text { P03.09 } \\ \text { Clasen Svenja } & \text { P02.05 }\end{array}$

Cremer Alexander

Felix

Curt Armin

P04.02

P09.09

D

$\begin{array}{ll}\text { Damann Nils } & \text { P08.06 } \\ \text { de Witt Huberts } & \end{array}$

Jessie

Deichert Vasco P03.07; P03.06; SY04

Desch Simon

Diers Martin

Dietrich Johannes

Dietrich Sarah

Diezemann Anke

Dillinger Marion

Dobe Michael

Dohrenbusch Ralf

Doppler Kathrin

Draheim Nicole

P03.08

P02.04

P09.10

SY22

SY20; WS33

$\mathrm{P} 01.02$

P06.04

WS28

P09.08

$\mathrm{P} 12 ; \mathrm{SY} 02$

Drebenstedt Corinna P07.06

Dreher Kim

P09.11

Dresler Thomas

SY30; WS31

Dreyer Jan

P07.06

Dusch Martin

P11.01

Düsterhöft Jasmin

P09.07

E

Ebinger Friedrich $\quad$ SY02; SY07

Eich Wolfgang SY01;SY13

Eichelkraut Luise $\quad$ P03.03

Eitner Lynn $\quad \mathrm{P07} ; \mathrm{P03}$

Elsenbruch Sigrid $\quad$ P03.09; P10.04; SY37

Enax-Krumova Elena P03.05; P09.04; SY08; SY40

Enck Paul P10.04

Erkel Martin P09.06

Erlenwein Joachim SY04; SY11

Ernst Thomas $\quad$ P03.09

Esau Olga $\quad$ P08.01

Ettlin Dominik WS22

Evdokimov Dimitar P09.02

F

Faber Cornelius P04.05

Faymonville

Marie-Elisabeth $\quad$ SY17

Ferlemann Kerstin WS16

Filitz Jörg SY11

Finnern Marina $\quad$ SY21

Finnerup Nanna P08.08

Fischer Daniel $\quad$ P06.08

Fischer Marc P09.04

Fischer Joachim $\quad$ P11.01

Fischer Thomas $\quad \mathrm{P} 07.06 ; \mathrm{P} 11.04$

Flack Florentina $\quad$ P06.04; P12.01

Flamm Maria $\quad$ P11.06

Flor Herta $\quad$ P03.08

Foerster Alec P09.01

$\begin{array}{ll}\begin{array}{ll}\text { Förderreuther } \\ \text { Stefanie }\end{array} & \text { SY16; WS10 } \\ \text { Forkmann Katarina } & \text { P03.01 } \\ \text { Frank Johanna } & \text { P09.02 } \\ \text { Fraunberger Britta } & \text { P05.03 } \\ \text { Freilinger Tobias } & \text { SY10 } \\ \text { Frese Achim } & \text { WS12 } \\ \text { Freyhoff Jörg } & \text { SY39 } \\ \text { Freynhagen Rainer } & \text { P08.08 } \\ \text { Frosch Michael } & \text { P12.05;P12.04 }\end{array}$

G

Gabriel Holger P07.04

Gärtner Anne $\quad$ P10.03

Gaubitz Markus WS12

Gaul Charly SY25

Geber Christian WS21

Geis Francine P03.11; P10.07

Geiß Christa P06.03

Gergely Hedwig $\quad$ P02.05; P05.04

Gierthmühlen Janne P08.01; SY29; SY40

Ginzkey Christian SY23

Gnass Irmela $\quad$ P07.06; SY39

Göbel Anna $\quad$ P02.05; P05.04

Göbel Carl P02.05; P05.04

Göbel Hartmut $\quad$ P02.05; P05.04

Gockel Helmut P08.03

Göhmann

Heinz-Dieter P02.03

Goßrau Gudrun $\quad$ P02.01; P05.02

Graf Bernhard M. P02.06; P11.07

Graf Michael WS18

Greffrath Wolfgang P03.04; P04.08; P09.08

Grießinger Norbert $\quad$ P01.04; P06.03

Grömer Teja WS28

Große Katrin P11.02

Gruber Michael P02.06

Gruhl Elisa $\quad$ P05.02

$\mathrm{H}$

Haanpää Maija $\quad$ P08.08

Haas Johannes-Peter P12.03

Haase Ingo P06.02

Hacker Henriette $\quad$ P02.02; P03.02

Hägele Benjamin $\quad \mathrm{P} 05.06$

Hagenacker Tim P08.10; P09.05; P09.06

Hahnenkamp Klaus P02.02; P03.02

Hähner Antje P05.02

Hamberger Ulrich P07.02; SY06

Hammer Ulrike $\quad$ P12.03

Hampl Martin P09.01

Hansen Ernil SY17

Hansson Per P08.08

Hartwig Maite SY02

Hasenbring Monika SY01

Häuser Winfried $\quad$ SY17

Hebestreit Julia SY22

Hedrich Ulrike SY10

Hegelmaier Tobias P09.10 


\begin{tabular}{|c|c|c|c|c|c|}
\hline Heinen Florian & P05.01 & Koch Sandra & P06.06 & M & \\
\hline Heinze Axel & P02.05; P05.04 & Koenen Laura & & Maaßen Katharina & P12.05 \\
\hline Heinze-Kuhn Katja & P02.05; P05.04 & Ricarda & SY22 & Machelska Halina & P04.06 \\
\hline Hellriegel Jana & P08.01 & Kohler Jürgen & P11.05 & Magerl Walter & P03.04; P03.10; P03.11; \\
\hline Helmer Thomas & P06.02 & Kohnen Norbert & SY35 & & P10.07; SY05; SY 19 \\
\hline Helmers Ann-Kristin & P09.07 & Kolb Christiane & SY37 & Maier Christoph & SY20 \\
\hline Henrich Florian & P03.10; P03.11; P10.07 & König Simone & P08.06 & Maihöfner Christian & P08.04; P08.03; SY20; SY07; \\
\hline Hermann Christiane & SY36 & Kopf Andreas & SY31 & & SY19; SY34;WS21 \\
\hline Hermeling & & Kopke Kirsten & P07.06 & Mainka Tina & P08.09; P09.10 \\
\hline Lea Marleen & P03.07 & Korb Joachim & WS13 & Majeed Ronja & SY36 \\
\hline Herr Raphael & P11.01 & Körner Anne Juliane & P10.03 & Makarova Tanya & P04.04 \\
\hline Hess Andreas & P04.04; P04.03; P04.07 & Krafft Stefanie & P02.03 & Malik Rainer & SY10 \\
\hline Heuer Fabiola & P12.06 & Krallmann Claudia & P03.06 & Mallwitz Joachim & WS07 \\
\hline Higman Patience & P07.06 & Kramer Martin & P09.08 & Malsy Manuela & P11.07 \\
\hline Hofbauer Hannes & WS11 & Krämer Christopher & P01.05 & Mannil Lijo & P08.09 \\
\hline Höfel Lea & P12.03 & Krämer Heidrun & SY27 & Manthey Gesche & P09.07 \\
\hline Höffken Oliver & P03.05; P09.04; SY08; SY40 & Kreitz Silke & P04.03; P04.07 & Marschall Ursula & SY18 \\
\hline Hoheisel Ulrich & P04.09 & Krieg Sandro & P05.01 & Maslowski Sarah & P02.07 \\
\hline Holle-Lee Dagny & SY03;SY16 & Krisch Laura & P11.06 & Mattenklodt Peter & P06.03 \\
\hline Homann & & Kromer Thilo Oliver & P05.06 & Maurus Britta & WS07 \\
\hline Heinz-Herbert & P08.09 & Kröner-Herwig Birgit & P10.01 & Mayer Manfred & P11.01 \\
\hline Höper Johanna & WS32 & Kropp Peter & SY30;WS03 & Meents Jannis & P09.01 \\
\hline Hosie Annmarie & P11.04 & Kropp Peter & WSO3 & Mehdorn & \\
\hline Hostettler Pascal & P09.09 & Kuhnt Oliver & P06.02 & Hubertus Maximilian & P02.05 \\
\hline Hou Mei-Yin & WS22 & Külz Gabriele & P10.01 & Meier-Gerdhing Elisa & P01.05 \\
\hline Huber Julika & P08.09 & Kumowski Nina & P03.05; P09.10 & Meinecke Christoph & P02.05 \\
\hline Huber Michaela & P01.02 & Kurth Ingo & P09.01 & Meißner Winfried & SY07; SY24 \\
\hline Hubli Michele & P09.09 & Kuss Katrin & SY14 & Melsom Myhre & \\
\hline Hübner-Möhler & & Kutsche Lisa & P04.07 & Anette & P08.05 \\
\hline Bettina & P12.07 & Kvia Margrethe & P08.05 & Mense Siegfried & P04.09; SY13 \\
\hline Hüllemann Philipp & P09.07 & & & Meßlinger Karl & P05.03; SY23; SY32 \\
\hline Hupp Markus & P09.09 & & & Metje Eckart & P06.05 \\
\hline \multirow[t]{3}{*}{ Hüppe Michael } & P01.03; SY28 & $\mathrm{L}$ & & Meyer Bianca & SY30;WSO3 \\
\hline & & Labrenz Franziska & P03.09 & Meyer-Frießem & \\
\hline & & Labuz Dominika & P04.06 & Christine & P01.05 \\
\hline | & & Laekeman Marjan & P07.06; SY14;WS20 & Miltner & \\
\hline Icenhour Adriane & P03.09 & Lahmann Claas & WSO4 & Wolfgang H.R. & P03.03; P10.06 \\
\hline \multirow[t]{3}{*}{ Irnich Dominik } & WS27 & Lampert Angelika & P09.01 & Mittermaier Cornelia & P05.04 \\
\hline & & Landgraf Mirjam & P05.01 & Moll Julia & P06.08 \\
\hline & & Landmann Gunther & P08.07; P09.03 & Möller Nadin & P02.02; P03.02 \\
\hline J & & Lang Anne M. & WS06 & Moroder Anke & SY04 \\
\hline Jensen Troels S. & P08.08 & Langhorst Jost & P03.09 & Moser Maximilian & SY06 \\
\hline Junger Stefan & WS21;WS02 & Lassen Christoph & P01.02; P02.06 & Mothes-Lasch & \\
\hline Jürgens Tim & SY23; SY07; SY23 & Lassen Christoph & P02.06 & Martin & P07.05; P11.02; P11.03 \\
\hline Just Nathalie & P04.05 & Lautenbacher Stefan & P01.04 & Müller Katharina & P12.07 \\
\hline \multirow[t]{3}{*}{ Jutzeler Catherine } & P09.09 & Lautenschläger Eva & P05.02 & Müller Tom & P05.02 \\
\hline & & Leo Markus & P09.05; P08.10; P09.06 & Mund Kristin & P03.07 \\
\hline & & Leonhardt Corinna & P07.06 & Musial Frauke & P07.04 \\
\hline & & Liebl Wolfgang & P02.06 & Mußhoff Frank & P02.08 \\
\hline \multirow{2}{*}{ Kaiser Ulrike } & & Lienau Florian & P06.08 & & \\
\hline & $\begin{array}{l}\text { PU6.U;;PIO.03; PU6.06; } \\
\text { P06.09:P07.01:SY07:SY33 }\end{array}$ & Liesering-Latta Eva & WS26 & & \\
\hline Kaisler Miriam & P02.07 & Likar Rudolf & WS05 & $\mathrm{N}$ & \\
\hline Kaluza Luisa & P09.01 & Lind Alexander & P02.07 & Nagel Bernd & SY18 \\
\hline Kampik Daniel & P09.02 & Lindenberg Nicole & P01.02 & Namer Barbara & P09.01; P05.03 \\
\hline Kappesser Judith & SY33 & Lindig Martin & P01.03 & Nau Carla & P01.03 \\
\hline Kappis Bernd & WS30 & Lissek Vanessa & P12.01 & Neeb Lars & SY03; SY32 \\
\hline Kern Kai-Uwe & P08.03; WS25 & Liwowsky Iris & WS08 & Neoh Eng Chuan & P08.05 \\
\hline Kieseritzky Karin & SY35 & Locher Hermann & SY12 & Nestler Nadja & P11.06; SY39 \\
\hline Kirchner Juliane & SY22 & Löffler Annette & P02.04 & Neustadt Katrin & P07.01 \\
\hline Kitzrow Martin & P07.03 & Lucius Harald & WS17 & Niederberger Uwe & P02.05 \\
\hline Klan Timo & P10.02 & Luckett Tim & P11.04 & Niederberger Ellen & SY15 \\
\hline Klein Thomas & P03.10 & Lüdtke Kerstin & SY07; SY38;WS24;WS31 & Nielson Anneke & P05.04 \\
\hline Kliesch Sabine & $\begin{array}{l}103.10 \\
P 03.06\end{array}$ & Lukas Albert & P07.06 & Niemier Kay & WS14 \\
\hline Klimczyk Klaus & $\begin{array}{l}10.00 \\
10202\end{array}$ & Lukic Nenad & WS22 & Nilges Paul & SY14;WS09 \\
\hline Klinger Regine & SY30: SY22 & Lüscher Silvia & P01.06 & Nobis Hans-Günter & SY25;WS01 \\
\hline Klotzsche Marlen & $\begin{array}{l}\text { SY30; S 22 } \\
\text { P06.07; P10.03 }\end{array}$ & Lutz Johannes F. & P05.05; P07.05; P11.02; & & \\
\hline & & & P11.03 & & \\
\hline
\end{tabular}


Noll-Hussong

$\begin{array}{ll}\text { Michael } & \text { SY13 } \\ \text { Nothnagel Helen } & \text { P07.04 }\end{array}$

Nothnagel Helen P07.04

\section{0}

$\begin{array}{ll}\text { Oehler Beatrice } & \text { SY09 } \\ \text { Oehme Bastienne } & \text { P11.03 } \\ \text { Ohler C. } & \text { P09.08 } \\ \text { Orth Michael } & \text { P09.08 } \\ \text { Osterbrink Jürgen } & \text { P11.06 } \\ \text { Otto Bettina } & \text { P07.05; P11.02 } \\ \text { Özgül Özüm } & \text { P03.05; P09.04 }\end{array}$

P

$\begin{array}{ll}\text { Pannell Maria } & \text { P04.06 } \\ \text { Papenhoff Mike } & \text { WS29 } \\ \text { Pereira Manuel } & \text { P03.07; P03.06; SY40 } \\ \text { Petzke Frank } & \text { SY24 } \\ \text { Pfau Doreen } & \text { P03.10; P09.08 } \\ \text { Pfeifer Ann-Christin } & \text { P10.05 } \\ \text { Pfeuffer Stepanie } & \text { P01.01 } \\ \text { Pfingsten Michael } & \text { SY18 } \\ \text { Phillips Jane } & \text { P11.04 } \\ \text { Pielsticker Anke } & \text { WS23 }\end{array}$

Pogatzki-Zahn Esther P01.05; P03.07; P03.06; P04.02; P04.01; P04.05; SY15; SY24; SY26; SY04

Preißler Anke P07.01; P10.03

Puschmann

Anne-Katrin P06.01

Puta Christian P07.04; SY01

\section{R}

Rak Kristen SY23

Rasche Dirk P02.05

Rauschmann

Michael A.

Rehm Stefanie SY21; WS32

Reichl Sylvia P04.02

Reisig Florian WS05

Reuter Uwe SY16; SY32

Rice Andre P08.08

Richter Matthias $\quad$ P05.02; WS07

Rinert Janosch P09.09

Ristau Patrick P01.01

Rittner Heike SY09

Roderigo Till P10.04

Röhring Lena $\quad$ P03.10

Rolke Roman P09.11

Rosenberger Daniela P03.04; SY22

Rosner Jan P09.09

Rossi Maddalena P06.08

Rost Theresa $\quad \mathrm{P} 11.07$

Ruhe Ann-Kristin P12.04

Ruscheweyh Ruth P02.03; SY26

$\begin{array}{ll}\text { Rust B P06.07 } & \end{array}$

Rusu Adina Carmen SY36

\section{S}

Sabatowski Rainer $\quad$ P02.01; P05.02; P06.06; P06.09; P06.07; P07.01; $\mathrm{P} 10.03 ; \mathrm{SY} 18$

\begin{tabular}{|c|c|}
\hline $\begin{array}{l}\text { Sandkühler Jürgen } \\
\text { Schäfer Lukas }\end{array}$ & SY21 \\
\hline Christopher & P04.01 \\
\hline Schäfer Benjamin & SY38 \\
\hline Schaible Hans-Georg & SY12; SY27 \\
\hline Schankin Christoph & SY22 \\
\hline Scharnagel Rüdiger & P07.01 \\
\hline Scheel Jennifer & P01.04 \\
\hline Schenk Michael & SY35;WS11 \\
\hline Scheuren Paulina & P09.09 \\
\hline Schicht Linda & P02.01 \\
\hline Schilgen Markus & WS12 \\
\hline Schiller Margareta & P06.07 \\
\hline Schiltenwolf Marcus & P10.05 \\
\hline Schlereth Tanja & SY34 \\
\hline \multicolumn{2}{|l|}{ Schlickenrieder } \\
\hline Bastian & P04.08 \\
\hline Schlisio Barbara & P08.02 \\
\hline Schmelz Martin & SY34 \\
\hline Schmid Annina & P09.03 \\
\hline Schmidt Diana & P09.01 \\
\hline Schmidt Katharina & P03.01 \\
\hline Schmidt Jakob & SY22 \\
\hline Schmidt Manuela & SY09; SY15 \\
\hline \multicolumn{2}{|l|}{ Schmidt-Wilcke } \\
\hline Tobias & SY05 \\
\hline \multicolumn{2}{|l|}{ Schmitt } \\
\hline Linda-Isabell & P08.10; P9.05; P09.06 \\
\hline Schmitt Jochen & P07.01 \\
\hline Schneider Sabine & P08.03 \\
\hline \multicolumn{2}{|l|}{ Schnöbel-Müller } \\
\hline Elisabeth & P12.03 \\
\hline Schön Christoph & P06.03 \\
\hline \multicolumn{2}{|l|}{ Schönbach } \\
\hline Benjamin & P06.09; P10.03 \\
\hline Schramm Anja & P12.03 \\
\hline Schubert Maria & P03.06 \\
\hline Schuh-Hofer Sigrid & P03.04; P09.08 \\
\hline Schuler Matthias & P07.06;WS20 \\
\hline Schüler Markus & SY38 \\
\hline Schultes Bernd & SY06 \\
\hline Schulz Katja & P10.02 \\
\hline Schumann Frank & P05.02 \\
\hline Schwanebeck Uta & P02.01 \\
\hline Schwarze Christian & SY11 \\
\hline Schwarzer Andreas & P02.07 \\
\hline Schweinhardt Petra & P03.08 \\
\hline Seeger Dagmar & SY14 \\
\hline \multirow[t]{2}{*}{ Segelcke Daniel } & P04.02; P04.01; P04.05; \\
\hline & SY05; SY15 \\
\hline Segerdahl Märta & P08.08 \\
\hline Seip Jutta & P02.01 \\
\hline \multirow[t]{2}{*}{ Sens Elisabeth } & P05.05; P07.05; P11.02; \\
\hline & P11.03 \\
\hline Sergeeva Marina & P04.04 \\
\hline Serra Jordi & P08.08 \\
\hline Shaygan Maryam & P10.01 \\
\hline \multicolumn{2}{|l|}{ Siedentopf } \\
\hline Friederike & SY37 \\
\hline Siefke Hannelore & P05.05 \\
\hline Sindrup Sören & P08.08 \\
\hline Sinke Christopher & P03.01 \\
\hline Sirsch Erika & P07.06 \\
\hline Sisignano Marco & SY29 \\
\hline Sittl Reinhard & P01.04; P05.03 \\
\hline Skiera David & P05.02 \\
\hline Slater Helen & P08.05 \\
\hline
\end{tabular}

P03.06

P05.01

$\mathrm{P} 02.03$

P09.02; P09.08

SY03; SY07

$\mathrm{P} 06.05$

$\mathrm{P} 12.06 ; \mathrm{P} 12.02$

P01.06; P02.08; SY31

WS26

P02.08

WS22

P11.01

P08.07; P09.03

$\mathrm{P} 12.03$

P02.03; SY26

$P 04.03$

$\mathrm{P} 01.04 ; \mathrm{P} 05.03$

$\mathrm{P} 01.06 ; \mathrm{p} 02.08$

P09.07

P01.05

T

Tampin Brigitte $\quad$ P08.05; P09.03

Taubert Lisa $\quad$ P06.07

Temp Julia P04.06

Tesarz Jonas $\quad$ SY01; SY13

Theisinger Anna P05.02

Thiel Falk P01.04

Thomale Jürgen P09.06

Thöner Uta $\quad \mathrm{P} 11.03$

Timmann Dagmar P03.09

Tölle Thomas R. P08.08

Treede Rolf-Detlef $\quad$ P03.04; P03.10; P03.11;

$\mathrm{P} 04.09 ; \mathrm{P} 04.08 ; \mathrm{P} 08.08$; $\mathrm{P} 09.08 ; \mathrm{P} 10.07 ; \mathrm{P} 11.01$

Trepte-Freisleder

Florian

P05.01

P02.04

Trojan Jörg

$\begin{array}{ll}\text { Tronnier Volker } & \text { SY29 } \\ \text { Tzvetkov Mladen } & \text { P02.08 }\end{array}$

Ü

Üçeyler Nurcan P09.02; SY08

U

Uder Michael P04.03

Usichenko Taras I. P02.02; P03.02

V

v. d. Beek Jutta $\quad \mathrm{P} 12.03$

Vahldieck Carl P01.03

Vollert Jan $\quad$ P02.07; P03.05; P08.08;

P08.09; P09.10; P09.04

von der Brelie

Christian

P09.07

von der Hagen Maja P05.02

von Wachter Martin SY25; WS30

W

Wager Julia $\quad \mathrm{P} 06.04 ; \mathrm{P} 12.05 ; \mathrm{P} 12.06$; $\mathrm{P} 12.01 ; \mathrm{P} 12.04 ; \mathrm{P} 12.02 ; \mathrm{SY} 20$ 


\section{Autorenverzeichnis}

Wallburg Franziska

Walton Pat

Wank Isabel

P06.08

Wasner Gunnar

P07.07

Weiss Thomas

P04.07

WS21

Weißleder Jeannette P05.05

Wendschuh Kerstin P06.09

Werner Marc WS15

Wesselmann Ursula SY37

Wichmann Martina P03.06

Wiese Christoph

Wilhelm Ilca

P01.02; P02.06

Wilhelm Thomas

Winner Beate

Wippert Pia-Maria

Wirth Julia

P09.01

P06.01

P03.11; P10.07

Wirz Stefan

SY35; WS11

Wunder Stephanie

P07.03

Z

Zahn Peter

Zernikow Boris

P01.05

$\mathrm{P} 06.04 ; \mathrm{P} 12.05$;

$\mathrm{P} 12.06 ; \mathrm{P} 12.07 ; \mathrm{P} 12.01$;

P12.04; P12.02; SY20

Zhang Juanjuan P04.09

Zieglgänsberger

Walter

$\mathrm{P} 02.04$

Zimmer Uta

P05.02

Zitzmann Michael

P03.06 